EXPERIMENTAL INVESTIGATION OF CERTAIN INTERNAL CONDENSING AND BOILING FLOWS: THEIR SENSITIVITY TO PRESSURE FLUCTUATIONS AND HEAT TRANSFER ENHANCEMENTS

Michael Toomas Kivisalu

Recommended Citation

Kivisalu, Michael Toomas, "EXPERIMENTAL INVESTIGATION OF CERTAIN INTERNAL CONDENSING AND BOILING FLOWS: THEIR SENSITIVITY TO PRESSURE FLUCTUATIONS AND HEAT TRANSFER

ENHANCEMENTS", Dissertation, Michigan Technological University, 2015.

https://doi.org/10.37099/mtu.dc.etds/897 


\title{
EXPERIMENTAL INVESTIGATION OF CERTAIN INTERNAL CONDENSING AND BOILING FLOWS: THEIR SENSITIVITY TO PRESSURE FLUCTUATIONS AND HEAT TRANSFER ENHANCEMENTS
}

By

Michael Toomas Kivisalu

\begin{abstract}
A DISSERTATION
Submitted in partial fulfillment of the requirements for the degree of DOCTOR OF PHILOSOPHY
\end{abstract}

In Mechanical Engineering-Engineering Mechanics

MICHIGAN TECHNOLOGICAL UNIVERSITY

2015

(C) 2015 Michael T. Kivisalu 

This dissertation has been approved in partial fulfillment of the requirements for the Degree of DOCTOR OF PHILOSOPHY in Mechanical Engineering-Engineering Mechanics.

Department of Mechanical Engineering-

Engineering Mechanics

Dissertation Adviser: $\quad$ Dr. Amitabh Narain

Committee Member: Dr. J effrey Allen

Committee Member: $\quad$ Dr. Gordon Parker

Committee Member: $\quad$ Dr. Ranjit Pati

Committee Member: $\quad$ Dr. Yoke Khin Yap

Department Chair: $\quad$ Dr. William Predebon 



\section{DEDICATION}

\section{In memory of Mr. Paul Reichard.}

He, along with his brother, Kevin Reichard, and their parents, inspired me to study mechanical engineering through their support and encouragement of my mechanical projects while I was in high school. 



\section{Table of Contents}

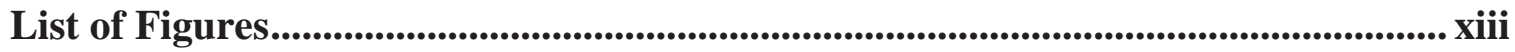

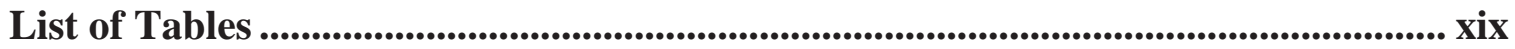

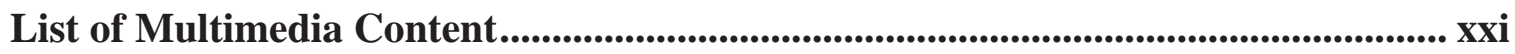

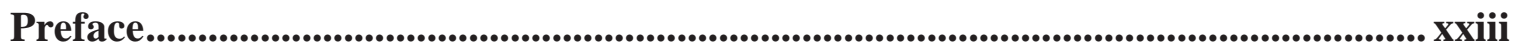

Acknowledgments ........................................................................................................................ xxxi

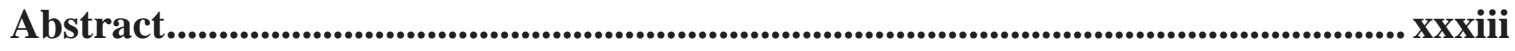

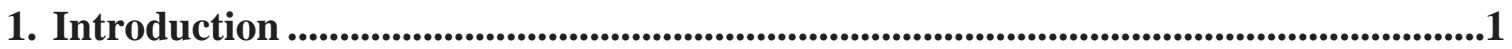

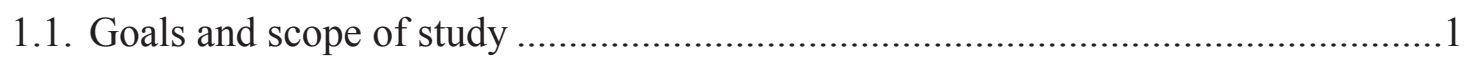

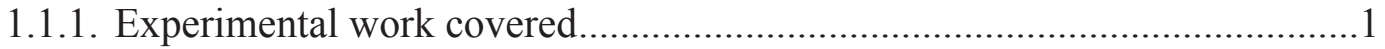

1.1.2. Revision of published material .........................................................

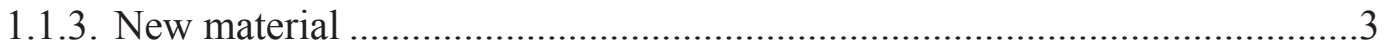

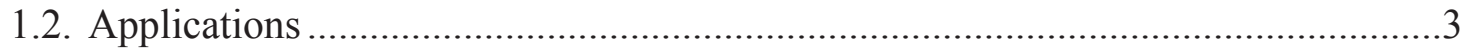

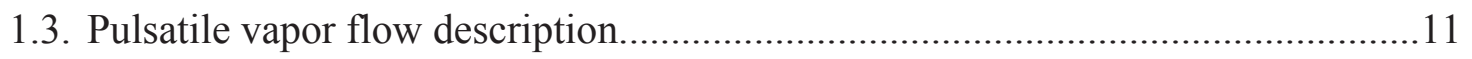

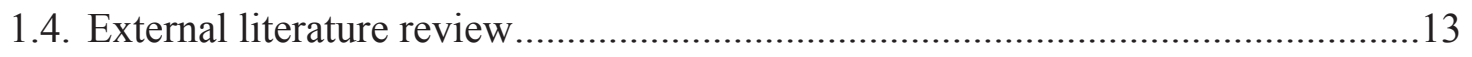

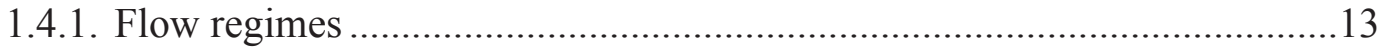

1.4.2. Instabilities, instability mechanisms, and critical heat flux......................13

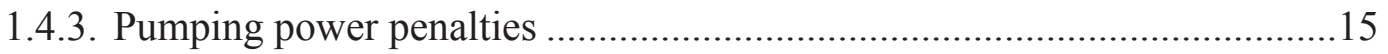

1.4.4. Differences between predominately gravity and shear driven boiling and

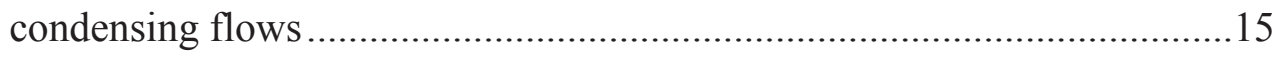

1.4.5. Enhancement approaches for pool and flow boiling — texturing of the heat-exchange surface (solid-liquid interface) and active utilization of enhanced heat-flux values at multiple contact-line-type thin film

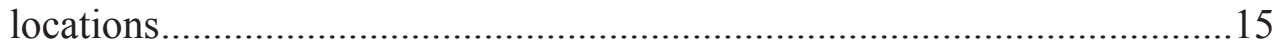

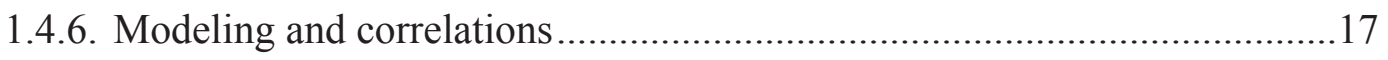


1.4.7. Phase-change pulsatile flow experiments................................................18

1.5. Research conducted at Michigan Technological University ................................20

1.5.1. Elliptic shear/pressure-driven internal condensing/boiling flow hypothesis

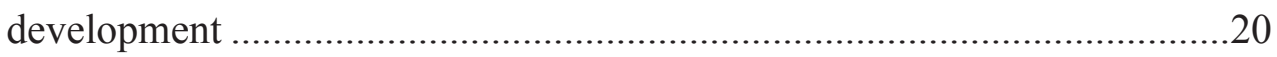

1.5.1.1. Early computational work ..............................................................20

1.5.1.2. Gravity-driven flows shown to be parabolic in nature....................22

1.5.1.3. Shear/pressure-driven unsteady, non-pulsatile flows found to be

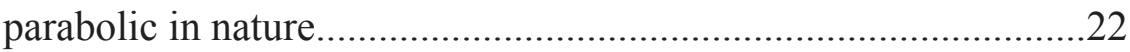

1.5.1.4. Elliptic sensitivity discovered in pulsatile shear/pressure-driven

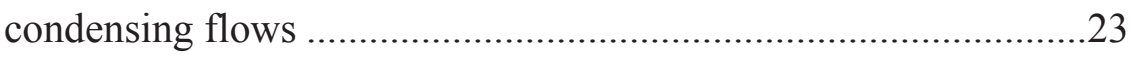

1.5.1.5. Current understanding on elliptic sensitivity in shear/pressuredriven pulsatile condensing and boiling flows................................24

1.5.2. Horizontal flow condenser performance ....................................................24

1.5.2.1. First attempts of horizontal condensing flow experiments at Michigan Technological University ...............................................24

1.5.2.2. Condensation experiments conducted in $2008-2010$...................25

1.5.2.3. Complete condensation experiments conducted in early $2011 \ldots . . .26$

1.5.2.4. Complete condensation experiments conducted in June 2011 ......26

1.5.2.5. Complete condensation experiments conducted in late 2011 ........27

1.5.2.6. All-annular condensation experiments conducted in

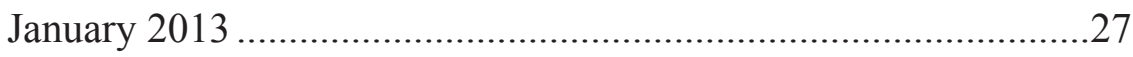

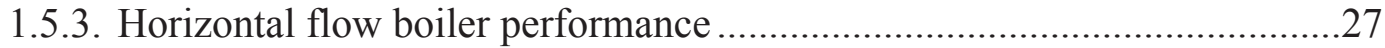

1.5.3.1. Flow boiling experiments conducted in 2012 ...............................27

1.5.3.2. Flow boiling experiments conducted in 2013 .................................28

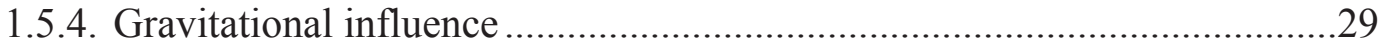

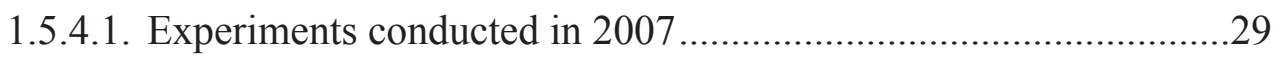

1.5.4.2. Changes in experimental hardware and procedures between 2007

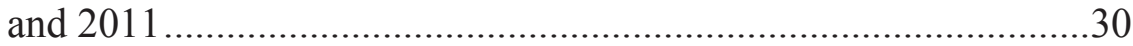

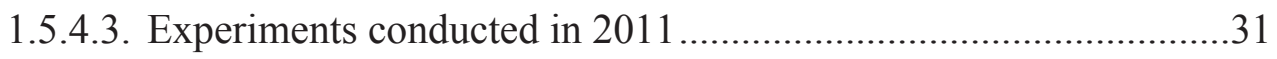

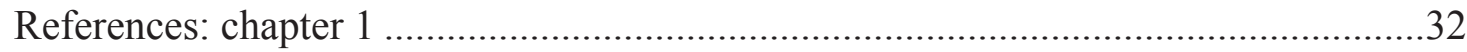




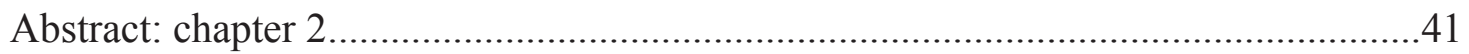

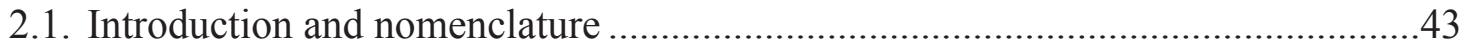

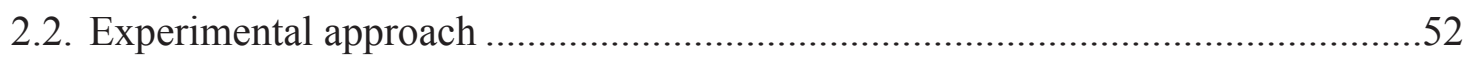

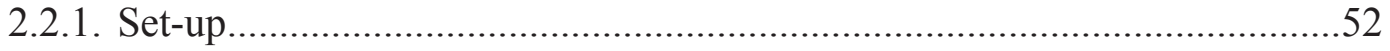

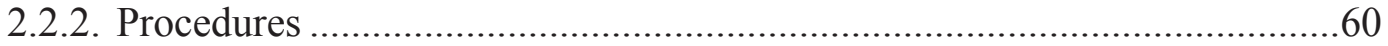

2.2.2.1. Innovative condensing flow experiments ................................63

2.2.1.1. Non-pulsatile flows resulting from no-imposed fluctuations (N-IF) cases ........................................64

2.2.2.1.2. Pulsatile flows resulting from imposed fluctuations (IF)

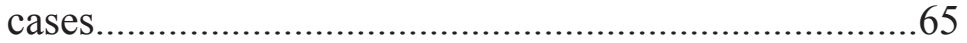

2.2.2.2. Innovative boiling flow experiments ......................................65

2.2.2.1. Non-pulsatile flows resulting from no-imposed fluctuations (N-IF) cases ........................................67

2.2.2.2. Pulsatile flows resulting from imposed fluctuations (IF)

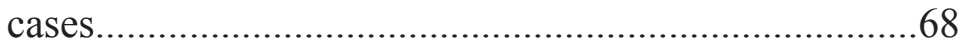

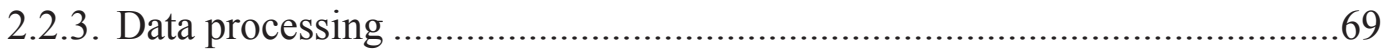

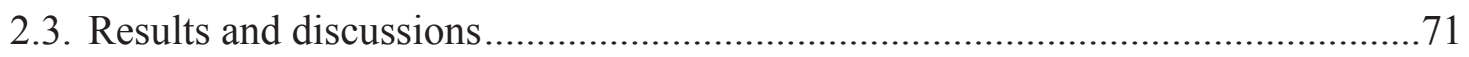

2.3.1. Results specific to condensing flows realized in the innovative arrangement

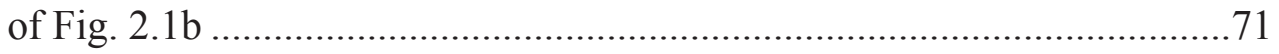

2.3.2. Discussion of results for condensing flows in the innovative arrangement of Fig. 2.1b .78

2.3.3. Results specific to boiling flows realized in the innovative arrangement of Fig. 2.2b

2.3.4. Discussion of results for boiling flows in the innovative arrangement of

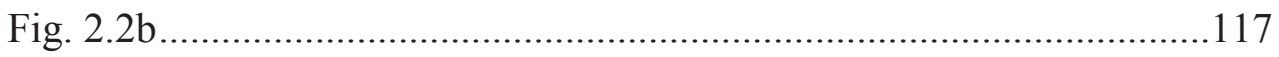

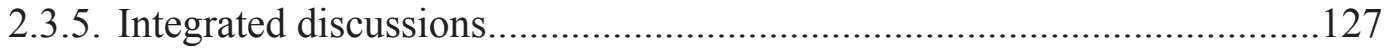

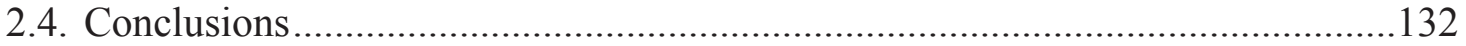

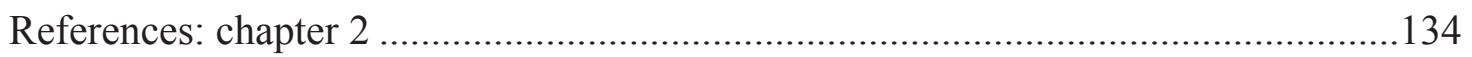


3. Completely condensing flow experiments........................................................................137

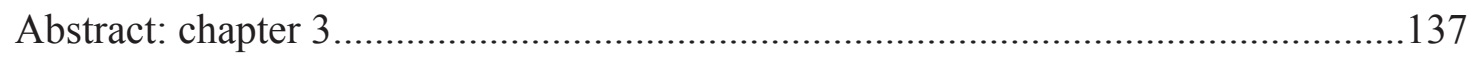

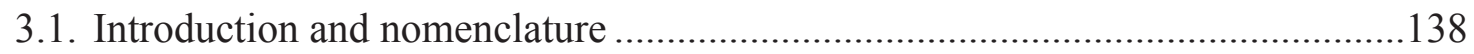

3.2. Terminologies used for the reported experimental investigation ........................143

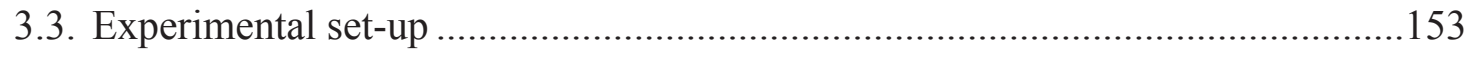

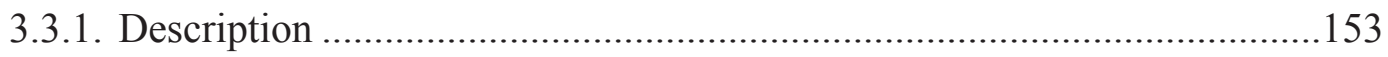

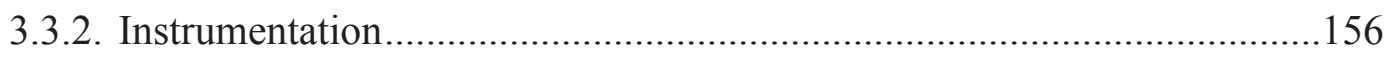

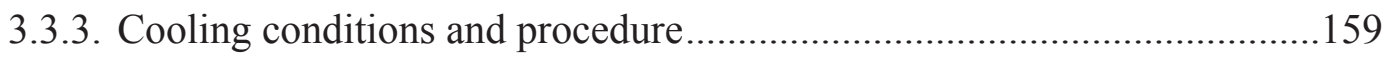

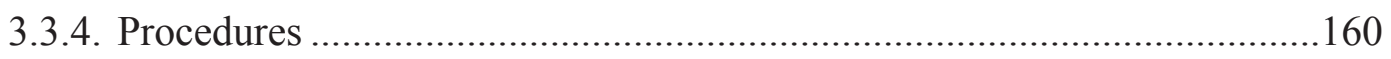

3.3.4.1. No-imposed fluctuation steady/quasi-steady flows ......................160

3.3.4.2. Quasi-steady response to imposed fluctuations .............................160

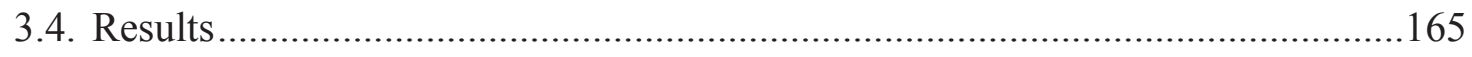

3.4.1. No-imposed fluctuation condensing flow results .......................................165

3.4.2. Quasi-steady condensing flows with imposed inlet-pressure

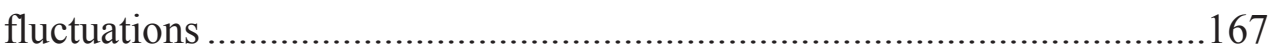

3.4.3. Experimental observation of heat-flux enhancement and changes to the flow due to imposed fluctuations ...........................................................177

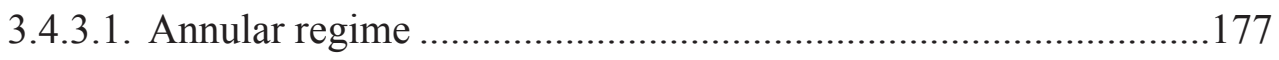

3.4.3.2. Non-annular regimes of fully condensing flows...........................184

3.4.3.3. All-annular regimes in innovative condensers employing partially

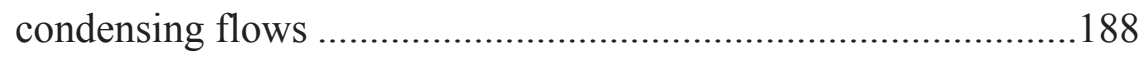

3.4.4. The role of amplitude-frequency content of fluctuations in attaining different quasi-steady flows ....................................................................189

3.4.5. Annular zone heat-flux enhancement mechanisms ....................................193

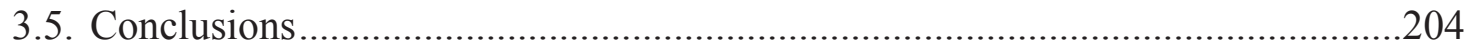

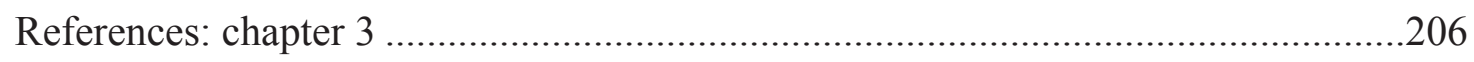

4. Summary and suggestions for future work.......................................................................211

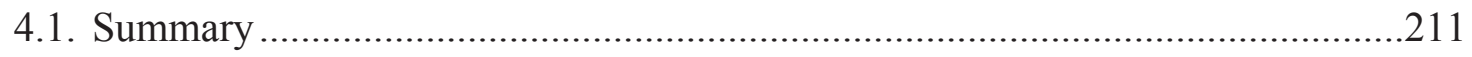

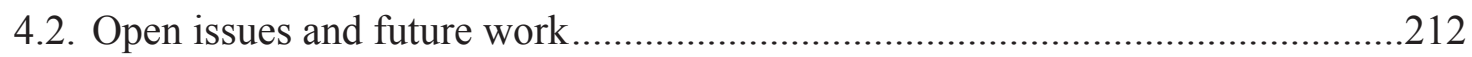


4.2.1. Opportunities for new experimental work.........................................212

4.2.2. Equipment issues affecting data reported here .....................................213

4.2.3. Relevant suggested, planned, and ongoing research .............................214

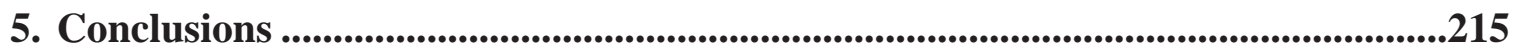

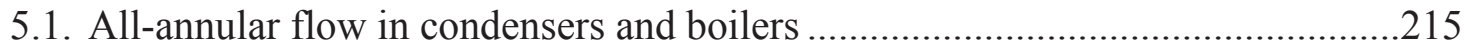

5.2. Pulsation-induced heat-flux enhancement ..................................................215

5.2.1. Heat-flux enhancement measured experimentally ................................216

5.2.2. Mechanisms behind observed heat-flux enhancement ............................216

5.2.3. Heat-flux enhancement limitations and recommendations .....................217 



\section{List of Figures}

Figure 1.1. Innovative vapor compression cycle for space-based applications. ...........7

Figure 1.2. Innovative Rankin power cycle for space-based applications. .................. 7

Figure 1.3. Non-dimensional flow regime schematic for two-phase flow. ...................10

Figure 1.4. Pulsator design used in the experiments. ........................................ 12

Figure 2.1. Side-view schematic and representative top-view photographs for (a) traditional and (b) innovative condenser operations.

Figure 2.2. Side-view schematic and representative top-view photographs for (a) traditional and (b) innovative boiler operations.

Figure 2.3. Schematics of the test-sections used in the innovative condensing and boiling flow experiments.

Figure 2.4. System schematic for flow condensation experiments relevant to the innovative design of Fig. 2.1b.

Figure 2.5. System schematic for flow boiling experiments relevant to the innovative design of Fig. 2.2b.

Figure 2.6. Mass flow rates and pressure-differences associated with runs C1, C4, and $\mathrm{C} 2$ in Tables $2.1-2.2$.

Figure 2.7. Absolute pressures and representative surface temperatures associated with runs $\mathrm{C} 1, \mathrm{C} 4$, and $\mathrm{C} 2$ in Tables $2.1-2.2$.

Figure 2.8. Heat-fluxes at $x=40 \mathrm{~cm}$ and representative temperature-differences associated with runs $\mathrm{C} 1, \mathrm{C} 4$, and $\mathrm{C} 2$ in Tables $2.1-2.2$.

Figure 2.9. Mass flow rates and pressure-differences associated with runs C5, C6, and $\mathrm{C} 10$ in Tables $2.1-2.2$.

Figure 2.10. Absolute pressures and representative surface temperatures associated with runs C5, C6, and $\mathrm{C} 10$ in Tables $2.1-2.2$.

Figure 2.11. Heat-fluxes at $x=40 \mathrm{~cm}$ and representative temperature-differences associated with runs C5, C6, and C10 in Tables 2.1 - 2.2.

Figure 2.12. Variation of time-averaged surface temperature with distance from the test-section inlet for innovative condensation experiments for which the imposed pulsation frequency was $10.6 \mathrm{~Hz}$. 
Figure 2.13. Variation of time-averaged surface temperature with distance from the test-section inlet for innovative condensation experiments for which the imposed pulsation frequency was around $28.4 \mathrm{~Hz}$.

Figure 2.14. Time-varying absolute pressures at $x=10 \mathrm{~cm}$ and $x=90 \mathrm{~cm}$ without imposed fluctuations (N-IF) and with imposed fluctuations of various amplitudes (amplitude increases with each case (run) from left to right) at $f_{P: V}=10.6 \mathrm{~Hz}$.

Figure 2.15. Time-varying absolute pressures at $x=10 \mathrm{~cm}$ and $x=90 \mathrm{~cm}$ without imposed fluctuations (N-IF) and with imposed fluctuations of various amplitudes (amplitude increases with each case (run) from left to right) at $f_{P: V} \approx 28.4 \mathrm{~Hz}$.

Figure 2.16. Time-varying pressure-differences over $0 \leq x \leq 40 \mathrm{~cm}$ and $0 \leq x \leq 90 \mathrm{~cm}$ without imposed fluctuations (N-IF) and with imposed fluctuations of various amplitudes (amplitude increases with each case (run) from left to right) at $f_{P: V}=10.6 \mathrm{~Hz}$.

Figure 2.17. Time-varying pressure-differences over $0 \leq x \leq 40 \mathrm{~cm}$ and $0 \leq x \leq 90 \mathrm{~cm}$ without imposed fluctuations (N-IF) and with imposed fluctuations of various amplitudes (amplitude increases with each case (run) from left to right) at $f_{P: V} \approx 28.4 \mathrm{~Hz}$.

Figure 2.18. Time-varying measured heat-flux and calculated film thickness at the $x=40 \mathrm{~cm}$ location in the test-section without imposed fluctuations (N-IF) and with imposed fluctuations of various amplitudes (amplitude increases with each case (run) from left to right) at $f_{P: V}=10.6 \mathrm{~Hz}$. 99

Figure 2.19. Time-varying measured heat-flux and calculated film thickness at the $x=40 \mathrm{~cm}$ location in the test-section without imposed fluctuations (N-IF) and with imposed fluctuations of various amplitudes (amplitude increases with each case (run) from left to right) at $f_{P: V} \approx 28.4 \mathrm{~Hz}$.

Figure 2.20. Fourier transform magnitude plots for $40 \mathrm{~cm}$ heat-flux and $0-40 \mathrm{~cm}$ pressure-difference in partial condensation. 102

Figure 2.21. Innovative condensing flow heat-flux enhancement chart with respect to amplitude of absolute pressure fluctuations at $x=10 \mathrm{~cm}$. 105 
Figure 2.22. Innovative condensing flow heat-flux enhancement chart relative to pressure-difference fluctuations over $0 \leq x \leq 40 \mathrm{~cm}$.

Figure 2.23. Innovative condensing flow heat-flux enhancement chart relative to absolute pressure fluctuations estimated at $x=40 \mathrm{~cm}$. 108

Figure 2.24. Variation of absolute pressure fluctuation amplitudes along the length of the condenser. 109

Figure 2.25. Inlet mass flow rate and pressure-difference data acquired at low DAQ rate for representative flow boiling cases.

Figure 2.26. Representative local surface temperature and local heat-flux data acquired at low DAQ rate for representative flow boiling cases at the $40 \mathrm{~cm}$ location.

Figure 2.27. Variation of time-averaged surface temperature with distance from the test-section inlet for representative flow boiling cases. 120

Figure 2.28. Time-varying pressure-difference $\Delta p_{0-40}$ without (solid line for case B1, along with dotted line indicating its average) and with (dashed line for case B2, along with light gray dashed line indicating its average) imposed inlet vapor pulsations, measured by the DPT-1 transducer between the $0 \mathrm{~cm}$ and $40 \mathrm{~cm}$ locations of Fig. 2.3b.

Figure 2.29. Time-varying heat-flux without (solid line for case B1, along with dotted line indicating its average) and with (dashed line for case B2, along with longer dashed line indicating its average) imposed inlet vapor pulsations, measured by a flush-type heat-flux meter (HFM-40 in Figs. 2.3b-c) at the $x=40 \mathrm{~cm}$ location.

Figure 2.30. Fourier transform magnitude plots for $40 \mathrm{~cm}$ heat-flux and $0-40 \mathrm{~cm}$ pressure-difference in flow boiling.

Figure 2.31. Time-varying film thickness (obtained from $\delta \equiv k \cdot\left(T_{s}-T_{\text {Sat }}\right) / q^{\prime \prime}$ under the theoretically verifiable assumption of conductive heat transfer mechanism across the film) at the $x=40 \mathrm{~cm}$ location, without (black, case B1) and with (gray, case B2) imposed inlet vapor pulsations. 
Figure 2.32. The experimentally obtained, primary dependence of time-averaged heat-flux values on the amplitude of inlet pressure fluctuations $\left(a_{p \mid 10}\right.$ in Table 2.3) for three different frequencies (approximately 2.4, 3.0, and 3.8 Hz as indicated in Table 2.3).

Figure 2.33. (a) Near-interface schematic of essential dynamics of the instantaneous spatial film thickness profile associated with micro-meter scale wavy film flows (over a wetting surface) encountered in pulsatile flow boiling (or flow condensation). (b) Time-varying film thickness profile at location $x=X^{*}$ in Fig. 2.33a.

Figure 3.1. Side views of (a) test-section and (b) instrumented condensing plate. .. 143

Figure 3.2. Schematic of the experimental flow loop used to investigate complete condensation.

Figure 3.3. The predicted/estimated ([41]) values of the time-varying mass flow rates $\dot{M}_{\text {OM-exit }}(t)$ at the orifice meter exit and $\dot{M}_{\text {in }}(t)$ at the test-section inlet. ....158

Figure 3.4. Sample flow visualization photographs for select cases in Table 3.1. ...163

Figure 3.5. Inlet mass flow rate and pressure measured at low DAQ rate for "passive" cooling condition cases NF3, P07, and P02 in Table 3.1. 166

Figure 3.6. Heat-flux (at $x=40 \mathrm{~cm}$ ) and exit pressure measured at low DAQ rate for "passive" cooling condition cases NF3 (N-IF), P07 (IF-LA), and P02 (IF-HA) in Table 3.1. 166

Figure 3.7. Quasi-steady wall temperature profiles under "passive" cooling conditions, with coolant (water) inlet temperature and flow rate maintained near $15^{\circ} \mathrm{C}$ and $7.9 \mathrm{lpm}$, respectively. 168

Figure 3.8. Non-dimensional wall temperature distribution chart under "passive" cooling conditions.

Figure 3.9. Quasi-steady wall temperature profiles under the "mixed" cooling conditions, with temperatures approximately held fixed at $38.5 \mathrm{~cm}$ and $58.5 \mathrm{~cm}$ downstream of the inlet.

Figure 3.10. Non-dimensional wall temperature distribution chart under the "mixed" cooling conditions. 
Figure 3.11. Dynamic data of the inlet vapor pressure $p_{i n}(t)$ for low amplitude (IF-LA), transition amplitude (IF-TA), and high amplitude (IF-HA) imposed pressure fluctuations at $10.1-10.2 \mathrm{~Hz}$ sampled for the "passive" cooling condition cases P07, P05, and P02, respectively, in Table 3.1. 173

Figure 3.12. Dynamic data of the pressure-drop $\Delta p_{40 \mathrm{~cm}}(t)$ for low amplitude (IF-LA), transition amplitude (IF-TA), and high amplitude (IF-HA) imposed pressure fluctuations at $10.1-10.2 \mathrm{~Hz}$ sampled for the "passive" cooling condition cases P07, P05, and P02, respectively, in Table 3.1.

Figure 3.13. Dynamic data of annular flow regime heat-flux $q_{40 \mathrm{~cm}}^{\prime \prime}(t)$ for low amplitude (IF-LA), transition amplitude (IF-TA), and high amplitude (IF-HA) imposed pressure fluctuations at $10.1-10.2 \mathrm{~Hz}$ sampled for the "passive" cooling condition cases P07, P05, and P02, respectively, in Table 3.1. 175

Figure 3.14. The amplitude-frequency data-matrix map for the imposed inlet pressure fluctuation cases in Table 3.1. 178

Figure 3.15. Heat-flux enhancement vs. $p_{\text {in }}$ amplitude chart for different pulsator frequencies.

Figure 3.16. Dynamic calculated data for film thickness (see Eq. 3.6-3.13) and pressure $p_{40 \mathrm{~cm}}(t) \cong p_{\text {in }}(t)-\Delta p_{40 \mathrm{~cm}}(t)$.

Figure 3.17. Heat-flux enhancement vs. $\Delta p_{40 \mathrm{~cm}}$ amplitude chart for different pulsator frequencies.

Figure 3.18. Approximate length of the annular flow regime for the cases in Table 3.1, organized by the imposed pulsation frequency $f_{P}$ and the cooling conditions, as the amplitude of imposed pressure-difference fluctuations increases.

Figure 3.19. Heat-flux removed from the region of the test-section where the flow is transitioning to non-annular flow regimes.

Figure 3.20. FFT magnitude plots of inlet pressure fluctuations $p_{i n}^{\prime}(t)$.

Figure 3.21. FFT magnitude plots of differential pressure across the first $40 \mathrm{~cm}$ of the test-section. 190

Figure 3.22. FFT magnitude plots of heat-flux at the $x=40 \mathrm{~cm}$ location in the test-section. 
Figure 3.23. FFT magnitude plots of estimated fluctuations in absolute pressure at the $x=40 \mathrm{~cm}$ location in the test-section. ...............................................192 


\section{List of Tables}

Table 2.1. Long-term (25 minutes) averages of selected flow variables from innovative flow condenser runs.

Table 2.2. Dynamic data averages (over average time-periods of $\overline{\Delta t}_{B}$ ) and amplitudes at frequency $f_{P: V}$ of selected flow variables from innovative condenser

runs.

Table 2.3. Selected flow variables from innovative flow boiler cases.

Table 3.1. Representative flow conditions for the cases used in the data-matrix of Fig. 3.14. 151

Table 3.2. Non-dimensional representation of cases in Table 3.1. 151 



\section{List of Multimedia Content}

Video 2-1. All-annular condensing flow, N-IF case C5 ...........................Video2-1.mov

Video 2-2. All-annular condensing flow, H-IF case C10 ..........................Video2-2.mov

Video 2-3. All-annular boiling flow, N-IF case B1 .................................Video2-3.mov

Video 2-4. All-annular boiling flow, H-IF case B2 ................................Video2-4.mov

Video 3-1. Completely condensing flow, N-IF.....................................Video3-1.mov

Video 3-2. Completely condensing flow, IF-HA ....................................Video3-2.mov

Video 3-3. Completely condensing flow, IF-HA (1/8 $8^{\text {th }}$ speed playback) ....Video3-3.mov 



\section{Preface}

\section{Chapter 1}

Figures $1.1-1.2$ were created by Michael T. Kivisalu with feedback provided by Prof. Amitabh Narain. The main design features in Figs. 1.1 - 1.2 were originally developed by M. Kivisalu. Figure 1.3 is taken from Nikhil Shankar's M.S. Thesis, and it is cited accordingly in the caption and text. Figure 1.4 was created by M. Kivisalu at the suggestion of Prof. A. Narain. It has been used as a back-up presentation slide by Prof. A. Narain for invited abstract IMECE 2014-40851 at the 2014 ASME International Mechanical Engineering Congress and Exposition, Montreal, Canada; it might also be included in future publications or conference presentations.

Sections 1.4.1 - 1.4.6 and the corresponding entries in the list of references for chapter 1 were adapted from material provided by Prof. Amitabh Narain at Michigan Technological University. Other than formatting changes, and updating the reference numbers, and minor content changes approved by Prof. A. Narain, these sections are identical to material provided by Prof. A. Narain. The remainder of this chapter is original, although some details of referenced works are mentioned or paraphrased where relevant to the discussion, particularly in section 1.5 .

\section{Chapter 2}

This chapter is a journal article which is reproduced with permission of the publisher, Elsevier. It has been reformatted, significantly modified from its published version, and expanded. All such reformatting, modifications, and expansion were performed by Michael.T. Kivisalu with minor editorial feedback from Prof. Amitabh Narain. These changes are not endorsed in any way by Elsevier or the International Journal of Heat and Mass Transfer.

\section{Published Document}

The order of authors was M.T. Kivisalu, P. Gorgitrattanagul, and A. Narain. Professor 
A. Narain developed the experimental objectives, and M. Kivisalu developed the experimental procedures in consultation with Prof. A. Narain. The experiments were supervised by M. Kivisalu. Patcharapol Gorgitrattanagul obtained most of the pictures and video for the experiments and assisted with the data collection. He also operated the flow loop when M. Kivisalu was absent from the laboratory.

Processing of the data was supervised by M. Kivisalu and accomplished by M. Kivisalu and P. Gorgitrattanagul. Data-dependent figures were created by M. Kivisalu at the direction of Prof. A. Narain. The tables were generated by M. Kivisalu. Schematics, particularly Figs. $2.1-2.5$ and 2.33, were created by P. Gorgitrattanagul at the direction of M. Kivisalu and Prof. A. Narain. The literature review and theoretical considerations, especially section 2.3.5, were written by Prof. A. Narain with minor feedback from M. Kivisalu, P. Gorgitrattanagul, and other graduate students. The conclusions were mainly developed by Prof. A. Narain and M. Kivisalu. Section 2.2 was written by M. Kivisalu with feedback from P. Gorgitrattanagul and Prof. A. Narain. Writing of section 2.3 was a collaborative effort of Prof. A. Narain and M. Kivisalu, with Prof. A. Narain providing more of the theoretical discussion and M. Kivisalu providing experimental details related to trends observed in the data-dependent figures.

\section{Expansion and Modification from Published Version}

Table 1 in the original document has been split into Tables $2.1-2.2$, and the order of cases has been revised to reflect a topical organization based on imposed pulsation pressure amplitude rather than the chronological order as in the original publication, but the original case names have been preserved.

Vapor velocity values were found to have been calculated incorrectly in the original publication. The corrected values were small, so they were removed from Table 1, and their ranges were reported in the text of section 2.3.1. A temperature-difference representative of just the first $40 \mathrm{~cm}$ of the test-section was added in Table 2.1, and the original $\Delta \mathrm{T}$ value from Table 1 was renamed $\Delta \mathrm{T}_{0-88.5}$, indicating the range of distances 
from the test-section inlet over which it is representative. The temperature of the fluid measured from the bottom at $x=0 \mathrm{~cm}$ was added to Table 2.1 as $T_{0}$. The value of $\Delta p_{10-90}$, measured from the difference between absolute pressures at $10 \mathrm{~cm}$ and $90 \mathrm{~cm}$ downstream of the test-section inlet, was also added to Table 2.1.

Table 2.2 contains new amplitude data taken from averaging multiple data files for each case. These values were taken from a single data file for each case in the original publication. Also, short-term averages from the dynamic data files considered for each case have been averaged together and reported in Table 2.2. The time-period of averaging has been reported as $\overline{\Delta t}_{B}$ in Table 2.2. Time-averaged calculated film thickness estimates, $\delta_{40}, 40 \mathrm{~cm}$ downstream of the test-section inlet, have also been added to Table 2.2. These values were not reported in the original publication.

Table 2 in the original publication has become Table 2.3 in this dissertation.

Figures $2.6-2.8$ have been added to allow comparison of the response of the low DAQ rate data in time at both frequency values investigated. Figures $2.9-2.11$ have been re-plotted using a lower cutoff frequency of digital filtering to further reduce noise and aliasing effects, and the data have been reorganized from the published version. Timevariations of absolute pressure measurements within the test-section, additional surface temperatures, and temperature-differences absent from the original publication have been included. Relevant discussion has been added to reflect these changes.

Figure 2.12 has been added to allow comparison of the condensing surface temperature distributions at both frequency values inverstigated. Figure 2.13 has been re-plotted with all of the cases of intermediate amplitude (only one N-IF case, one L-IF case, and one H-IF case were plotted in the original publication). Discussion on implications of condensing surface temperature variations with respect to heat-flux (and therefore heat-flux enhancement) variations along the length of the condenser has been added. 'Nodes' and 'antinodes' in surface temperature variation due to heat-flux enhancement 
along the length of the condenser have been noted in the captions of Figs. 2.12 - 2.13, consistent with expectations based on the hypothesized presence of approximately 'standing' acoustic waves in the vapor phase.

Figures 2.13, 2.15, and 2.17 have been added to allow comparison of representative timehistories of the dynamic data between both frequency values investigated. Figure 2.14 is new. Figures 2.16 and 2.18 have been re-plotted; the data have been organized differently from the original publication and new flow variables have been added. Specifically, the total test-section pressure-difference has been added to Fig. 2.16, and the calculated film thickness values have been included in the same graph with the measured heat-flux values. The time-histories of the N-IF and H-IF cases have been separated in Figs. $2.13-2.18$, whereas they were superposed in the original publication, and new cases have been added. The L-IF case C6 was added to Figs. 2.14, 2.16, and 2.18. Figures 2.13, 2.15, and 2.17 contain all four cases $(\mathrm{C} 1-\mathrm{C} 4)$ from Tables $2.1-2.2$. The discussions on these figures have been expanded accordingly.

Figure 2.21 has been modified from its original version to show the points from each of the individual dynamic data files superposed on the averaged response for each of the two frequencies. Figures $2.22-2.23$ have been added to show alternative means of characterizing pressure/flow pulsations within the test-section, and relevant discussion has been added. The pressure amplitude data in Fig. 2.23 has not been previously published anywhere. Figure 2.24 is completely new, and a discussion on the absorption of acoustic energy by the liquid/vapor interface has been added. The pressure amplitude data at $0 \mathrm{~cm}, 40 \mathrm{~cm}$, and $99.85 \mathrm{~cm}$ in Fig. 2.24 have not been previously published anywhere.

Significant discussion has been added related to the effectiveness of the thermoelectric modules (TECs of Figs. 2.3 - 2.5) in specifying the boiling surface temperatures in Fig. 2.27. The original published article referred that discussion to this dissertation since there was not room for it in the journal paper. It has not been published elsewhere. 
Figure 2.32 has been reformatted, and labels with relevant flow parameters have been added to make it easier to identify trends between the several data sets. More detail has been added to the caption of Fig. 2.32 .

Videos 2-1 - 2-4 have been added. These videos originally appeared in technical presentation HT2013-17477 at the ASME 2013 Summer Heat Transfer Conference, Minneapolis, Minnesota. Video processing was done by P. Gorgitrattanagul at the direction of M. Kivisalu with input from Prof. A. Narain.

\section{Chapter 3}

This chapter is a journal article which is reproduced with permission of the publisher, Elsevier. It has been reformatted, significantly modified from its published version, and expanded. All such reformatting, modifications, and expansion were performed by Michael.T. Kivisalu with minor editorial feedback from Prof. Amitabh Narain. These changes are not endorsed in any way by Elsevier or the International Journal of Heat and Mass Transfer.

\section{Published Document}

The order of authors was M.T. Kivisalu, P. Gorgitrattanagul, A. Narain, R. Naik, and M. Hasan. Professor A. Narain developed the experimental objectives, and M. Kivisalu developed the experimental procedures in consultation with Prof. A. Narain. The experiments were supervised by M. Kivisalu. Patcharapol Gorgitrattanagul obtained most of the pictures and video for the experiments and assisted with the data collection. $\mathrm{He}$ also operated the flow loop when M. Kivisalu was absent from the laboratory.

Processing of the data was supervised by M. Kivisalu and accomplished by M. Kivisalu and P. Gorgitrattanagul. Data-dependent figures were created by M. Kivisalu at the direction of Prof. A. Narain. The tables were generated by M. Kivisalu at the direction of Prof. A. Narain. Schematics, particularly Figs. $3.1-3.2$ and 3.4, were created by 
P. Gorgitrattanagul at the direction of M. Kivisalu and Prof. A. Narain. Figure 3.3 was created using data from CFD simulations performed by Ranjeeth. Naik on the orifice plate flowmeter of Fig. 3.2 following methods cited in section 3.3.1. R. Naik was also responsible for the comparison of the experimental results with strictly steady simulation results (section 3.5).

The introduction and terminology sections (sections 3.1 - 3.2) were mostly the work of Prof. A. Narain, with feedback from M. Kivisalu, P. Gorgitrattanagul, and R. Naik. Section 3.3 was written by M. Kivisalu with feedback from P. Gorgitrattanagul and Prof. A. Narain. Section 3.4 was a collaborative effort of Prof. A. Narain and M. Kivisalu, with Prof. A. Narain providing more of the theoretical discussion and most of the equations, and M. Kivisalu providing discussions of the experimental data and their trends observed in tables and data-dependent figures. The conclusions were mainly developed by Prof. A. Narain. Dr. Mohammed Hasan served as the monitor for the NASA grant under which these experiments, relevant simulations, and development of theory were conducted.

\section{Expansion and Modification from the Published Version}

Intermediate experimental cases were added to Tables 3.1 - 3.2. These were additional high amplitude imposed fluctuation cases which had inlet pressure amplitudes that were lower than those for the originally reported IF-HA cases but higher than those for the IF-TA cases. Additionally, the time-averaged exit pressure $\bar{p}_{\text {exit }}$ was added to Table 3.1 because its trends were discussed in the text. A column for an estimate of the total heat transfer rate within the test-section, $\overline{q_{\text {total }}^{\prime \prime}}$, was also added to Table 3.1. The column for $\mathrm{T}_{\text {sat }}$ was removed from Table 3.1 because its values showed very little variation for all of the cases; its value was reported in the caption for Table 3.1 instead.

Figure 3.3 was replaced with a version that also contained the dynamic mass flow rate at the orifice meter exit taken directly from the flow simulation, behind the curve for the dynamic mass flow rate estimated at the test-section inlet using the transient compressibility correction method of reference [41] in chapter 3. Figures $3.9-3.10$ 
have been added to allow comparison of the condensing surface temperature profiles between the "passive" and "mixed" cooling conditions, and they have not been reported elsewhere. Figure 3.11 is new, and it provides the dynamic response of the absolute pressure at the test-section inlet, which is not reported elsewhere for these experiments. Figures 3.12 - 3.13 have been re-plotted using data over the full time period considered in the representative dynamic data file analyzed for each case. This was done to allow low frequency absolute pressure variations in Fig. 3.11 to be properly represented, and to maintain consistency between Figs. 3.11 - 3.13 for comparison purposes.

A more detailed discussion on the significance of pressure-difference fluctuations within the flow condenser has been added to the text of section 3.4.3.1, and this has been used to introduce the alternative heat-flux enhancement chart of Fig. 3.17. Figure 3.17 is new, and it is not reported elsewhere. A new discussion of the mechanical energy supplied to the condensing flow by the combination of pressure and flow pulsations within the annular zone follows.

A new discussion on the effect of the pulsations on the length of the annular flow regime within the condenser has been added in section 3.4.3.2. Visual length estimates of the annular flow regime for the cases in Table 3.1 have been provided in Fig. 3.18. Figure 3.18 is new, and it has not been published elsewhere. The non-annular zone heat load variations are presented in Fig. 3.19, which is new and has not been reported elsewhere. Large differences between the trends, for the two cooling conditions, of indirect heat-flux estimates near the transition to non-annular flow regimes, reported in Fig. 3.19, are discussed.

The FFT magnitude plot of inlet absolute pressure fluctuations has been added as Fig. 3.20. This figure is important for showing that the harmonics of the frequency of imposed fluctuations at the inlet of the condenser are not significant, and it has not been reported elsewhere. The FFT magnitude plot of absolute pressure calculated at the $40 \mathrm{~cm}$ location within the condenser is added as Fig. 3.23. This figure is mentioned in 
the original text, but omitted from the original published paper for brevity, and it has not been reported elsewhere.

New discussion on the interfacial phenomena behind the realization of the observed heat-flux enhancement has been added in section 3.4.5, and details have been referred to chapter 2. A potentially limiting factor on the maximum heat-flux enhancement that can be realized, based on the nature of this caustive factor, has been added at the end of section 3.4.5.

Videos 3-1 - 3-3 have been added. These videos originally appeared in technical presentation IMECE2011-63281 at the ASME 2011 International Mechanical Engineering Congress and Exposition, Denver, Colorado. Video processing was done by P. Gorgitrattanagul at the direction of M. Kivisalu with input from A. Narain.

\section{Chapter 4}

This chapter combines hand-written notes from Prof. Amitabh Narain with original work by Michael Kivisalu. The level of contribution was approximately evenly divided.

\section{Chapter 5}

This chapter consists of original work by Michael Kivisalu. 


\section{Acknowledgments}

I gratefully acknowledge the support and enouragement of my adviser, Prof. Amitabh Narain, without which this document, along with my other publications, would not have been possible. His patience and understanding during challenging times for the research and for me personally have gone far beyond the call of duty, and his genuine concern for me professionally and personally are greatly appreciated.

Also, my parents have stood by me in this work and supported me in all the stuggles and adjustments that I have had to make in the process of this research and in my work that preceded it. They have given me valuable advice when needed, and othewise have provided me with listening ears and words of encouragement. Without them this work would not have been possible. I love them and am deeply grateful for their support.

My brother Stephen, other family members, people from my church, and friends have been very supportive, have provided advice and accountability, and have prayed with much perseverance for my work on this project. Their support has been indispensible. Many faculty, staff members, and current and former students at Michigan Technological University have gone out of their way to encourage me in this endeavor, and for that I am deeply thankful.

Lastly, although by no means least in importance, I acknowledge our creator, God, who is the reason for my existence and who fashioned my mind and body so that I would be able to learn and understand this world; a small part of that learning is reflected in my part of the work in this document. Through the forgiveness and life of Jesus Christ, my life has purpose and meaning; and through the power of God's Holy Spirit, my life has been sustained this far. It is only by God's grace that I am alive now and have been able to participate in the work embodied in this document. Many specific prayers related to this work have been answered, allowing me to make discoveries and advance past challenges that would otherwise have prevented me from continuing in this research. Words cannot express my gratitude. 



\section{Abstract}

Space-based (satellite, scientific probe, space station, etc.) and millimeter - to - microscale (such as are used in high power electronics cooling, weapons cooling in aircraft, etc.) condensers and boilers are shear/pressure driven. They are of increasing interest to system engineers for thermal management because flow boilers and flow condensers offer both high fluid flow-rate-specific heat transfer capacity and very low thermal resistance between the fluid and the heat exchange surface, so large amounts of heat may be removed using reasonably-sized devices without the need for excessive temperature differences. However, flow stability issues and degredation of performance of shear/ pressure driven condensers and boilers due to non-desireable flow morphology over large portions of their lengths have mostly prevented their use in these applications. This research is part of an ongoing investigation seeking to close the gap between science and engineering by analyzing two key innovations which could help address these problems.

First, it is recommended that the condenser and boiler be operated in an innovative flow configuration which provides a non-participating core vapor stream to stabilize the annular flow regime throughout the device length, accomplished in an energy-efficient manner by means of ducted vapor re-circulation. This is demonstrated experimentally.

Second, suitable pulsations applied to the vapor entering the condenser or boiler (from the re-circulating vapor stream) greatly reduce the thermal resistance of the already effective annular flow regime. For experiments reported here, application of pulsations increased time-averaged heat-flux up to $900 \%$ at a location within the flow condenser and up to $\mathbf{2 0 0} \%$ at a location within the flow boiler, measured at the heat-exchange surface. Traditional fully condensing flows, reported here for comparison purposes, show similar heat-flux enhancements due to imposed pulsations over a range of frequencies.

Shear/pressure driven condensing and boiling flow experiments are carried out in horizontal mm-scale channels with heat exchange through the bottom surface. The sides and top of the flow channel are insulated. The fluid is FC-72 from 3M Corporation. 



\section{I ntroduction}

This work comprises two published journal articles which have been significantly revised and updated. Before these articles is an introductory chapter (this chapter), giving a general context for the work. After the articles is a chapter (chapter 4) devoted to summarizing the results from the articles and presenting open research issues related to the content of the articles. Ongoing, planned, and suggested research activities for Prof. Amitabh Narain's research group in continuation of the research presented here are also briefly mentioned. The last chapter (chapter 5) brings together the earlier material in a succinct list of conclusions.

\subsection{Goals and scope of study}

There are three main goals of this dissertation:

1. Report relevant experimental data collected from June 2011 through January 2013 as a summary of the observed experimental sensitivities of internal shear-driven condensing and boiling flows to inlet pressure fluctuations (or pulsatile flows). Report associated observed heat-flux enhancement phenomenon.

2. Update and combine previous publications by the author (which used these data sets) to provide a basis for comparison and further analysis that is not possible within the context of the original publications.

3. Extend the analysis from these relevant publications to reflect more recent discovery and provide additional understanding and insight. Report relevant detail that was previously omitted for brevity.

\subsubsection{Experimental work covered}

The experimental data reported here were collected on June 15, 2011; June 18 - 19, 2011; January 30 - 31, 2013; February 17, 2013; and June 15 - 16, 2013. They cover the response of 3 classes of internal phase-change flows to imposed upstream pressure pulsations at various dominant amplitudes and frequencies: complete flow condensation, annular flow condensation, and annular flow boiling with dry-out near the boiler exit. 
Complete shear-driven flow condensation, in which a pure vapor flows into the condenser and pure liquid flows out of the condenser, was investigated in the June 15-16 and June $18-19,2011$ experiments. Annular shear-driven flow condensation, a subset of incomplete flow condensation in which the annular flow regime covers the entire length of the flow condenser and its inlet flow is pure vapor, was investigated in the January $30-31,2013$ experiments. Annular shear/pressure-driven flow boiling, in which liquid and vapor were separately injected at the flow boiler inlet and all the liquid was boiled off within the length of the flow boiler - yielding predominately annular flow throughout its length — was investigated in the February 17 and June 15 - 16, 2013 experiments. Only relevant subsets of these data are reported here. These subsets were chosen from the available experimental data to show the response of the flows to various amplitudes of inlet vapor pressure pulsations at two or more frequencies for each class of internal, shear-driven phase-change flow. Particular attention is given to those conditions which led to the highest observed enhancements in time-averaged local heat-flux at the measurement location.

The data reported here represent examples of these flow classes; they are not comprehensive for the working fluid and experimental configuration, and they do not represent the total of similar data acquired from the same experimental flow loop and its modifications. They were chosen because they clearly demonstrate the observed heat-flux enhancement phenomenon and enable a preliminary analysis of its potential causes and applications. Since the annular flow conditions are more desirable in practical applications, especially for shear/pressure-driven flow boiling, as described in section 1.2, this dissertation does not cover any flow boiling experiments in which only pure liquid enters the boiler (see section 1.5.3.1).

\subsubsection{Revision of published material}

The material published in [1] has been updated and included as chapter 2, providing an overview of the author's research on innovative partially condensing and boiling shear-driven flows and their sensitivity to pressure/flow fluctuations. The material 
published in [2] has been updated and included as chapter 3, providing an overview of the author's research on completely condensing flows and their sensitivity to pressure/flow fluctuations. Chapter 4 summarizes the material in chapters $2-3$, and it explains some open research issues with suggestions for future work. Chapter 5 provides a list of conclusions relevant to this entire dissertation.

\subsubsection{New Material}

New figures and tables augment those appearing in the original publications, allowing for a more thorough analysis. A specific description of what is meant by "pulsatile flow" is provided in section 1.3. Relevant details on the experimental system and methods are added to chapters $2-3$, which were not published previously for sake of brevity.

Some of the reported data in chapter 2 are replaced with data processed using more comprehensive methods. Table 2.2 reports statistically averaged values of relevant measured and calculated pulsatile flow variable amplitudes for the annular flow condensation cases. These flow variable amplitudes were previously published using only one representative dynamic data file for each case (set of operating parameters). Chapter 4 is entirely new material, athough some of its summary refers to previously published material within chapters $2-3$. Conclusions in chapter 5 have been re-written from similar published material to reflect a more holistic analysis based on the revised versions of chapters $2-3$.

\subsection{Applications}

Flow condensers and flow boilers may be found in many common devices, such as refrigerators, air conditioners, and power plants. These gravity-influenced millimeter - macro-scale applications are well-established, and in most cases thermal resistances outside the phase-change flow channel(s) or tube(s) dominate heat transfer, greatly reducing the usefulness of improvements based on the research reported here. Nevertheless, exceptions may exist, as refinement in the design of compact heat 
exchangers continues to reduce thermal resistances within and around these devices.

However, shear/pressure-driven (gravitational forces small compared to other forces acting on the fluid) flow condensers and flow boilers are being considered for future thermal management in space-based devices such as satellites, space stations, and scientific probes to provide cooling or heating to onboard equipment. They are also being considered for cooling of high powered electronics and waste heat recovery. In these applications, they are being considered due to the high heat transfer coefficients which may theoretically be attained with phase-change flow. However, they have not yet been effectively realized in these applications due to specific challenges which the research reported here seeks to address.

These challenges may be summarized as:

(i) difficulty managing the distribution of phases within the condenser or boiler especially for space applications, where gravitational forces cannot be used to maintain phase separation,

(ii) difficulty maintaining steady flow within the condenser or boiler - condensing and boiling flows which rely on pressure drop to move the liquid and vapor phases within the device tend to experience large flow transients and instabilities,

(iii) problems with degraded heat transfer and high thermal resistance for certain non-annular arrangements of the liquid and vapor phases, and

(iv) for certain condenser or boiler geometries and working fluids, unacceptably high pressure drops associated with the non-annular flow regimes.

Annular flow is defined by a pure vapor flow in the interior of the flow channel, with the liquid phase forming a thin film on one or more inside surfaces of the flow channel. Annular films may be wavy, but they are no longer considered to be annular if the waves break or curve such that there are multiple liquid-vapor interfaces between the inside surface on which the film is flowing and the center of the flow channel at a particular cross-section of the flow. 
The experiments reported here address two important issues related to these challenges, namely the control of the liquid-vapor flow morphology (shape of the liquid-vapor interface) within a shear/pressure-driven condenser or boiler (to a desirable stable annular configuration), and the enhancement of otherwise low heat transfer coefficients in condensers and boilers in which liquid flow must be driven primarily by the adjoining vapor flow through the shear stress that it provides to the liquid at their interface. This vapor flow is driven by the pressure-drop across the device; however, the local vapor flow rates within the device (driven by pressure variations along the length of the device) become very low in low-vapor-quality regions of the device, leading to eventual destabilization of the annular flow regime (see chapters $2-3$ ).

These shear-driven internal condensing and boiling flows are of interest because they occur in horizontal, micro-gravity, and micro-meter scale hydraulic diameter ducts. As previously mentioned, such ducts have applications in next generation space-based and military aircraft thermal management systems, as well as in high power electronic cooling applications [3 - 4]. On the component level, these flows are of interest in allowing stable operation of condensers and boilers in space-based thermal management systems.

The heat-flux enhancement phenomenon reported here may be utilized to help to significantly reduce the required size of condensers and boilers in these space-based thermal management systems, while at the same time improving component reliability and predictability of performance. This is necessary to allow the thermally efficient phase-change cycles to be utilized for space-based thermal management. This application provided the impetus for NASA funding through grants NNC04GB52G and NNX10AJ59G, which provided a significant portion of the funding for the research reported here, the balance being funded through NSF grant NSF-CBET-1033591.

System design concepts were prepared for further technology development work proposed to NASA as a result of the work reported here. These system concepts, which 
were prepared for discussions with personnel at the NASA Glenn Research Center in September, 2011, are schematically represented here in Figs. 1.1 - 1.2 on the next page, which have been adapted from their original form for clarity. For some thermal management systems which do not require a vapor compression cycle, the configuration of Fig. 1.2 may be used without the turbine or generator. 


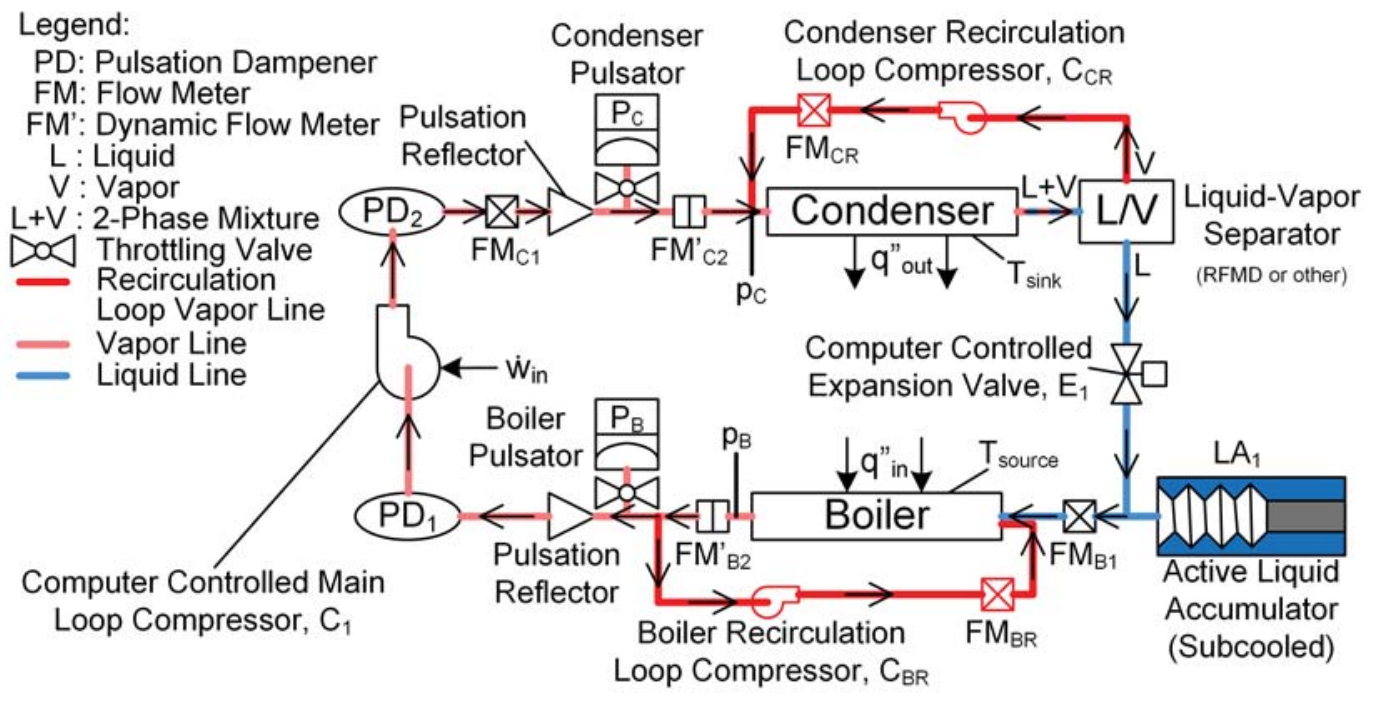

Figure 1.1. Innovative vapor compression cycle for space-based applications. For this cycle, the condenser pressure, $p_{C}$, is greater than the boiler pressure, $p_{B}$, such that heat rejection temperature, $T_{\text {sink }}$, is greater than heat absorption temperature, $T_{\text {source }}$. The work input to the compressor, $\dot{w}_{i n}$, drives the fluid flow, supplying the energy necessary to move heat from the lower $T_{\text {source }}$ to the higher $T_{\text {sink }}$.

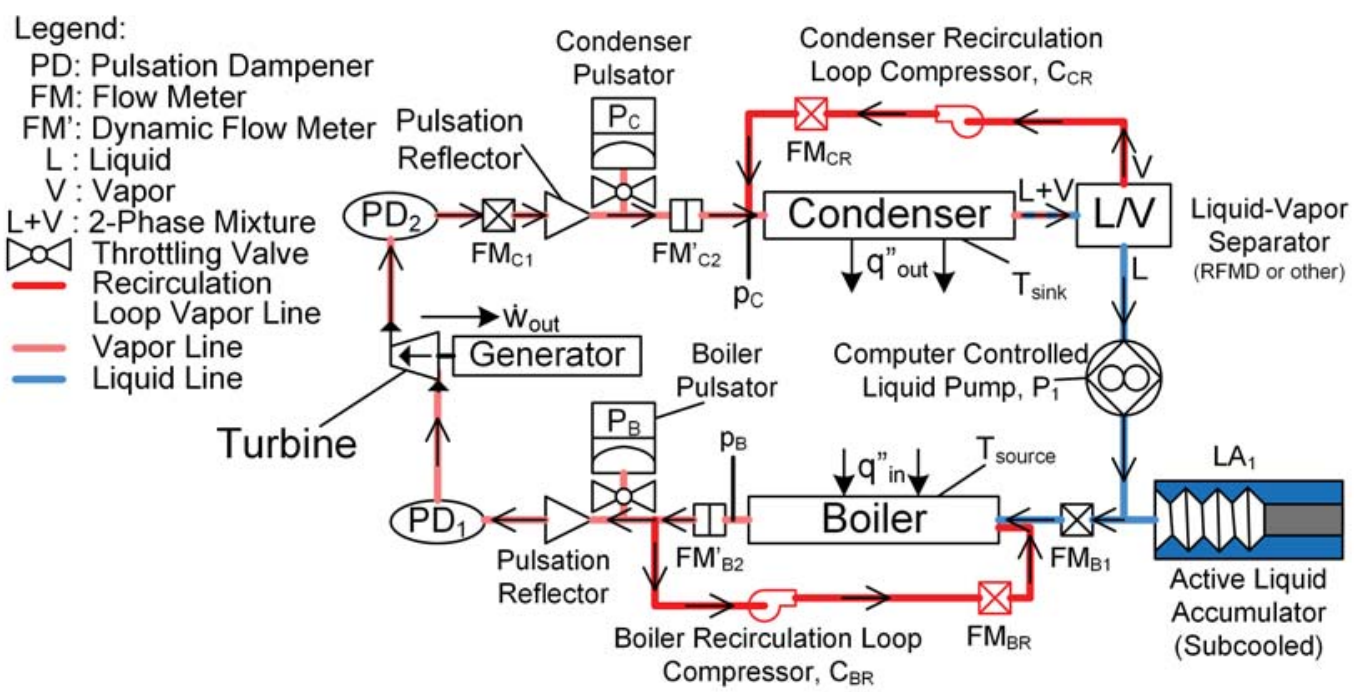

Figure 1.2. Innovative Rankin power cycle for space-based applications. For this cycle, the boiler pressure, $p_{B}$, is greater than the condenser pressure, $p_{C}$, such that heat absorption temperature, $T_{\text {source }}$, is greater than heat rejection temperature, $T_{\text {sink }}$. Energy extracted by the turbine, $\dot{w}_{\text {out }}$, from the fluid vapor as it flows from the higher pressure, $p_{B}$, to the lower pressure, $p_{C}$, drives equipment, such as the generator shown here. 
The systems in Figs. 1.1 - 1.2 have two main innovative features that enable high precision, adaptability, and robustness of their performance in the difficult micro-gravity and zero-gravity environments. First, the use of independent re-circulating vapor flow loops for the condenser and boiler allow control of the range of vapor quality within each device, enabling stabilization of the annular flow regime throughout the length of the condenser and boiler over a wide range of operating conditions. Second, the use of separate pulsators for the boiler and condenser enables the pulsations in the vapor phase to be tuned individually for each device to maximize the heat transfer enhancement realized in the boiler and condenser over a wide range of operating conditions.

The optional pulsation dampeners remove unwanted pulsations from certain flow loop components, such as the compressor of Fig. 1.1. and the turbine of Fig. 1.2. Such pulsation dampening could also be supplied to the liquid pump of Fig. 1.2 if necessary. Optional pulsation reflectors shown in Figs. $1.1-1.2$ help confine the deliberately imposed pulsations to the desired device and potentially amplify the imposed pressure pulsations. The optional liquid accumulator allows the effective charge of the refrigerant or working fluid to be adjusted, allowing the system to adapt to a wider range of source and sink temperatures. The flow meters allow system monitoring and control, in conjunction with pressure transducers (not shown in Figs. 1.1 - 1.2) measuring boiler and condenser vapor pressures $\mathrm{p}_{C}$ and $\mathrm{p}_{\mathrm{B}}$ and their fluctuations. The dynamic flow meters $\left(\mathrm{FM}_{\mathrm{C} 2}^{\prime}\right.$ and $\mathrm{FM}_{\mathrm{B} 2}$ ) and either $\mathrm{FM}_{\mathrm{C} 1}$ or $\mathrm{FM}_{\mathrm{B} 1}$ may be removed for certain non-scientific implementations of the flow loop design.

The knowledge gained from these experiments also has profound scientific value in expanding the current level of human knowledge on the interaction between the liquid and vapor phases in an internal condensing or boiling flow. This, in turn, has implications for the design of horizontal condensers and boilers in earth-based vapor compression cycle, Rankin cycle, and electronics cooling. It is expected that the principles discovered here will aid in the design of reliable and thermally efficient micro-scale boilers and, as necessary, condensers for closed loop gravity-insensitive electronics cooling systems. 
These applications provided the impetus for NSF funding of the remainder of the research reported here through grant NSF-CBET-1033591.

This dissertation establishes the reported pressure-fluctuation-induced heat-flux enhancement effect as a viable potential solution for reducing thermal resistances and providing stability of flow condensers and flow boilers operating under shear-driven flow conditions. More research is needed to quantify the improvements expected from this approach throughout the length of a flow condenser or a flow boiler and optimize its implementation. This document reinforces previous work by P. Gorgitrattanagul [5], which covered the realization and control of annular flow condensation without the imposition of upstream pressure fluctuations as reported here.

The very large heat-flux enhancements reported here for a representative location within the condenser or boiler are shown to occur over a range of frequencies at sufficiently high amplitudes of imposed inlet vapor pulsations. Moderate enhancement is inferred to occur over the entire length of the device using variations in the heat-exchange surface temperature and thermal resistance considerations for the heat-exchange surface. These enhancements are shown to occur for all-annular boiling flow and all-annular and completely condensing flows, and they have been repeated multiple times over a $2-3$ year period of time. Experiments in all-annular boiling and condensing flows used to produce the data reported in chapter 2 were performed in January, February, and June 2013. Experiments in completely condensing flow used to produce the data reported in chapter 3 were performed in June 2011.

Nikhil Shankar, in his MS thesis [6] published a schematic of a flow regime map for mm-scale 2-phase and phase-change flows. Flow regime maps like this one (included as Fig. 1.3 on the next page) should be used to guide selection of re-circulating vapor flow rates in innovative condensers and boilers such that the resulting flow is within the annular flow regime over the entire device length. It is reproduced here for purposes of commentary. 


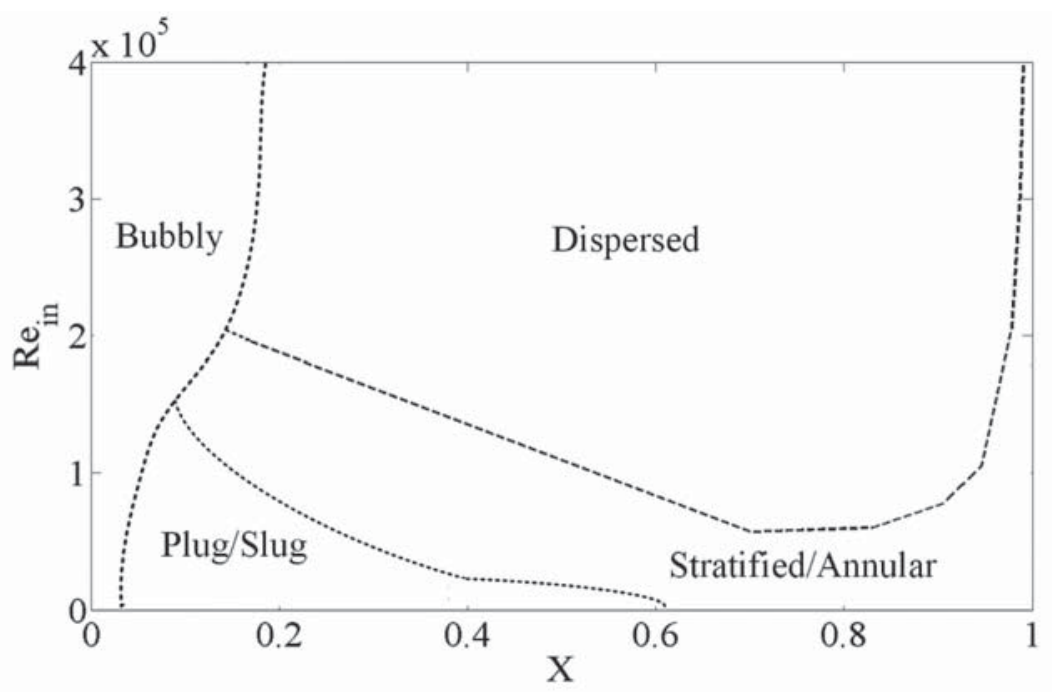

Figure 1.3. Non-dimensional flow regime schematic for two-phase flow. This figure was first published as Fig. 3.12 in [6]; it is used here for purposes of commentary.

The no-imposed fluctuations cases and most of the pulsatile flow cases for the experiments reported in chapter 2 were found to lie entirely within the stratified/annular region of Fig. 1.3 based on their ranges of inlet vapor Reynolds number, $\mathrm{Re}_{\text {in }}$, and vapor quality, $\mathrm{X}$. The annular regions of the experimental flow condensation cases reported in chapter 3 would lie within the stratified/annular region of Fig. 1.3 based on inlet vapor Reynolds number.

For pulsatile flows, based on observations in the experiments described in chapters $2-3$, it is hypothesized that this stratified/annular flow regime may be stable to lower vapor quality values than would be possible without the vapor pressure pulsations. Based on theory, it is also expected that this pulsation-induced stabilization would apply to the wavy annular flow regime (not shown in Fig. 1.3), which is sometimes reported for higher vapor Reynolds numbers. Since the flow condensation and flow boiling experiments in chapters $2-3$ provided heat-exchange to or from only the bottom surface of the horizontal flow channel, there would not be an observable difference between the stratified/annular and wavy annular flow regimes, which are simply termed 'annular' for the remainder of this dissertation. 
In summary, the influence of pulsations in the vapor pressure is to move the stratified/annular-to-bubbly and stratified/annular-to-plug/slug boundaries in Fig. 1.3 to the left for certain frequency/amplitude combinations and suitable exit conditions described in chapter 3, section 3.4.3.2.

\subsection{Pulsatile vapor flow description}

For the purposes of this document, a pulsatile flow is defined as a flow in which there are externally imposed periodic or quasi-periodic pressure or flow rate variations in time, the amplitudes of which are significant when compared with the time-averaged device pressure-difference or flow rate values, respectively. By quasi-periodic, it is meant that the most significant amplitude and frequency of the time-varying pressure or flow rate persist in their significance, although their values may vary slightly over time.

The results reported here focus primarily on the currently understood parabolic (inlet) and elliptic (exit) sensitivities as they relate to internal, horizontal condensing flows in which the incoming vapor is made pulsatile by the imposition of pressure (and corresponding flow) pulsations through specialized experimental hardware schematically represented in Fig. 1.4. What is meant by "parabolic" and "elliptic" sensitivity as they relate to the condensing and boiling flows reported here is described in sections 2.3.5 and 3.4.5. 


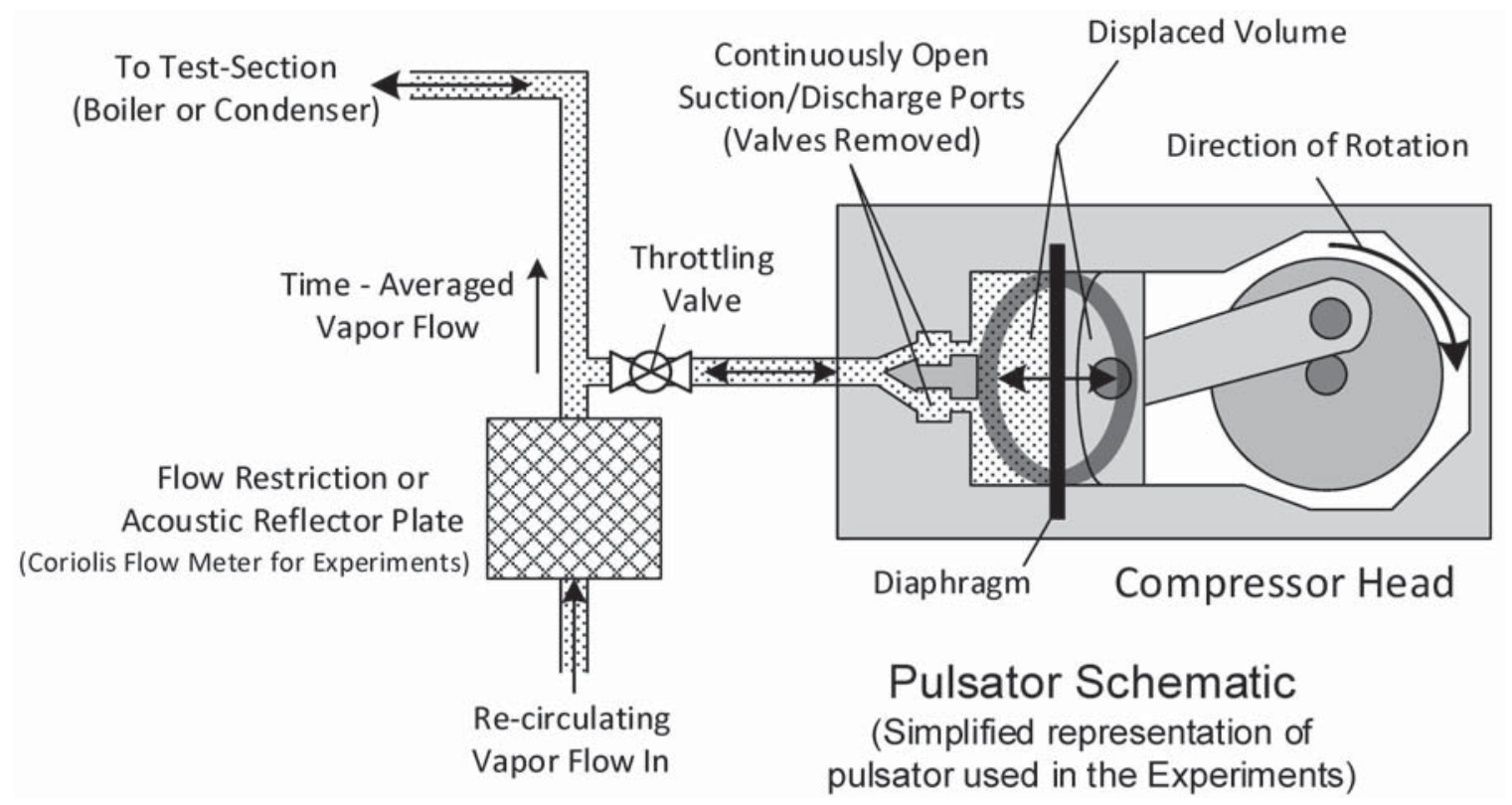

Figure 1.4. Pulsator design used in the experiments. This pulsator was modified (reed valves removed) from a commercially available diaphragm compressor head from KNF Neuberger. A variable speed 90 volt permanent magnet DC motor was used to drive the compressor. The speed of the motor controls the frequency of the pulsations, and the throttling valve limits their amplitude between zero (with the throttling valve completely closed) and a maximum (with the throttling valve completely open). The actual pulsator used in the experiments had two diaphragms in a parallel ' $V$ ' configuration, but only one diaphragm is shown here for simplicity.

Both complete and partial condensation are investigated over a range of imposed pressure fluctuation amplitudes at multiple frequencies. Additionally, preliminary experimental results for boiling flows with controlled pulsatile vapor injection, and fixed frequency and amplitude pulsatile liquid injection, at the boiler inlet are reported. The innovative experimental configurations (which use flow-through vapor to stabilize the annular flow regime throughout the device length) of the condensing and boiling flows in chapter 2 allow mechanisms contributing to the observed heat-flux enhancement phenomenon to be isolated and analyzed, specifically the ones arising from interfacial waves interacting with acoustic wave reflections from the test-section exit.

The experiments with boiling flow in a horizontal channel reported in chapter 2 showed that the parabolic sensitivity to pulsations in the vapor and liquid injection pressures (or 
flow rates) is weaker than for similar partially or completely condensing flows when the liquid film completely boils by the exit of the boiler. It is expected that future boiling flow experiments will show that the parabolic sensitivity is greater if the liquid film does not completely boil before the exit of the boiler, thereby allowing liquid-vapor interfacial waves to reflect back toward the inlet of the boiler. The difference between these two responses is due to the elliptic sensitivity of the boiling flow to the pulsations in the vapor and liquid injection pressures/flow-rates at its inlet, subject to varying types of exit liquid and vapor configurations.

\subsection{External literature review}

A large body of work has been accomplished in the fields of condensing and boiling flow. There is not space here to list most of the relevant work; the reader is referred to literature review papers such as [7 - 8]. Some examples of relevant work, arranged by topic, follow.

\subsubsection{Flow regimes}

Different liquid-vapor configurations (annular, plug/slug, bubbly, etc.) frequently arise in most gas-liquid internal flows — with phase-change (Carey [9], Collier and Thome [10], $[11$ - 12]) or without phase-change ([13 - 15]). Of these, non-annular flow regimes occur more easily and more frequently in $\mathrm{mm}-\mu \mathrm{m}$ scale ducts — both for flow condensation ([2, 16], Coleman and Garimella [18 - 20]) and flow boiling ([20], Peles et al. [11 - 12], $[21-22])$. For flow boiling and flow condensation operations in $\mathrm{mm}-\mu \mathrm{m}$ channels and under negligible gravity conditions, experimental works have also clearly established the negative impact of non-annular regimes on pressure-drop and heat-flux values.

\subsubsection{I nstabilities, instability mechanisms, and critical heat flux}

Instabilities in gas-liquid flows are of many kinds. As a flow parameter (e.g. quality)

changes, instability mechanisms control the transition of the flow from one quasi-steady 
regime (e.g. annular) to another (e.g. plug/slug). In addition, for micro-scale flow boiling ([23 - 31]) and micro- to macro-scale flow condensation ([17 - 19], [32 - 34]), internal mechanisms may couple with external system (i.e. flow loop) arrangements as in [35] to cause sustained large-amplitude time-varying flows — with or without a steady mean.

These instabilities depend on whether the flow loop arrangements are insufficiently stiff with regard to providing steady hydrodynamic boundary conditions for the device (e.g. absence of active control of the flow rates by pumps, absence of an active control - such as an inventory controller or an equivalent arrangement [2] — for steadying the mean value of pressure at one point in the flow loop). Sometimes other instabilties arise due to an overly stiff situation with regard to the ability of the surface temperatures, on both sides of the heat-exchange plate, to seek and allow themselves easily changing values. To address the last issue, low thermal inertia of the heat-exchange plate is needed to reconcile instantaneous differences between the faster changing heat load on its side exposed to phase-change flows (due to easily changing liquid-vapor configurations) and, typically, more slowly changing heat load demands on the other side exposed to externally imposed heating or cooling conditions.

The above-cited papers describe and propose several such mechanisms. For example, Leidenegg instability in [23] is said to be associated with the nature of the two "pressure-difference versus mass flow-rate" curves — one defined by the available pressure-difference across the device (as determined by pump selection, etc. in the flow loop excluding the device) and the other determined by the requirements of quasi-steady hydrodynamic operations of the flow within the device.

For flow boiling conditions, experiencing large time-varying instabilities under the prescribed heat-flux method of heating, the dry-out region becomes dangerous as it does in pool boiling ([9]). Hence the aforementioned flow boiling papers, particularly [35], often mention the influence of system factor induced instabilities on the definition of critical heat-flux (CHF) values. Clearly these "CHF" conditions are, to a certain extent, 
system-specific. These system-instability related conditions can be avoided by using flow controls to confine the boiler and condenser operation to efficient flow-regimes and by keeping heat-flux controlled heating regions always wet (e.g. using re-circulating liquid and a wetness sensor).

\subsubsection{Pumping power penalties}

For micro-scale flow boilers and condensers operating at a given mean mass flow rate, it is found that portions of the test-section experiencing quasi-steady or transient non-annular (plug/slug, bubbly, etc.) flow regimes require much larger pressure-drops $([2,16,20-22]$, etc.) than portions where the liquid and vapor phases are mostly separated as in annular flows — with or without micro-bubbles.

\subsubsection{Differences between predominantly gravity and shear driven boiling and condensing flows}

Absence of axial gravity significantly affects the behavior of flow condensation as in [36-37] and flow boiling as in [38]. As inclined ducts are made horizontal to vertical, for condensing flows, the effect of changes in inclination angle goes away as hydraulic diameter decreases (see Mitra et al. [36]) and gravitational force decreases relative to other forces (inertia, shear/viscous, etc.). In [36 - 37], our experiments and theory show that gravity-driven internal condensing flows are quite stable, mostly annular, and predictable (by using the tools we have developed for this purpose).

\subsubsection{Enhancement approaches for pool and flow boiling - texturing of the heat-exchange surface (solid-liquid interface) and active utilization of enhanced heat-flux values at multiple contact-line-type thin film locations}

In the literature available for mm or higher length-scale flow boilers, micro-fins [39-40], porous-surfaces [41 - 42], meshes and screens [43 - 44], micro-channels

within such channels (see Liu and Garimella [45 - 46], Peles et al. [47 - 49]), re-entrant grooved micro-structures [48 - 49], etc., have been used to demonstrate enhanced 
heat-flux due to enhanced nucleation. For most such enhanced flows, unacceptable pressure-drop (and pumping power) penalties or large transient instability issues remain unresolved.

Classical nucleation studies in liquid pools as in [9] or within a liquid drop (Chandra and Avedisian [50]) have been extended to promote nucleation by exploiting chemical, electro-chemical, and nano-meter scale structuring ([51 - 54]) approaches for manipulating the wettability of heat-exchange surfaces. Similarly, there have been flow boiling studies where nano-meter scale texturing is done with the help of nano wires ([55 - 56]), carbon nano tubes ([57 - 58]), use of nano fluids for deposition of nano-particulates on the heater-surface ([59]), etc. However, in most such nucleation promotion based heat-flux enhancement studies, the issues of inefficient flow regimes, large transient instabilities, and long-term durability of the heat-exchange surfaces (cleanliness/fouling issues) are not directly or fully addressed.

For $\mathrm{mm}$ or higher scale flow condensers, film condensation typically involves $\mu \mathrm{m}$-scale liquid films. Therefore $\mu \mathrm{m}$-scale surface-texturing is typically not recommended for internal flows. However, to promote dropwise condensation during quiescent vapor condensation on inclined or horizontal surfaces, chemical and nano scale texturing has been employed to produce highly hydrophobic surfaces that not only cause droplets to appear and move but also cause them to bounce around during coalescence ([60 - 61], etc.).

During a nucleating bubble's growth and departure, very high heat-fluxes are observed (Stephan et al. [62 -63]) at the advancing and receding contact line regions. These occur within the thin liquid film micro-layers (nano-meter to sub-micro-meter thick) that form the contact line regions [63]. Other issues discussed in the literature include detailed experimental investigation of nucleate boiling mechanisms ([64 - 65]), molecular scale simulations (Maroo and Chung [66], Wemhoff and Carey [67], etc.) that investigate the role of adsorbed layers (typically 1-10 nm thick) and disjoining pressures within 
the adsorbed region and the adjacent micro-layer $([62-63,68])$, investigation of the disjoining pressure's role for macro-scale computational modeling ([62 - 63]) of flow near the contact lines, etc. These studies have a strong bearing on the proposed investigation of mechanisms associated with the very high heat-flux enhancements observed in $[2,16,20]$ and for the innovative mode of operation of the proposed flow boilers and flow condensers.

The main qualitative understanding of these phenomena, as described in $[62-63,68]$, is that the wetted continuum region does not abruptly end at the contact line where the so-called "dry" region begins. Instead, the continuum macro-layer first thins to a small length of stationary or moving micro-layer (say $200 \mathrm{~nm}-1 \mu \mathrm{m}$ in thickness) associated with a stationary or moving contact line which then merges with a typically longer molecular thickness zone (e.g. $1 \mathrm{~nm} \sim 10 \mathrm{~nm}$ thick) which is referred to as the adsorbed layer in $[62-63,68]$. The molecules in the adsorbed layer stick to the solid due to the relatively stronger Van der Waals forces over the so-called “dry” surface region.

The pressure and other stresses in this adsorbed layer can be either compressive or tensile - much like a solid - except that the actual pressure and stress levels within the adsorbed layer exhibit metastable spatial and temporal variations determined by the flow requirements in the adjacent micro-layer (see [63]), which in turn are determined by requirements of the flow physics in the macro-layer.

For example, for advancing and receding contact lines associated with boiling/evaporating fronts as in [63], disjoining pressure effects in the adsorbed layer may sufficiently lower the effective pressure in the micro-layer to allow the necessary half-circulation flow patterns [63] required for the micro-layer.

\subsubsection{Modeling and correlations}

Besides the relevant results mentioned above, for the general area of condensing and boiling flows, there is excellent available knowledge with regard to exact and 
approximate model equations for $\mathrm{mm}-\mu \mathrm{m}$ scale flows and conditions at the interface (Carey [9], Delhaye [69], Faghri et al. [70 - 72], etc.). These equations can be used and are being used for first-principles based CFD simulations (as well as CFD for averaged equations) for certain regimes of nucleate boiling (Mukherjee and Dhir [73]), flow boiling [74 - 75], and flow condensation [76 - 80]. Our own CFD capabilities for annular boiling [20] and annular condensation [76 - 80] have recently achieved (as described later) unprecedented accuracy levels - both from a computational/theoretical point of view of accurately capturing wave dynamics and with regard to compatibility with experimentally obtained data.

For internal flow boiling, it is customary to propose approximate heat-transfer coefficient and CHF correlations [81 -83]. For flow condensation, key literature can be roughly summarized/categorized as: (i) classical solutions for external film condensation flows over vertical, horizontal, and inclined walls (Nusselt [84], [85 - 86], etc.) and their subsequent modifications and extensions ([87 - 90], etc.); and (ii) experimental data and correlation for heat transfer rates for the various external condensing flow regimes ([91 - 93], etc.) as well as internal condensing flows ([94 - 99], etc.).

\subsubsection{Phase-change pulsatile flow experiments}

Very little work has been done on researching the effect of vapor phase pressure (or flow) pulsations on heat-transfer rates and pressure drops for internal condensing or boiling flow outside of the work reported here and some of the preceding investigations of sections 1.5.1.3, 1.5.2.3, 1.5.3.1, and 1.5.4. Of the available work by other researchers, experimental investigations of C. W. Roh and M. S. Kim ([100]) on the impact of periodic flow perturbations on a vapor compression cycle are particularly noteworthy.

In [100], C. W. Roh and M. S. Kim provide a solenoid-actuated valve in parallel with the expansion valve of an experimental vapor compression cycle. This solenoid valve was opened periodically for time-periods of 0.2 seconds at various cycle time-intervals from 5 seconds to 200 seconds. Thus the percentage of the cycle time that the valve was 
open varied from $4 \%$ for the 5 second time-period to $0.1 \%$ for the 200 second time-period.

Note that the flow configuration of [100] allowed for significant effects of gravity on the liquid flow in the condenser and evaporator, in contrast to the horizontal channel flows presented in chapters $2-3$. Also, no provision for phase-separation at the inlet of the evaporator (downstream of the expansion and control valves of Fig. 2 in [100]) was provided in [100], in contrast to the separator plate of Fig. 2.3b in chapter 2, so the flow into their evaporator likely contained suspended liquid droplets.

The effects of these non-sinusoidal flow pulsations on the cycle were analyzed and divided into two categories: enhancement of heat transfer by pulsatile flow and system-level phenomena termed "cycle elevation" and "pushing" in [100]. The "cycle elevation" and "pushing" effects relate to the thermodynamic cycle analysis of the vapor compression cycle and the influence of pressure excursions in the condenser and evaporator due to the action of the solenoid valve. It suffices to note here that some of the adverse cycle effects observed by Roh and Kim as a result of their imposed flow pulsations may be reduced or eliminated in suitable pulsatile flow configurations similar to those presented in Fig. 2.1b and Fig. 2.2b of chapter 2. Only the first category, enhancement effects, is relevant to the research presented here, which focuses specifically on the heat-flux enhancement within the flow condenser or flow boiler.

The heat-flux enhancement effect was concluded in [100] to be consistent with a combination of turbulence effects and cavitation at the tube wall (specifically applicable to boiling/evaporating flows). The enhancement mechanisms presented in sections 2.3.5 and 3.4.5 of this dissertation come from a completely different source. However, the effect of vapor core turbulence induced by vapor pressure (or flow) pulsations on heat-transfer enhancement in shear/pressure-driven internal condensing and boiling flows is acknowledged in chapter 4 as a subject which deserves further investigation, mainly for computational modeling purposes. 


\subsection{Research conducted at Michigan Technological University}

The research referenced in this section is from the author's faculty adviser and research group at Michigan Technological University. It forms the basis for the work presented later in this dissertation and provides a temporal context for that work.

\subsubsection{Elliptic shear/ pressure-driven internal condensing/ boiling flow hypothesis development}

This section covers the history of the current theory on the concept of "elliptic sensitivity," or concurrent inlet and exit condition dependency, of internal condensing and boiling flows. This work was developed by Prof. Amitabh Narain's research group over 19 years of computational and experimental investigations. Effort is made to preserve the chronological order and logical development leading to the current hypothesis, which is very briefly summarized in section 1.5.1.4; therefore, some parts of this section (1.5.1) are redundant with sections 1.5.2 - 1.5.4, where more details of the experiments are presented.

\subsubsection{Early computational work}

In 1996 A. Narain published a paper in which he reported a modeling approach for annular film condensation [101]. The next year he published an article with G. Yu and Q. Liu expanding the analysis to inclined channels and vertical pipes [102]. Based on this work, a two-dimensional computational simulation tool was developed and reported by A. Narain, Q. Liang, G. Yu, and X. Wang in 2004 [76] for annular condensing films within a vertical channel. It is here that the concept of exit condition effects on the condensing flow inside channels was first introduced. The simulation method used at that time indicated a multiplicity of solutions to the problem for the same inlet pressure, inlet mass flow rate, and wall temperature conditions, each corresponding to a different exit pressure or vapor quality (for partially condensing flows).

Nevertheless, it was recognized in [76] that an "attractor" or "natural" solution to the 
condensing flow problem exists for a given set of inlet pressure, inlet mass flow rate, and wall temperature conditions which is stable (based on analysis of 2-dimensional imposed disturbances) in the absence of an imposition on the exit pressure or vapor quality. It was also computationally shown in [76] that miniscule vibrations of the condensing surface in the $0-30 \mathrm{~Hz}$ range may have a significant influence on the waviness of the liquid-vapor interface and mean heat flow rates if they couple with the frequency associated with the time-evolution of imposed interfacial disturbances, leading to a resonance condition within the liquid film.

An expansion of the computational methodology of [76] to include tilted and horizontal channel geometries, as well as zero-gravity channel condensation was reported in [80]. Also, in [80] shear and tangential surface tension effects were investigated in more detail, with $\sigma=\sigma(T)$, with surface tension effects becoming significant only in the direction normal to the interface, and only for the zero-gravity cases investigated. In zero-gravity conditions, it was noted that the surface tension has a limiting effect on the amplitude of interfacial waves which arise from initial disturbances and wall vibrations.

From the then-current computational model, it was found in [80] that the solutions were quite insensitive to noise (in the form of initial disturbances) in the vapor flow at the inlet under unconstrained ("natural”) exit conditions. L. Phan, X. Wang, and A. Narain extended their analysis with this computational tool and published the results in [78], in which they first stated directly the hypothesis that multiple exit conditions were possible for shear and gravity-driven condensing flow, and that for shear-driven condensing flows there would be a "loss of parabolicity," which would occur at some point before the flows fully condensed. This meant that shear-driven (horizontal or zero-gravity) flows which were condensed completely or sufficiently to pass this transition point would not have a "natural" exit condition and would require exit condition (pressure or vapor quality) constraint. 


\subsubsection{Gravity-driven flows shown to be parabolic in nature}

The existence of the hypothesized multiple exit conditions appeared to be supported by experiments published by A. Narain, J.H. Kurita, M. Kivisalu, A. Siemionko, S. Kulkarni, T.W. Ng, N. Kim, and L. Phan the next year in [103] in which exit vapor quality constraints appeared to be imposed on a condensing flow in a vertical tube condenser. However, these results could not be repeated when a more reliable experimental system and procedures were used which limited the levels of non-condensable gasses in the working fluid to a repeatable and low level.

These experiments were carried out by J.H. Kurita and M. Kivisalu, and they were reported by J.H. Kurita, M. Kivisalu, S. Mitra, R. Naik, and A. Narain in [37], in which it was concluded that the originally hypothesized elliptic response of condensing flows was limited to certain shear-driven flow conditions. Details of the computational code are presented by S. Kulkarni in his dissertation [104].

A bug in the computational code was shown in [79] to be responsible for the computational results indicating the attainability of multiple exit conditions for the same inlet and wall temperature conditions in gravity dominated condensing flow and in steady flow predictions for shear/pressure-driven flows. It was acknowledged in [79] that even quasi-steady shear/pressure-driven flows, if given inlet and condensing surface cooling conditions, will seek a single value of exit pressure, termed "natural" in [79]. This paper stated that the "elliptic sensitivity" phenomenon was due to the unsteady nature of the pulsatile shear/pressure-driven flows, which could be captured by unsteady simulations.

\subsubsection{Shear/pressure-driven unsteady, non-pulsatile flows found to be parabolic in nature}

Experiments on condensing flow in a horizontal channel, reported in [79], initially appeared to indicate elliptic behavior when a diaphragm compressor was used to help constrain the condenser inlet pressure while its exit pressure was held fixed by a feedback controlled liquid pump. However, afterwards it was found that shifts in the wall 
temperature between the cases, initially assumed to be negligible, actually rendered the supposed "constrained" pressure-difference cases similar to other "natural" cases from the simulation results.

This was verified when the experiments were repeated with the diaphragm compressor converted to supply only pulsations to the inlet vapor flow rate, with a separate compressor (vane-type, with pulsation dampening on its discharge) supplying the compression of the vapor. These experiments are reported in [105], [16]. Further revisions to the computational code were reported by S. Mitra in his dissertation [106], in which the concept of "elliptic sensitivity" for unsteadiness in shear/pressure-driven flows is omitted completely.

\subsubsection{Elliptic sensitivity discovered in pulsatile shear/pressure-driven condensing flows}

Around this time it was discovered that large enhancements in the mean heat-flux were measured at a location within the heat-exchange surface of the condenser when the compressor was operating. It was hypothesized that there might be a combination of "parabolic" and "elliptic" sensitivities of the local heat flux and liquid film morphology to the pulsations induced by the diaphragm compressor.

The "parabolic" sensitivities to inlet pressure pulsations indicate a one-to-one response of the annular regime interface configuration and heat-flux levels to the level of imposed inlet pressure or flow pulsations. The "elliptic" sensitivity was isolated by drawing down the liquid from the exit of the horizontal condenser into a vapor plenum, both providing damping for the acoustic vapor pulsations and making it more difficult for interfacial liquid waves to reflect back toward the inlet of the condenser. It was found that, if there was a sufficiently large column of vapor at the exit of the condenser, the heat-flux enhancement would be much reduced or nonexistent at the measurement location, even for similar levels of inlet vapor pressure pulsations (experimental data not published). Because the physical configuration of the exit of the condenser has an influence on 
upstream mean and fluctuating heat-flux for partially condensing flow, all other flow conditions being the same, it may be said that an "elliptic sensitivity" to inlet vapor pressure pulsations has been experimentally observed for these flows.

\subsubsection{Current understanding on elliptic sensitivity in shear/pressure-driven pulsatile condensing and boiling flows}

As explained in chapters $2-3$, it is now known that the "elliptic sensitivity" effect for pulsatile shear/pressure-driven condensing and boiling flows is due to effects resulting from the interaction of "standing" acoustic waves with the liquid-vapor interface. The high amplitude of the induced vapor velocity fluctuations within the standing waves encourage large amplitude fluctuations in the location of the liquid-vapor interface that allow the interfacial wave troughs to come close enough to the heat-exchange surface (e.g. the bottom surface of the flow channel for experiments reported in this dissertation) to allow solid-liquid-vapor effects to preferentially bias the interfacial fluctuations toward the heat-exchange surface. Details are discussed in sections 2.3.5 and 3.4.5.

The ability to form standing acoustic waves within the vapor flow field is dependent on the ability of the exit of the condenser or boiler to reflect the acoustic wave energy back over the annular flow regime. This exit condition sensitivity phenomenon is not directly caused by unsteadiness in the flow, as previously thought. Rather, it is due to the complex interaction of forward and backward moving acoustic waves with the liquid-vapor interface and the corresponding complex interaction of the liquid-vapor interface with the heat-exchange surface at multiple length and time scales, as discussed in section 2.3.5.

\subsubsection{Horizontal flow condenser performance}

\subsubsection{First attempts of horizontal condensing flow experiments at Michigan Technological University}

The first horizontal flow condensation experiments were performed by Q. Lu and N. V. Suryanarayana for a $4 \mathrm{~cm}$ wide by $2.5 \mathrm{~cm}$ high by $100 \mathrm{~cm}$ long flow channel for refrigerants R113 and FC-72. Adiabatic air-water flows were also investigated for 
comparison purposes. The results were published by Q. Lu in his doctoral dissertation in 1992 ([107]). In 1995, similar experimental results were reported in the ASME Journal of Heat Transfer by Q. Lu and N. V. Suryanarayana ([108]).

Professor A. Narain, upon finding that these experimental results did not agree with computational simulations for horizontal condensing flow, but they agreed with computational simulations for flows with a $1-2$ degree incline downward in the direction of flow, confirmed from Prof. N. V. Suryanarayana that Q. Lu had verified only that the top surface of the flow channel was horizontal, and that the bottom surface might have been slightly inclined. This was reported by S. Mitra in his doctoral dissertation ([106]).

In short, these experiments are not characteristic of horizontal channel flows; rather, they represent flows in channels that have a slightly downward incline in the direction of the flow. Therefore, they exhibit a mixture of gravitational and shear/pressure influences in driving the liquid along the condenser ("mixed" driven flow of [106]).

\subsubsection{Condensation experiments conducted in 2008 - 2010}

Early experiments on a similar horizontal flow condenser to the one used in the experiments of chapters 2 and 3 were conducted on September 2, 2008 with the $6 \mathrm{~mm}$ high by $24 \mathrm{~mm}$ wide by 1 meter long test-section geometry of Fig. 2.3a, but without the heat-flux meter or reliable pressure-difference measurements. These experiments were for visualization purposes only. They resulted in a conference presentation ([109]) but were not published as a paper in the conference proceedings.

Due to reliability problems with the $6 \mathrm{~mm}$ high channel and changes in the design of the experiments, the $6 \mathrm{~mm}$ high test-section was replaced with a $2 \mathrm{~mm}$ high by $15 \mathrm{~mm}$ wide by 1 meter long channel as in Fig. 3.1a. The first experiments in this channel with flow visualization were performed by December 13, 2009, and more extensive experiments 
were performed using this flow channel on January 22 - 23, 2010 and February 4-6, 2010. However, these experiments were not published due to insufficient instrumentation and flow controls, coupled with the higher priority of publishing the gravity-driven flow condensation experiments of [37] and [110], which were being run during 2008 - 2009.

\subsubsection{Complete condensation experiments conducted in early 2011}

M. Kivisalu, N. Gorgitrattanagul, S. Mitra, R. Naik, and A. Narain first reported data acquired on March 11, 2011 from complete flow condensation in the test-section of Fig. 3.1a at the ASME International Mechanical Engineering Congress and Exposition in Denver, CO ([105]). These experiments utilized the same procedures for imposing pressure/flow pulsations as are reported in chapters $2-3$, and they used very similar flow control strategies to those presented in chapter 3.

Fully condensing flow data in this test-section acquired on March 18, 2011, with slightly different flow controls, was reported by M.T. Kivisalu, P. Gorgitrattanagul, S. Mitra, R.R. Naik, and A. Narain [16]. These experiments used feedback control of a liquid pump to set the exit pressure of the condenser, and they concurrently applied pulsations (with a "pulsator" device described in [16]) to the vapor flow supplied to the condenser while increasing the speed of a compressor in attempts to "push" the condenser inlet pressure to different quasi-steady values. It was found in [16] that the use of the compressor did not affect pressure-drop values within the condenser more than using the pulsator alone.

\subsubsection{Complete condensation experiments conducted in June 2011}

In June, 2011, a significant data matrix of pulsatile, horizontal, completely condensing flows were acquired under 2 cooling conditions for a variety of pressure pulsation amplitudes at four different frequencies. This data is reported in chapter 3 (originally published as [2]). Data collected on June 15 - 16, 2011 used the "passive" cooling condition described in section 3.3.3, and data collected on June 18 - 19, 2011 used the "mixed" cooling condition described in section 3.3.3 (section 3.3 of [2]). 


\subsubsection{Complete condensation experiments conducted in late 2011}

In 2011, Patcharapol Gorgitrattanagul published a masters' thesis titled "The length of the annular regime for condensing flows inside a horizontal channel - the experimental determination of its values and its trends" [111]. These experiments did not include deliberately imposed fluctuations/pulsations in the vapor pressures in contrast to the condensing flow results reported here in chapters $2-3$; however, they established a range of operating conditions under which stable annular flow could be achieved.

\subsubsection{All-annular condensation experiments conducted in January 2013}

On January 30 - 31, 2013, M. Kivisalu and P. Gorgitrattanagul performed experiments involving incomplete horizontal flow condensation in which the non-condensed vapor at the exit of the condenser was re-circulated and combined with the condenser inlet vapor stream using a compressor. Annular flow was achieved throughout the entire length of the device, and pressure/flow pulsations were imposed at various amplitudes for two different frequencies. The pulsation-induced heat-flux enhancement previously observed for horizontal completely condensing flows was successfully obtained for the all-annular flow condenser. The results are reported in chapter 2 (originally published as [1]).

\subsubsection{Horizontal flow boiler performance}

\subsubsection{Flow boiling experiments conducted in 2012}

A horizontal flow boiler of $1.6 \mathrm{~mm}$ gap height, $15 \mathrm{~mm}$ width, and approximately 0.72 meter length was constructed out of glass-filled Garolite phenolic composite (G-11). The top of the flow channel was constructed out of a tempered glass window to allow flow visualization, and heat was supplied from the bottom by seven copper blocks of $15 \mathrm{~mm}$ width and $100 \mathrm{~mm}$ length which were exposed to the liquid flow. Surface temperatures of the copper blocks were used as feedback to control the AC electrical power supplied to the embedded cartridge heaters in each copper block.

Flow arrangements allowed all liquid or a mixture of liquid and vapor to be supplied 
to the boiler inlet, although there was not provision for separation of the phases up to the start of flow boiling, as in the test-section of Fig. 2.2b. Pressure pulsations could be supplied to the inlet or exit vapor flow, but the pulsator (see description in section 2.2.1 or [1]) was located far away from the test-section, so the range of amplitudes of pressure/flow pulsations that could be applied to the test-section inlet or exit was limited. At that time the effects of pulsations in the liquid flow rate supplied to the boiler and their interactions with the vapor phase pulsations were not recognized; in chapter 2 it is shown that matching the inlet liquid and vapor pulsation frequencies is an important condition for attaining pulsation-induced heat-flux enhancement within a horizontal flow boiler.

Experiments in this test-section were conducted on January 10 - 11, 2012 regarding stabilization of the annular flow regime using re-circulating vapor flow, and they were successful at achieving mostly annular boiling flow throughout the length of the test-section or until dry-out, when it occurred within the flow boiler. Representative flow regime pictures in Fig. 2.1b and Fig. 2.2b were taken from these experiments.

Experiments conducted on January 21 - 22, 2012 attempted to realize the heat-flux enhancement previously observed for completely condensing flows (see [16], [2]). They used the same heat-flux sensor, positioned near the middle of the boiler length, but they were unable to demonstrate any significant enhancement in time-averaged heat-flux values at the range of pulsation frequencies and amplitudes that were investigated. Possible reasons for this have been mentioned in the previous paragraph. The results of these experiments were reported in [20]. Note that some incorrect concepts of the flow physics presented in this conference paper have been rectified in [1] and chapter 2, which contain more useful flow boiling experimental results with regard to pulsatile flows.

\subsubsection{Flow boiling experiments conducted in 2013}

On February 17 - 18, 2013, M. Kivisalu and P. Gorgitrattanagul performed experiments involving incomplete horizontal flow boiling in which mechanically separated streams of liquid and vapor were supplied to the inlet of the flow boiler, and all of the liquid 
was boiled within the length of the device. A portion of the boiler exit vapor stream was diverted and re-circulated by means of a compressor to supply the inlet vapor flow rate. Pressure (or flow) pulsations were supplied to this inlet vapor stream at various amplitudes for two different frequencies. Mostly annular flow was achieved throughout the entire length of the device, and the pulsation-induced heat-flux enhancement previously observed for horizontal completely and partially condensing flows was successfully obtained for the all-annular flow boiler (see chapter 2 or [1]).

For these experiments, it was found that significant pulsations in liquid flow rates supplied to the boiler were inadvertently imposed. Although these pulsations did not significantly affect the time-averaged pressure-differences or heat-flux, they did affect the ability to obtain the pulsation-induced heat-flux enhancement phenomenon. Equal or compatible frequencies of pulsations supplied to the inlet vapor flow led to heat-flux enhancement, but incompatible vapor pulsation frequencies did not. Select experimental results leading to the heat-flux enhancement are reported in chapter 2 (or [1]).

On June 14 - 15, 2013, similar horizontal flow boiling cases were obtained by M. Kivisalu and P. Gorgitrattanagul for a wider range of imposed pulsation frequencies and inlet liquid and vapor time-averaged flow rates. Select experimental results are reported in chapter 2 (originally published as [1]).

\subsubsection{Gravitational influence}

Vertical (gravity-driven) condensing flows were experimentally investigated by J. Kurita. Publication of the results occurred in 2007 and 2011. This work was compared with shear/pressure-driven flows in the 2011 publications. The main relevance of this work is in its comparisons between gravity-driven and shear/pressure-driven flows, but the 2007 publications formed the basis for the research that produced the 2011 publications.

\subsubsection{Experiments conducted in 2007}

In 2007, J. H. Kurita and M. Kivisalu ran experiments in the flow loop built by 
A. Siemionko. In these experiments, a vertical tube-in-tube counter-flow heat exchanger (test-section) was used to condense FC-72 vapor in the center tube, with water providing cooling in the annulus. Computational investigations were performed by S. D. Kulkarni using codes developed by N. Kim and L. Phan. The arrangement of the boroscope and fiber-optic based flow visualization equipment for the test-section had been developed by T. W. Ng. The work was published by A. Narain in the ASME Journal of Heat Transfer [103], and the results appeared to indicate that multiple exit vapor qualities (equivalent to the specification of multiple exit pressures) were possible for the test-section subject to the same inlet pressure, inlet mass flow rate, and cooling condition. This work also led to J.H. Kurita's master's thesis ([112]).

\subsubsection{Changes in experimental hardware and procedures between 2007 and 2011}

Between 2007 and 2011, it was found that the experimental flow loop used in [103] and [112] typically had unacceptably large and inconsistent concentrations of noncondensable gasses from the ambient air dissolved in the FC-72 working fluid. It was found that this was caused by a combination of operating the system at vacuum pressures with leaks (mostly in the test-section, which was made of a mixture of aluminum and stainless steel and had many ports for instrumentation) and inadequate start-up procedures.

The leak problems were remedied by replacing the test-section with a new one of the same inner tube geometry, but made entirely of welded stainless steel sections. Also, the geometries of the water flow area and probe ports were significantly simplified. New startup procedures were developed which included a "purge" process in which the noncondensable gasses were removed from the FC-72 in the flow loop to repeatable and acceptably low levels by the application of a vacuum to the highest point in the flow loop for a specified amount of time. Details of the new test-section and purge process were not published. 


\subsubsection{Experiments conducted in 2011}

Experiments performed after the improvements to the flow loop were unable to repeat the multi-valued exit vapor qualities observed in [103] and [112]. These results were reported in the ASME International Journal of Heat and Mass Transfer by J. H. Kurita and M. Kivisalu who ran the experiments, S. Mitra and R. Naik who provided computational simulations for comparison with the experimental results and with shear/pressure-driven flows, and A. Narain who developed the theory and hypotheses ([37]). These results were also published by J. H. Kurita in his doctoral dissertation ([110]).

It is important to note that the compressor used in the experiments reported in chapter 9 of [110] was a diaphragm type which introduced significant pulsations to the vapor flow. With this same compressor, experiments with shear/pressure-driven flow from December 2009 - February 2010 showed significant influence of the compressor fluctuations on the time-averaged heat-flux at a representative location within the test-section. Although the pressure-difference data were not reliable for these initial shear/pressure-driven experiments, later pulsatile horizontal flow condensation experiments performed in 2011 suggested that the time-averaged pressure-differences change along with the time-averaged heat-flux when large amplitude pressure/flow pulsations are imposed on shear/pressure-driven fully and partially condensing flows as explained in chapters $2-3$. This behavior was not observed for the gravity-driven pulsatile condensing flows investigated in chapter 9 of [110]. 


\section{References: chapter 1}

[1] M.T. Kivisalu, P. Gorgitrattanagul, A. Narain, Results for high heat-flux flow realizations in innovative operations of milli-meter scale condensers and boilers, International Journal of Heat and Mass Transfer, 75 (2014) 381-398.

[2] M.T. Kivisalu, P. Gorgitrattanagul, A. Narain, R. Naik, M. Hasan, Sensitivity of shear-driven internal condensing flows to pressure fluctuations and its utilization for heat flux enhancements, International Journal of Heat and Mass Transfer, 56(1-2) (2013) 758-774.

[3] C.J.M. Lasance, R.E. Simons, Advances In High-Performance Cooling for Electronics, in: Electronics Cooling, (2005).

[4] P.E. Phelan, V.A. Chiriac, T.-Y.T. Lee, Current and future miniature refrigeration cooling technologies for high power microelectronics, IEEE Transactions on Components and Packaging Technologies, 25(3) (2002) 356-365.

[5] P. Gorgitrattanagul, The Length of the Annular Regime for Condensing Flows Inside a Horizontal Channel - The Experimental Determination of Its Values and its Trends, M.S. Thesis, Michigan Technological University, Houghton, 2011.

[6] N. Shankar, An Assessment of Flow Regime Maps and a Numerical Heat Transfer Correlation for the Stratified/Annular Regime of Shear-Driven Internal Condensing Flows, M.S. Thesis, Michigan Technological University, Houghton, 2014.

[7] S.-M. Kim, I. Mudawar, Review of databases and predictive methods for pressure drop in adiabatic, condensing and boiling mini/micro-channel flows, International Journal of Heat and Mass Transfer, 77 (2014) 74-97.

[8] S.-M. Kim, I. Mudawar, Review of databases and predictive methods for heat transfer in condensing and boiling mini/micro-channel flows, International Journal of Heat and Mass Transfer, 77 (2014) 627-652.

[9] V.P. Carey, Liquid-Vapor Phase-Change Phenomena, Series in Chemical and Mechanical Engineering, Hemisphere Publishing Corporation, 1992.

[10] J.G. Collier, J.R. Thome, Convective Boiling and Condensation, 3rd ed., Oxford University Press, Oxford, England, 1994.

[11] C.J. Kuo, Y. Peles, Flow Boiling of Coolant (HFE-7000) Inside Structured and Plain Wall Microchannels, Journal of Heat Transfer, 131(12) (2009) 121011-121019.

[12] Y. Peles, Two-Phase Boiling Flow in Microchannels-Instabilities Issues and Flow Regime Mapping, in: 1st International Conference on Microchannels and Minichannels, Rochester, NY., (2003).

[13] Y. Taitel, A.E. Dukler, A model for predicting flow regime transitions in horizontal and near horizontal gas-liquid flow, AIChE Journal, 22(1) (1976) 47-55. 
[14] P.M.Y. Chung, M. Kawaji, The effect of channel diameter on adiabatic two-phase flow characteristics in microchannels, International Journal of Multiphase Flow, 30(7-8) (2004) 735-761.

[15] S. Saisorn, S. Wongwises, Flow pattern, void fraction and pressure drop of two-phase airwater flow in a horizontal circular micro-channel, Experimental Thermal and Fluid Science, 32(3) (2008) 748-760.

[16] M. Kivisalu, P. Gorgitrattanagul, S. Mitra, R. Naik, A. Narain, Prediction and Control of Internal Condensing Flows in the Experimental Context of their Inlet Condition Sensitivities, Microgravity Sci. Technol., 24(3) (2012) 147-155.

[17] J.W. Coleman, S. Garimella, Two-phase flow regimes in round, square and rectangular tubes during condensation of refrigerant R134a, International Journal of Refrigeration, 26(1) (2003) 117-128.

[18] H.Y. Wu, P. Cheng, Condensation flow patterns in silicon microchannels, International Journal of Heat and Mass Transfer, 48(11) (2005) 2186-2197.

[19] X. Quan, P. Cheng, H. Wu, Transition from annular flow to plug/slug flow in condensation of steam in microchannels, International Journal of Heat and Mass Transfer, 51(3-4) (2008) 707-716.

[20] A. Narain, M. Kivisalu, R. Naik, N. Gorgitrattanagul, S. Mitra, M.M. Hasan, Comparative Experimental and Computational Studies for Annular Condensing and Boiling Flows in Millimeter Scale Horizontal Ducts, in: Proceedings of the ASME 2012 Summer Heat Transfer Conference, Rio Grande, Puerto Rico, (2012).

[21] P. Balasubramanian, S.G. Kandlikar, Experimental Study of Flow Patterns, Pressure Drop, and Flow Instabilities in Parallel Rectangular Minichannels, Heat Transfer Engineering, 26(3) (2005) 20-27.

[22] S.G. Kandlikar, Fundamental issues related to flow boiling in minichannels and microchannels, Experimental Thermal and Fluid Science, 26(2-4) (2002) 389-407. [23] T. Zhang, T. Tong, J.-Y. Chang, Y. Peles, R. Prasher, M.K. Jensen, J.T. Wen, P. Phelan, Ledinegg instability in microchannels, International Journal of Heat and Mass Transfer, 52(25-26) (2009) 5661-5674.

[24] L. Tadrist, Review on two-phase flow instabilities in narrow spaces, International Journal of Heat and Fluid Flow, 28(1) (2007) 54-62.

[25] D. Brutin, L. Tadrist, Destabilization Mechanisms and Scaling Laws of Convective Boiling in a Minichannel, Journal of Thermophysics and Heat Transfer, 20(4) (2006) 850-855. 
[26] J. Barber, K. Sefiane, D. Brutin, Two Phase Boiling and Flow Instabilities in a Microchannel, in: Proceedings of International Conference on Nanochannels, Microchannels and Minichannels, Puebla, Mexico, (2007).

[27] S. Kakac, B. Bon, A Review of two-phase flow dynamic instabilities in tube boiling systems, International Journal of Heat and Mass Transfer, 51(3-4) (2008) 399-433.

[28] T. Harirchian, S.V. Garimella, Effects of channel dimension, heat flux, and mass flux on flow boiling regimes in microchannels, International Journal of Multiphase Flow, 35(4) (2009) 349-362.

[29] P. Cheng, G. Wang, X. Quan, Recent Work on Boiling and Condensation in Microchannels, Journal of Heat Transfer, 131(4) (2009) 043211-043215.

[30] G. Wang, P. Cheng, An experimental study of flow boiling instability in a single microchannel, International Communications in Heat and Mass Transfer, 35(10) (2008) 1229-1234.

[31] G. Wang, P. Cheng, A.E. Bergles, Effects of inlet/outlet configurations on flow boiling instability in parallel microchannels, International Journal of Heat and Mass Transfer, 51(9-10) (2008) 2267-2281.

[32] G.L. Wedekind, B.L. Bhatt, An Experimental and Theoretical Investigation Into Thermally Governed Transient Flow Surges in Two-Phase Condensing Flow, Journal of Heat Transfer, 99(4) (1977) 561-567.

[33] B.L. Bhatt, G.L. Wedekind, A Self-Sustained Oscillatory Flow Phenomenon in Two-Phase Condensing Flow Systems, Journal of Heat Transfer, 102(4) (1980) 694-700.

[34] B.L. Bhatt, G.L. Wedekind, K. Jung, Effects of Two-Phase Pressure Drop on the SelfSustained Oscillatory Instability in Condensing Flows, Journal of Heat Transfer, 111(2) (1989) 538-545.

[35] A.E. Bergles, S.G. Kandlikar, On the Nature of Critical Heat Flux in Microchannels, Journal of Heat Transfer, 127(1) (2005) 101-107.

[36] S. Mitra, A. Narain, R. Naik, S.D. Kulkarni, A quasi one-dimensional method and results for steady annular/stratified shear and gravity driven condensing flows, International Journal of Heat and Mass Transfer, 54(15-16) (2011) 3761-3776.

[37] J. Kurita, M. Kivisalu, S. Mitra, R. Naik, A. Narain, Experimental results on gravity driven fully condensing flows in vertical tubes, their agreement with theory, and their differences with shear driven flows' boundary-condition sensitivities, International Journal of Heat and Mass Transfer, 54(13-14) (2011) 2932-2951.

[38] M.K. Maddi, D.P. Rao, Experimental studies on flow boiling in inclined tubes: In the regions encountered in solar collectors, The Canadian Journal of Chemical Engineering, 73(1) (1995) 73-84. 
[39] G.D. Mandrusiak, V.P. Carey, X. Xu, An Experimental Study of Convective Boiling in a Partially Heated Horizontal Channel With Offset Strip Fins, Journal of Heat Transfer, 110(1) (1988) 229-236.

[40] A. Ma, J. Wei, M. Yuan, J. Fang, Enhanced flow boiling heat transfer of FC-72 on micro-pinfinned surfaces, International Journal of Heat and Mass Transfer, 52(13-14) (2009) 2925-2931. [41] K.N. Rainey, G. Li, S.M. You, Flow Boiling Heat Transfer From Plain and Microporous Coated Surfaces in Subcooled FC-72, Journal of Heat Transfer, 123(5) (2001) 918-925. [42] W. Nakayama, T. Daikoku, H. Kuwahara, T. Nakajima, Dynamic Model of Enhanced Boiling Heat Transfer on Porous Surfaces -Part I: Experimental Investigation, Journal of Heat Transfer, 102(3) (1980) 445-450.

[43] W. Hailei, R.B. Peterson, Enhanced Boiling Heat Transfer in Parallel Microchannels With Diffusion Brazed Wire Mesh, Components and Packaging Technologies, IEEE Transactions on, 33(4) (2010) 784-793.

[44] B. Holland, N. Ozman, R.A. Wirtz, Flow boiling of FC-72 from a screen laminate extended surface matrix, Microelectronics Journal, 39(7) (2008) 1001-1007.

[45] D. Liu, S.V. Garimella, Flow Boiling Heat Transfer in Microchannels, Journal of Heat Transfer, 129(10) (2007) 1321-1332.

[46] L. Zhang, E.N. Wang, J.M. Koo, L. Jiang, K.E. Goodson, J.G. Santiago, T.W. Kenny, Enhanced Nucleate Boiling in Microchannels, in: Proc. Micro Electro Mechanical Systems, The Fifteenth IEEE International Conference on, (2002) 89-92.

[47] K. Chih-Jung, A. Kosar, Y. Peles, S. Virost, C. Mishra, M.K. Jensen, Bubble Dynamics During Boiling in Enhanced Surface Microchannels, Microelectromechanical Systems, Journal of, 15(6) (2006) 1514-1527.

[48] C.J. Kuo, Y. Peles, Flow Boiling Instabilities in Microchannels and Means for Mitigation by Reentrant Cavities, Journal of Heat Transfer, 130(7) (2008) 072402-072410.

[49] A. Kosar, C.-J. Kuo, Y. Peles, Reduced Pressure Boiling Heat Transfer in Rectangular Microchannels With Interconnected Reentrant Cavities, Journal of Heat Transfer, 127(10) (2005) 1106-1114.

[50] S. Chandra, C.T. Avedisian, On the Collision of a Droplet with a Solid Surface, in: Proceedings: Mathematical and Physical Sciences (1991), pp. 13-41.

[51] R.N. Wenzel, Surface Roughness and Contact Angle, The Journal of Physical and Colloid Chemistry, 53(9) (1948) 1466-1467.

[52] A.B.D. Cassie, S. Baxter, Wettability of porous surfaces, Transactions of the Faraday Society, 40(0) (1944) 546-551. 
[53] A. Marmur, Wetting on Hydrophobic Rough Surfaces: To Be Heterogeneous or Not To Be?, Langmuir, 19(20) (2003) 8343-8348.

[54] A.M. Peters, C. Pirat, M. Sbragaglia, B.M. Borkent, M. Wessling, D. Lohse,

R. G.H. Lammertink, Cassie-Baxter to Wenzel state wetting transition: Scaling of the front velocity, European Physical Journal E: Soft Matter and Biological Physics, 29(4) (2009) 391-397.

[55] Z. Yao, Y.-W. Lu, S.G. Kandlikar, Direct growth of copper nanowires on a substrate for boiling applications, in: Micro \& Nano Letters, Institution of Engineering and Technology, 6(7) (2011) 563-566.

[56] S. Ujereh, T. Fisher, I. Mudawar, Effects of carbon nanotube arrays on nucleate pool boiling, International Journal of Heat and Mass Transfer, 50(19-20) (2007) 4023-4038.

[57] S. Launay, A.G. Fedorov, Y. Joshi, A. Cao, P.M. Ajayan, Hybrid micro-nano structured thermal interfaces for pool boiling heat transfer enhancement, Microelectronics Journal, 37(11) (2006) 1158-1164.

[58] V. Khanikar, I. Mudawar, T.S. Fisher, Flow Boiling in a Micro-Channel Coated With Carbon Nanotubes, Components and Packaging Technologies, IEEE Transactions on, 32(3) (2009) 639-649.

[59] S.J. Kim, I.C. Bang, J. Buongiorno, L.W. Hu, Surface wettability change during pool boiling of nanofluids and its effect on critical heat flux, International Journal of Heat and Mass Transfer, 50(19-20) (2007) 4105-4116.

[60] J.B. Boreyko, C.-H. Chen, Self-Propelled Dropwise Condensate on Superhydrophobic Surfaces, Physical Review Letters, 103(18), 184501 (2009).

[61] J.B. Boreyko, C.-H. Chen, Self-propelled jumping drops on superhydrophobic surfaces, Physics of Fluids, 22(9), 091110 (2010).

[62] P.C. Stephan, C.A. Busse, Analysis of the heat transfer coefficient of grooved heat pipe evaporator walls, International Journal of Heat and Mass Transfer, 35(2) (1992) 383-391.

[63] C. Kunkelmann, K. Ibrahem, N. Schweizer, S. Herbert, P. Stephan, T. Gambaryan-Roisman, The effect of three-phase contact line speed on local evaporative heat transfer: Experimental and numerical investigations, International Journal of Heat and Mass Transfer, 55(7-8) (2012) 1896-1904.

[64] V.K. Dhir, Mechanistic Prediction of Nucleate Boiling Heat Transfer - Achievable or a Hopeless Task?, Journal of Heat Transfer, 128(1) (2006) 1-12.

[65] J. Kim, Review of nucleate pool boiling bubble heat transfer mechanisms, International Journal of Multiphase Flow, 35(12) (2009) 1067-1076.

[66] S.C. Maroo, J.N. Chung, Nanoscale liquid-vapor phase-change physics in nonevaporating region at the three-phase contact line, Journal of Applied Physics, 106(6) (2009) 064911-064917. 
[67] A.P. Wemhoff, V.P. Carey, Molecular Dynamics Exploration of Thin Liquid Films on Solid Surfaces. 1. Monatomic Fluid Films, Microscale Thermophysical Engineering, 9(4) (2005) 331-349.

[68] J.G. Truong, J.P.C. Wayner, Effects of capillary and van der Waals dispersion forces on the equilibrium profile of a wetting liquid: Theory and experiment, The Journal of Chemical Physics, $87(7)$ (1987) 4180-4188.

[69] J.M. Delhaye, Jump conditions and entropy sources in two-phase systems. Local instant formulation, International Journal of Multiphase Flow, 1(3) (1974) 395-409.

[70] A. Faghri, Heat Pipe Science and Technology, Taylor and Francis, Washington D. C., 1995.

[71] A. Faghri, Transport phenomena in multiphase systems., Elsevier Academic Press., 2006.

[72] E. Begg, D. Khrustalev, A. Faghri, Complete Condensation of Forced Convection Two-Phase Flow in a Miniature Tube, Journal of Heat Transfer, 121(4) (1999) 904-915.

[73] A. Mukherjee, V.K. Dhir, Study of Lateral Merger of Vapor Bubbles During Nucleate Pool Boiling, Journal of Heat Transfer, 126(6) (2004) 1023-1039.

[74] A. Mukherjee, S. Kandlikar, Numerical simulation of growth of a vapor bubble during flow boiling of water in a microchannel, Microfluid Nanofluid, 1(2) (2005) 137-145.

[75] X.Q. Xing, Y.J. Lee, Y.Y. Hoe, X. Zhang, S. Gao, D. Pinjala, A simplified approach to simulate bubble nucleation in flow boiling, in: IEEE 13th Electronics Packaging Technology Conference (EPTC) Proceedings, (2011) 525-529.

[76] A. Narain, Q. Liang, G. Yu, X. Wang, Direct Computational Simulations for Internal Condensing Flows and Results on Attainability/Stability of Steady Solutions, Their Intrinsic Waviness, and Their Noise Sensitivity, Journal of Applied Mechanics, 71(1) (2004) 69-88. [77] L. Phan, A. Narain, Nonlinear Stability of the Classical Nusselt Problem of Film Condensation and Wave Effects, Journal of Applied Mechanics, 74(2) (2007) 279-290.

[78] L. Phan, X. Wang, A. Narain, Effects of exit-condition, gravity, and surface-tension on stability and noise-sensitivity issues for steady condensing flows inside tubes and channels, International Journal of Heat and Mass Transfer, 49(13-14) (2006) 2058-2076.

[79] S.D. Kulkarni, A. Narain, M.T. Kivisalu, S. Mitra, M.M. Hasan, Condenser performance, Control, and Heat Transfer Enhancement Issues Resulting from Elliptic-Sensitivity of Shear Internal Condensing Flows, International Journal of Transport Phenomena, 13(1) (2012) 15-57. [80] Q. Liang, X. Wang, A. Narain, Effects of Gravity, Shear and Surface Tension in Internal Condensing Flows: Results From Direct Computational Simulations, Journal of Heat Transfer, 126(5) (2004) 676-686.

[81] S.G. Kandlikar, A General Correlation for Saturated Two-Phase Flow Boiling Heat Transfer Inside Horizontal and Vertical Tubes, Journal of Heat Transfer, 112(1) (1990) 219-228. 
[82] S.G. Kandlikar, H. Narai, M. Shoji, V.K. Dhir, Handbook of Phase Change: Boiling and Condensation, Taylor and Francis, New York, 1999.

[83] F.P. Incropera, Fundamentals of heat and mass transfer, 6th ed., John Wiley, Hoboken, NJ, 2007.

[84] W. Nusselt, Die Oberflächenkondesation des Wasserdampfes, Z. Ver. Dt. Ing. , 60(27) (1916) 541-546.

[85] E.M. Sparrow, J.L. Gregg, A Boundary Layer Treatment of Laminar Film Condensation, ASME Journal of. Heat Transfer, 81 (1959) 13-18.

[86] J.C.Y. Koh, Film condensation in a forced-convection boundary-layer flow, International Journal of Heat and Mass Transfer, 5(10) (1962) 941-954.

[87] W.M. Rohsenow, Heat Transfer and Temperature Distribution in Laminar Film Condensation, Transactions ASME, 78 (1956) 1645-1648.

[88] M.M. Chen, An Analytical Study of Laminar Film Condensation: Part 1- Flat Plates, Journal of Heat Transfer, 83(1) (1961) 48-54.

[89] V. Dhir, J. Lienhard, Laminar Film Condensation on Plane and Axisymmetric Bodies in Nonuniform Gravity, Journal of Heat Transfer, 93(1) (1971) 97-100.

[90] F. Testu, U. Haruo, Laminar filmwise condensation on a vertical surface, International Journal of Heat and Mass Transfer, 15(2) (1972) 217-233.

[91] D.A. Labuntsov, Heat Transfer in Film Condensation of Pure Steam on Vertical Surfaces and Horizontal Tubes, Teploenergetica, 4 (1957).

[92] S.S. Kutateladze, Fundamentals of heat transfer, Academic Press, New York, 1963.

[93] R. Gregorig, J. Kern, K. Turek, Improved correlation of film condensation data based on a more rigorous application of similarity parameters, Heat and Mass Transfer, 7(1) (1974) 1-13.

[94] F.G. Carpenter, A.P. Colburn, The Effect of Vapor Velocity on Condensation Inside Tubes, Proceedings of the General Discussion of Heat Transfer ASME, (1951) 20-26.

[95] A. Cavallini, R. Zecchin, High Velocity Condensation of R-11 Vapors Inside Vertical Tubes, in: Studies on Heat Transfer in Refrigeration, Proc. International Institute of Refrigeration, Trondheim, Norway, 1971, pp. 385-396.

[96] M.M. Shah, A general correlation for heat transfer during film condensation inside pipes, International Journal of Heat and Mass Transfer, 22(4) (1979) 547-556.

[97] M.K. Dobson, J.C. Chato, Condensation in Smooth Horizontal Tubes, Journal of Heat Transfer, 120(1) (1998) 193-213.

[98] D.W. Shao, E.G. Granryd, Flow pattern, heat transfer and pressure drop in flow condensation part I: Pure and azeotropic refrigerants, HVAC\&R Res., 6(2) (2000) 175-195. 
[99] S. Garimella, J.D. Killion, J.W. Coleman, An Experimentally Validated Model for Two-Phase Pressure Drop in the Intermittent Flow Regime for Circular Microchannels, Journal of Fluids Engineering, 124(1) (2002) 205-214.

[100] C.W. Roh, M.S. Kim, Enhancement of heat pump performance by pulsation of refrigerant flow using a solenoid-driven control valve, International Journal of Refrigeration, 35 (2012) 1547-1557.

[101] A. Narain, Modeling of Interfacial Shear for Gas Liquid Flows in Annular Film Condensation, Journal of Applied Mechanics, 63 (1996) 529-538.

[102] A. Narain, G. Yu, and Q. Liu, Interfacial shear models and their required asymptotic form for annular/stratified film condensing flows in inclined channels and vertical pipes, International Journal of Heat and Mass Transfer, 40(15) (1997) 3559-3575.

[103] A. Narain, J.H. Kurita, M. Kivisalu, A. Siemionko, S. Kulkarni, T.W. Ng, N. Kim, L. Phan, Internal condensing flows inside a vertical pipe: experimental/computational investigations of the effects of specified and unspecified (free) conditions at exit, Journal of Heat Transfer, 129(10) (2007) 1352-1372.

[104] S. Kulkarni, Computational study of internal and external condensing flows and experimental synthesis to investigate their attainability and stability in ground-based and spacebased environments, Ph.D. Dissertation, Michigan Technological University, Houghton, 2010.

[105] M. Kivisalu, N. Gorgitrattanagul, S. Mitra, R. Naik, A. Narain, Shear/pressure driven internal condensing flows and their sensitivity to inlet pressure fluctuations, in: Proceedings of the ASME 2011 International Mechanical Engineering Congress and Exposition, Denver, Colorado, (2011). [106] S.A. Mitra, Development of one-dimensional and two-dimensional computational tools that simulate steady internal condensing flows in terrestrial and zero-gravity environments, Ph.D. Dissertation, Michigan Technological University, Houghton, 2012.

[107] Qing Lu, An experimental investigation of heat transfer with condensation in a horizontal rectangular duct, Ph.D. Dissertation, Michigan Technological University, Houghton, 1992.

[108] Q. Lu, N.V. Suryanarayana, Condensation of a vapor flowing inside a horizontal rectangular duct, 117(2) (1995) 418-424.

[109] M. Kivisalu, A. Narain, J. Kurita, An introduction to $\mathrm{mm}$ scale low kinematic viscosity horizontal channel flow morphology in condensation and pool condensation in the presence of vapor flow (abstract only), in: Proceedings of the $62^{\text {nd }}$ Annual Meeting of the APS Division of Fluid Dynamics, 54(19) (2009) BAPS.2009.DFD.HK.4.

[110] J.H. Kurita, Experimental results on gravity driven fully condensing flows in vertical tubes, their agreement with theory, and their differences with shear driven flows' boundary-condition sensitivities, Ph.D. Dissertation, Michigan Technological University, Houghton, 2011. 
[111] P. Gorgitrattanagul, The length of the annular regime for condensing flows inside a horizontal channel - the experimental determination of its values and its trends, M.S. Thesis, Michigan Technological University, Houghton, 2011.

[112] J.H. Kurita, Experimental investigation of fully condensing downward vapor flows in a vertical tube - unspecified (free) exit condition cases, M.S. Thesis, Michigan Technological University, Houghton, 2007. 


\section{I nnovative partial condensing and boiling flow experiments ${ }^{1}$}

The text of this chapter has been edited from its originally published version [1]. New figures have been added, and some existing figures have been edited for content and/or re-formatted to fit the structure of this dissertation. These changes reflect information that was not available at the time of the original publication and material that was available but was left out of the originally published version due to space constraints.

\section{Abstract: chapter 2}

This paper defines experimental discoveries by Prof. Amitabh Narain's research group regarding the nature of high heat-flux innovative boiler and condenser operations that keeps these devices functional for many critically needed thermal management applications where gravitational force is negligible (naturally or made to be so) relative to other forces (viscous, pressure, etc.). These applications include heat removal from narrow spaces to address high power density electronic cooling needs (i.e. mm-scale boilers and condensers with $\mu \mathrm{m}$-scale flows), gravity insensitive aircraft operations, and zero-gravity applications. Further, the paper presents fundamental condensing and boiling flow results that describe the physics that needs to be utilized to achieve significantly enhanced heat-flux values within the context of these applications.

Reported experimental results demonstrate:

(i) the ability to replace hydrodynamically and thermally ineffective and problematic liquid-vapor configurations (plug/slug, etc.) with annular flows where a flowing thin liquid film covers the entire heat-exchange surface,

1 Large parts of this chapter are taken from: M.T. Kivisalu, P. Gorgitrattanagul, A. Narain, Results for high heat-flux flow realizations in innovative operations of milli-meter scale condensers and boilers, International Journal of Heat and Mass Transfer, 75 (2014) 381-398. It is listed as [1] in the list of references. However, parts of the version reported here have been modified/improved since their original publication as described in the preface and summarized in the first paragraph of this chapter. These and any other changes are not endorsed by the publisher. Permission to reproduce [1] has been granted by the publisher, Elsevier Limited. The license agreement is available upon request from the author, who may be contacted via email at mtkivisa@mtu.edu. 
(ii) the ability to use pulsation-induced interfacial wave phenomena in conjunction with sub-micron thin film phenomena (such as contact line physics) to achieve very high heat-flux during boiling and condensation, and

(iii) the ability to desensitize these flows that would otherwise be highly sensitive to inadvertent but ever present flow fluctuations (due to sensitive coupling between vapor and liquid motions, as well as sensitivity of these micro-meter scale thick liquid films to minuscule transverse vibrations of the heat-exchange surface) when flow channel hydraulic diameters reduce to milli-meter or sub milli-meter scales.

Innovative realizations of shear-driven boiling and condensing flows have been investigated within horizontal channels (with condensation or boiling on the bottom, horizontal surface). Results from boiling and condensing flows of FC-72 fluid in a horizontal rectangular cross-section (2 or $6 \mathrm{~mm}$ gap height and 15 or $24 \mathrm{~mm}$ width, respectively) duct of $1 \mathrm{~m}$ length are presented. Utilizing a controlled presence of mass flow rate fluctuations, additional enhancements (sometimes $>863 \%$ for condensing flows and > $198 \%$ for boiling flows) in heat-transfer rates are obtained for these innovative devices at a location within the device length. This paper reports representative time-varying heat-flux values at this location. Theory based on the nature of these time-varying heat-flux values coupled with other experimental observations reported here support the understanding that this phenomena can potentially be used (in future experiments) to significantly enhance average heat-flux values over the entire length of an innovative boiler/condenser.

The reported phenomena arises from superposing relatively fast time-scale $(2.5-30 \mathrm{~Hz})$ flow rate pulsations on otherwise steady-in-the-mean flows to beneficially change certain flow variable averages - over the longer time-scale $(<0.01 \mathrm{~Hz})$ of practical interest relative to their values obtained in the absence of externally imposed pulsations.

With the right exit flow configuration in the device, such externally imposed pulsations may cause standing waves to form on the thin film interface. The observed asymmetric 
reduction in the mean film thickness (associated with high heat-flux values) is likely due to "stickiness" of wave-troughs resulting from interaction of phenomena associated with three physically distinct length scales (namely nano-scale, micro-scale, and continuum length scales) and two different time scales — short (less than $0.5 \mathrm{~s}$ ) and long (greater than $1 \mathrm{~min})$. That is, associated micro-scale flows at wave-troughs interact with liquid-vapor interface phenomena and, possibly concurrently, de-stabilize the local adsorbed layer (whose thickness may be $<10 \mathrm{~nm}$ and is exposed to phenomena such as disjoining pressures) on the wetting heat-exchange surface.

\subsection{I ntroduction and nomenclature}

This chapter presents some new fundamental results from experimental investigations of shear/pressure-driven internal flow boiling and flow condensation. The study is both comparative and unifying in nature. Traditional condenser (all vapor flow at inlet and all liquid flow at exit) and boiler (all liquid flow at inlet and all vapor flow at exit) operations are shown in Figs. 2.1a and 2.2a respectively. Operations for realizing the innovative condensing and boiling flow conditions are schematically represented in Fig. 2.1b and Fig. 2.2b. 



\section{Traditional Condenser}

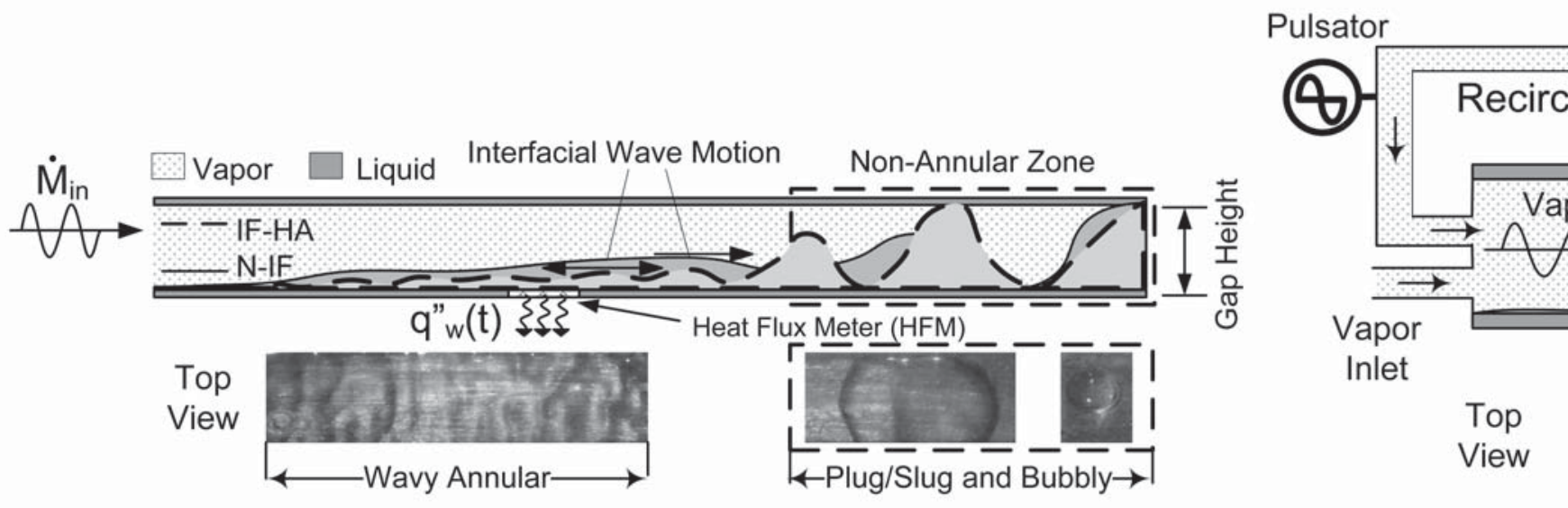

(a)

Figure 2.1. Side-view schematic and representative top-view photographs for (a) traditional and (b) innovative cor innovative condenser, allowing the desired condensation rate with an exit vapor quality less than 1 , even if the net the sense that it does not participate in the condensation process. The very low pressure drop ( < $160 \mathrm{~Pa}$ for the to be reasonably low as well. Videos 2-1 and 2-2 (see accompanying data disc) are representative of the innovativ

\section{Traditional Boiler}
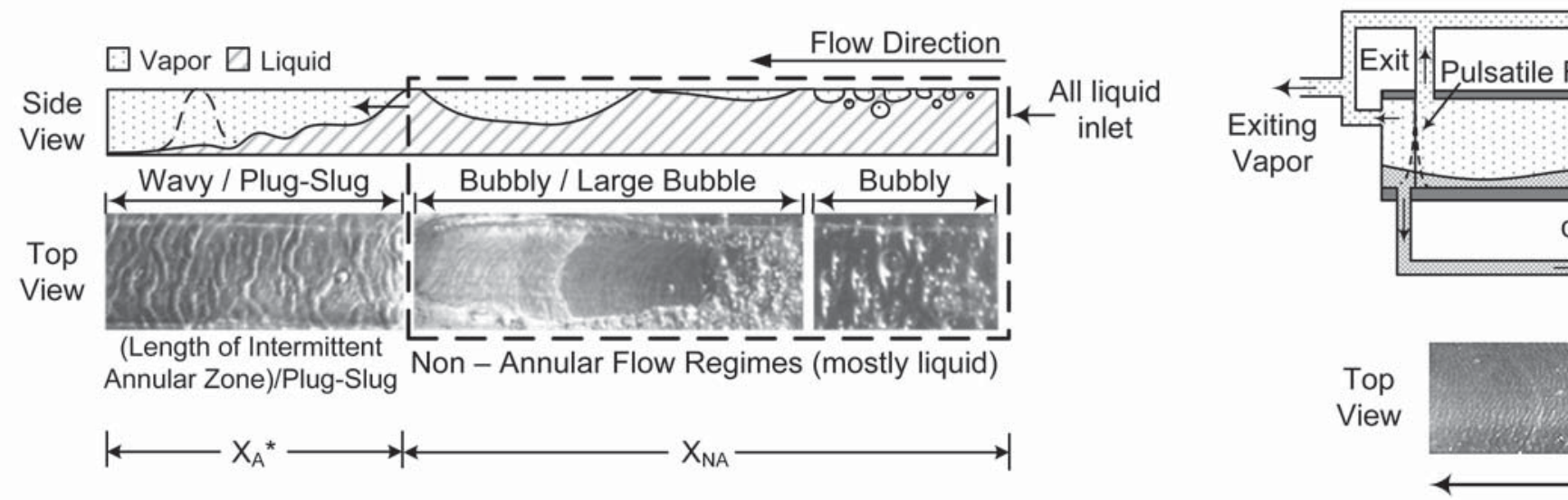

(a)

Figure 2.2. Side-view schematic and representative top-view photographs for (a) traditional and (b) innovative boi innovative boiler, allowing the desired boiling rate with an inlet vapor quality less than 1 . This re-circulating vapor $\mathrm{fl}$ for the experiments reported here) of the annular flow regime allows the pumping power required for the re-circula innovative boiler of (b) without and with imposed fluctuations, respectively. 

The rationale for using re-circulating vapor flows in the innovative arrangements of Fig. 2.1b and Fig. 2.2b is to arrange an appropriate through-flow of vapor that does not undergo phase-change in the condenser or in the boiler.

The innovative boiler operation also has a desired liquid flow rate at the inlet (which may be held at close to saturation temperature) — with or without a left-over re-circulating liquid flow rate at the exit. A left-over re-circulating liquid flow rate at the exit of the boiler is preferred if the boiler is heated by a specified heat-load (as in cooling of electrical or electronic devices) — as it is desirable to avoid critical heat-flux phenomena associated with dry-out conditions. As seen from the representative flow visualization photographs in Figs. 2.1 and 2.2, the innovative arrangements allow effective control of liquid-vapor morphology — restricting them to the efficient annular regime while avoiding complex non-annular condensing and boiling flow regimes (nucleate, bubbly, plug/slug, dry out and critical heat-flux related film boiling regions, etc.) discussed in $[2-11]$.

For horizontal flow boilers of larger than mm-scale hydraulic diameter, nucleate boiling may persist within the annular film and such nucleation could even be beneficial for increasing heat-transfer rates. This paper's focus, however, is to restrict itself to $\mathrm{mm}$ and sub-mm scale flow boilers with micro-meter scale liquid film thicknesses (of interest to applications which require heat removal from small areas or relate to space-based or aircraft-based thermal management), where nucleation, growth, and detachment of bubbles are suppressed to varying degrees — depending on flow conditions and location within the boiler. Ways of suppressing undesired bubble nucleation and growth within most of the annular regime are suggested for these flows. This suppression is observed here and is known [12] to occur when liquid film thickness values are small (film Reynolds numbers < 200) and inlet liquid temperatures are close to saturation temperature. Since thermal resistance to heat-flow through nucleating bubbles is much higher than for direct heat flow through a thin film on a wetting surface, the bubbles do not grow enough to experience buoyancy aided detachment mechanisms before they are 
broken up or removed by the shear stresses within the moving liquid film.

The experiments reported in this paper also introduce pulsations in the incoming FC-72 vapor flow rate (and, for the case in Fig. 2.2b, also in the incoming FC-72 liquid mass flow rate) towards further improving these efficient and innovative annular flows by dramatically enhancing mean heat-flux values (> $100-1000 \%)$ at one or more locations. This is to meet modern challenges where a boiler (using water or a higher latent heat working fluid instead of FC-72 used here) of a given small hydraulic diameter is expected to carry heat-flux values in excess of $1 \mathrm{~kW} / \mathrm{cm}^{2}$ and, concurrently, one needs to reduce overall device-level consumption of pumping power per unit device length (in $\mathrm{W} / \mathrm{m}$ ) and per unit heat-exchange rate (in W). In addition, for many electronic cooling needs, there are added restrictions. They are:

(i) boiling-surface temperature should not exceed about $80^{\circ} \mathrm{C}$,

(ii) the liquid flow into the boiler should not be significantly sub-cooled,

(iii) the condenser coolant should eventually reject heat to ambient air at $20-30{ }^{\circ} \mathrm{C}$, and (iv) other restrictions on device and system level absolute pressures, etc.

Note that earlier experiments [13 - 14] (see also section 3) have established that, in the annular portion of a traditional shear/pressure-driven condenser that operates in a fully condensing mode, the interfacial waves arising from externally imposed mass flow rate pulsations at the inlet significantly change the key mean values of film thickness, heat-flux, etc., relative to realizations in the absence of imposed pulsations. At a location within the annular region, very significant heat-flux enhancements (> $400 \%)$ were reported [13 - 14] but it was unclear as to whether such enhancements could be obtained for the proposed innovative operations (i.e. with re-circulating vapor flows) of condensers and boilers.

Though the literature in boiling (both pool and flow boiling [2 - 11]) and condensation [15 - 18] are quite vast, this paper does not focus on consideration of techniques used for identifying different flow regimes $[2-4]$ and estimating heat-transfer coefficients 
[2-4] associated with the respective regimes. Instead, in the absence of pulsations, we have verified (though not reported here for brevity) that the reported values of experimentally measured heat-flux are in good agreement with new computational techniques - both one-dimensional engineering approaches for annular flow condensation [19] and flow boiling [20], as well as a sophisticated full steady/unsteady computational simulation technique [21 - 24] for annular condensation.

The experiments reported here investigate pulsatile and non-pulsatile annular flows of FC-72 in a horizontal test-section whose total length is $1 \mathrm{~m}$. Its top and side walls are transparent. For innovative flow boiling operations, the channel gap height is $2 \mathrm{~mm}$ and its width is $15 \mathrm{~mm}$, and for innovative flow condensation operations, the channel gap height is $6 \mathrm{~mm}$ and its width is $24 \mathrm{~mm}$.

\section{Nomenclature:}

\section{Accent Notations}

$\overline{\mathbf{X}}$ : Time-averaged value of variable ' $\mathrm{X}(\mathrm{t})$ '

$X^{\prime}$ : Fluctuations in variable ' $X(t)$ ' with $X(t) \equiv \bar{X}+X^{\prime}(t)$

$\mathbf{a}_{X \mid Y}(f)$ : Magnitude of the FFT of variable ' $X_{Y}(t)$ ' taken at frequency ' $f$ ', also known as the amplitude of variable ' $X_{Y}(t)$ ' at frequency ' $f$ '

$\mathbf{X}_{a: b}:$ Variable ' $X$ ' of fluid phase ' $b$ ' taken at location ' $a$ ' (see subscripts section below)

\section{Labels}

h: Gap height of flow channel in test-section (instrumented condenser or boiler used in the reported experiments) [mm]

L: Length of flow channel of interest for flow measurements within the test-section [m] FC-72: Working fluid used in experiments, trade name for 3M Corp. perfluorohexane $\left(\mathrm{C}_{6} \mathrm{~F}_{14}\right)$ FlourinertTM electronic liquid.

DPT: Differential Pressure Transducer: measures pressure-differences directly

APT: Absolute Pressure Transducer: used for direct measurement of absolute pressure at a location of interest 
HFM-40: Heat-flux meter used to measure heat-flux directly at the $x=40 \mathrm{~cm}$ location of interest (see variables section below)

$\mathbf{P}_{\mathrm{v}}$ : Vapor Pulsator used to induce fluctuations in vapor pressure and flow rate supplied to the test-section

$\mathbf{P}_{1}, \mathbf{P}_{2}$ : Pumps used to circulate liquid working fluid in experimental flow loop

OM: Orifice-plate flow Meter for estimating instantaneous dynamic flow rate

TEC: Thermoelectric cooler. These are solid state heat pumps which use electrical current to transfer heat in either direction.

L/V separator: Liquid-vapor separator

DC: Direct Current, as of electrical potential and current

PID: Proportional-Integral-Derivative, as in the feedback control method

N-IF: No-Imposed Fluctuation, referring to cases in which the vapor pulsator was not used

L-IF: Low amplitude-Imposed fluctuation

T-IF: Transition amplitude-Imposed fluctuation

H-IF: High amplitude-Imposed Fluctuation

\section{Variables}

f: Frequency $[\mathrm{Hz}]$

$\mathbf{f}_{\mathrm{P}: \mathrm{V}}$ (same as $\boldsymbol{f}_{\boldsymbol{p}}$ ): Primary frequency associated with imposed vapor phase flow pulsations $[\mathrm{Hz}]$

$\mathbf{f}_{\mathrm{P}: \mathrm{L}}:$ Primary frequency associated with imposed liquid phase flow pulsations $[\mathrm{Hz}]$

$\mathbf{x}$ : Distance downstream of the start of the region of interest in the test-section [cm]

m: Mass flow rate $[\mathrm{g} / \mathrm{s}]$

p: Pressure $[\mathrm{kPa}]$

v: Average velocity over a reference area $[\mathrm{m} / \mathrm{s}]$

$\mathbf{v}_{\mathrm{a}: \mathrm{b}}$ : Estimate of the spatial average of the velocity of flow ' $b$ ' over its cross-sectional area at location ' $\mathrm{a}$ ' $[\mathrm{m} / \mathrm{s}]$

T: Temperature $\left[{ }^{\circ} \mathrm{C}\right]$

q": Heat-flux [W/cm2] 
$\Delta \mathrm{p}_{\mathrm{a}-\mathrm{b}}$ : Pressure difference between locations $\mathrm{x}=\mathrm{a}$ and $\mathrm{x}=\mathrm{b}$, each in units of $\mathrm{cm}$ (see subscripts section below) [Pa or kPa]

t: Time [s]

$\Delta \mathbf{T}$ : Temperature-difference between a representative saturation temperature and a representative heat-exchange surface temperature $\left[{ }^{\circ} \mathrm{C}\right]$

\section{Subscripts}

0: At the $x=0 \mathrm{~cm}$ location

10: At the $x=10 \mathrm{~cm}$ location

40: At the $x=40 \mathrm{~cm}$ location

90: At the $x=90 \mathrm{~cm}$ location

I: Associated with the inlet of the test-section or another flow loop component

E: Associated with the exit of the test-section or another flow loop component

R: Associated with re-circulating vapor flow

P: Associated with imposed pulsations or the vapor pulsator

L: Liquid

V: Vapor

AC: Associated with the auxiliary condenser

B: Associated with the pool boiler of Figs. $2.4-2.5$

Sat: Saturation, as of temperature

Avg: Representative of an average value with respect to distance ' $x$ ' within the testsection 


\subsection{Experimental approach}

a)

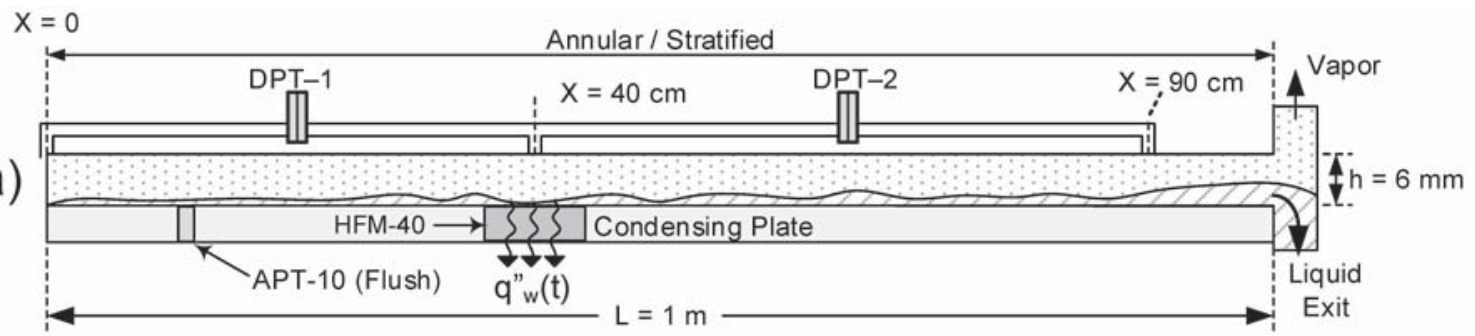

b)

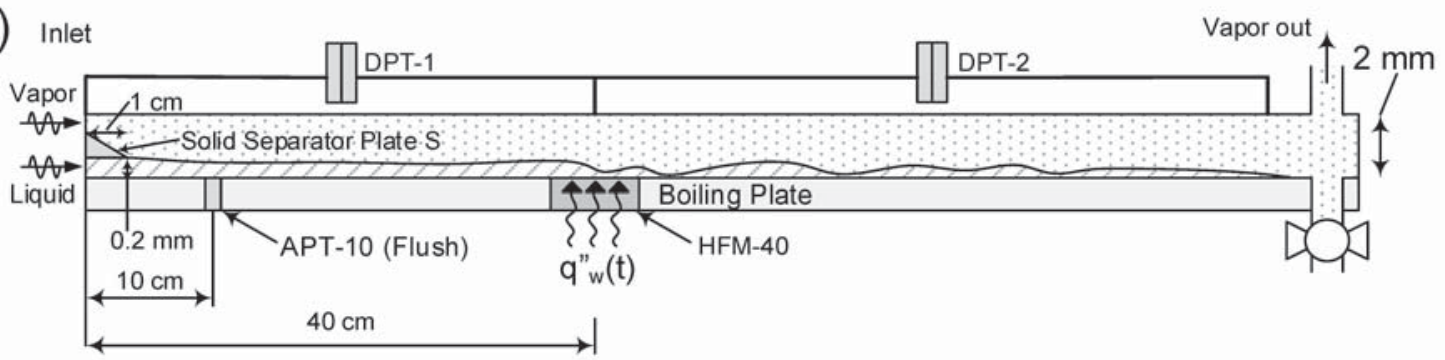

C)

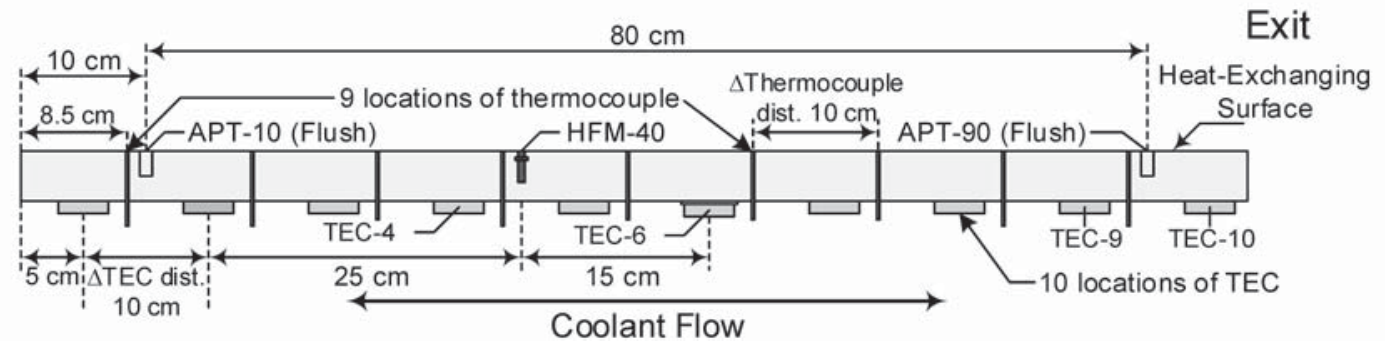

Figure 2.3. Schematics of the test-sections used in the innovative condensing and boiling flow experiments. These schematics represent: (a) the innovative condenser test-section details, (b) the innovative boiler test-section details for a fully boiling case (no liquid removed from exit), and (c) details and instrumentation of the heat-exchange plate ("Condensing Plate" of (a) or "Boiling Plate" of (b)). The solid separator plate ' $S$ ' in (b) was approximately $0.2 \mathrm{~mm}$ thick, leaving a gap of around $1.6 \mathrm{~mm}$ for the inlet vapor flow coming from the re-circulating loop (not shown in (a) - (c)). The 10 TECs in (c) represent thermoelectric modules which may be used to pump heat to or from water-cooled heat sinks (not shown).

\subsubsection{Set-up}

Two horizontal channels were used as shown in Figs. 2.1b and 2.2b. The $6 \mathrm{~mm}$ gap height channel of Fig. 2.3a was used for the innovative condensing flow experiments in the arrangement of Fig. 2.1b. The $2 \mathrm{~mm}$ gap height channel of Fig. $2.3 \mathrm{~b}$ was used for the innovative boiling flow experiments in the arrangement of Fig. 2.2b. The sides and the 
top of these channels are transparent plastic (machined out of transparent polycarbonate plates) covers. Some additional instrumentation details of the condenser and boiler test-sections are given in Figs. 2.3a-c. For both channels, their plastic covers of Figs. 2.3a-b were alternatively mounted on the single horizontal stainless steel plate of Fig. 2.3c, underneath which are 10 thermoelectric modules (TEC) evenly spaced along the 1 meter length of the channel.

These TECs between the plate and 10 individual heat sinks (mounted underneath the TECs in Fig. 2.3c) pump heat to add or withdraw heat from the heat sinks (which are formed by separate arrangements that allow controlled temperature water to flow underneath each of the separate TECs).

For the condensing flow experiments, the thermoelectric modules were not operated, allowing heat to flow by conduction from the condensing-surface to the heat sinks (through the thermoelectric modules) and on to the water flow. For the boiling flow experiments, the thermoelectric modules were used to control boiling-surface temperature and concurrently pump heat into the test-section (through the heat-exchange plate) from the water flows that behave as heat sources (or supply heat sinks).

These thermoelectric modules were operated using a computer-controlled analog variable voltage DC power supply (implemented by means of a $0-20 \mathrm{~mA}$ process current output for each TEC, amplified to $0-10$ amperes by activating the gate of a Darlington type bipolar junction power transistor in series with the TEC, subjected to a fixed 24 volt DC compliance voltage) which was PID controlled to maintain desired wall temperature distributions for the boiling flow runs.

Thus it should be noted that for the boiling flow results reported here, the temperature control of the boiling-surface is automated, yielding approximately constant (in time) boiling surface temperatures. This is a different heating approach than the ones typically used for boiling flow experiments (such as the one used by us in [20] with a completely 
different test-section). Typical heating approach (as in [20]) consists of controlled modular electric heating, yielding known heat load in each heating module. The change in approach here was necessitated by the need:

(i) to impose small values of fluid-to-surface temperature difference $(\Delta T)$ - in the range of $3^{\circ} \mathrm{C}$ to $15^{\circ} \mathrm{C}$ - so as to ensure that the length of complete boiling (for given inlet flow rate conditions) was sufficiently large $(>60 \mathrm{~cm})$ to be well ahead of the heat-flux meter's location (around $x=40 \mathrm{~cm}$ in Fig. 2.3); and

(ii) to avoid critical heat-flux issues in the dry out region (which was unavoidable because of the rather long test-section length of $1 \mathrm{~m}$, contrasted with the $0.7 \mathrm{~m}$ long test-section used in [20]).

Similar automated feedback control of boiling surface temperatures using standard electrical surface heaters was impractical because the same heat exchange plate of Fig. 2.3c was being used for both condensing and boiling flow experiments, the TEC configuration allowed easy and rapid transition between both modes of operation, and the equipment for the TEC system was already available in the laboratory from earlier condensing flow investigations of [13-14] (see also chapter 3).

For each of the two test-section configurations, two differential pressure transducers, DPT-1 and DPT-2 shown in Figs. 2.3a-b, were used to record the vapor pressure-difference over the first $40 \mathrm{~cm}$ of the channel and between $\mathrm{x}=40 \mathrm{~cm}$ and $x=90 \mathrm{~cm}$ along the length of the channel.

Mounted within the plate shown in Fig. 2.3c is a flush heat-flux meter (HFM) whose center is located at the $x=40 \mathrm{~cm}$ location in Fig. 2.3a. For this reason, this heat-flux meter is denoted as HFM-40. The $1 / 4$ inch outer diameter probe has a circular cross-section sensing area with the diameter being approximately $5.55 \mathrm{~mm}$. The probe was also centered across the channel width. The probe is of the thin-film thermopile type (as part of a removable probe supplied by Vatell Corp.) and was able to measure the time-varying heat-flux and surface temperature - over the 5.5 - $5.6 \mathrm{~mm}$ diameter 
circular zone whose center was $40 \mathrm{~cm}$ downstream from the test-section inlet $(x=0$ in Fig. 2.3). Heat flow between the probe and the rest of the heat exchange surface is by conduction with the assistance of thermal paste at the contact surfaces.

Thermocouples mounted in the plate recorded the temperature of the refrigerant (FC-72) along the center of the flow channel at distances marked in Fig. 2.3c. The thermocouples measure within a $1 \mathrm{~mm}$ radius, and their tips are axially located within $\pm 0.5 \mathrm{~mm}$ of the condensing/boiling surface. Two flush pressure transducers, APT-10 and APT-90 shown in Fig. 2.3c, were used to measure the absolute pressures at the $x=10 \mathrm{~cm}\left(p_{10}\right)$ and $x=90 \mathrm{~cm}\left(p_{90}\right)$ locations along the channel. 


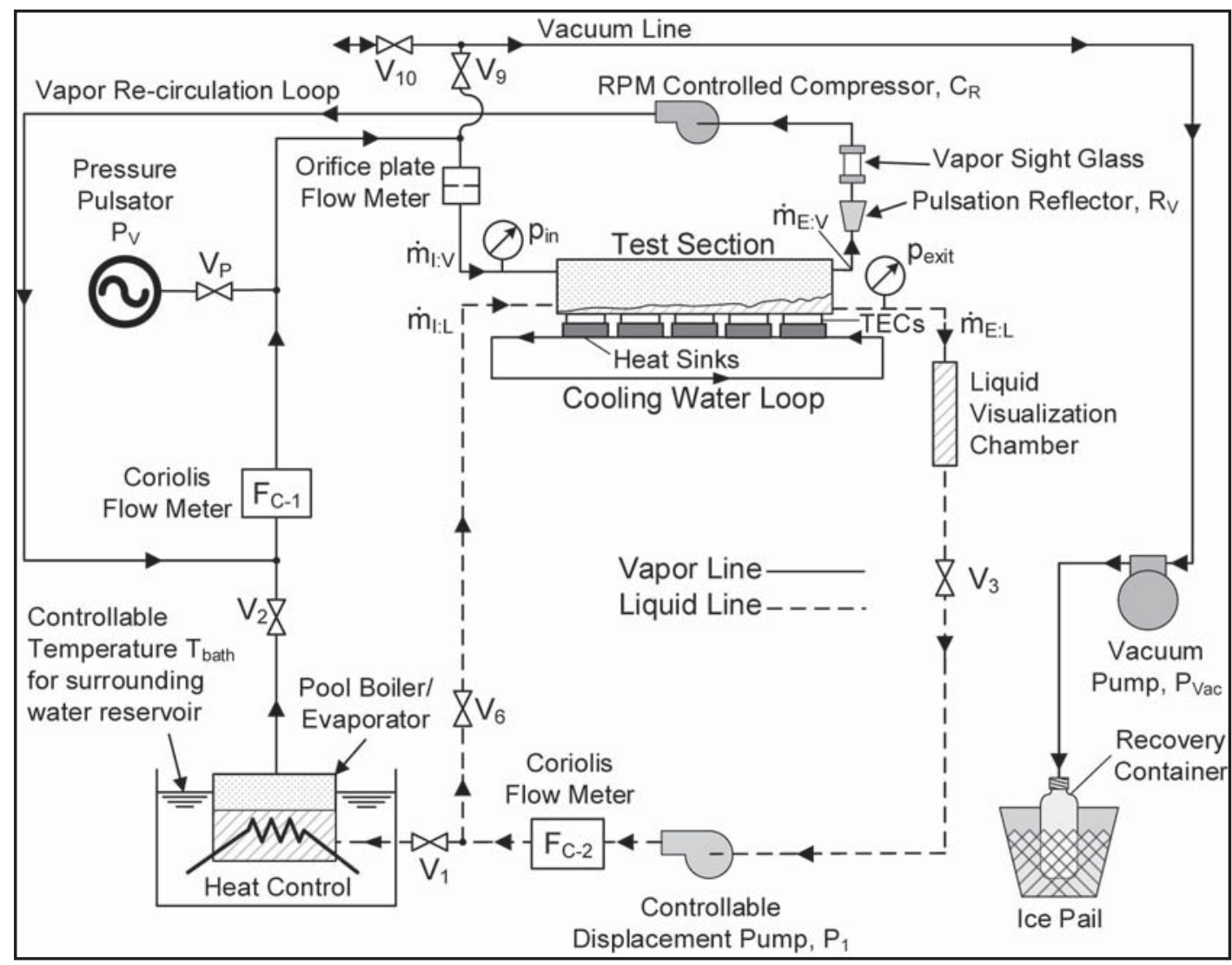

Figure 2.4. System schematic for flow condensation experiments relevant to the innovative design of Fig. 2.1b. For visual clarity, only 5 heat sinks and 5 TECs are shown underneath the test-section; however, there were 10 of each used in the experiments (see Fig. 2.3c for the locations of the 10 TECs). The compressor $C_{R}$ was used to control the mass flow rate of the re-circulating vapor, and the pressure pulsator $P_{V}$ and valve $V_{P}$ were used to control the frequency and amplitude of imposed inlet vapor pulsations, respectively. Displacement pump $P_{1}$ was used to control the test-section pressure by removing the condensed liquid at a feedback-controlled rate, and the pool boiler heat control was used to set the desired mass flow rate of vapor supplied to the test-section by re-boiling the condensed liquid at a feedback-controlled rate. For all experiments reported here, the liquid mass flow rate supplied to the condenser $\dot{m}_{\text {l:L }}$ was zero. 


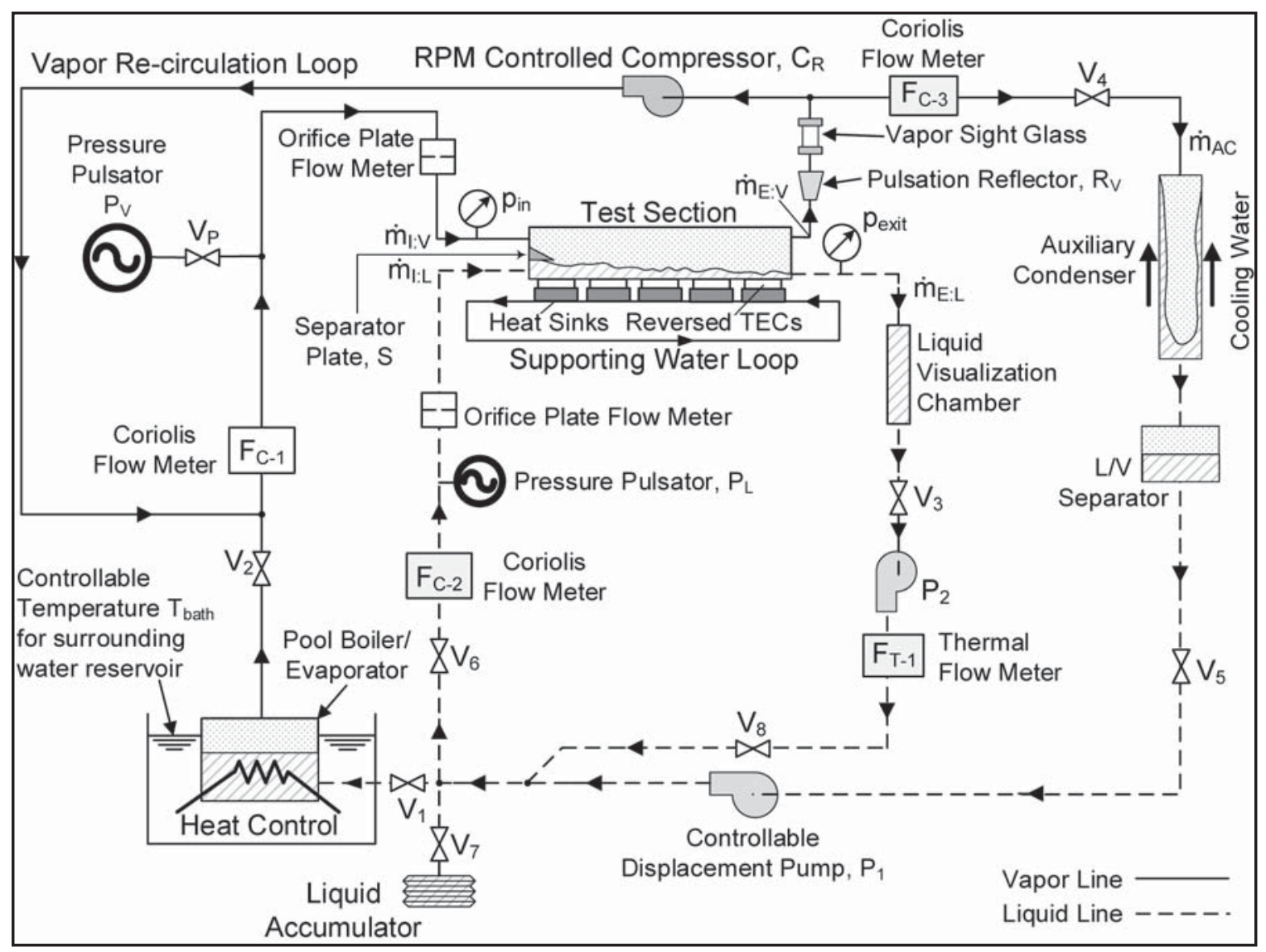

Figure 2.5. System schematic for flow boiling experiments relevant to the innovative design of Fig. 2.2b. For visual clarity, only 5 heat sinks and 5 reversed TECs are shown underneath the test-section; however, there were 10 of each used in the experiments (see Fig. 2.3c for the locations of the 10 TECs). The compressor $C_{R}$ was used to control the mass flow rate of the re-circulating vapor, and the pressure pulsator $P_{V}$ and valve $V_{P}$ were used to control the frequency and amplitude of imposed inlet vapor pulsations, respectively. The liquid boiled within the test-section was condensed in the auxiliary condenser. Displacement pump $P_{1}$ was used to control the test-section pressure by removing the condensed liquid at a feedback-controlled rate, and the pool boiler water temperature $T_{\text {bath }}$ was used to guide the system pressure. For all experiments reported here, the liquid mass flow rate removed from the boiler $\dot{m}_{E: L}$ was zero, so pump $P_{2}$ was not used.

The test-sections of Figs. 2.1b and 2.2b were mounted, respectively, within the flow loops shown in Figs. 2.4 and 2.5. These flow loops provided the re-circulating vapor flow and the vapor or liquid used for phase change. Computer-controlled variable-speed peristaltic pumps $P_{1}$ and $P_{2}$ were used to pump the liquid. 
The re-circulation loop was driven by a semi-hermetically sealed, rotary-vane type compressor $\mathrm{C}_{\mathrm{R}}$, which is driven by a variable-speed external $\mathrm{DC}$ motor using magnetic coupling through the wall of the heated (thermostat controlled) compressor housing. The speed of the motor, which may be manually or computer controlled, was used to adjust the re-circulating vapor flow rate supplied by the compressor.

The test-section heat sinks were connected to a water loop of thermostatically or PID controlled temperature. The flow rate of this water loop (indicated in Figs. 2.4-2.5) was manually controlled through the use of bypass and throttling valves.

A pressure pulsator $P_{v}$, consisting of a variable speed DC powered diaphragm compressor (with the valves removed between its suction and discharge chambers), was used to introduce pressure and flow rate pulsations to the vapor supplied to the test-section. The speed of this pulsator was manually set through its motor controller to set the predominant frequency of the imposed vapor pulsations, and the throttling valve $V_{P}$ was manually adjusted to control the amplitude of the imposed vapor pulsations.

An orifice-plate flow meter "OM" (with measurements of dynamic pressure-difference across the device and dynamic absolute pressure at the inlet or exit of the device) was used to allow estimation of the dynamic mass flow rate fluctuations in the vapor supplied to the test-section according to the method described in [25]. These dynamic mass flow rates are not reported here.

In the vapor line exiting the test-section, a pulsation reflector $R_{v}$ (consisting of a diffuser impinging on a flat plate with a circular pattern of small holes) was used to assist in reflecting acoustic energy — associated with vapor pressure/flow rate pulsations — back into the test-section.

The temperature of the water reservoir surrounding the pool boiler was used to guide the 
system pressure to desired quasi-steady (approximately steady-in-the-mean with respect to time) levels. The system pressure level prior to start-up (approximately measured to be between $120 \mathrm{kPa}$ and $150 \mathrm{kPa}$ at the pool boiler) determines the average system pressure (which could be defined by an average of measured pressures at different locations - such as pool boiler, test-section inlet, etc.) at steady or quasi-steady state flow operations. For the reported experiments, these "system pressures," corresponding to approximately steady flow rates, are adequately represented by the pressure at one location in the flow-loop - such as pressure $p_{10}$ values reported in Tables $2.1-2.3$.

The internal ring-type heater of the pool boiler was computer controlled through a solid-state relay activated by pulse-width modulation (PWM) using a square wave of 0 / 5 volts at a carrier frequency of $1000 \mathrm{~Hz}$. The pool boiler was used to evaporate the liquid condensed in the test-section for the condensing flow experiments. It was also used to assist in fluid inventory control of the loop for both the condensing and boiling flow experiments.

For the boiling flow experiments, a vertical counter-flow heat exchanger (of stainless steel welded construction, with refrigerant flowing downward through a $6.8 \mathrm{~mm}$ I.D. tube in the center and cooling water flowing upward in the annulus) was used to condense the refrigerant boiled in the test-section. It is labeled the Auxiliary Condenser in Fig. 2.5, and its cooling water was part of a closed loop with the shell side of a shell-and-tube type heat exchanger (stainless steel), a centrifugal pump, and an elevated water tank. The water flow through the tube side of the heat exchanger was provided from a separate chilled water loop at a controlled temperature and constant flow rate.

All data was logged and recorded using National Instruments hardware and a desktop computer as described in chapter 3 and [14]. All computer controls and management of data during experiments were implemented through LabVIEW 10.0.1 (32 bit). Additional details of the experimental system, instrumentation, and instrumentation accuracies are available in chapter 3 and [14]. Resulting uncertainties in measured or calculated data 
reported here may be found in the column headers of Tables $2.1-2.2$ and in relevant figure captions.

\subsubsection{Procedures}

Start-up begins with ascertaining the amount of liquid within the system. Valve $V_{10}$ in Fig. 2.4 remains open when the system is not in use, so the vacuum line is at atmospheric

pressure. Purge valve $\mathrm{V}_{9}$ in Fig. 2.4, connected to this vacuum line near the highest point in the flow loop, is opened to expose the flow loop to atmospheric pressure, facilitating the addition or removal of liquid. For clarity, the vacuum system is not shown in Fig. 2.5. Valves $\mathrm{V}_{1}$ and $\mathrm{V}_{6}$ in Figs. 2.4 - 2.5, and valve $\mathrm{V}_{5}$ in Fig. 2.5 (for boiling experiments only), are closed to allow individual sections of the flow loop to be filled separately with liquid FC-72. Valve $\mathrm{V}_{7}$ in Fig. 2.5 remains closed (the "liquid accumulator" of Fig. 2.5 was not used in experiments reported here). All other valves in Figs. 2.4 - 2.5 are opened. Valves $\mathrm{V}_{4}$ and $\mathrm{V}_{5}$, not shown in Fig. 2.4, remain closed throughout start-up and operation of condensing flow experiments.

After the appropriate amount of liquid has been added to the flow loop, vacuum pump $\mathrm{P}_{\mathrm{Vac}}$ in Fig. 2.4 (oil-less diaphragm type, not shown in Fig. 2.5) is started. The vacuum line and flow loop are isolated from atmospheric pressure by closing valve $\mathrm{V}_{10}$ in Fig. 2.4. The vacuum pump is run for $1-4$ hours until fluctuations in the system pressures (due to air and FC-72 vapor bubbles escaping from the liquid) disappear, and vapor pressures in the flow loop are approximately $20-25 \mathrm{kPa}$ absolute, slightly below the saturation pressure of FC-72 at laboratory ambient temperatures of $20-25^{\circ} \mathrm{C}$.

During a portion of the evacuation process, the pressure pulsator $\mathrm{P}_{\mathrm{V}}$ and the compressor $C_{R}$ of Figs. $2.4-2.5$ are run at low speeds to help push accumulated air out into the main part of the flow loop, where it will rise due to buoyancy effects and be eliminated from the flow loop via the vacuum line. Liquid FC-72 is recovered from the discharge tube of the vacuum pump using a re-sealable recovery container cooled by ice in a plastic pail (see Fig. 2.4). The cold walls of the container condense the FC-72 vapor coming out of 
the vacuum pump.

At the end of the evacuation process, vacuum-line valve $V_{9}$ (see Fig. 2.4) is closed. Then valve $V_{10}$ is opened to expose the vacuum line to atmospheric pressure. After running the vacuum pump 15 more minutes to cool its motor, it is turned off. Valves $V_{2}, V_{3}$, and $V_{6}$ in Figs. 2.4 - 2.5, and $\mathrm{V}_{5}$ in Fig. 2.5 (for boiling experiments only), are also closed. The pool boiler water reservoir temperature control is adjusted to a temperature corresponding to the saturation temperature of a target operating pressure for the flow loop.

When the pool boiler water reservoir temperature is near its set point, rope heaters on the vapor lines and surrounding the pulsator head and compressor housing are activated. Their voltages are adjusted to reduce undesired condensation of the FC-72 vapor. At or before this time the cooling water flow through the heat sinks under the test-section is started, its temperature control is adjusted to the desired cooling (or heating, for boiling flow experiments) water temperature, and the TEC controls are turned on and set to the desired temperatures (if TECs are used for cooling/heating). Once the pool boiler water reservoir temperature has reached its set point, system pressures are checked, and the water bath temperature control is adjusted accordingly. Valve $\mathrm{V}_{2}$ is then opened, allowing FC-72 vapor to pressurize the remainder of the flow loop.

Once flow has started from the pool boiler to the test-section, the liquid flow is initiated. This is accomplished differently depending on whether innovative (all-annular) condensing or boiling flows are being investigated.

For the condensing flow experiments (see Fig. 2.4), valves $V_{1}$ and $V_{3}$ are opened, and liquid pump $\mathrm{P}_{1}$ is started at a reasonable fixed pumping rate. The compressor is set at a low speed to prevent backflow due to the test-section pressure-drop. The pool boiler internal heater is set at a manual power level corresponding to the heat required to vaporize at the mass flow rate measured by Coriolis meter $\mathrm{F}_{\mathrm{C}-1}$. Once the test-section pressures begin to stabilize, the compressor speed is increased to achieve the desired 
vapor re-circulation flow rate, and the PID control on pump $P_{1}$ is set to hold the test-section inlet absolute pressure, measured by transducer APT-10 of Fig. 2.3a, to a desired value.

For the boiling flow experiments (see Fig. 2.5), valves $\mathrm{V}_{3}, \mathrm{~V}_{4}, \mathrm{~V}_{5}$, and $\mathrm{V}_{6}$ are opened, while valve $V_{1}$ remains closed. The cooling water pump (not shown in Fig. 2.5) for the auxiliary condenser is activated. Liquid pump $P_{1}$ is started at a fixed pumping rate corresponding to the desired test-section liquid inlet mass flow rate measured by Coriolis meter $F_{C-2}$, but liquid pump $P_{2}$ remains turned off. Because the peristaltic pumps $P_{1}$ and $P_{2}$ are positive displacement pumps with at least one roller engaged at all times, there is no net flow of liquid out of the exit of the test-section when pump $\mathrm{P}_{2}$ is turned off. The pool boiler internal heater remains turned off. The compressor is set at a speed corresponding to the desired inlet vapor flow rate measured by Coriolis meter $\mathrm{F}_{\mathrm{C}-1}$.

For each of the two flow configurations, flow condensation and flow boiling, the first step after purge and start-up procedures for the flow loops of Figs. $2.4-2.5$ is to obtain quasi-steady (approximately steady-in-the-mean) flow of FC-72 fluid, pressure, and temperature conditions in the system without any imposed vapor pulsations.

It is important to note that, even when no vapor pulsations are imposed, there are some turbulence and compressor-vane-induced pressure fluctuations in the vapor, vibrations of the condensing surface (from various equipment in the flow loop and other sources), and liquid flow surges arising from the rollers of the peristaltic pumps. Nevertheless, these liquid flow surges and associated pressure fluctuations (whose signatures are present in some of the dynamic measurements) did not significantly shift the time-averaged dynamic heat-flux values at $x=40 \mathrm{~cm}$ until, as discussed later, vapor flow pulsations were deliberately imposed.

Once the flow in the test-section has been established, vapor flow pulsations are imposed at various amplitude levels and frequencies to investigate their effects on heat-flux at the 
$x=40 \mathrm{~cm}$ location and on the liquid-vapor interface morphology within the test-section. It has been experimentally verified that when vapor pulsations were reduced or removed, one recovered results from lower amplitude or no-imposed fluctuation cases.

\subsubsection{Innovative condensing flow experiments}

The innovative condensing flow experiments are run using the flow loop shown in Fig. 2.4. For these experiments, pump $P_{1}$ removed the liquid condensed within the test-section - after it had passed through the liquid visualization chamber. This liquid was then passed through coriolis meter $\mathrm{F}_{\mathrm{C}-2}$ into the pool boiler through valve $\mathrm{V}_{1}$, where it was boiled and sent up the vapor line to be combined with the re-circulating vapor flow from the discharge line of compressor $\mathrm{C}_{\mathrm{R}}$, before passing through coriolis meter $\mathrm{F}_{\mathrm{C}-1}$ and entering the test-section. Valve $\mathrm{V}_{6}$ was closed for all of the condensation experiments reported here, so all of these condensing flows started from a pure vapor state. At the end of the test-section, the exiting vapor flow from the top of the test-section went through a pulsation reflector $R_{v}$ and a sight glass into the suction line of compressor $C_{R}$.

For these experiments, the temperature (at a specified location) and flow rate of the water flow underneath the heat sinks (which, in turn, are underneath TECs in Fig. 2.3c) of Fig. 2.4 were held fixed. The heat-exchange surface temperatures were allowed to become steady and develop suitable spatial variations along the length of the channel. These variations depend on the thermal resistances of the bottom plate, the TECs, the heat sinks, and the condensing flow. As discussed in [14] and section 3.4.2, the resulting non-dimensional condensing-surface temperature variation is approximately fixed and defines a fixed cooling method.

After start-up, the pool boiler internal heater was switched to PID control, with the process variable being the absolute pressure of the vapor measured at the top of the pool boiler. The setting of this pressure, concurrent with the specification of the test-section inlet absolute pressure, approximately fixed the total mass flow rate (vapor boiled in the pool boiler and re-circulating vapor) supplied to the test-section, as described below. 


\subsection{Non-pulsatile flows resulting from no-imposed fluctuations (N-IF) cases}

This procedure involves:

(i) holding the chilled water temperature and flow rate supplied to the test-section heat sinks at a fixed value - consistent with the desired temperature distribution and mean temperature of the condensing surface,

(ii) holding the compressor at a fixed speed - compatible with the desired re-circulating vapor mass flow rate,

(iii) keeping the temperature of the water bath surrounding the evaporator fixed at a value which is a few degrees Celsius below the saturation temperature (at the evaporator pressure) of the refrigerant,

(iv) using PID control of the evaporator internal heater to hold the evaporator pressure fixed, and

(v) using PID control of the liquid pumping rate through pump $P_{1}$ to fix the test-section pressure at the $\mathrm{X}=10 \mathrm{~cm}$ location.

The combination of (iv) and (v) effectively fixes the vapor flow rate through $\mathrm{F}_{\mathrm{C}-1}$ (this is due to pressure drop constraints within the vapor lines between the evaporator and the test-section), which includes both the vapor flow rate from the pool boiler and the re-circulating vapor mass flow rate, the second of which depends mainly on the compressor speed. Once the test-section inlet pressure and exit liquid mass flow rate have stabilized, the test-section quasi-steady exit liquid mass flow rate becomes equal to the quasi-steady mass flow rate of the vapor exiting the pool boiler through valve $V_{2}$ under the constraints of conservation of mass. Thus the liquid within the pool boiler also comes to a steady-in-the-mean level. Constraints (iii) - (iv) are iteratively adjusted as necessary to ensure that $(a)$ the liquid visualization chamber of Fig. 2.4 is completely full of liquid and $(b)$ the liquid does not "pool" at the exit of the test-section (i.e. it drops off the end of the condensing plate as shown in Fig. 2.3a).

In this way, mass flow rate of the liquid condensed in the test-section seeks its "natural" value subject to the supplied re-circulating vapor flow rate, fixed inlet pressure, and 
fixed cooling condition. The "natural" steady value is self-sought as no exit-condition dependency exists for this case.

\subsection{1. 2. Pulsatile flows resulting from imposed fluctuations (IF) cases}

For these cases, in Fig. 2.4, with all the flow controls retained to be the same as previously stated for N-IF cases, the pulsator $P_{V}$ is run at a fixed speed, and the valve $\mathrm{V}_{\mathrm{P}}$ opening (partially or completely opened) is fixed. Generally, this valve is first fully opened, producing the maximum available amplitude of imposed vapor flow pulsations and resulting pressure fluctuations supplied to the orifice meter and test-section inlet. The relatively high pressure drop within the coriolis flow meter $\mathrm{F}_{\mathrm{C}-1}$ effectively damps out most of the imposed vapor pressure fluctuations in the upstream direction, leaving little or no effect of these fluctuations on the evaporator vapor pressure. If necessary, the evaporator pressure set point is adjusted to obtain the same total inlet vapor mass flow rate as was measured for the corresponding N-IF case, and the compressor speed is adjusted to approximately regain the liquid level in the exit liquid tube estimated from the hydrostatic pressure head at the bottom of the liquid visualization chamber of Fig. 2.4. This level is calculated from the difference between $p_{90}$ and the absolute pressure measured at the bottom of the visualization chamber, with the density taken at the average temperature between the measured exit liquid and room temperatures. Generally, this level remains between the top of the liquid visualization chamber and the exit of the test-section (see Fig. 2.4) as specified for the N-IF case.

\subsubsection{Innovative boiling flow experiments}

The innovative boiling flow experiments are run using the flow loop shown in Fig. 2.5. For these steady-in-the-mean experiments, pump $\mathrm{P}_{1}$ removes liquid from the "L/V separator" at the exit of the auxiliary condenser, and Pump $\mathrm{P}_{2}$ removes liquid from the exit of the test-section (if its flow rate is non-zero). The combined liquid from pumps $\mathrm{P}_{1}$ and $\mathrm{P}_{2}$ is supplied to the test-section inlet via valve $\mathrm{V}_{6}$ (with valves $\mathrm{V}_{1}$ and $\mathrm{V}_{7}$

remaining closed). This liquid, after passing through coriolis meter $\mathrm{F}_{\mathrm{C}-2}$, enters the 
test-section underneath an alloy steel separator plate 'S', which separates it from the vapor flow coming from above and directs the liquid along the boiling surface, where it partially or completely boils. Complete boiling of the liquid supplied to the test-section occurred for all of the cases reported in this paper; therefore, pump $\mathrm{P}_{2}$ was stopped (with zero or negligible $\dot{m}_{E: L}$ ) for all of the cases reported in this paper.

The vapor exiting the test-section passed through the pulsation reflector and vapor sight glass, after which the re-circulating component of the vapor flow was removed by compressor $C_{R}$. The remaining vapor was sent to the auxiliary condenser where it condensed partially or completely. Any vapor exiting the bottom of the auxiliary condenser was condensed within the "L/V separator," thus completing the cycle.

The orifice plate flowmeter and pulsator $P_{L}$ in the liquid line, as well as the liquid accumulator shown in Fig. 2.5, were not used for these experiments. Incomplete boiling cases in the proposed configuration of Fig. $2.2 \mathrm{~b}$ are currently being investigated with the help of thermal flow meter $\mathrm{F}_{\mathrm{T}-1}$ and a shorter flow channel, and results are to be reported in future publications.

Due to safety concerns and hardware limitations associated with flowing hot fluid through the heat sinks of Fig. 2.5, the test-section heat-exchange surface heating for the innovative boiling experiments was accomplished with the assistance of the TECs of Fig. $2.3 \mathrm{c}$ between the plate and the heat sinks of Fig. 2.5 through which water flows at a controlled temperature lower than the desired boiling surface temperature. These TECs were configured with the direction of electric current flow reversed from the innovative condensation experiments ("reversed TECs" of Fig. 2.5), which reversed the direction of heat transfer through the TECs. They were PID controlled using the surface temperature measurements as feedback variables.

Thus boiling flow experiments were also conducted in the fixed and specified surface temperature distribution mode, where the mean (temporally averaged over several 
minutes or longer) wall temperatures did not change significantly from case to case - a different approach from the fixed coolant condition (flow rate and temperature) used in the innovative condensing flow experiments (in which the thermal resistance of the heatexchange surface caused the time-averaged condensing surface temperatures to vary in response to their corresponding time-averaged heat transfer rates).

After start-up, before obtaining boiling cases B1 - B6 in Table 2.3, the pool boiler internal heater was started under PID control of the measured absolute vapor pressure of the pool boiler, the set point of which was adjusted by trial and error to achieve the desired test-section inlet absolute pressure. This control remained active for the duration of cases B1 - B6. However, for boiling cases B7 - B10 in Table 2.3, the pool boiler internal heater remained turned off after startup, and once the desired system pressure had been achieved and was stabilized (with the help of the pool boiler water reservoir temperature control), valve $\mathrm{V}_{2}$ was closed, isolating the pool boiler from the rest of the flow loop.

\subsection{Non-pulsatile flows resulting from no-imposed fluctuations (N-IF) cases}

This procedure involves:

(i) using the bath temperature and internal heater control of the evaporator to achieve the desired system pressure,

(ii) eventually (after purge and start-up procedures have been completed) shutting valve $\mathrm{V}_{2}$ (while valve $\mathrm{V}_{1}$ continues to remain closed) to isolate the evaporator from the system (for boiling cases B1 - B6 in Table 2.3, this step was not used, which helped reduce drifts in system pressure caused by the use of the pulsator),

(iii) holding the water temperature and flow rate supplied to the auxiliary condenser consistent with the desired condenser-surface temperature in the auxiliary condenser (as low as possible, for the cases reported here),

(iv) using PID control of the thermoelectric modules underneath the test-section (Fig. 2.3c) to set the boiling-surface temperature profile, 
(v) holding the compressor $\mathrm{C}_{\mathrm{R}}$ at a fixed speed compatible with the desired re-circulating vapor mass flow rate, and

(vi) manually adjusting the liquid pumping rate through pump $P_{1}$ to set a desired inlet liquid mass flow rate (when valve $\mathrm{V}_{6}$ is open and valve $\mathrm{V}_{1}$ is closed).

For incomplete boiling cases (not reported here), PID control of liquid pump $\mathrm{P}_{2}$ would be used to concurrently maintain the level of refrigerant within the liquid visualization chamber (as calculated using the hydrostatic pressure method mentioned in section 2.2.2.1.2) under operating conditions such that there is continuous liquid flow at the exit of the test-section.

The length required to completely boil the liquid in the test-section seeks its "natural" value subject to the supplied liquid flow rate, re-circulating vapor flow rate, inlet pressure (set by the fluid inventory control of the evaporator during step (i)), and fixed boiling-surface temperature conditions (which set the amount of boiling-surface superheat). All the liquid boiled within the length of the test-section for all of the cases reported here, and a thin liquid film was observed up to the last few centimeters of the test-section. This indicates that the required length to completely boil the liquid supplied to the test-section under the chosen operating conditions was slightly less than the test-section length.

\subsubsection{Pulsatile flows resulting from imposed fluctuations (IF) cases}

For these cases, with all the flow controls retained to be the same as previously stated for $\mathrm{N}$-IF cases, the pulsator $\mathrm{P}_{\mathrm{V}}$ is run at a fixed speed, and the valve $\mathrm{V}_{\mathrm{P}}$ is partially or completely opened. The chosen pulsator frequency for the vapor flow is set at the frequency associated with incoming liquid pulsations (determined by the steady rpm of pump $P_{1}$ ) for all the runs reported here (see Table 2.3), or at other values (not reported here). For the cases reported here, the pulsator speed and setting of valve $V_{P}$ opening were the only changes made to the system in going from N-IF to different imposed vapor fluctuation cases. 


\subsubsection{Data processing}

The basic features of collected data are reported in Tables $2.1-2.3$. The experimental runs are characterized by case number. The letter ' $\mathrm{C}$ ' precedes a number to designate an innovative condensing flow case, whereas the letter ' $\mathrm{B}$ ' precedes a number to designate an innovative flow boiling case. Each case represents a unique set of experimental conditions which were held for a period of time ranging from $20-77$ minutes. From this data, segments of 20 - 40 minutes were selected for averaging of the relevant flow variables acquired at the low DAQ rate $(\sim 1.0 \mathrm{~Hz})$. Ten sets of dynamic data (11 for case C8) at $2000 \mathrm{~Hz}$ DAQ rate and 10000 sample record length (each of a 5 second duration) were chosen for obtaining and reporting (in Tables $2.2-2.3$ ) the representative amplitude of different variables for each of the run cases. Due to programming limitations, 6 second gaps were created in the time history of the low DAQ rate flow variable data each time a dynamic data set was acquired.

Since original publication [1], all of the dynamic data sets were processed for each of the innovative condensing flow run cases in Table 2.1. The dynamic data average frequencies and amplitude values for select flow variables were averaged independently for each case. These averages, along with the average of the short-term time-averages (over $3-5$ seconds each) of the dynamic data for select flow variables for each case, are reported in Table 2.2. Out of these dynamic data sets, the ones processed for cases $\mathrm{C} 1$ - C4, C5 - C6, and $\mathrm{C} 10$ were selected for further analysis. For the boiling flow cases, all 10 dynamic data sets were processed for each case, and the resulting frequencies and amplitudes of the flow variables were averaged independently to obtain the entries in Table 2.3. One representative dynamic data set each from cases B1 and B2 was selected for further analysis. A brief description of the methods used to process the dynamic data sets and obtain amplitudes and frequencies of relevant flow variables follows.

A Fast Fourier Transform (FFT) analysis was performed on selected flow variables, and the amplitudes reported in tables $2.1-2.3$ are from the peaks in the magnitudes of the FFT results at the primary imposed pulsation frequency on the vapor, $\mathrm{f}_{\mathrm{P}: \mathrm{V}}$, entering the 
test-section, except for the N-IF boiling flow cases B1, B4, B6, and B7, for which the inadvertently imposed liquid pulsation frequency, $\mathrm{f}_{\mathrm{P}: \mathrm{L}}$, was used instead. For all the other boiling flow cases in Table 2.3, the values of $f_{P: L}$ were so close to the values of $f_{P: V}$ that they are represented by the same frequency in the Discrete Fourier Transform computed by means of FFT. Representative amplitudes for the imposed fluctuation (IF) cases in Tables $2.2-2.3$ are characterized as "low amplitude" (L-IF) or "high amplitude" (H-IF) depending on the low or high values of the amplitude associated with imposed fluctuations' pressure-difference $\left(\mathrm{a}_{\Delta \mathrm{p} \mid 0-40}\right)$ - which directly relates to flow rate fluctuation amplitudes and indirectly relates to absolute pressure $\mathrm{p}_{10}$ fluctuation amplitudes - relative to their representative mean value, $\overline{\Delta p}_{0-40}$. A third classification of the pressure-difference amplitude, "transition amplitude" (T-IF), is applied to cases for which the dynamic variation of heat-flux in time alternately displays features of L-IF and H-IF cases (see Fig. 2.18 and Fig. 3.13).

The uncertainties reported in the column headers of Tables $2.1-2.3$ for time-averaged values of the flow variables only apply to those mean values; instantaneous values of data taken at the low DAQ rate may vary outside of that range. Uncertainties are reported as absolute (a number without a \% symbol), relative (a number followed by a \% symbol), or mixed (both a plain numerical value and a percentage). Absolute uncertainties have the same units as the variable to which they are applied, and relative uncertainty values are applied as a percentage of the reported data values. To determine the uncertainty associated with any number with a mixed uncertainty, multiply the percent uncertainty by the reported data value of interest and divide by 100; then add to this quotient the absolute uncertainty, and that data value of interest is reported to \pm this sum. The confidence interval on all reported uncertainty values in Tables $2.1-2.3$ is $80-90 \%$, and it includes effects of working temperature on the sensors, where applicable, and signal leakage and noise in the FFT processing for the reported amplitude values. Calculations such as determination of saturation temperature, pressure at $x=40 \mathrm{~cm}$, and pressure-difference between the locations at $x=0 \mathrm{~cm}$ and $x=90 \mathrm{~cm}$ were performed for each case using instantaneous values of the relevant flow variables. 


\subsection{Results and discussions}

The experimental measurements allow determination of the hydro-dynamic (pressures, pressure-differences, flow rates, etc.) and thermal (inlet temperature, inlet saturation temperature, heat-exchange surface's temperature variations, etc.) boundary conditions for the condenser and boiler test-sections associated with operations depicted in Figs. $2.1 \mathrm{~b}$ and 2.2b. Since the heat-exchange surface's thermal conditions are known by the experimentally measured values of steady surface-temperature variations (this is done for both non-pulsatile N-IF and pulsatile T-IF and H-IF cases), the associated heat-flux variations on the heat-exchange surface can, in principal, either be theoretically/computationally calculated or experimentally measured. Because of the high cost associated with high-fidelity dynamic heat-flux measurements, heat-flux values were experimentally measured (by HFM-40 in Fig. 2.3) only for the $x=40 \mathrm{~cm}$ location. However, for the non-pulsatile N-IF condensing flow cases, a high-fidelity and rigorous computational solution technique was used ([22], [24]) to predict the heat-flux variations over the condensing surface. For several such cases, these predictions ([22], [24]) were found to be consistent (within $\pm 20 \%$ ) with approximately steady experimentally measured HFM-40 values.

\subsubsection{Results specific to condensing flows realized in the innovative arrangement of Fig. 2.1b}

The runs are numbered in the chronological order in which the data was taken, with data for run $\mathrm{C} 1$ being taken first and data for run $\mathrm{C} 10$ being taken last. These runs are classified by type with respect to imposed vapor pressure fluctuations, according to whether they are for no-imposed fluctuations (N-IF), low amplitude imposed fluctuations (L-IF), transition amplitude imposed fluctuations (T-IF), or high amplitude imposed fluctuations (H-IF).

All the data in Table 2.1, except for the vapor phase pulsation frequency $f_{P: V}$, were taken from averages in time of their values from the low DAQ rate data. Values of $f_{P: V}$ were taken by averaging the dominant frequency values (each corresponding to the highest 
local maximum FFT magnitude of variables $p_{10}$ and $\Delta p_{0-40}$ for a dynamic data set) from the FFTs for the 10 dynamic data sets acquired for each run (except for case C8, which used 11 dynamic data sets for the calculation of $\mathrm{f}_{\mathrm{P}: \mathrm{V}}$ and for the rest of the dynamic data reported in Table 2.2).

The saturation temperature of the incoming vapor for all of the reported cases in Table 2.1 is $58.01 \pm 0.15^{\circ} \mathrm{C}$. The pressure measured by APT-10 (see Fig. 2.3c) is designated as $p_{10}$, and it is used to represent the inlet pressure of the vapor supplied to the test-section. The total mass flow rate of vapor supplied to the condenser is designated as $\dot{\mathrm{m}}_{\mathrm{l}}=\dot{\mathrm{m}}_{\mathrm{l: \textrm {V }}}\left(\dot{\mathrm{m}}_{\mathrm{l}: \mathrm{L}}\right.$ in Fig. 2.4 was zero or negligibly small for all the cases in Table 2.1), and the total mass flow rate of liquid pumped out of the exit of the condenser is designated as $\dot{m}_{\mathrm{E}: \mathrm{L}}$ (see Fig. 2.4). Because quasi-steady or steady-in-the-mean (over sufficiently long time periods) conditions exist for reported data, the following relation must hold due to conservation of mass: $\dot{m}_{\mathrm{I}: \mathrm{V}}=\dot{m}_{\mathrm{R}: \mathrm{V}}+\dot{m}_{\mathrm{E}: \mathrm{L}}$, where $\dot{m}_{\mathrm{R}: \mathrm{V}}=\dot{m}_{\mathrm{E}: \mathrm{V}}$ (see Fig. 2.4) is the mass flow rate of vapor re-circulated. This means that the reported quasi-steady $\dot{m}_{E: L}$ represents the time-averaged rate of mass condensed within the test section.

The mean inlet and exit vapor velocity values (based on channel cross-sectional flow area) are denoted as $\mathrm{v}_{\mathrm{I}: \mathrm{V}}$ and $\mathrm{v}_{\mathrm{E}: \mathrm{V}}$, respectively. The value of $\mathrm{v}_{\mathrm{I}: \mathrm{V}}$ ranged from $1.13-1.15 \mathrm{~m} / \mathrm{s}$, and the value of $\mathrm{v}_{\mathrm{E}: \mathrm{V}}$ ranged from $0.63-0.80 \mathrm{~m} / \mathrm{s}$. The vapor velocity values which were reported in Table 1 of [1] were incorrect because the wrong channel gap height ( $2 \mathrm{~mm}$ instead of the correct $6 \mathrm{~mm}$, see Fig. 2.3.a) was used to compute the flow area of the vapor. The exit liquid velocity is negligibly small, so it is not reported here.

The inlet vapor temperature is reported as $\mathrm{T}_{\mathrm{I:V}}$, and it is measured by an exposed-tip thermocouple inserted into the vapor flow stream immediately upstream of the test-section inlet. The temperature at the $\mathrm{x}=0 \mathrm{~cm}$ location is reported as $\mathrm{T}_{0}$, and it ranges from $0-4^{\circ} \mathrm{C}$ below the saturation temperature. The exit liquid temperature is measured by a thermocouple probe of $1 / 16$ inch diameter which is inserted into the liquid flow 
stream approximately $6 \mathrm{~mm}$ underneath the heat-exchange surface in the exit liquid port, and its measured temperature is designated as $T_{E: L^{\circ}}$. The difference between the saturation temperature of the incoming vapor taken at time-averaged pressure $p_{10}$ and the spatially-averaged condensing-surface temperature is denoted as $\Delta T$. This is further divided into two parts: the temperature difference corresponding to only the first $40 \mathrm{~cm}$ of the test-section is denoted as $\Delta \mathrm{T}_{0-40}$, and the temperature difference corresponding to nearly the entire heat-exchange surface is denoted as $\Delta \mathrm{T}_{0-88.5}$. The thermocouple measuring condensing surface temperature closest to the exit of the test-section is located at $x=88.5 \mathrm{~cm}$, so this limits computation of the condensing surface temperature to $0 \leq x \leq 88.5 \mathrm{~cm}$.

The value (both time-varying and time-averaged) of heat-flux measured by a flush heat-flux meter (see HFM-40 in Fig. 2.3c) is denoted as q" ${ }_{40}$. Surface temperature at the HFM-40 $(x=40 \mathrm{~cm})$ location is measured by a thermocouple internal to the heat-flux meter probe, and its time-averaged value is designated by $T_{40}$. The pressure-difference value (both time-varying and time-averaged) measured by DPT-1 in Fig. 2.3a is designated as $\Delta \mathrm{p}_{0-40}$. The pressure difference over the first $90 \mathrm{~cm}$ of the test-section is calculated as $\Delta \mathrm{p}_{0-90}=\Delta \mathrm{p}_{0-40}+\Delta \mathrm{p}_{40-90}$, where $\Delta \mathrm{p}_{40-90}$ is the pressure-difference measured by DPT-2 in Fig. 2.3a. The absolute pressure at $x=40 \mathrm{~cm}$ can be estimated as $p_{40} \approx p_{10}-\Delta p_{0-40}$ or $p_{40} \approx p_{0}-\Delta p_{0-40}$. Its time-averaged value is not relevant because the uncertainty associated with time-averaged values of $p_{10}$ or $p_{0}$ is much larger than the time-averaged value of $\Delta p_{0-40}$. Nevertheless, amplitudes of this estimated $p_{40}$ value are more accurate and relevant; they are discussed later.

As one goes from N-IF to H-IF cases, Table 2.1 clearly shows that negative values of $\Delta p_{0-40}$ and $\Delta p_{0-90}$ change due to significant effects of pulsations on the vapor pressure-field within the condenser. The condenser pressure-difference measured by flush absolute pressure transducers APT-10 and APT-90 (see Fig. 2.3c) is also reported in Table 2.1 as $\Delta \mathrm{p}_{10-90}$ for purposes of comparison with values for boiling flows reported in Table 2.3. Values of $\Delta p_{10-90}$ tend to be larger than the values of $\Delta p_{0-90}$, perhaps because 
of pressure rises over the first $10 \mathrm{~cm}$ of the test-section due to rapid deceleration of the vapor near the onset of condensation, where the liquid film is thinnest and condensation rates are expected to be the highest. However, the differences may instead be due primarily to the higher uncertainties in the values of $p_{10}$ and $p_{90}$. 
Table 2.1.

Long-term (25 minutes) averages of selected flow variables from innovative flow condenser runs. The data were Shaded cells contain non-imposed pulsation frequency values.

\begin{tabular}{|c|c|c|c|c|c|c|c|c|c|c|c|c|c|}
\hline & $\mathbf{f}_{\mathrm{P}: \mathrm{V}}$ & $p_{10}$ & $\dot{\mathrm{m}}_{\mathrm{I}: \mathrm{V}}$ & $\dot{m}_{\mathrm{E}: \mathrm{L}}$ & $\mathrm{T}_{\mathrm{I}: \mathrm{V}}$ & $T_{0}$ & $T_{40}$ & $\mathrm{~T}_{\mathrm{E}: \mathrm{L}}$ & $\Delta T_{0-40}$ & $\Delta T_{0-88.5}$ & $q "{ }_{40}$ & $\Delta$ \\
\hline & & \pm 0.1 & \pm 0.5 & \pm 0.008 & \pm 0.001 & \pm 1 & \pm 1 & \pm 2 & \pm 1 & \pm 0.5 & \pm 0.4 & \pm 0.2 & \\
\hline Run & Type & {$[\mathrm{Hz}]$} & [kPa] & [g/s] & [g/s] & {$\left[{ }^{\circ} \mathrm{C}\right]$} & {$\left[{ }^{\circ} \mathrm{C}\right]$} & {$\left[{ }^{\circ} \mathrm{C}\right]$} & {$\left[{ }^{\circ} \mathrm{C}\right]$} & {$\left[{ }^{\circ} \mathrm{C}\right]$} & {$\left[{ }^{\circ} \mathrm{C}\right]$} & {$\left[\mathrm{W} / \mathrm{cm}^{2}\right]$} & \\
\hline C1 & $\mathrm{N}-\mathrm{IF}$ & 16.2 & 104.99 & 2.290 & 0.726 & 64 & 58 & 40 & 50 & 15.3 & 17.8 & 0.34 & \\
\hline C4 & T-IF & 10.6 & 105.01 & 2.295 & 1.012 & 59 & 54 & 41 & 54 & 15.8 & 17.1 & 0.51 & \\
\hline C3 & T-IF & 10.6 & 105.00 & 2.295 & 0.972 & 59 & 54 & 42 & 53 & 15.8 & 17.0 & 0.66 & \\
\hline C2 & $\mathrm{H}-\mathrm{IF}$ & 10.6 & 105.00 & 2.300 & 1.024 & 59 & 54 & 42 & 54 & 15.6 & 16.6 & 0.89 & \\
\hline C5 & $\mathrm{N}-\mathrm{IF}$ & 4.7 & 104.96 & 2.304 & 0.674 & 60 & 55 & 39 & 48 & 16.6 & 19.0 & 0.33 & \\
\hline C6 & L-IF & 28.3 & 104.99 & 2.300 & 0.794 & 60 & 55 & 40 & 50 & 16.6 & 18.8 & 0.34 & \\
\hline C7 & T-IF & 28.4 & 104.99 & 2.296 & 0.769 & 60 & 55 & 41 & 49 & 16.4 & 18.6 & 0.64 & \\
\hline C8 & $\mathrm{H}-\mathrm{IF}$ & 28.4 & 105.02 & 2.293 & 0.903 & 60 & 55 & 42 & 54 & 16.2 & 18.3 & 1.49 & \\
\hline C9 & $\mathrm{H}-\mathrm{IF}$ & 28.4 & 105.00 & 2.297 & 0.951 & 60 & 55 & 43 & 54 & 16.0 & 18.0 & 2.16 & \\
\hline C10 & H-IF & 28.5 & 105.01 & 2.301 & 0.876 & 60 & 56 & 44 & 51 & 15.5 & 17.4 & 3.18 & \\
\hline
\end{tabular}

Table 2.2.

Dynamic data averages (over average time-periods of $\overline{\Delta t}_{B}$ ) and amplitudes at frequency $f_{P: V}$ of selected flow variab condenser runs. Shaded cells contain non-imposed pulsation frequency values and corresponding flow variable ar

\begin{tabular}{|c|c|c|c|c|c|c|c|c|c|c|c|c|c|}
\hline & $\overline{\Delta \bar{t}_{B}}$ & $f_{\mathrm{P}: \mathrm{V}}$ & $p_{10}$ & $a_{p \mid 10}$ & $T_{40}$ & $q^{\prime \prime} 40$ & $a_{q^{\prime \prime} \mid 40}$ & $\Delta p_{0-40}$ & $a_{\Delta p \mid 0-40}$ & $\Delta \mathrm{p}_{0-90}$ & $a_{\Delta p \mid 0-90}$ & $\Delta p$ \\
\hline & & \pm 0.0005 & \pm 0.1 & \pm 0.5 & $\pm 0.5 \%$ & \pm 2 & \pm 0.2 & $\pm 6 \%$ & \pm 28 & $\pm 0.5 \%$ & \pm 43 & $\pm 0.7 \%$ & \\
\hline Run & Type & [s] & {$[\mathrm{Hz}]$} & [kPa] & {$[\mathrm{Pa}]$} & {$\left[{ }^{\circ} \mathrm{C}\right]$} & {$\left[\mathrm{W} / \mathrm{cm}^{2}\right]$} & {$\left[\mathrm{W} / \mathrm{cm}^{2}\right]$} & [Pa] & {$[\mathrm{Pa}]$} & [Pa] & [Pa] & \\
\hline C1 & $\mathrm{N}$-IF & 4.94315 & 16.2 & 105.01 & 54 & 40 & 0.36 & 0.002 & -76 & 16 & -53 & 61 & \\
\hline C4 & T-IF & 4.06065 & 10.6 & 104.93 & 1020 & 42 & 0.53 & 0.093 & -13 & 544 & -40 & 764 & \\
\hline C3 & T-IF & 4.25395 & 10.6 & 104.93 & 1119 & 43 & 0.71 & 0.253 & -7 & 611 & -38 & 831 & \\
\hline $\mathrm{C} 2$ & $\mathrm{H}-\mathrm{IF}$ & 4.44910 & 10.6 & 104.98 & 1178 & 43 & 0.95 & 0.521 & -32 & 667 & -54 & 879 & \\
\hline C5 & $\mathrm{N}-\mathrm{IF}$ & 4.74875 & 4.7 & 105.02 & 49 & 40 & 0.34 & 0.002 & -53 & 2 & -26 & 11 & \\
\hline C6 & L-IF & \begin{tabular}{|l|}
4.15150 \\
\end{tabular} & 28.3 & 105.03 & 211 & 40 & 0.35 & 0.003 & -58 & 540 & -28 & 426 & \\
\hline C7 & T-IF & 3.68490 & 28.4 & 105.01 & 326 & 41 & 0.62 & 0.069 & -62 & 763 & -15 & 645 & \\
\hline C8 & $\mathrm{H}-\mathrm{IF}$ & 3.90882 & 28.4 & 105.21 & 474 & 43 & 1.51 & 0.650 & -69 & 1002 & -7 & 883 & \\
\hline C9 & H-IF & 4.33820 & 28.4 & 105.00 & 593 & 44 & 2.19 & 1.112 & -78 & 1171 & 0 & 1059 & \\
\hline C10 & H-IF & 3.82290 & 28.5 & 104.95 & 811 & 45 & 3.34 & 1.641 & -98 & 1442 & 12 & 1348 & \\
\hline
\end{tabular}



The values in Table 2.2 were taken from the dynamic data sets acquired at a rate of $2000 \mathrm{~Hz}$ for periods of 5 seconds each. Ten of these $5 \mathrm{~s}$ data sets were acquired for each run, except for case C8, for which 11 data sets were acquired. Subsets of this $5 \mathrm{~s}$ period were selected to minimize "leakage" in the computation of the FFT. The average values and amplitudes (from FFT magnitude data) of the relevant flow variables at frequency $f_{P: V}$ (computed from their FFTs) for the 10 (11 for case C8) data sets were then averaged. These averages are the values reported in Table 2.2. The long-term averages of the same flow variables, with the exception of the calculated film thickness values $\delta_{40}$, are reported in Table 2.1.

The only values that were computed differently were the time-averaged values of the film thickness, $\delta_{40}$. Because the heat-flux values $q^{\prime \prime}{ }_{40}(t)$ and surface temperature values $T_{40}$ contained significant amounts of electrical noise, some computed estimates of $\delta_{40}$ fell outside of the physically reasonable range of $0 \leq \delta_{40} \leq 2000 \mu \mathrm{m}$. With these values removed, the remaining values within each data block were averaged through trapezoidal rule integration with respect to time, dividing by the time period for that data set, $\Delta \mathrm{t}_{\mathrm{B}}$, to obtain an average film thickness. Ten (11 for case C8) of these averaged film thickness values, corresponding to each of the ten ( 11 for case C8) data sets, were then averaged to obtain the average value of $\delta_{40}$ for the run, reported in Table 2.2.

Flow visualization data were recorded in pictures and video. Representative videos are included in the accompanying data disc. Video 2-1 corresponds to N-IF case C5 in Tables $2.1-2.2$. Video 2-2 corresponds to H-IF case C10 in Tables $2.1-2.2$. Notice the effect of pulsations on the interface, adding rigorous time-variations in the interface location for $\mathrm{H}$-IF case $\mathrm{C} 10$ in contrast to $\mathrm{N}$-IF case $\mathrm{C} 5$, which shows the waves on the interface which are naturally present without the addition of imposed pulsations. Note that the time-variations in wave peak locations visible in Video 2-2 are actually faster than they appear in the video. The camera frame rate was 30 frames per second, and the pulsations were at $28.5 \mathrm{~Hz}$, so the effect of the pulsations on the liquid-vapor interface is "aliasing" into the video frames for Video 2-2. 


\subsubsection{Discussion of results for condensing flows in the innovative arrangement of Fig. 2.1b}

Figures 2.6 - 2.11 represent the variation of relevant test-section flow variables over long time-durations (minutes) for a sampling of the innovative condensing flow cases $\mathrm{C} 1-\mathrm{C} 10$ in Table 2.1. Each sample in Figs. 2.6 - 2.11 is the average of 25 samples of the original data, giving them an effective sampling frequency of around $0.036 \mathrm{~Hz}$, which eliminates the majority of random noise. This digital filtering method also significantly reduces fluctuations arising from aliasing of time-variations in the heat-fluxes and pressure-differences at the pulsation frequency, which was verified by using values of $f_{P}$ from the dynamic data in conjunction with the averaged sampling frequency of the low DAQ rate data to estimate the frequency into which the imposed pressure fluctuations would alias for the cases in Tables $2.1-2.2$.

For representative N-IF, L-IF, and H-IF cases marked as C1, C4, and C2 in Table 2.1, Figs. $2.6-2.8$ show the long-time (with local time-averaging) behavior of several variables as obtained through the low DAQ rate time-records for these variables. Fig. 2.6 shows that the mean inlet mass flow rates $\dot{m}_{\mathrm{l:V}}$ and absolute values of $\Delta \mathrm{p}_{0-40}$ remain approximately constant as imposed fluctuation amplitudes increase, but from Table 2.1 it is clear that for significant levels of imposed pulsations (T-IF cases C4 - C3, and $\mathrm{H}-\mathrm{IF}$ case $\mathrm{C} 2$ ) the absolute value of $\Delta \mathrm{p}_{0-40}$ significantly reduces from the N-IF case $\mathrm{C} 1$. This is because the shift in $\Delta \mathrm{p}_{0-40}$ is small compared with the fluctuations visible in Fig. 2.6 and the value of $a_{\Delta p \mid 0-40}$. Note that the imposition of pressure/flow pulsations in the vapor phase increases the exit liquid mass flow rate $\dot{m}_{\mathrm{E}: \mathrm{L}}$, which means that more condensation is occurring within the test-section for the T-IF and H-IF cases. 


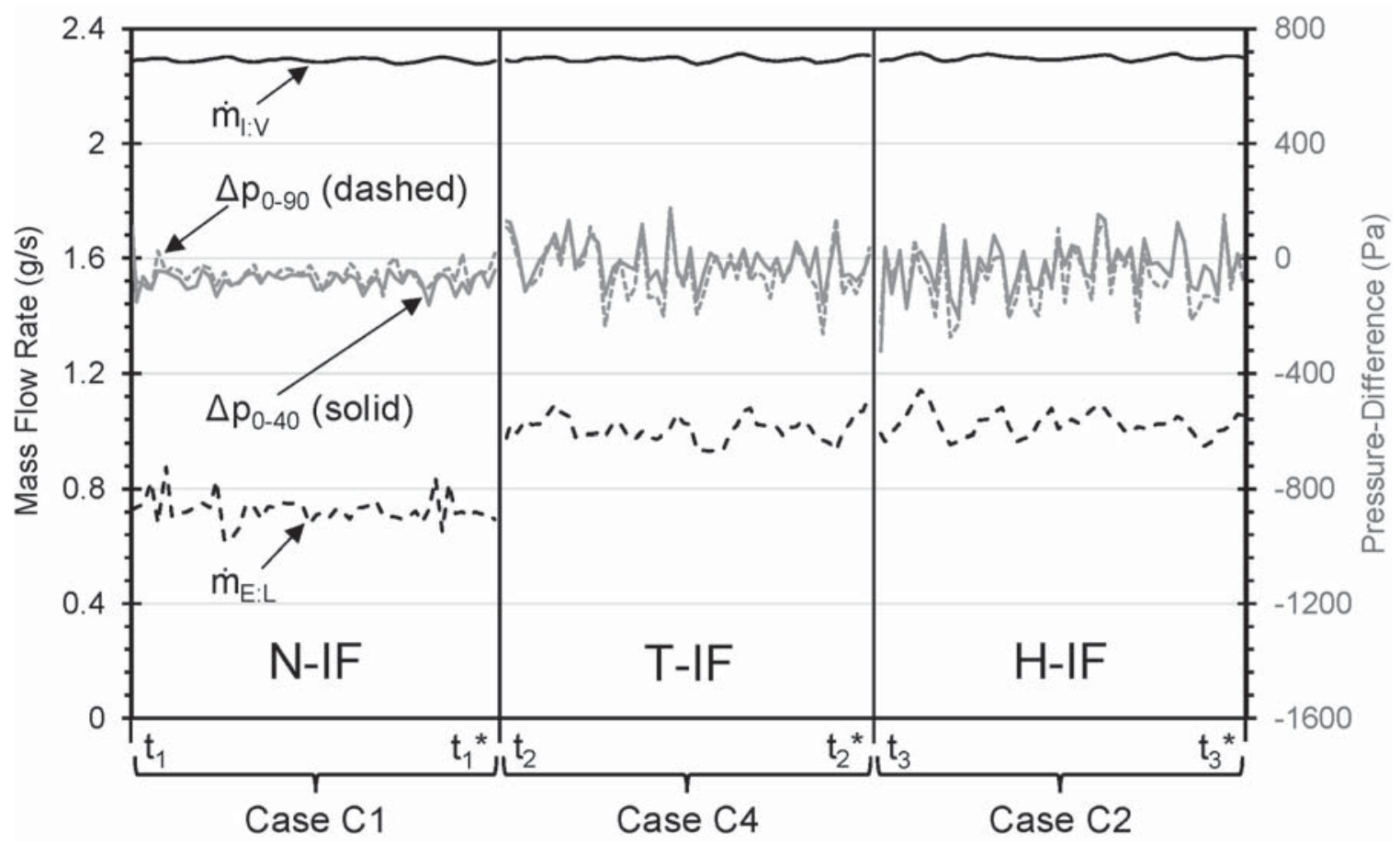

Figure 2.6. Mass flow rates and pressure-differences associated with runs $C 1, C 4$, and $C 2$ in Tables $2.1-2.2$. The imposed pulsation frequency was $10.6 \mathrm{~Hz}$. The data shown for each run were collected over time periods of 25 minutes. Each sample in the graph was obtained by averaging 25 contiguous samples from the low DAQ rate data.

Figure 2.7 shows long-term oscillations in the absolute pressures at the $\mathrm{X}=10 \mathrm{~cm}$ and $X=90 \mathrm{~cm}$ locations. These oscillations are probably the results of mild destabilization of the liquid pump ( $\mathrm{P}_{1}$ in Fig. 2.4) feedback control due to the choice of PID control parameters, influence of inadvertent liquid removal pulsations caused by the pump, and noise in the inlet pressure $p_{10}$ reading (which served as the process variable for the PID control of the pump). Note that for cases $\mathrm{C} 4$ and $\mathrm{C} 2$, these pressure oscillations are significantly worse; this is caused by aliasing of the $p_{10}$ variations at the pulsation frequency $f_{P: V}$ into the $0.9 \mathrm{~Hz}$ data acquisition rate for transducer APT-10 (Fig. 2.3c), and its influence on the PID control of pump $\mathrm{P}_{1}$.

Also from Fig. 2.7, it is clear that the mean values of $x=40 \mathrm{~cm}$ surface-temperature $T_{40}$ increase with increasing amplitudes of imposed fluctuations at $\mathrm{f}_{\mathrm{P}: \mathrm{V}}=10.6 \mathrm{~Hz}$ in Table 2.1. The reduction in the $\mathrm{x}=0 \mathrm{~cm}$ surface temperature $\mathrm{T}_{0}$ for cases $\mathrm{C} 4$ and $\mathrm{C} 2$ 
relative to $\mathrm{N}-\mathrm{IF}$ case $\mathrm{C} 1$ indicates that the onset of condensation has moved slightly upstream of the designated 'inlet' location at $\mathrm{x}=0 \mathrm{~cm}$, resulting in a positive fluid-to-surface temperature difference at that location (note that $\mathrm{T}_{\text {Sat }} \approx 58{ }^{\circ} \mathrm{C}$ for cases $\mathrm{C} 1-\mathrm{C} 10)$. The increase in the $\mathrm{T}_{88.5}$ surface temperature with increased level of imposed pulsations indicates that the heat-flux near the exit of the test-section is increasing through the fixed thermal resistances of the condensing plate (Fig. 2.3c), thermoelectric modules (Figs. 2.3c-2.4), and heat sinks (Fig. 2.4) around that location.

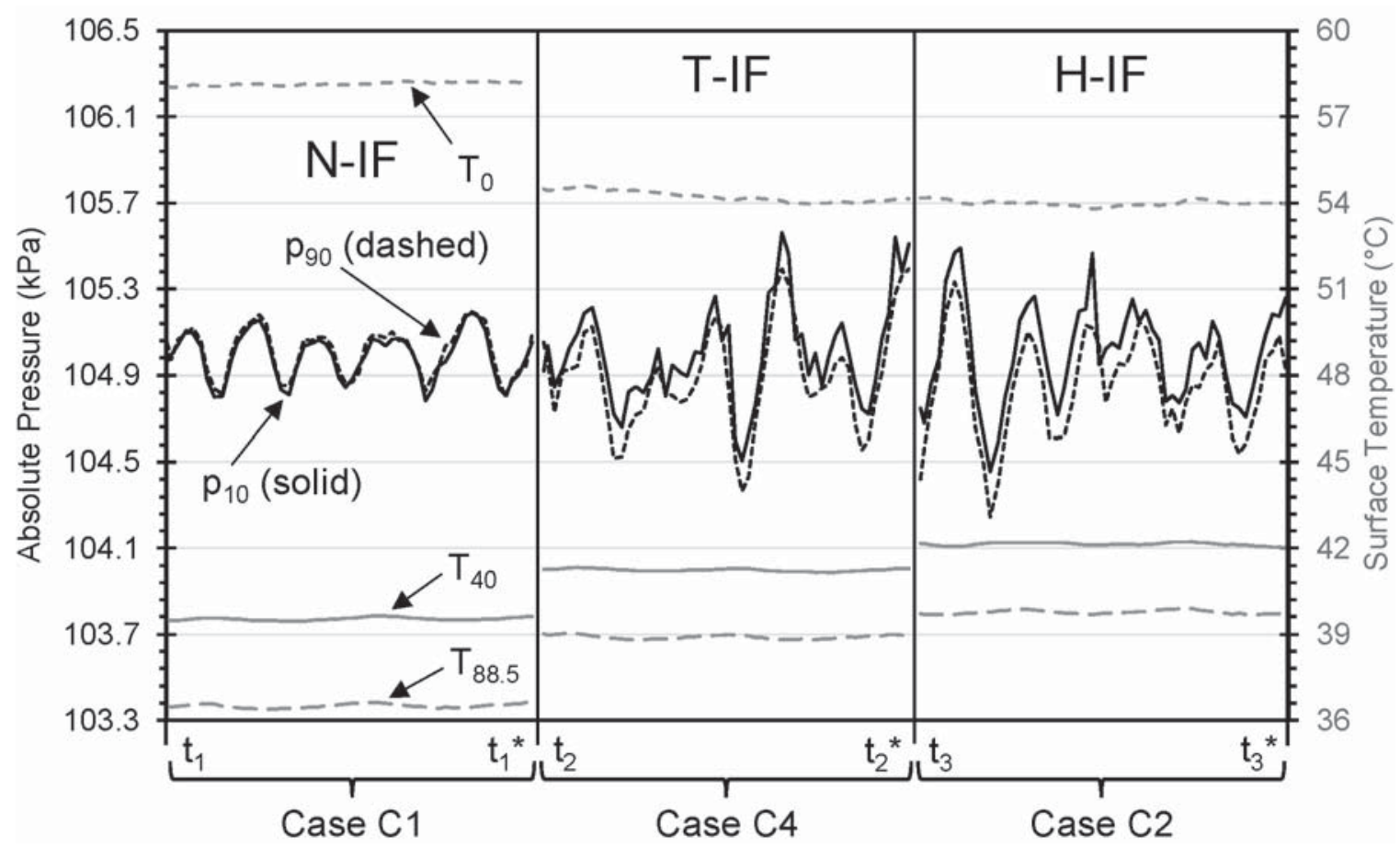

Figure 2.7. Absolute pressures and representative surface temperatures associated with runs C1, C4, and C2 in Tables 2.1 - 2.2. The imposed pulsation frequency was $10.6 \mathrm{~Hz}$. The data shown for each run were collected over time periods of 25 minutes. Each sample in the graph was obtained by averaging 25 contiguous samples from the low DAQ rate data.

Figure 2.8 shows that the heat-flux $\mathrm{q}_{40}$ increases greatly (by approximately $165 \%$ ) and is accompanied by an approximately $14 \%$ decrease in the local fluid-to-surface temperature difference $\Delta T_{40}$. At the $x=40 \mathrm{~cm}$ location, the increase in the surface temperature $T_{40}$ of Fig. 2.7 (discussed previously) and corresponding reduction in $\Delta T_{40}$ of Fig. 2.8, for the H-IF case C2, is a result of the increased heat-flux and the passive 
cooling condition used. The higher surface temperature is required to drive the increased heat-flux through the conductive thermal resistance of the heat-flux meter and stainless steel plate. Note the more moderate reduction in $0-88.5 \mathrm{~cm}$ temperature-difference $\Delta \mathrm{T}_{0-88.5}$ in Fig. 2.8 due to a lesser (but still very significant) heat-flux enhancement over the length of the test-section. However, the $0-40 \mathrm{~cm}$ temperature-difference $\Delta T_{0-40}$ does not change much, suggesting negligible heat-flux enhancement over the region of the test-section upstream of $x=40 \mathrm{~cm}$. Thus the increase in time-averaged heat flux for case $\mathrm{C} 2$ seems to be the greatest for $\mathrm{x} \geq 40 \mathrm{~cm}$. The estimated total (neglecting acoustic energy associated with the vapor pulsations) heat-flux $\mathrm{q}_{\text {total }}^{\prime *}$ depends primarily on the rate of condensation, measured by $\dot{m}_{\mathrm{E}: \mathrm{L}}$ through the relation $\mathrm{q}_{\text {total }}{ }^{*} \approx \mathrm{q}_{\text {condensed }}^{\prime \prime}=\dot{m}_{\mathrm{E}: \mathrm{L}} \cdot \mathrm{h}_{\mathrm{fg}}$, where $h_{f g}$ is the enthalpy of vaporization of FC-72. It has a minor increase with the imposition of vapor pressure/flow pulsations. Reasons for this are discussed later.

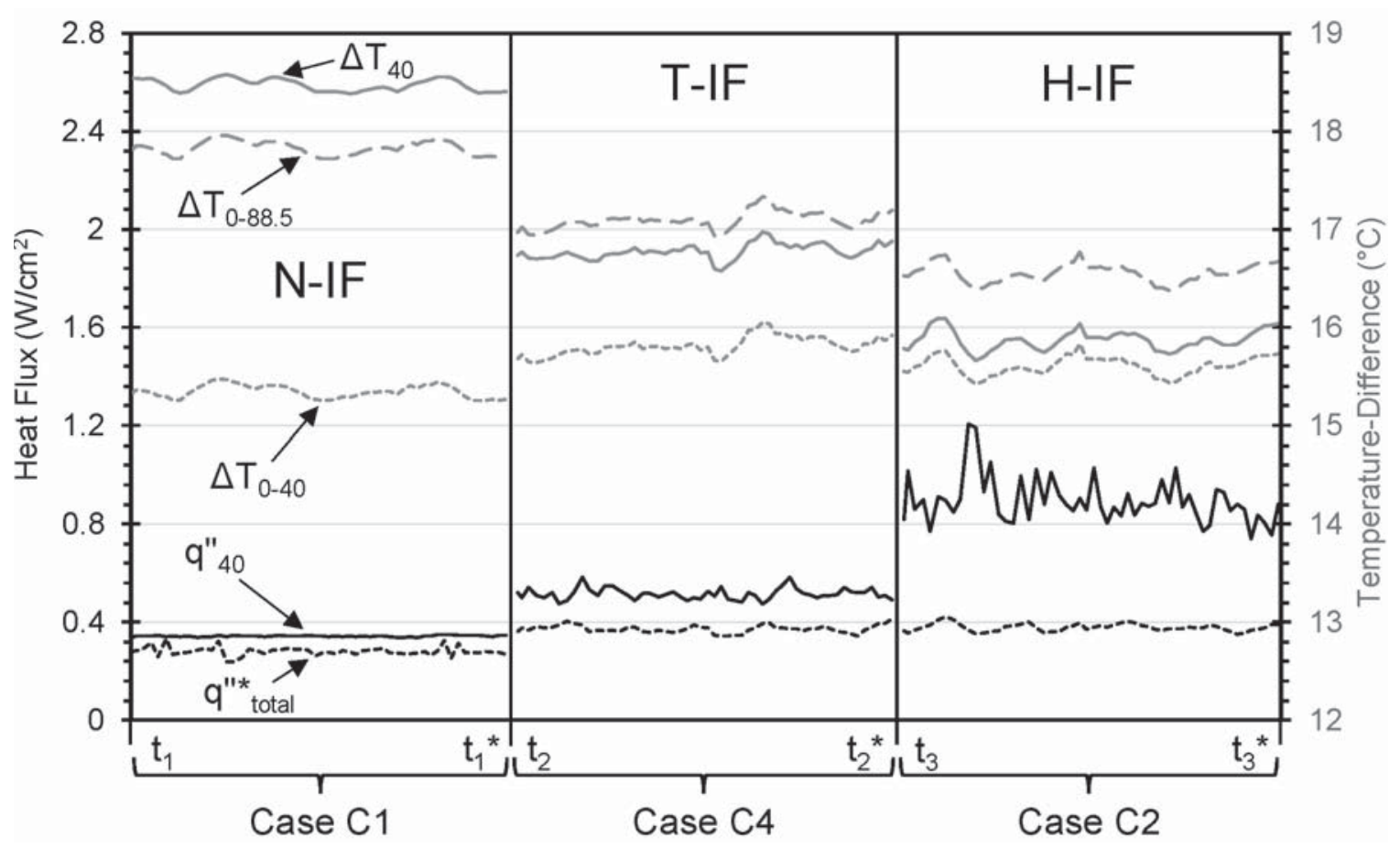

Figure 2.8. Heat-fluxes at $x=40 \mathrm{~cm}$ and representative temperature-differences associated with runs $\mathrm{C} 1, \mathrm{C} 4$, and $\mathrm{C} 2$ in Tables $2.1-2.2$. The imposed pulsation frequency was $10.6 \mathrm{~Hz}$. The data shown for each run were collected over time periods of 25 minutes. Each sample in the graph was obtained by averaging 25 contiguous samples from the low DAQ rate data. 
For representative N-IF, L-IF, and H-IF cases marked as C5, C6, and C10 in Table 2.1, Figs. $2.9-2.11$ show the long-time (with local time-averaging) behavior of several variables as obtained through the low DAQ rate time-records for these variables. Fig. 2.9 shows that the mean inlet mass flow rates $\dot{m}_{\mathrm{l}: \mathrm{V}}$ and absolute values of $\Delta \mathrm{p}_{0-40}$ remain approximately constant in the mean as imposed fluctuation amplitudes increase, but from Table 2.1 it is clear that as $a_{p \mid 10}$ increases, the absolute value of $\Delta p_{0-40}$ also increases from the N-IF Run C5 to the first H-IF case, Run C8. This is because the shift in $\Delta p_{0-40}$ is small compared with the fluctuations visible in Fig. 2.9 and the value of $a_{\Delta p \mid 0-40^{\circ}}$.

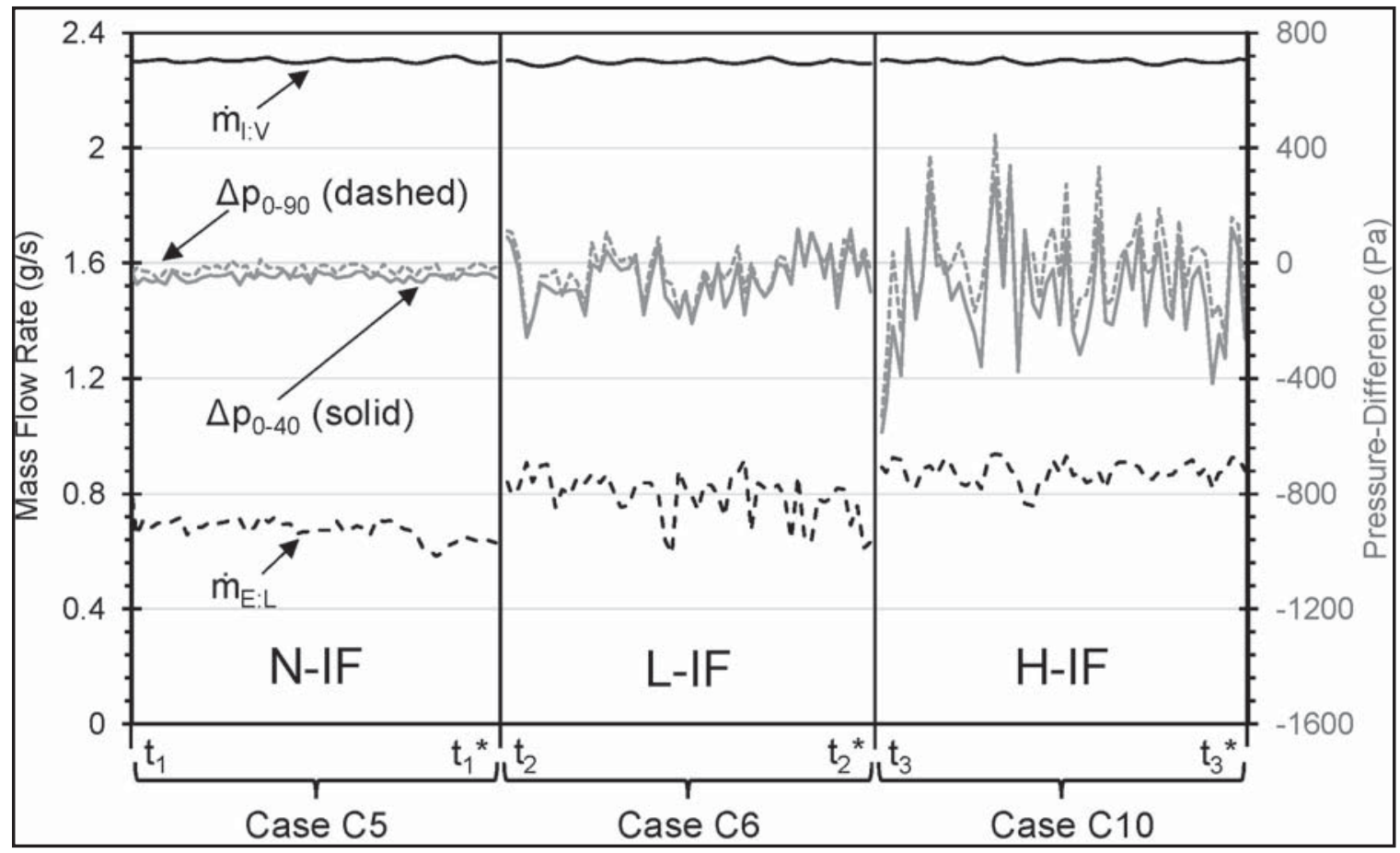

Figure 2.9. Mass flow rates and pressure-differences associated with runs $\mathrm{C} 5, \mathrm{C} 6$, and $\mathrm{C} 10$ in Tables $2.1-2.2$. The imposed pulsation frequency was around $28.4 \mathrm{~Hz}$. The data shown for each run were collected over time periods of 25 minutes. Each sample in the graph was obtained by averaging 25 contiguous samples from the low DAQ rate data.

Figure 2.10 shows long-term oscillations in the absolute pressures at the $x=10 \mathrm{~cm}$ and $x=90 \mathrm{~cm}$ locations. These oscillations are probably the results of factors previously discussed for Fig. 2.7. In contrast to cases $\mathrm{C} 4$ and $\mathrm{C} 2$, the pressure oscillations for imposed fluctuation cases C6 and C10 remain similar in magnitude to those for N-IF case 
C5; this is probably because the $p_{10}$ variations at the different pulsation frequency $f_{P: V}$ "aliased" into a different frequency in the $0.9 \mathrm{~Hz}$ data acquisition rate for transducer APT-10 (Fig. 2.3c) - one which could be handled better by the PID control for pump $P_{1}$. Note the especially large excursion in $p_{10}$ and $p_{90}$ in Fig. 2.10 near time $t=t_{1}{ }^{*}$; this may be due to a system transient.

Figure 2.10 also shows that the mean values of temperature $\mathrm{T}_{40}$, increase with increasing amplitudes of imposed fluctuations at $\mathrm{f}_{\mathrm{P}: \mathrm{V}} \approx 28.4 \mathrm{~Hz}$ in Table 2.1. To a lesser extent, the mean values of $T_{88.5}$ also increase with the imposition of pressure / flow rate pulsations, indicating a mild enhancement in heat-flux transferred to the condensing plate near the exit of the test-section for reasons discussed previously.

Note the abrupt drop in $\mathrm{T}_{88.5}$ near time $\mathrm{t}_{1}{ }^{*}$ in Fig. 2.10; this may be due to a destabilization of the annular flow regime (and possible transition to plug/slug regime) near the exit of the test-section for these times, or it may be due to a change in thermal resistance of the thermoelectric modules centered at $x=85 \mathrm{~cm}$ and $x=95 \mathrm{~cm}$ (see TEC-9 and TEC-10 in Fig. 2.3c). Of these explanations, the first is more likely, as manual data and notes taken during the experiments do not indicate a change in operation of any thermoelectric modules at those times; all the TECs remained turned off in the open-circuit electrical configuration.

The values of inlet surface temperature $T_{0}$ remain slightly below the saturation temperature, $\mathrm{T}_{\text {Sat }} \approx 58^{\circ} \mathrm{C}$, indicating that the onset of condensation is slightly upstream of the $\mathrm{x}=0 \mathrm{~cm}$ location for all three cases. 


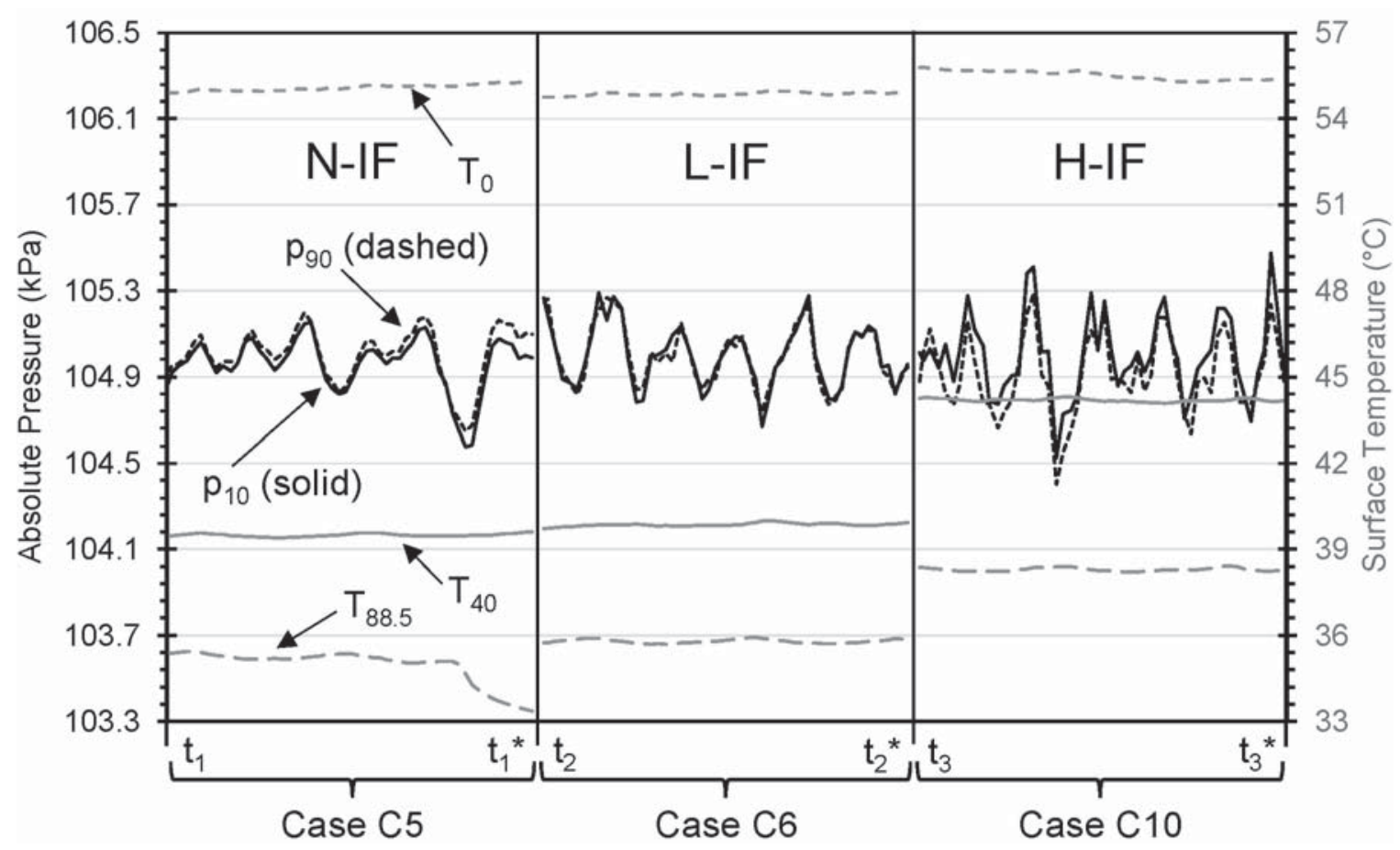

Figure 2.10. Absolute pressures and representative surface temperatures associated with runs C5, C6, and C10 in Tables $2.1-2.2$. The imposed pulsation frequency was around 28.4 Hz. The data shown for each run were collected over time periods of 25 minutes. Each sample in the graph was obtained by averaging 25 contiguous samples from the low DAQ rate data.

Figure 2.11 shows that the heat-flux q" ${ }_{40}$ increases by approximately $900 \%$ for case $\mathrm{C} 10$ relative to case $\mathrm{C} 5$ (much more than $\mathrm{q}_{40}{ }_{40}$ increases for case $\mathrm{C} 2$ relative to case $\mathrm{C} 1$ in Fig. 2.8) and is accompanied by an approximately $25 \%$ decrease in the local fluid-to-surface temperature-difference $\Delta \mathrm{T}_{40}$. At the $\mathrm{x}=40 \mathrm{~cm}$ location, the increase in the surface-temperature $\mathrm{T}_{40}$ of Fig. 2.10 and the corresponding reduction in $\Delta \mathrm{T}_{40}$ of Fig. 2.11, for the H-IF case C10, is a result of the increased heat-flux and the passive cooling condition used. The higher surface temperature is required to drive the increased heat-flux through the conductive thermal resistance of the heat-flux meter and stainless steel plate. As discussed previously for Case $\mathrm{C} 2$ in Fig. 2.8, temperature-difference $\Delta \mathrm{T}_{0-88.5}$ variations in Fig. 2.11 indicate mild enhancement in heat-flux transferred to the condensing plate over $0 \leq \mathrm{x} \leq 88.5 \mathrm{~cm}$ for H-IF case $\mathrm{C} 10$, and very small increases in $\mathrm{q}_{\text {total }}^{\prime *}$ are observed in Fig. 2.11 when the pressure / flow rate pulsations are imposed. However, in contrast to the behavior observed in Fig. 2.8 with $\mathrm{f}_{\mathrm{P}: \mathrm{V}}=10.6 \mathrm{~Hz}$, there is 
a noticeable reduction in the value of the $0-40 \mathrm{~cm}$ temperature-difference $\Delta \mathrm{T}_{0-40}$ caused by the imposition of the pressure / flow rate pulsations for case C10 in Fig. 2.11, indicating that mild enhancement of heat-flux into the condensing surface also occurs over $0 \leq \mathrm{x} \leq 40 \mathrm{~cm}$. Reasons for this are discussed later.

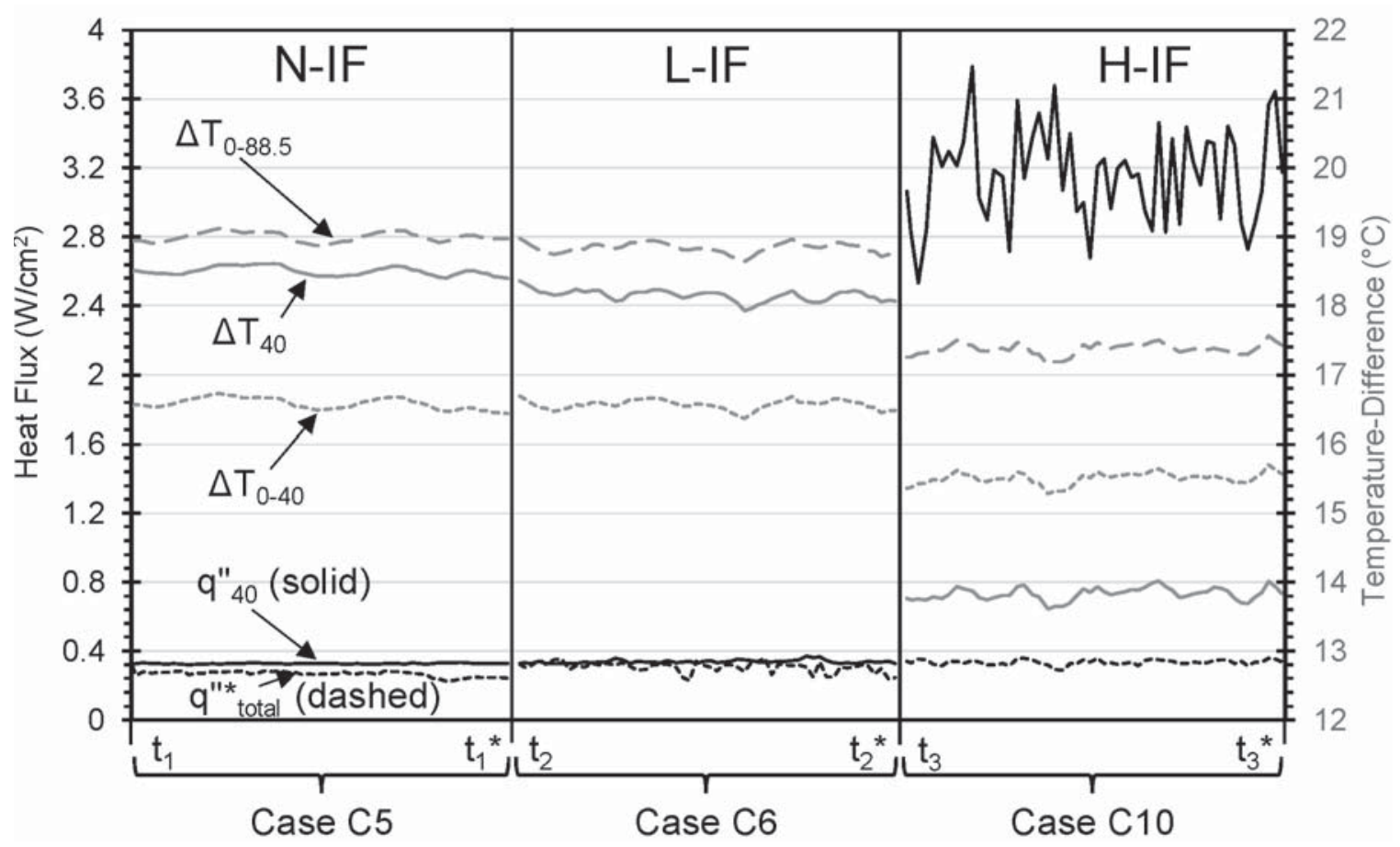

Figure 2.11. Heat-fluxes at $x=40 \mathrm{~cm}$ and representative temperature-differences associated with runs $\mathrm{C} 5, \mathrm{C} 6$, and $\mathrm{C} 10$ in Tables 2.1 - 2.2. The imposed pulsation frequency was around 28.4 Hz. The data shown for each run were collected over time periods of 25 minutes. Each sample in the graph was obtained by averaging 25 contiguous samples from the low DAQ rate data.

Figures $2.12-2.13$ show long-term averages in time of the surface temperature profiles within the test-section for cases $\mathrm{C} 1-\mathrm{C} 10$. As discussed previously, because of the cooling condition used and the thermal resistances (of the stainless steel plate of Fig. 2.3c, the thermoelectric modules (TECs) of Figs. 2.3c and 2.4, and the heat-sinks of Fig. 2.4), increases in surface temperature at a particular distance ' $x$ ' indicate increased heat-flux into the condensing plate at that location. 
However, as shown by the concurrent measurements of $\mathrm{q}^{\prime \prime}{ }_{40}$ and $\mathrm{T}_{40}$ from the heat flux meter at the $x=40 \mathrm{~cm}$ location in Figs. 2.8 and 2.11, the relationship between heat-flux and surface-temperature is not linear (percentage increase in heat-flux is greater numerically than percentage increase in surface-temperature). This is likely due to the complex path of heat flow through the condensing plate caused by the difference between the individual heat removal areas underneath the condensing plate and the continuous strip along which heat is entering the top surface of the condensing plate from the condensing FC-72 (see description in section 3.4.3.2) - requiring 3-dimensional conduction of heat within the condensing plate of Fig. $2.3 \mathrm{c}$.

The heat leakage to the ambient air by natural convection from the outside surfaces of the test-section may also influence the relationship between condensing surface temperature and condensing surface heat-flux if it is not a negligible percentage of the total heat load. This is because natural convection thermal resistances are not constant with respect to heat-flux, and significant natural convection effects would influence the paths of heat flow through the condensing plate of Fig. 2.3c. Nevertheless, it is expected that the natural convection heat losses would be significantly lower than the heat rejected to the water-cooled heat sinks of Fig. 2.4 because of the use of insulation around and under the condensing plate, along with the fact that natural convection coefficients are typically low for the range of surface-to-ambient temperature-differences (approximately $10-30{ }^{\circ} \mathrm{C}$ ) experienced by the exterior of the test-section. 


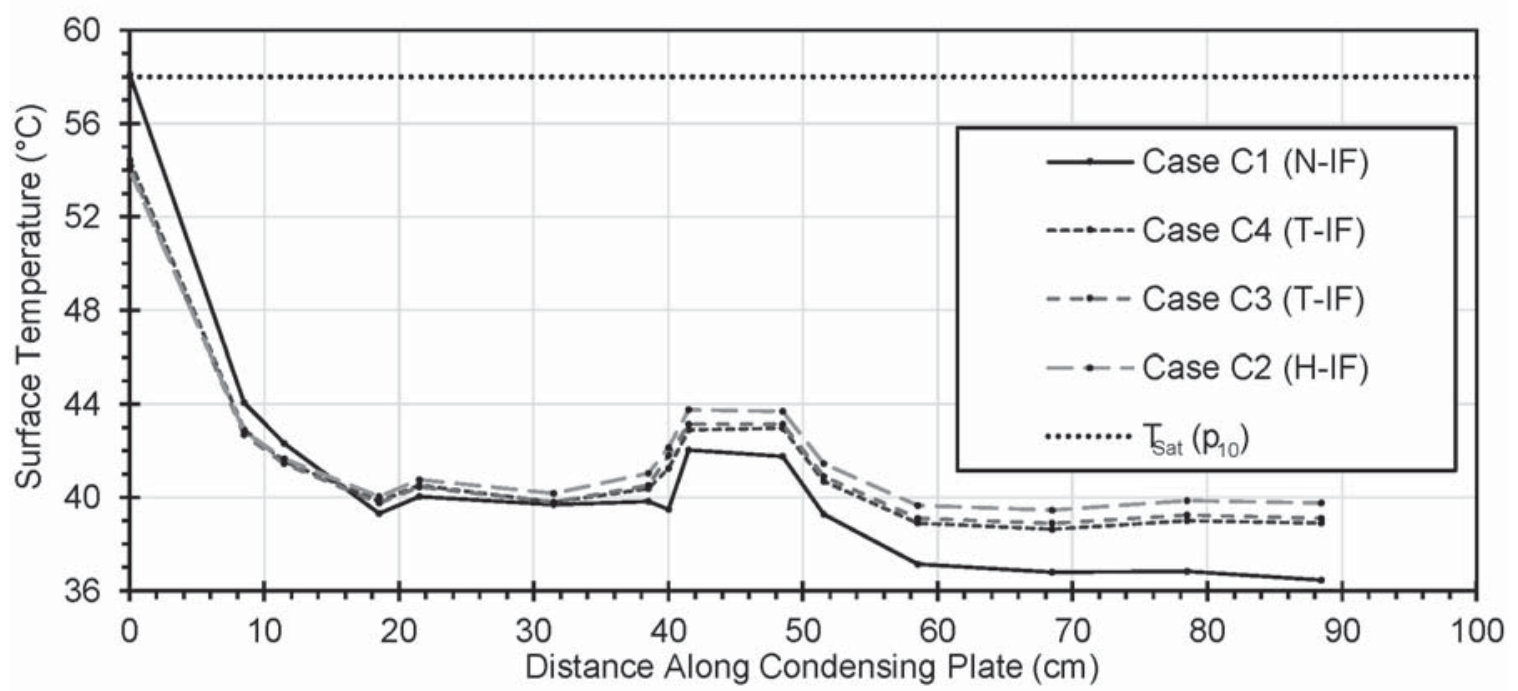

Figure 2.12. Variation of time-averaged surface temperature with distance from the test-section inlet for innovative condensation experiments for which the imposed pulsation frequency was 10.6 Hz. The average saturation temperature is shown for reference. Note the 'node-like' locations near $x=16 \mathrm{~cm}$ and $x=32 \mathrm{~cm}$ and the 'anti-node-like' locations near $x=20 \mathrm{~cm}$ and the near exit of the test-section.

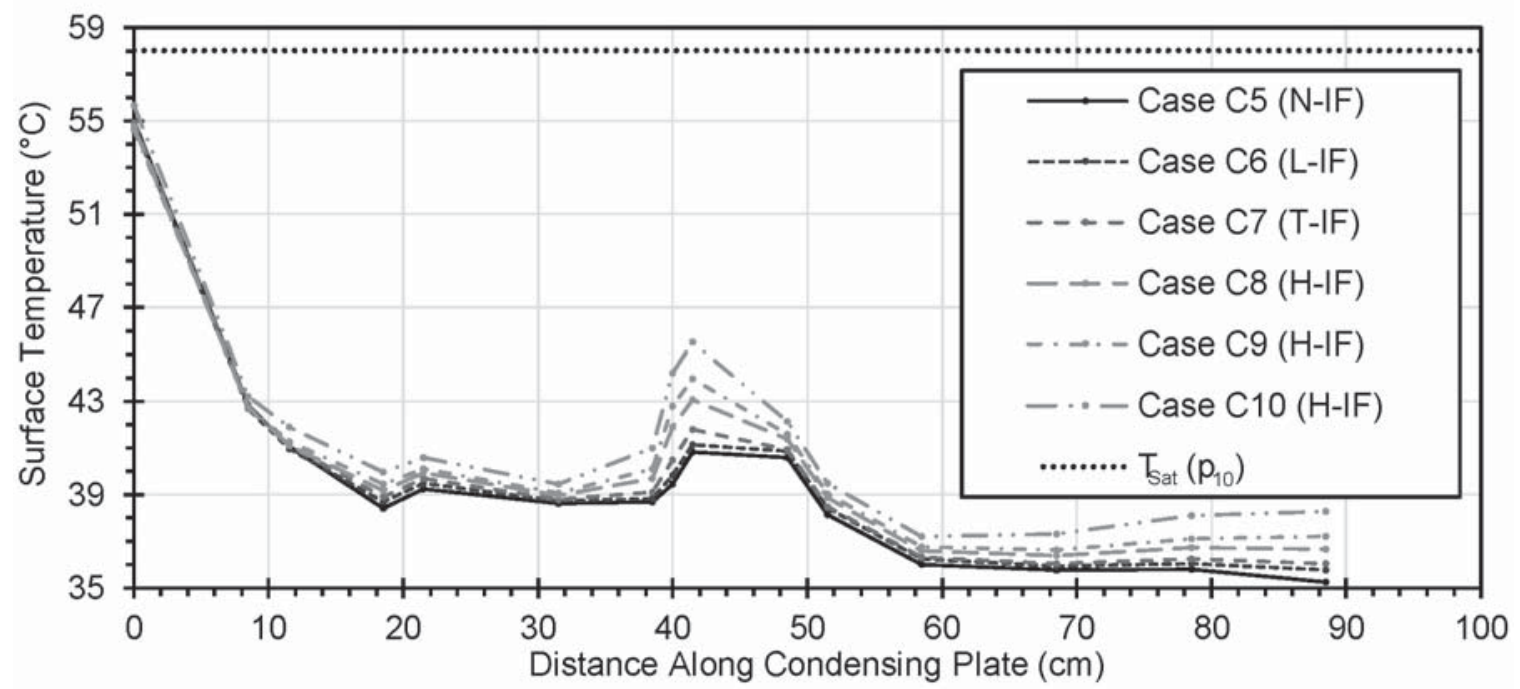

Figure 2.13. Variation of time-averaged surface temperature with distance from the test-section inlet for innovative condensing flow experiments for which the imposed pulsation frequency was around $28.4 \mathrm{~Hz}$. The average saturation temperature is shown for reference. Note the 'node-like' locations near $x=8 \mathrm{~cm}, x=32 \mathrm{~cm}$, and $x=58 \mathrm{~cm}$. 'Anti-node-like' locations are visible near $x=20 \mathrm{~cm}, x=42 \mathrm{~cm}$, and near the exit of the test-section. 
Similar to the surface temperature at the $40 \mathrm{~cm}$ location measured by the heat-flux meter (HFM-40 in Fig. 2.3c), the other surface temperatures in Figs. $2.12-2.13$ also tend to increase in other locations along the stainless steel plate, suggesting the presence of enhancement in heat-flux entering the plate from the condensing fluid over a significant portion of the condenser length. Although an energy balance analysis yields a more modest, but very significant $14-35 \%$ increase in total heat rejection $\left(q^{\prime \prime}\right.$ total in Figs. 2.8 and 2.11) from the fluid (not including acoustic energy dissipated from the fluctuations), note that the corresponding reduction in vapor-to-surface temperature-difference $(\Delta T)$ in Fig. 2.13 along most of the test-section length (and reduction in average $\Delta T_{0-88.5}$ of about $10 \%$ from case C5 to case C10 in Fig. 2.11) is also desirable for practical applications. Future and ongoing experiments for the properly controlled innovative configuration in Fig. 2.1b with improved flow controls (possibly with the inclusion of a flow-induced vibrating end-plate of the type suggested in Fig. 2.2b for innovative boiling flows) may ensure significant reduction in condensing side thermal resistance throughout the test-section.

The discrepancy between the very large enhancement in heat-flux measured by the heat flux meter at the $x=40 \mathrm{~cm}$ location and the much smaller overall heat-flux enhancement (estimated by a time-averaged thermodynamic energy balance of the temperatures and flow rates of the condensing fluid) may be attributed to the additional enthalpy of the incoming pulsatile vapor due to the mechanical energy associated with its pressure and flow pulsations, a portion of which must also be rejected to the condensing surface. Because no reliable method currently exists for obtaining concurrent time-varying values of pressure and mass or volume flow rate of the $\mathrm{FC}-72$ vapor near the $\mathrm{x}=0 \mathrm{~cm}$ location and near the test-section exit $(x>88.5 \mathrm{~cm})$, this mechanical energy associated with the pulsations is unknown, so it was not possible to incorporate it into the energy balance used to calculate $\mathrm{q}^{\prime *}{ }_{\text {total }}$. Because the exact nature of dependence of surface temperatures on heat-flux for locations other than at $x=40 \mathrm{~cm}$ is unknown, the true total heat-flux transferred from the fluid to the condensing plate (over the entire length of the condenser) $\mathrm{q}_{\text {total }}$, and the portion of this heat-flux coming from the mechanical energy in the 
pulsations, cannot be estimated for the experiments reported here.

Future experiments with improved instrumentation are expected to allow estimation of the percentage of enhancement in heat-flux which is due to the additional mechanical energy associated with the imposed pulsations, and to provide data which may suggest how to optimize the pulsatile condensing (or boiling) flow by reducing this percentage of the overall enhancement in heat-flux at the heat-exchange surface. The portion of enhanced heat-flux which is due to the mechanical energy associated with the imposed pulsations comes from energy which must be supplied by the pulsator, and thus it is generally not beneficial from a system design perspective.

For the N-IF and H-IF cases in Figs. 2.6 - 2.11, at 1 minute intervals from the start of the run, 10 higher DAQ rate $(2000 \mathrm{~Hz})$ dynamic data sets were acquired (except for case C8, for which 11 dynamic data sets were acquired). Time-domain averaged (between multiple dynamic data sets acquired for the same case) values of representative flow variables taken from this dynamic data are shown in Figs. $2.14-2.19$. 



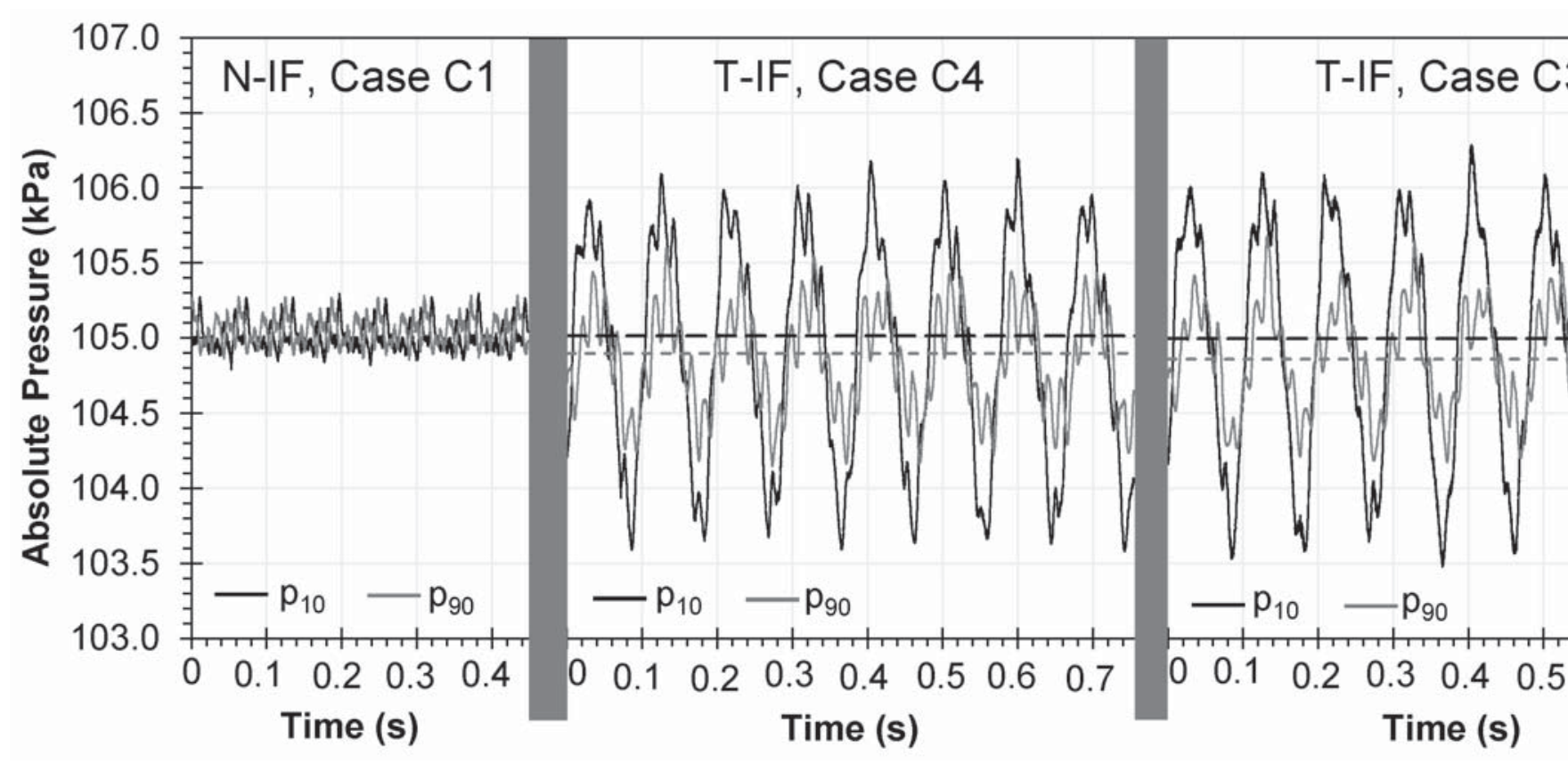

Figure 2.14. Time-varying absolute pressures at $x=10 \mathrm{~cm}$ and $x=90 \mathrm{~cm}$ without imposed fluctuations (N-IF) ar $f_{P: V}=10.6 \mathrm{~Hz}$. Ten dynamic data sets were averaged in the time-domain. The data shown are representative sub dashed lines of the same shade (black / gray) as their corresponding dynamic values. The dashed lines are not sh

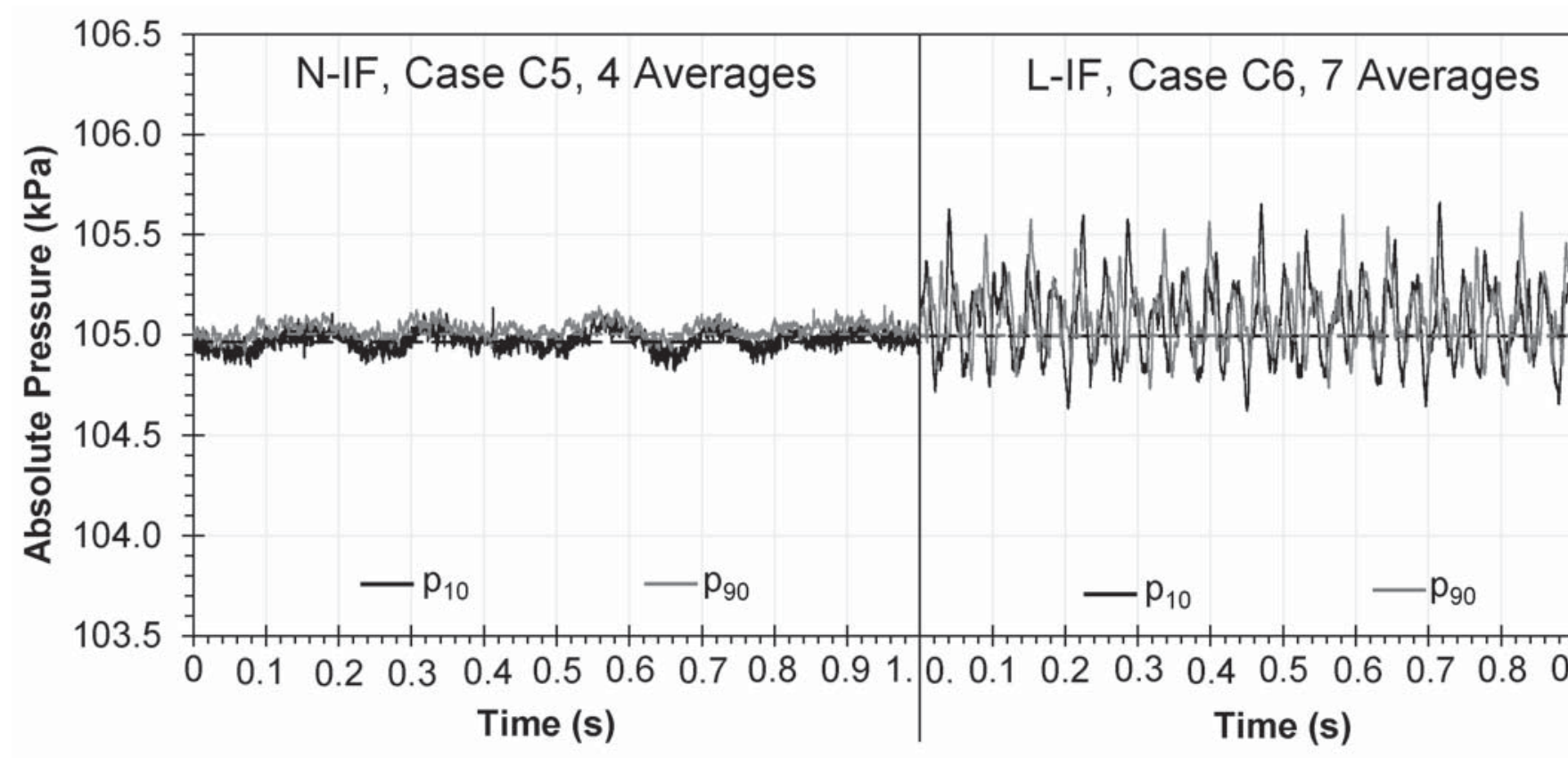

Figure 2.15. Time-varying absolute pressures at $x=10 \mathrm{~cm}$ and $x=90 \mathrm{~cm}$ without imposed fluctuations (N-IF) a $f_{P: V} \approx 28.4 \mathrm{~Hz}$. The number of dynamic data sets averaged in the time-domain for each case (run) is indicated in $\mathrm{t}$ given in Table 2.1 and shown here as horizontal dashed lines of the same shade (black or gray) as their correspor 

Figures $2.14-2.15$ show the nature of the imposed fluctuations through the test-section absolute pressures. Changes in long-term pressure values are much smaller than the amplitudes of the fluctuations. Note that the absolute pressure fluctuations in Fig. 2.14 are very similar in amplitude for runs $\mathrm{C} 4, \mathrm{C} 3$, and $\mathrm{C} 2$. This was due to the choices of settings for valve $\mathrm{V}_{\mathrm{P}}$ in Fig. 2.4; it does not represent a physical limitation of the equipment at the 10.6 Hz frequency. A larger range of absolute pressure fluctuations is demonstrated at the 28.4 Hz frequency through cases C6 and C10 in Fig. 2.15. Note that the level of the imposed pressure fluctuations has an effect on the long-term pressure-difference between $x=10 \mathrm{~cm}$ and $x=90 \mathrm{~cm}$ as shown by the varying gap between the dashed lines in Figs. $2.14-2.15$. The constancy of the long-term averages of the absolute pressure at $\mathrm{X}=10 \mathrm{~cm}$ is due to its use as the process variable for the PID feedback control of the liquid pump, the set point being $105.00 \mathrm{kPa}$ (note, as mentioned previously, that this does not mean that there will be no long-term excursions above or below this timeaveraged value, as may be seen in Figs. 2.7 and 2.10).

The flow seeks $\Delta T$ values consistent with the liquid pumping rate by varying the absolute pressure in the condenser, which varies the saturation temperature of the fluid subject to the fixed cooling condition defined in section 2.2.2.1. The hydrodynamics of the flow tend to require higher internal pressure drops between $x=10 \mathrm{~cm}$ and $x=90 \mathrm{~cm}$ for the T-IF and H-IF runs than for N-IF and L-IF runs. In Fig. 2.14, it is clear that a significant reduction in the amplitude of imposed absolute pressure fluctuations occurs between the $10 \mathrm{~cm}$ and $90 \mathrm{~cm}$ locations. To a lesser extent, this behavior may also be observed in Fig. 2.15 at the higher frequency. This suggests that the liquid film in the test-section was capable of absorbing a greater fraction of the acoustical energy supplied by the $10.6 \mathrm{~Hz}$ imposed fluctuations than that of the $28.4 \mathrm{~Hz}$ imposed fluctuations.

For the H-IF fluctuations at $10.6 \mathrm{~Hz}$ in Fig. 2.14, the nature of the temporal variations in absolute pressure at $x=90 \mathrm{~cm}$ bears significant differences from that near $x=10 \mathrm{~cm}$; specifically, there is a frequency near $62 \mathrm{~Hz}$ that persists in the absolute pressure response at $x=90 \mathrm{~cm}$. A similar frequency is also present at the $x=90 \mathrm{~cm}$ location for the other 
runs in Fig. 2.14, including the N-IF case $\mathrm{C} 1$. The amplitude at this frequency is less significant in the absolute pressure fluctuations at $x=10 \mathrm{~cm}$. This suggests that these pressure fluctuations are caused by something downstream of the test-section, such as the vanes of the compressor $C_{R}$ in Fig. 2.4. However, these fluctuations are much less significant for N-IF case C5 and H-IF case C10 in Fig. 2.15. It is unclear why this is the case, although it is possible that there might have been some liquid in the vapor sight glass (see Fig. 2.4) above the test-section exit which damped out the compressor-vaneinduced fluctuations for cases $\mathrm{C} 5$ and $\mathrm{C} 10$. Manual data and notes taken during the experiment do not address this issue, but it is known that for some of the experimental cases there was liquid in this vapor sight glass, and for others the vapor sight glass was dry except for an extremely thin condensing film on its interior surface. 


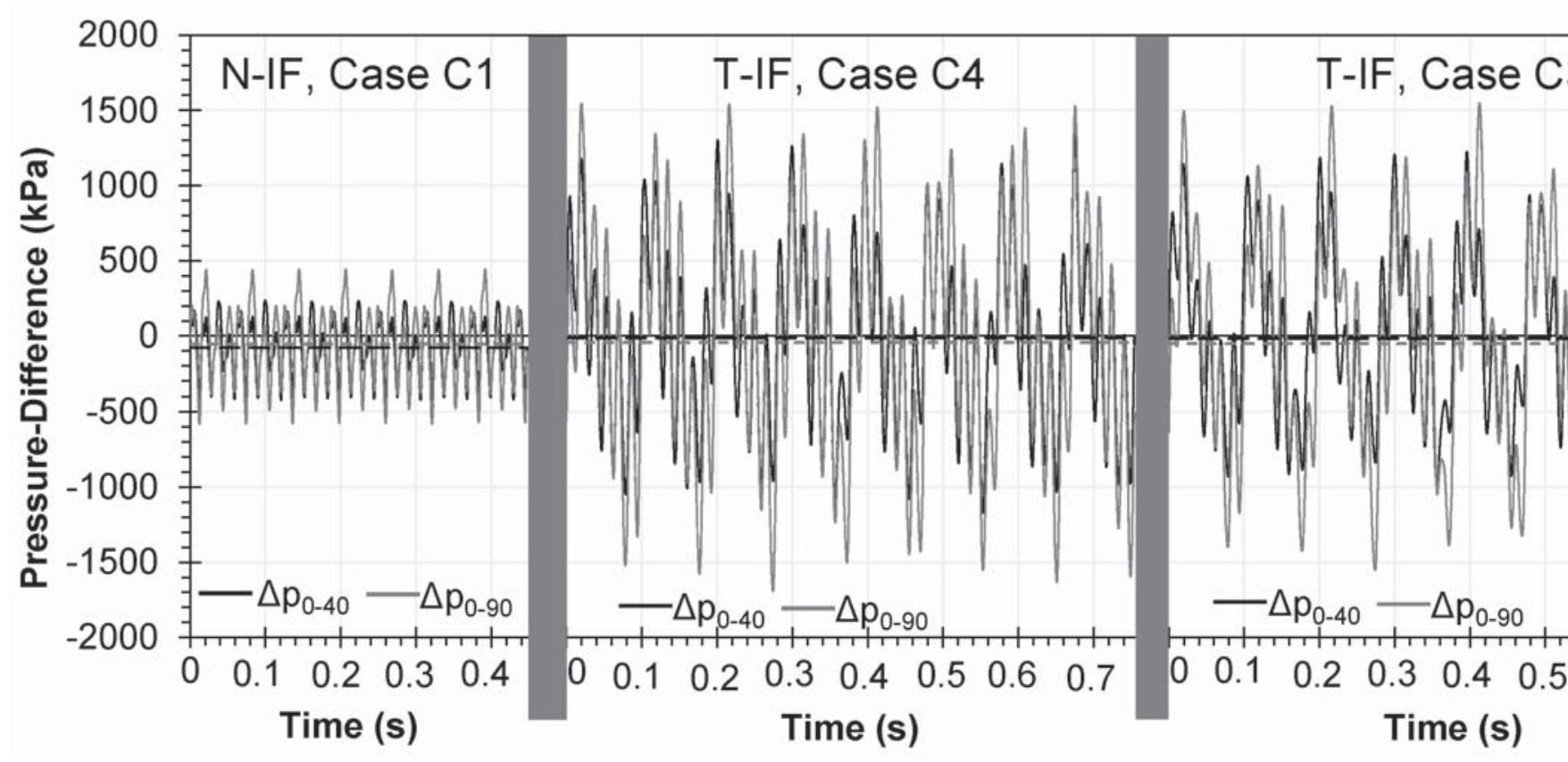

Figure 2.16. Time-varying pressure-differences over $0 \leq x \leq 40 \mathrm{~cm}$ and $0 \leq x \leq 90 \mathrm{~cm}$ without imposed fluctuatic right) at $f_{P: V}=10.6 \mathrm{~Hz}$. Ten dynamic data sets were averaged in the time-domain. The data shown are representa horizontal dashed lines of the same shade (black or gray) as their corresponding dynamic values.

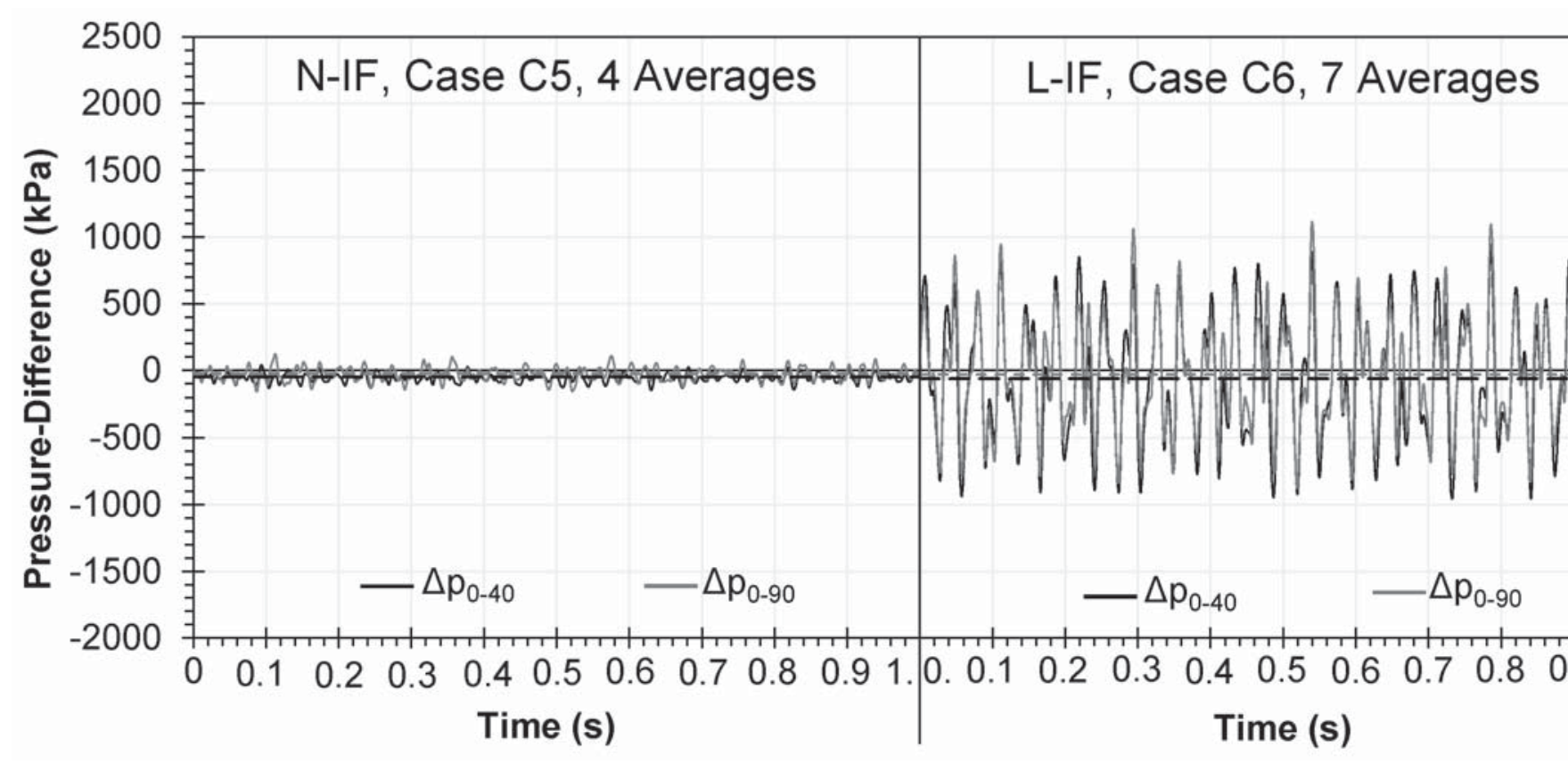

Figure 2.17. Time-varying pressure-differences over $0 \leq x \leq 40 \mathrm{~cm}$ and $0 \leq x \leq 90 \mathrm{~cm}$ without imposed fluctuatic at $f_{P: V} \approx 28.4 \mathrm{~Hz}$. The number of dynamic data sets averaged in the time-domain for each case (run) is indicated i are given in Table 2.1 and shown here as horizontal dashed lines of the same shade (black or gray) as their corres 

Figures $2.16-2.17$ show similar trends to Figs. $2.14-2.15$, but there is not as much reduction in the amplitude of fluctuations in pressure-difference for the $10.6 \mathrm{~Hz}$ runs of Fig. 2.16 of $\Delta p_{0-90}$ relative to $\Delta p_{0-40}$, compared with the reduction in amplitude of absolute pressure at $x=90 \mathrm{~cm}$ relative to the amplitude of absolute pressure at $\mathrm{X}=10 \mathrm{~cm}$ shown in Fig. 2.14 .

In Figures 2.18 - 2.19, long-term average values of $\mathrm{q}^{\prime \prime}{ }_{40}$ were taken from Table 2.1, and long-term average values of $\delta_{40}$ were computed using the arithmetic mean of $\delta_{40}(t)$ values from the previously described time-domain-averaged dynamic data block for each case (a portion of which is shown in the time-period given in Fig. 2.18 or 2.19).

The time-domain-averaged dynamic data blocks were obtained by the following process:

(i) group processed data blocks from individual dynamic data blocks within a case based on the number of pulsation cycles $\Delta t_{C}=1 / f_{p}$ and selecting the largest group of data blocks for additional analysis;

(ii) phase-shift the data for each of the individual dynamic data blocks within the group to match as closely as possible with a representative dynamic data block (generally the first block for the group), using a combination of trial-and-error and graphical analysis, with a "least squares" optimization criterion;

(iii) assign time-vectors to the individual phase-shifted data blocks within the group based on the sample interval 0.0005 seconds $\left(1 / f_{s}\right.$, where $f_{s}=2000 \mathrm{~Hz}$ is the sample frequency for the dynamic data) and a time between $0 \mathrm{~s}$ and $0.0005 \mathrm{~s}$ assigned to the first sample based on data processing parameters used to select the start and end of each block;

(iv) scale the time-vectors of the individual phase-shifted data blocks within the group by the ratio of their time-period (equal to the number of samples multiplied by the sample interval, generally between $3 \mathrm{~s}$ and $5 \mathrm{~s}$ ) to the average time-period for the group;

(v) up-sample — by means of linear interpolation - each phase-shifted data block within the group to provide samples for each time in the time-vectors of each of the 
other phase-shifted data blocks within the group (all of the resulting up-sampled data blocks now have the same values in their time-vectors);

(vi) average the up-sampled time-vectors to obtain the time-vector corresponding to the averaged response; and

(vii) average, for each of the relevant flow variables, the up-sampled vectors containing their values, to obtain their averaged response.

This method is used because small variations in the imposed pulsation frequency between dynamic data blocks (obtained for the same case) cause slight differences in the lengths of the processed dynamic data blocks within each case. The intent is to preserve all the relevant correlated frequency components through the averaging process with reduced distortion (from averaging) that is independent of time for the averaged data. Since each raw data value is represented once in the averages, absolute maximum and minimum vectors (not reported here) can be established for each relevant flow variable.

A much simpler alternative, which requires a small fraction of the computations required for the method stated above, is to "line up" the individual dynamic data block elements as nearly as possible to minimize the squares of the differences between data blocks for the relevant flow variables (due to phase misalignment), and average their values directly. However, at one or both ends of the dynamic data block, the frequency response of the averaged data block will contain distortion because correlated frequency components (of slightly different frequencies) will not remain in-phase throughout the length of the averaged block. The effect is compounded for harmonics of $f_{p}$, which have shorter time-periods.

The choice between these methods is an excercise in choosing the compromises one is willing to make in representing quasi-periodic data as if they were strictly periodic. A strictly periodic representation is desireable because it allows the long-term response of relevant flow variables to be represented by a much shorter time-period. Averaging between these shorter time-periods will eliminate uncorrelated frequency components (such as random noise from various sources). 


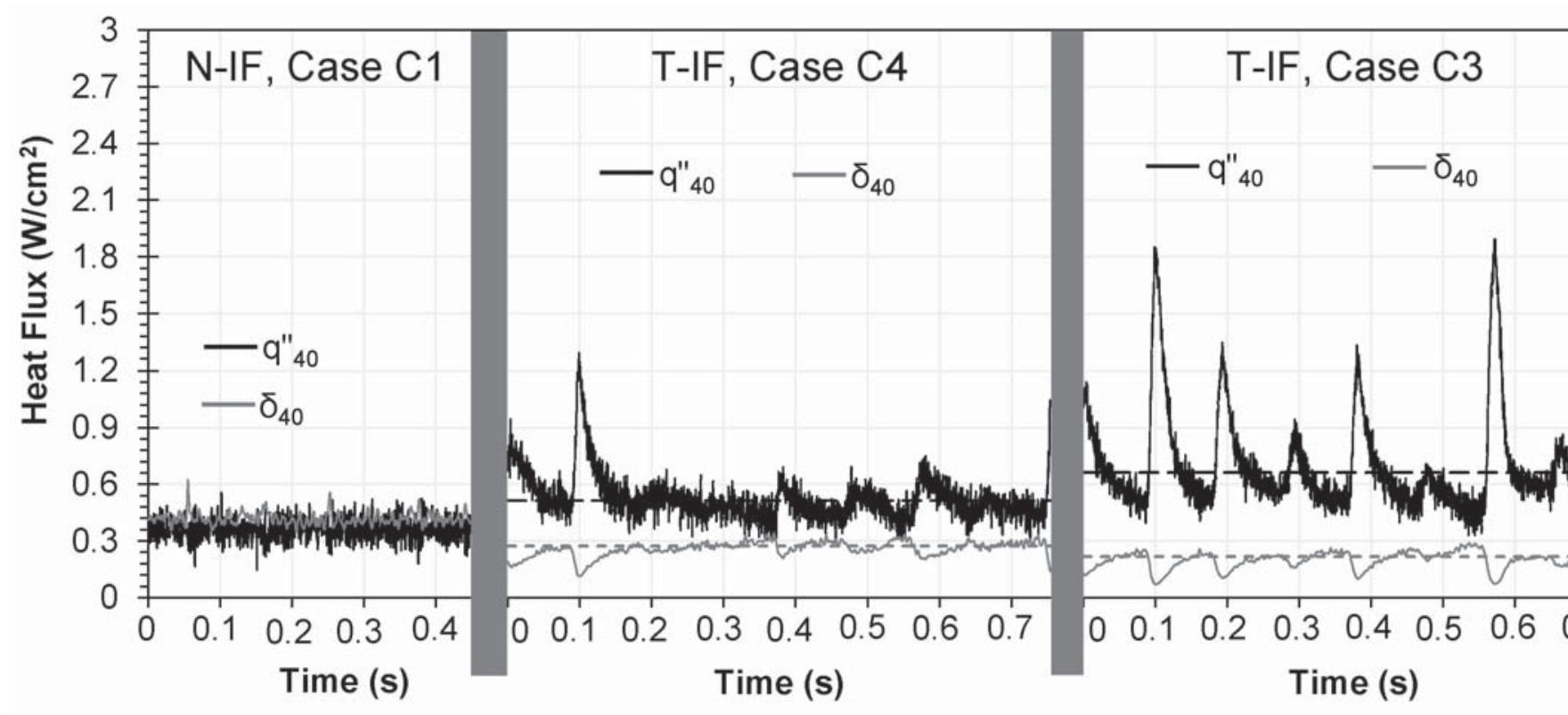

Figure 2.18. Time-varying measured heat-flux and calculated film thickness at the $x=40 \mathrm{~cm}$ location in the test-s each case (run) from left to right) at $f_{P: V}=10.6 \mathrm{~Hz}$. Ten dynamic data sets were averaged in the time-domain. The as horizontal dashed lines of the same shade (black or gray) as their corresponding dynamic values. Case C2 sho

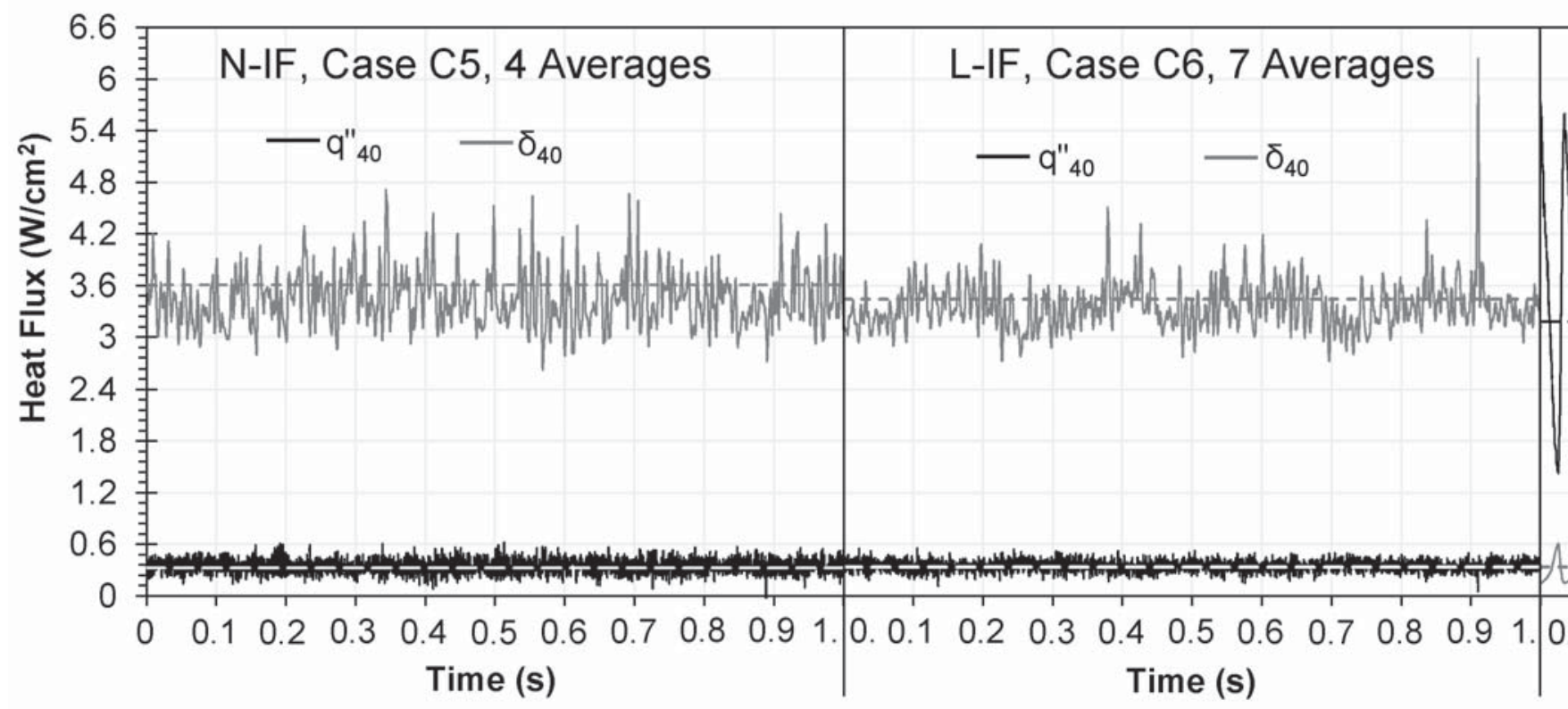

Figure 2.19. Time-varying measured heat-flux and calculated film thickness at the $x=40 \mathrm{~cm}$ location in the test-s each case (run) from left to right) at $f_{P: V} \approx 28.4 \mathrm{~Hz}$. The number of dynamic data sets averaged in the time-domair Table 2.2. The long-term average values are shown here as horizontal dashed lines of the same shade (black or $g$ as white dashed lines. Case $\mathrm{C} 10$ showed an $873 \%$ increase in long-term time-averaged heat-flux relative to case $-0.021 \mathrm{~W} / \mathrm{cm}^{2}$ at times $0.88876 \mathrm{~s}$ and $0.88902 \mathrm{~s}$, respectively. 

Figures $2.14-2.19$ clearly show that causes underlying the changes in the mean values of $\Delta p_{0-40}$ and heat-flux $q^{\prime \prime}{ }_{40}$ are dynamic in nature and are associated with pulsations (at the primary imposed frequency of $10.6 \mathrm{~Hz}$ for Figs. 2.14, 2.16, and 2.18 or $28.4 \mathrm{~Hz}$ for Figs. 2.15, 2.17, and 2.19). The FFTs of these two variables, sampled in Fig. 2.20 for cases $\mathrm{C} 5$ and $\mathrm{C} 10$ (28.4 Hz primary imposed frequency), clearly show the impact of the dominant frequency. Data points at significant peaks in the amplitude spectra of Fig. 2.20 have been highlighted, with hollow points used for case C5 and solid points used for case $\mathrm{C} 10$. The mean values at $\mathrm{f}=0 \mathrm{~Hz}$ are indicated by the data points on the vertical axes. The highest peak in the $\mathrm{C} 10$ data corresponds to the frequency of imposed vapor pulsations.

There are two significant harmonics of this peak (fundamental frequency) in the pressure-difference and three significant harmonics of this peak in the heat-flux. These harmonics indicate that the shape of the time-variations in the original signal is non-sinusoidal, as may be observed in Figs. 2.17 and 2.19 (note variations in the peak locations of the H-IF fluctuations). The highest non-zero peaks (at $32.97 \mathrm{~Hz}$ and its multiple $65.94 \mathrm{~Hz}$ ) of the $\Delta \mathrm{p}_{0-40}$ values for case C5 are relatively small compared to those of case $\mathrm{C} 10$ but are significant compared to their mean for case C5. These probably come from flow fluctuations induced by other sources (e.g. the vanes of the compressor, etc.). The pressure-difference for case $\mathrm{C} 10$ also shows two more significant peaks not directly related to the imposed pulsations at $64.39 \mathrm{~Hz}$ and $80.53 \mathrm{~Hz}$. The $64.39 \mathrm{~Hz}$ pressure-difference fluctuation for case $\mathrm{C} 10$ probably comes from the same source as the 65.94 Hz pressure-difference fluctuation for case C5. There is a smaller pressure-difference fluctuation for case $\mathrm{C} 10$ around $32 \mathrm{~Hz}$, which might correspond to the $32.97 \mathrm{~Hz}$ fluctuation for case $\mathrm{C}$. The source of the $80.53 \mathrm{~Hz}$ pressure-difference fluctuation for case $\mathrm{C} 10$ may be due to a factor (such as a resonance related to the liquid-vapor interfacial waves, the test-section mounting hardware, or the differential pressure transducer DPT-1 diaphragm) which is excited by the second harmonic of the imposed pulsation frequency at around $85.2 \mathrm{~Hz}$. 
Possible sources of smaller FFT magnitudes associated with pressure-difference and heat-flux curves in Fig. 2.20 include turbulent eddies in the vapor flow, effects of condensing surface vibrations, electromagnetic interference, and other noise introduced by the electronics of the sensors and heat-flux meter amplifier.

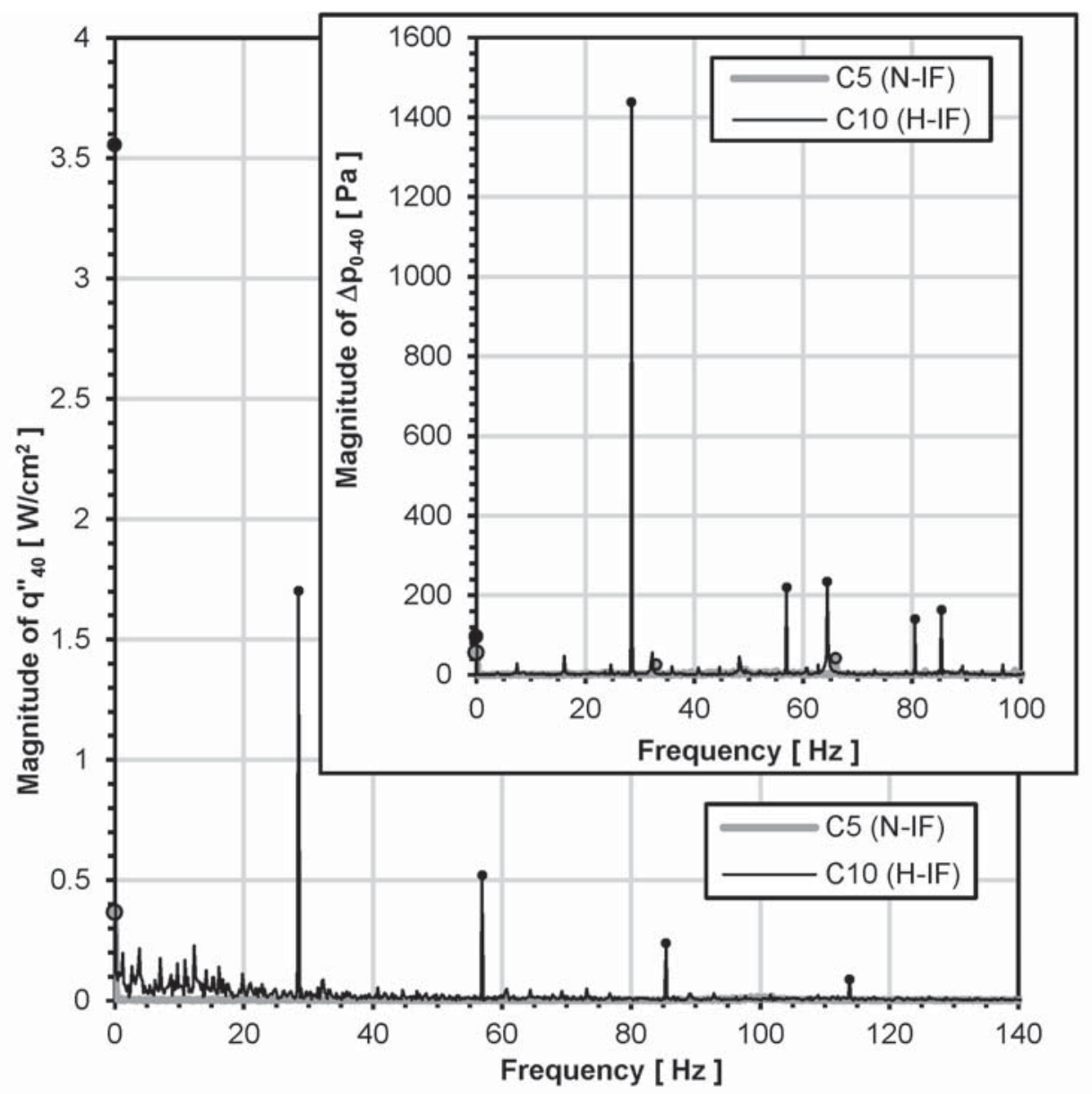

Figure 2.20. Fourier transform magnitude plots for $40 \mathrm{~cm}$ heat flux and $0-40 \mathrm{~cm}$ pressure-difference in partial condensation. Significant peaks are marked with grey-filled circles for case C5 and solid black dots for case C10.

The question arises as to why the interfacial waves generated by the pulsations cause the annular pulsatile film flows (confirmed by visual observations, see representative photograph in Fig. 2.1b and Videos 2-1 - 2-2) to change the mean values of variables (e.g. local heat-flux at the $40 \mathrm{~cm}$ location) in a certain direction (e.g. mean local 
heat-flux increased by $864 \%$ in Fig. 2.18).

To provide a rough understanding of the underlying reasons, and in the absence of dynamic film thickness measurements at the $x=40 \mathrm{~cm}$ location, the dynamic film thickness results shown in Figs. 2.18 - 2.19 are helpful. These film thickness values were calculated using the equation $\delta_{40} \equiv \mathrm{k}_{\mathrm{L}} \cdot\left(\mathrm{T}_{\text {Sat }}-\mathrm{T}_{40}\right) / \mathrm{q}^{\prime \prime}{ }_{40}$, where $\mathrm{k}_{\mathrm{L}}$ is the thermal conductivity of the liquid FC-72 calculated at the average of $\mathrm{T}_{\text {Sat }}$ and $\mathrm{T}_{40}$. This calculation depends on the assumption that conduction is the only mode of heat transfer through the liquid film and that equilibrium thermodynamics applies at the interface between the liquid and vapor phases. For the pulsatile case C10, the dynamic film thickness values at the wave-troughs in Fig. 2.18 are the smallest possible film thickness values because they have been calculated using the molecular conductivity value, $\mathrm{k}_{\mathrm{L}}$, which should be replaced by an effective thermal conductivity, $\mathrm{k}_{\text {eff-L }}$ (which, unlike its definition for turbulent flows (see p. 256 in [26]), is being introduced to take into account the instantaneous convective effects due to laminar eddies in the vicinity of the wave-trough locations). This is important because, at this location, the transverse components of liquid velocity are comparable to the axial components in the flow-direction. Because of this, depending on the nature of pulsatile flow conditions, it is expected that:

$\mathrm{k}_{\text {eff-L }} \sim \mathrm{k}_{\mathrm{L}}, \mathrm{k}_{\text {eff-L }}>\mathrm{k}_{\mathrm{L}}$, or $\mathrm{k}_{\text {eff- } \mathrm{L}}>>\mathrm{k}_{\mathrm{L}}$.

Note that several accurate steady/unsteady continuum-level simulations for condensing flows, see [21 - 24], support the assumption (used in Fig. 2.18) that fluid flow at the thin wave-troughs is laminar (local film Reynolds numbers are small) and instantaneous heat flow through the dynamic micro-meter thin condensate is driven by linear instantaneous temperature profiles. However, in the presence of adsorbed and micro-layer phenomena (as discussed below in section 2.3.5) and vigorous laminar eddy motions (with comparable axial and transverse velocities), appropriate modeling of the flow physics is likely to yield the result that the linearity of temperature profile may still be valid with significant effects of micro-layer convection. 
Estimates shown in Fig. 2.19 for $\mathrm{f}_{\mathrm{P}: \mathrm{V}} \approx 28.4 \mathrm{~Hz}$ clearly suggest that both the troughs and the crests of the film remain within $20-70 \mu \mathrm{m}$. This means that when wave-troughs cause the liquid-vapor interface to dynamically approach the wetting surface (to within $30 \mu \mathrm{m}$ ), some new physics prohibits the wave-crests from returning towards the original $300 \mu \mathrm{m}$ thick film associated with the thick N-IF case. New physics is suspected because the typical wave asymmetries under continuum modeling simulations [21 - 24] may at most lead to $10-40 \%$ heat-flux enhancements. It should be noted that dramatic heat-flux enhancement behavior was also repeatedly observed for thin and wavy films in the annular regions of pulsatile fully condensing flows (see [13 - 14] and chapter 3). However, unlike dynamic behavior in Fig. 2.19, the dynamic film in Fig. 3.16 (Fig. 13 of [14]) showed wave crests (order $200 \mu \mathrm{m}$ ) that were significantly higher than the wave troughs (order $20 \mu \mathrm{m}$ ) and were closer to their corresponding average N-IF value (approximately $260 \mu \mathrm{m}$ ).

The behavior of the H-IF pulsatile innovative condensing flow of Fig. 2.18 for case C2 at $f_{P: V}=10.6 \mathrm{~Hz}$ showed a more similar response to the completely condensing flows of chapter 3 or [13 - 14]. In Fig. 2.18, it is clear that the time-varying film thickness for case $\mathrm{C} 2$ spends more time in the wave peaks than in the wave troughs, but the time-varying film thickness for case C10 in Fig. 2.19 spends more time in the wave troughs than in the wave peaks. Reasons for this are discussed later.

The increase in the mean (time-averaged) heat-flux with pulsation amplitude (as measured by the amplitude of $\mathrm{p}_{10}$ or $\Delta \mathrm{p}_{0-40}$ ) at the above considered $10.6 \mathrm{~Hz}$ and 28.4 Hz primary frequencies is systematically observed in the experimental runs, and this fact is clearly seen in Fig. 2.21.

Note that the two T-IF cases C4 and C3 of Fig. 2.18 represent the upper and lower bounds of the transition region within which the heat-flux enhancement becomes significant. These points are indicated by the middle two gray squares in the series for the average response at the $10.6 \mathrm{~Hz}$ imposed fluctuation frequency. Also note that these 
time-averaged heat-flux values are similar to the time-averaged heat-flux value for case C7 (also labeled as T-IF in Tables $2.1-2.2$ ), indicated by the black ' $X$ ' behind the green circles in Fig. 2.21. This shows that there is a well-defined range of heat-flux values at which pulsation-induced enhancement effects become significant, and that this range has minimal dependence on frequency for the innovative imposed fluctuation flows investigated here.

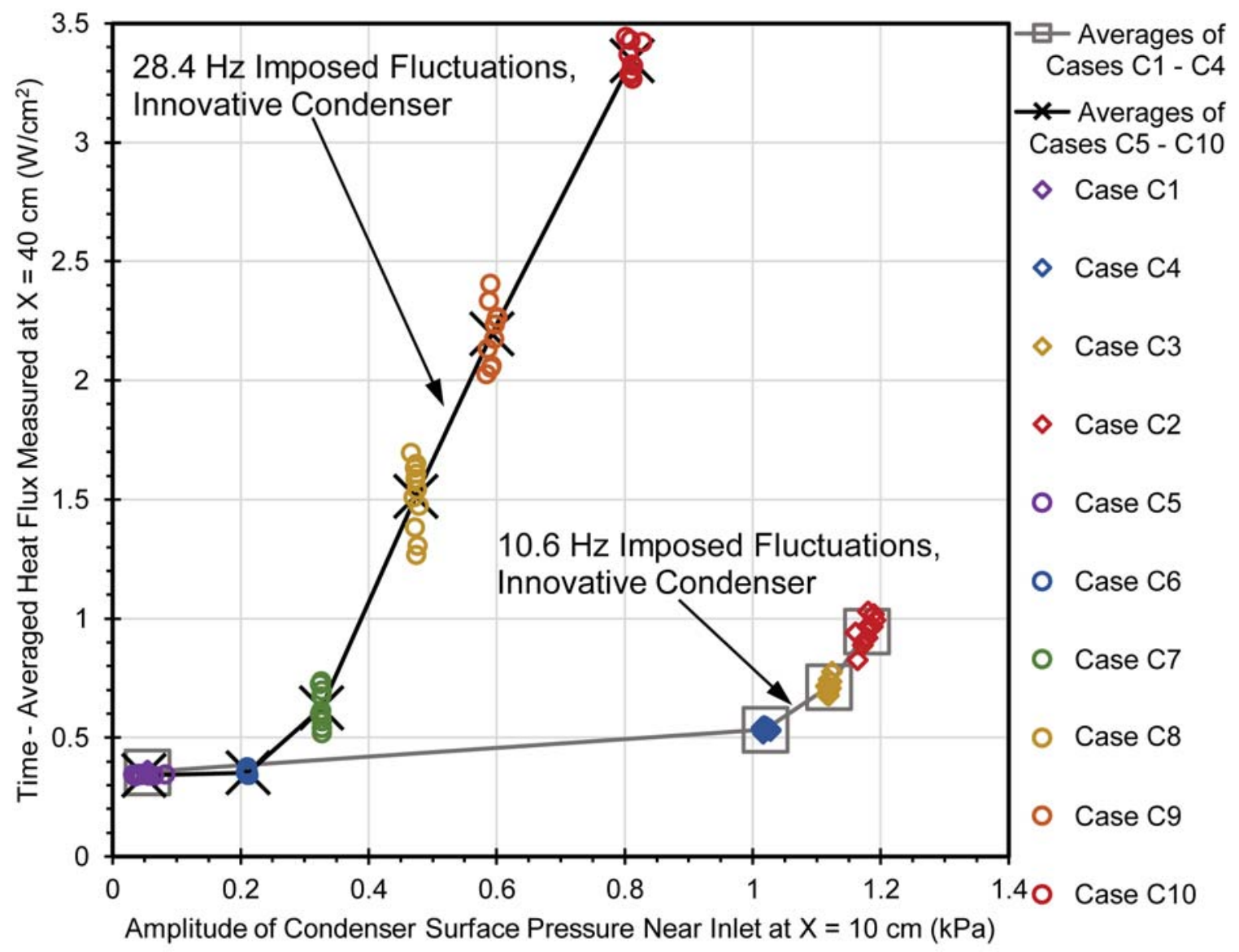

Figure 2.21. Innovative condensing flow heat-flux enhancement chart with respect to amplitude of absolute pressure fluctuations at $x=10 \mathrm{~cm}$. Note that the $10.6 \mathrm{~Hz}$ imposed fluctuations require higher amplitude upstream absolute pressure fluctuations to achieve the same levels of heat-flux enhancement.

Figure 2.21 also shows remarkable frequency dependence in the heat-flux enhancement behavior regarding the levels of inlet pressure fluctuation amplitudes that are necessary 
to induce significant heat-flux enhancement. For example, over the pulsation amplitudes considered, $f_{P}=10.6 \mathrm{~Hz}$ imposed pulsations do not show enhancement trends that are as significant as for $\mathrm{f}_{\mathrm{P}}=28.3 \mathrm{~Hz}$. This is in contrast with pulsatile annular flows associated with pulsatile fully condensing flows in Fig. 3.15 (see Fig. 12 in [14]), where there were significant enhancement trends over the entire range of frequencies considered $(3.9-16.2 \mathrm{~Hz})$, especially in the $10.2-16.2 \mathrm{~Hz}$ range. Since fully condensing flows provide an effective oscillatory liquid plug at the end of the annular regime ([14] and section 3.4.5), the wave amplitudes are larger (and wave-troughs closer to the wetting surface) for a range of frequencies (e.g. 3.5-20 Hz). Such desirable frequency response behavior from fully condensing flows in chapter 3 (or [14]) may be achieved for the innovative condenser operations in Fig. 2.1b if exit film thickness is controlled and the rigid end wall is replaced by a suitably designed end plate which vibrates (under near-resonant conditions) due to pressure pulsations associated with the pulsatile flows.

Figure 2.22 is analogous to Figure 2.21, except that the time-averaged heat flux at the $x=40 \mathrm{~cm}$ location is plotted vs. the amplitude of pressure-difference over $0 \leq x \leq 40 \mathrm{~cm}, \Delta p_{0-40}$, instead of vs. the amplitude of absolute pressure at $x=10 \mathrm{~cm}$, $\mathrm{p}_{10}$, at imposed pulsation frequency $\mathrm{f}_{\mathrm{P}: \mathrm{V}}$. Note that the frequency dependence is not as strong for Fig. 2.22 as for Fig. 2.21, and the response is reversed - the lower frequency pulsations (at $10.6 \mathrm{~Hz}$ ) realize heat-flux enhancement at lower amplitudes of $\Delta \mathrm{p}_{0-40}$ than the higher frequency pulsations (at approximately $28.4 \mathrm{~Hz}$ ). The amplitudes of $\Delta p_{0-40}$ may be considered to indirectly correlate with the amplitudes of volume flow rates (and by extension mass flow rates because $\rho_{\mathrm{v}}$ can be considered nearly constant for these low Mach number flows) averaged over $0 \leq x \leq 40 \mathrm{~cm}$. This may suggest that it is the flow rate fluctuations which control the heat-flux enhancement phenomenon, and the pressure fluctuations are related by hydrodynamics.

Following this line of reasoning, the fully condensing flows of chapter 3 were more effective at converting absolute pressure fluctuations near the test-section inlet into flow fluctuations at the $x=40 \mathrm{~cm}$ location because of a "spring" effect caused by the 
compressibility of the confined vapor pockets in the plug/slug and bubbly flow regimes. As previously mentioned, the use of an active flow element (such as a passively vibrating plate exposed to the vapor flow) near the exit of the annular flow condenser is expected to be able to provide effectiveness similar to or better than the non-annular flow regimes provide for completely condensing flows - in allowing higher amplitude flow rate pulsations (with respect to amplitudes of absolute pressure pulsations supplied to the condenser) over a large range of imposed pulsation frequencies.

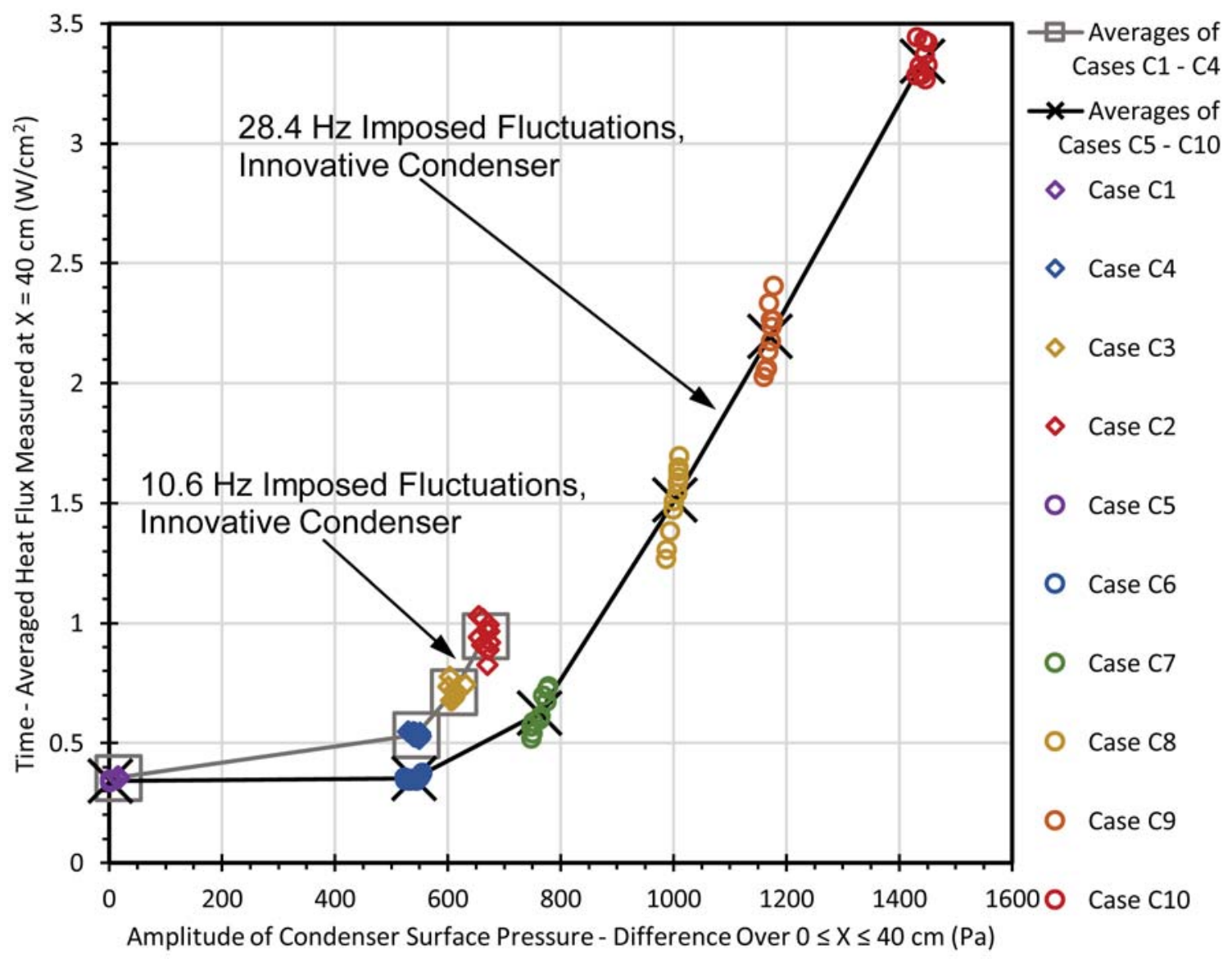

Figure 2.22. Innovative condensing flow heat-flux enhancement chart relative to pressure-difference fluctuations over $0 \leq x \leq 40 \mathrm{~cm}$. Note that the $10.6 \mathrm{~Hz}$ imposed fluctuations require lower amplitude $0-40 \mathrm{~cm}$ pressure-difference fluctuations to achieve the same levels of heat-flux enhancement.

Figure 2.23 shows time-averaged heat-flux vs. amplitude of estimated absolute pressure 
at $x=40 \mathrm{~cm}, p_{40}$. The value of $p_{40}$ was calculated from the difference $p_{40}=p_{0}-\Delta p_{0-40}$, where $\mathrm{p}_{0}$ was taken from an absolute pressure transducer connected to a liquid pressure tap (not shown in Fig. 2.3c) underneath the stainless steel plate of Fig. 2.3c at the $x=0 \mathrm{~cm}$ location. The values of $p_{40}$ calculated this way were not accurate enough to be useful, but their amplitudes calculated through their FFTs are accurate enough to allow analysis (see Fig. 2.24).

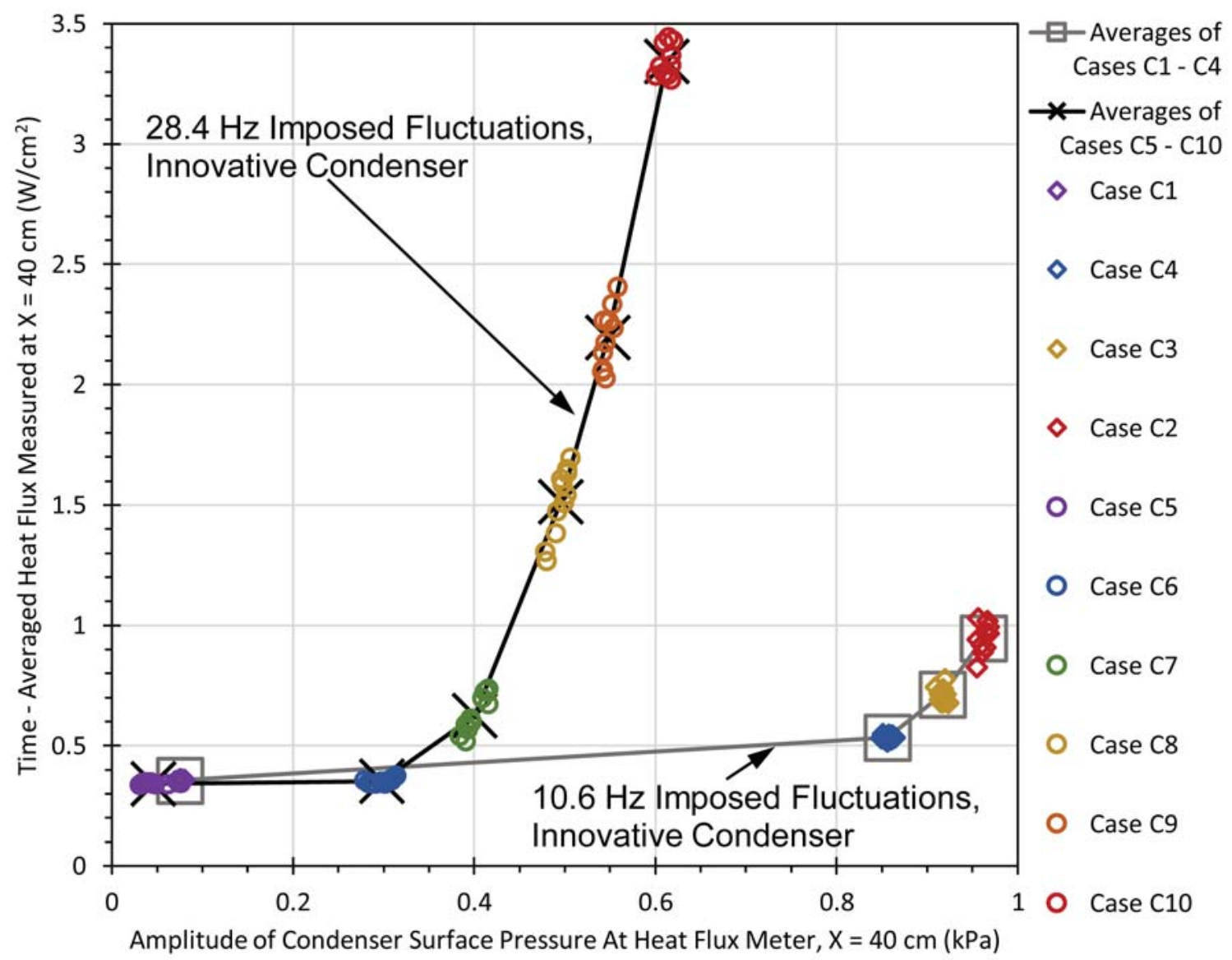

Figure 2.23. Innovative condensing flow heat-flux enhancement chart relative to absolute pressure fluctuations estimated at $x=40 \mathrm{~cm}$. Note that the individual data points for the $28.4 \mathrm{~Hz}$ imposed fluctuations fall along the same curve as the averaged response.

Note that the individual data points match the time-averaged response much better than in Figs. $2.21-2.22$ for the $28.4 \mathrm{~Hz}$ imposed pressure fluctuations. This is because the absolute pressure fluctuation amplitude and time-averaged heat-flux are being taken from 
the same location. The variations in the data points for each case at the $10.6 \mathrm{~Hz}$ imposed pressure fluctuations are not spread out enough to determine how well the individual data points follow the averaged response (their variations are within measurement accuracy limits).

Figure 2.24 shows the variations of absolute pressure amplitudes along the length of the test-section for cases $\mathrm{C} 1-\mathrm{C} 10$. The amplitudes at $\mathrm{x}=0 \mathrm{~cm}, \mathrm{x}=10 \mathrm{~cm}, \mathrm{x}=90 \mathrm{~cm}$, and $x=100 \mathrm{~cm}$ were measured by absolute pressure transducers. The amplitudes at $x=40 \mathrm{~cm}$ were calculated as previously described.

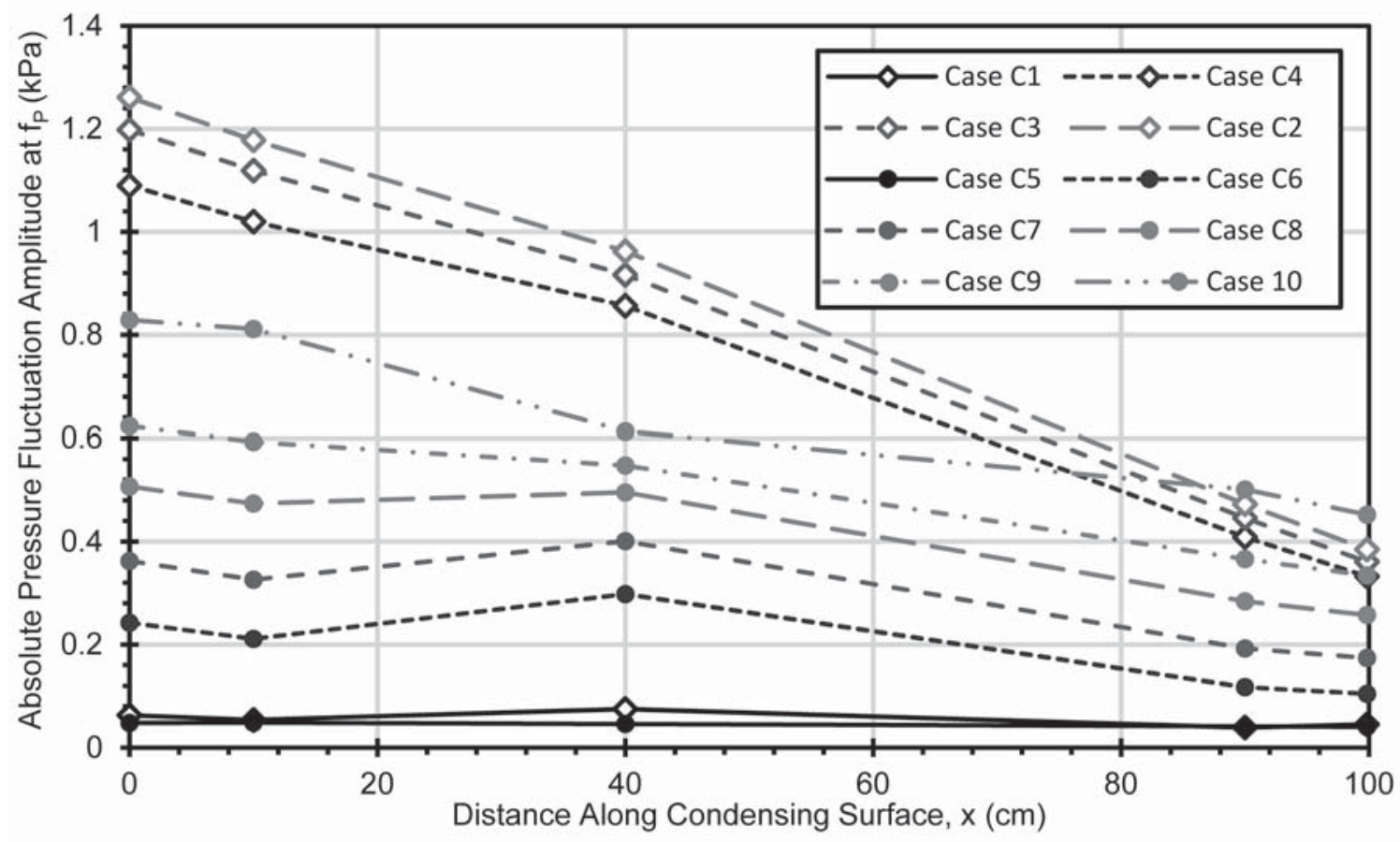

Figure 2.24. Variation of absolute pressure fluctuation amplitudes along the length of the condenser. Cases (runs) C2 - C4 represent imposed fluctuations at $10.6 \mathrm{~Hz}$, cases C6 - C10 represent imposed fluctuations at approximately $28.4 \mathrm{~Hz}$, and cases $\mathrm{C} 1$ and $\mathrm{C} 5$ represent no-imposed fluctuations (N-IF) cases. Note that the trend is toward reduction in amplitude as the pulsations move downstream, and some of their energy is absorbed by the liquid film.

Although dynamic flow rate measurements within the test-section were not available for the experiments reported here, it is expected, based on Fig. 2.22, that if one could 
create a graph of time-averaged heat flux at $x=40 \mathrm{~cm}$ vs. amplitude of volume flow rate fluctuations at $x=40 \mathrm{~cm}$, it would show the reduced frequency dependence of Fig. 2.22 along with the trends of individual dynamic data points matching their averaged response as in Fig. 2.23. If future experiments establish this, it would imply that the level of heat-flux enhancement at a given location depends directly on the amplitudes of the time-variations of the velocity profile at that location (considering the dependence of instantaneous cross-sectional flow rates on cross-sectionally averaged local velocity profiles). The local velocity gradients in the vapor phase near the liquid-vapor interface at a particular location within the condenser are the direct cause of the interfacial shear-stresses at that location, which drive the local liquid flow rates and the interfacial morphology for these horizontal annular condensing flows. Although this correlation between velocity profile variation amplitudes in time and the observed heat-flux enhancement phenomenon would describe the mechanics of the heat-flux enhancement phenomenon, it would not explain the underlying physical principles or provide the underlying cause of this phenomenon. Following is an attempt at explaining the underlying physical principles, and thereby answering the question as to why the enhancement phenomenon occurs to such an extraordinary degree.

All the above observations are consistent with the fact that small (in tens of $\mu \mathrm{m}$ ) film thickness values at wave-troughs are required, and this is only facilitated over a longer condenser length if the forward moving wave amplitudes (due to inlet vapor pulsations) are augmented by standing interfacial waves generated through right exit conditions (e.g. liquid pressure, liquid thickness, geometry, etc.).

Clearly right wave-amplitude augmentation conditions existed (up to the $x=40 \mathrm{~cm}$ location) for the cases where significant enhancements were observed. In particular, the presence of two mechanisms - interfacial wave retardation/reflection from downstream exit locations and superposed interfacial waves associated with pulsatile acoustic wave-energy in the annular vapor phase portions (as achieved under pulsatile inlet vapor flow rate conditions of chapter 3, or [14]) — are probably responsible for a somewhat 
frequency-independent behavior (within $3.9-16.2 \mathrm{~Hz}$ ) in chapter 3 and [14] as opposed to the more pronounced frequency dependent behavior in Fig. 2.21.

Also, as far as results in Figs. 2.21 - 2.23 are concerned, the reflection of vapor phase acoustic waves from the exit and its coupling to interfacial waves appears to be important. The large amplitude annular flow realizations of heat-flux enhancement are significantly dependent on the exit condition (i.e. exit liquid film thickness, ability to impose different exit liquid pressures by independent means, existence or absence of a significant volume of stagnant vapor near the condenser exit, etc.) as well as the imposed frequency.

Steady-in-the-mean pulsatile flows show exit-condition dependency (or "elliptic" sensitivity) because time-average flow variables are being computed over several minutes, and steady-in-the-mean behaviors of relevant flow variables are influenced by the backward-and-forward moving interfacial waves over shorter time-scales

\subsubsection{Results specific to boiling flows realized in the innovative arrangement of Fig. $2.2 \mathrm{~b}$}

For the innovative boiling cases, the peristaltic pump $\mathrm{P}_{1}$ (see Fig. 2.5) introduced significant amplitude pulsations in the liquid flow rate supplied to the inlet of the test-section, which significantly influenced the time-variations in flow variables. Therefore, for the data reported in Table 2.3, two predominant pulsation frequency values are important — one associated with the imposed vapor pulsations supplied to the inlet of the test-section, and the other associated with the liquid pulsations arising from the rollers of the peristaltic liquid pump. For cases B1 - B10, the vapor pulsation frequency $f_{P: V}$ was chosen to nearly coincide with the liquid pulsation frequency $f_{P: L}$, so only one frequency $f_{P}$ and its corresponding amplitude values are reported for these cases in Table 2.3. 

Table 2.3.

Selected flow variables from innovative flow boiler cases.

\begin{tabular}{|c|c|c|c|c|c|c|c|c|c|c|c|c|c|}
\hline & $\mathbf{f}_{\mathrm{P}}$ & $p_{10}$ & 10 & $\dot{\mathbf{m}}_{\mathrm{I}: \mathrm{v}}$ & $\overline{\dot{m}_{\mathrm{I}: \mathrm{L}}}$ & $\mathrm{v}_{\mathrm{l}: \mathrm{V}}$ & $\overline{v_{\mathrm{l}: \mathrm{L}}}$ & $v_{E: V}$ & $\overline{T_{0: V}}$ & $T_{0: L}$ & $\Delta \mathrm{T}$ & $\mathrm{T}_{\text {Sat }}$ \\
\hline & & \pm 0.12 & $\pm 0.5+2 \%$ & $\pm 6 \%$ & $0.35 \%$ & $\pm 0.1 \%$ & \pm 0.28 & \pm 0.15 & \pm 0.36 & \pm 1 & \pm 1 & 0.32 & \pm 0.11 \\
\hline Run & Type & {$[\mathrm{Hz}]$} & [ $\mathrm{kPa}]$ & {$[\mathrm{kPa}]$} & {$[\mathrm{g} / \mathrm{s}]$} & {$[\mathrm{g} / \mathrm{s}]$} & {$[\mathrm{m} / \mathrm{s}]$} & [ $\mathrm{m} / \mathrm{s}$ ] & {$[\mathrm{m} / \mathrm{s}]$} & {$\left[{ }^{\circ} \mathrm{C}\right]$} & {$\left[{ }^{\circ} \mathrm{C}\right]$} & {$\left[{ }^{\circ} \mathrm{C}\right]$} & {$\left[{ }^{\circ} \mathrm{C}\right]$} \\
\hline B1 & N-IF & 3.02 & 120.00 & 0.327 & 299 & .585 & 3.64 & 0.52 & 8.58 & 75.94 & 31.40 & 7.77 & 61.22 \\
\hline B2 & H-IF & 3.00 & 03 & 168 & 00 & 49 & 3.03 & 5 & 42 & 71.74 & 32.82 & .42 & 1.43 \\
\hline B3 & H-IF & 3.00 & & 3.016 & 1.700 & 2.540 & 4.75 & 01 & 9.36 & 74.39 & 32.98 & .57 & 61.27 \\
\hline B4 & & 2.98 & & & & & & & 0 & 44 & 50 & & 3 \\
\hline B5 & H-IF & 2.41 & & & & & & & 58 & .54 & 31.39 & & .41 \\
\hline B6 & N-IF & 2.36 & & 0.312 & & 2.015 & 2.80 & & 6.54 & 71.56 & 31.21 & 10.01 & 61.49 \\
\hline B7 & N-IF & 3.89 & 47 & 0.292 & 1.804 & 2.939 & 5.76 & .59 & 12.44 & 65.02 & 27.48 & 6.69 & 56.49 \\
\hline B8 & H-IF & 3.79 & 97 & 2.783 & 1.852 & 2.977 & 5.78 & 59 & 12.24 & 63.66 & 25.27 & 4.27 & 57.36 \\
\hline B9 & T-IF & 3.79 & .79 & 1.171 & 1.834 & 2.970 & 5.74 & 59 & 12.31 & 62.30 & 23.59 & 86 & 57.17 \\
\hline $\mathbf{B 1 0}$ & L-IF & 3.80 & 106.46 & 0.637 & 1.823 & 2.965 & 5.72 & 0.59 & 12.31 & 62.37 & 23.93 & 2.89 & 57.07 \\
\hline
\end{tabular}



For innovative boiling cases, the total inlet mass flow rate is composed of independent liquid and vapor components, which are denoted as $\dot{m}_{1: L}$ and $\dot{m}_{1: V}$ respectively. Quasi-steady or steady-in-the-mean (over sufficiently long time periods) conditions exist for the reported data. If one were to use the symbol $\dot{m}_{\mathrm{I}: A C}$ to represent the mass flow rate of vapor supplied to the auxiliary condenser of Fig. 2.5, the conservation of mass at two T-junction locations (one downstream of the "vapor sight glass" above the test-section exit and the other downstream of the liquid pumps $P_{1}$ and $P_{2}$ ) implies: $\dot{\mathrm{m}}_{\mathrm{I}: \mathrm{V}}=\dot{\mathrm{m}}_{\mathrm{R}: \mathrm{V}}=\dot{\mathrm{m}}_{\mathrm{E}: \mathrm{V}}$ $-\dot{m}_{A C}$, and $\dot{m}_{\mathrm{l}: L}=\dot{m}_{A C}+\dot{m}_{E: L}$. For all of the cases reported in this paper, the incoming liquid was fully boiled off and the exit liquid flow rate from the boiler was zero or negligible, so $\dot{m}_{\mathrm{I}: \mathrm{V}}+\dot{\mathrm{m}}_{\mathrm{l}: \mathrm{L}}=\dot{\mathrm{m}}_{\mathrm{E}: \mathrm{V}}$ under the conditions of interest. Average velocity values $v_{\mathrm{l}: \mathrm{V}}, \mathrm{v}_{\mathrm{l}: \mathrm{L}}$, and $\mathrm{v}_{\mathrm{E}: \mathrm{V}}$ were obtained by the equation $\mathrm{v}_{\text {avg }}=\dot{m} /(\rho \cdot A)$, where 'A' represents the relevant cross-sectional area of the flow. In this case the average inlet liquid velocity is approximately $10-15 \%$ of the average inlet vapor velocity, and the average exit vapor velocity is $1.97-2.36$ times the average inlet vapor velocity. The resulting acceleration of the vapor further increases the pressure-differences along the length of the test-section. This is in contrast to the vapor deceleration effects on the pressure-differences for the innovative condensing flow cases.

The saturation temperature of the vapor, $T_{\text {Sat|Avg }}=T_{\text {Sat }}\left(\bar{p}_{\text {mean }}\right)$, is computed at the arithmetic mean of $\mathrm{p}_{10}$ and $\mathrm{p}_{90}, \overline{\mathrm{p}}_{\text {mean }}=\left(\overline{\mathrm{p}}_{10}+\overline{\mathrm{p}}_{90}\right) / 2$, due to the significant variation of pressure along the length of the test-section. The inlet liquid temperature, $T_{0: L}$, is reported because the warming of the liquid to its saturation temperature, as discussed next, is a significant percentage of the total heat transfer within the boiler. In this paper's boiling flow cases, there is a significant difference between inlet liquid temperature $T_{0: L}$ and the saturation temperature $T_{\text {Sat|Avg }}$. As a result, there is noticeable bubble nucleation over the first $10-15 \mathrm{~cm}$ of the annular boiling flow before it gets suppressed. Also, for the N-IF cases, the annular region over the first $20-30 \mathrm{~cm}$ of the boiling flow shows entrainment of some liquid drops in the vapor phase (for H-IF cases, the length over which entrainment occurs increases, and the appearance of the entrained droplets becomes intermittent). 
The nucleating bubble phenomena in the inlet zone is more effectively suppressed (as in [20]) when the incoming liquid is pre-heated to a temperature close to the saturation temperature. Regardless of the possibility of improvements in the choice of length and the inlet and exit designs of the test-section, the experiments demonstrate the ability to achieve remarkably stable pulsatile annular flows over most of the test-section. The controlling temperature-difference, reported as $\Delta \mathrm{T}$, is taken as the difference between the spatially-averaged (over $0 \leq \mathrm{x} \leq 88.5 \mathrm{~cm}$, as defined for $\Delta \mathrm{T}_{0-88.5}$ in section 2.3.1) boiling-surface temperature and the value of $\mathrm{T}_{\text {Sat|Avg }}$ for that case. The surface temperature measured by HFM-40 is reported as $\mathrm{T}_{40}$ for cases B7 - B10; however, because of a calibration issue with the sensor, for cases B1 - B6 the average of thermocouples upstream and downstream of the heat-flux meter is used for $\mathrm{T}_{40}$. The mean heat-flux in Table 2.3 is reported as $\mathrm{q}^{\prime \prime}{ }_{40}$. The measured heat-flux from HFM-40 was primarily negative because the HFM was calibrated to yield positive heat-flux values for heat absorption from the flush surface (i.e. from the fluid), as in the condensation experiments. To report positive values, the heat-flux magnitude (which represents heat flow into the fluid from the boiling surface, as in Fig. 2.3b) is reported in Table 2.3.

Flow visualization data were recorded in pictures and video. Representative videos are included in the accompanying data disc. Video 2-3 corresponds to N-IF case B1 in Table 2.3. Video 2-4 corresponds to H-IF case B2 in Table 2.3. Note that near the inlet for both cases the interfacial fluctuations resulting from the liquid flow pulsations at a frequency of $3 \mathrm{~Hz}$ is clearly visible. However, when pulsations are added to the inlet vapor flow, patches of nucleating bubbles and a rougher interface are present near the boiler inlet, their locations oscillating at $3 \mathrm{~Hz}$ in response to the vapor pressure pulsations. This is in contrast to the smoother interface near the boiler inlet observed for N-IF case B1. 


\subsubsection{Discussion of results for boiling flows in the innovative arrangement of Fig. $\mathbf{2 . 2 b}$}

For representative N-IF and H-IF cases marked as B1 and B2 in Table 2.3, Figs. 2.25 2.27 indicate a long-time, locally time-averaged, behavior of several variables. These are obtained by suitable processing of the low DAQ rate $(\sim 0.9 \mathrm{~Hz})$ time-records for these variables.

The experiments were conducted with the intent to hold the mean inlet liquid and vapor flow rates and inlet pressure approximately constant as large-amplitude fluctuations are imposed on the mean inlet pressure and flow rates. Previously discussed (section 2.2.3) liquid flow rate pulsations (amplitude and frequency) remain nearly the same for both cases.

Figure 2.25 shows that the mean inlet vapor flow rate is nearly constant as large amplitude fluctuations (in inlet vapor flow rate and pressure) are imposed on the mean inlet pressure and flow rates. Mean inlet liquid mass flow rate decreases slightly (only about $1.4 \%$ ), and inlet pressure increases slightly (only about $0.4 \%$ ) as these large fluctuation amplitudes are imposed. This is due to the constant speed of the liquid pump $P_{1}$ and use of the Evaporator controls to maintain the average inlet pressure $\bar{p}_{10}$ approximately constant as close as possible to its value for case B1.

The compressor control was used to maintain the vapor re-circulation rate as measured by coriolis meter $\mathrm{F}_{\mathrm{C}-1}$ in Fig. 2.5. The compressor speed control could have been varied to bring either the inlet liquid mass flow rate or the total inlet mass flow rate back to their values for case B1, but under those conditions the inlet vapor flow rate would probably have changed.

Under H-IF imposition of pulsations, the long time (locally time-averaged) values of $\Delta p_{0-40}$ increased by $0.6 \%$ from case B1 to case B2, but the uncertainty in the measurement of $\Delta \mathrm{p}_{0-40}$ was greater than this increase. 


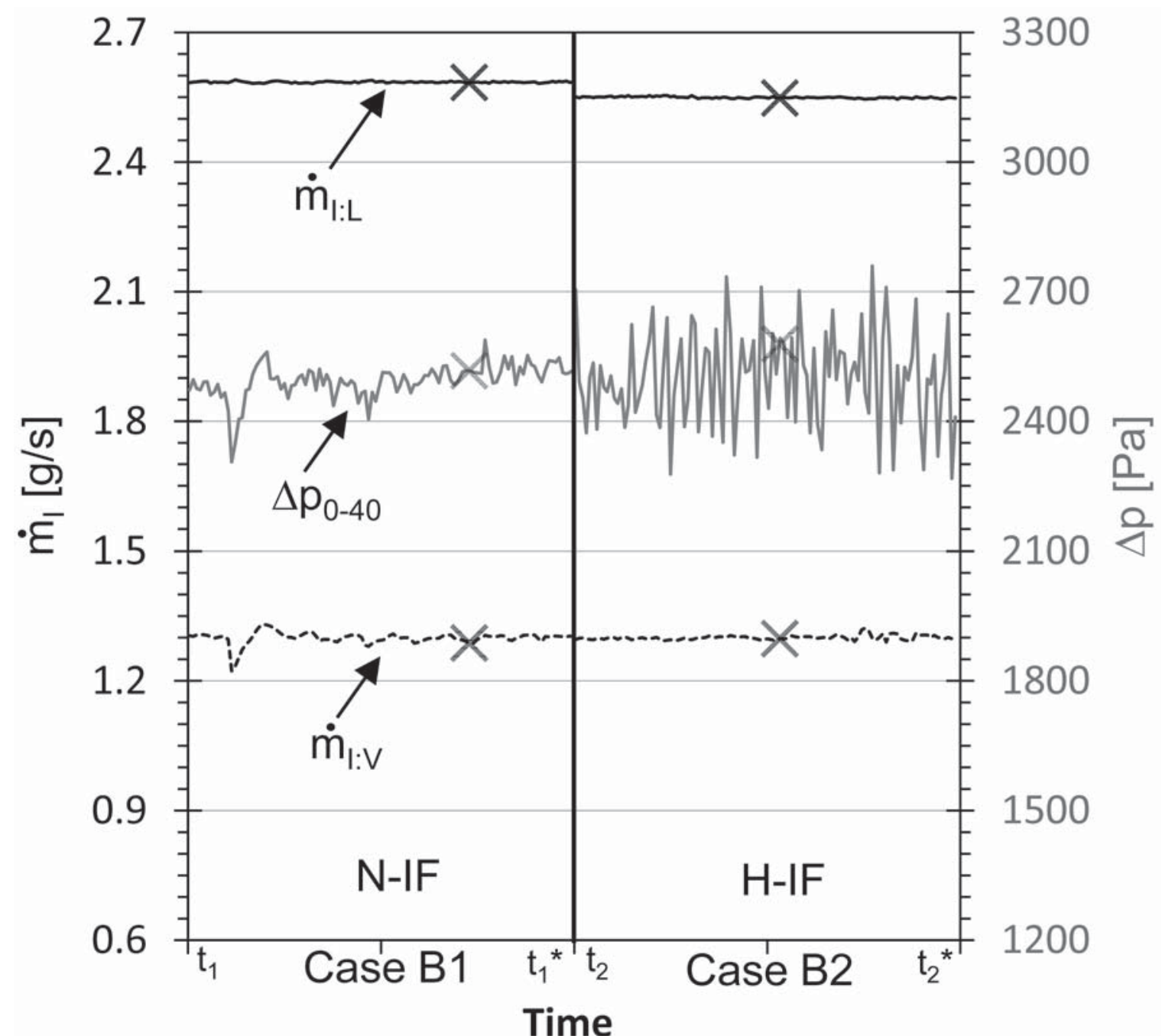

Figure 2.25. Inlet mass flow rate and pressure-difference data acquired at low DAQ rate for representative flow boiling cases. The data shown for each case are 40 minute representative subsets of the entire case data.

Figure 2.26 shows temperature $\mathrm{T}_{40}$ and heat-flux $\mathrm{q}_{40}{ }_{40}$. The values of these variables increase, to different extents, for high amplitude cases (at $\mathrm{f}_{\mathrm{P}: \mathrm{V}} \approx \mathrm{f}_{\mathrm{P}: \mathrm{L}} \approx 3 \mathrm{~Hz}$ ). Figure 2.26 and Table 2.3 show that the time-averaged heat-flux values at $\mathrm{x}=40 \mathrm{~cm}$ increase by approximately $204 \%$ in going from case B1 to case B2. 


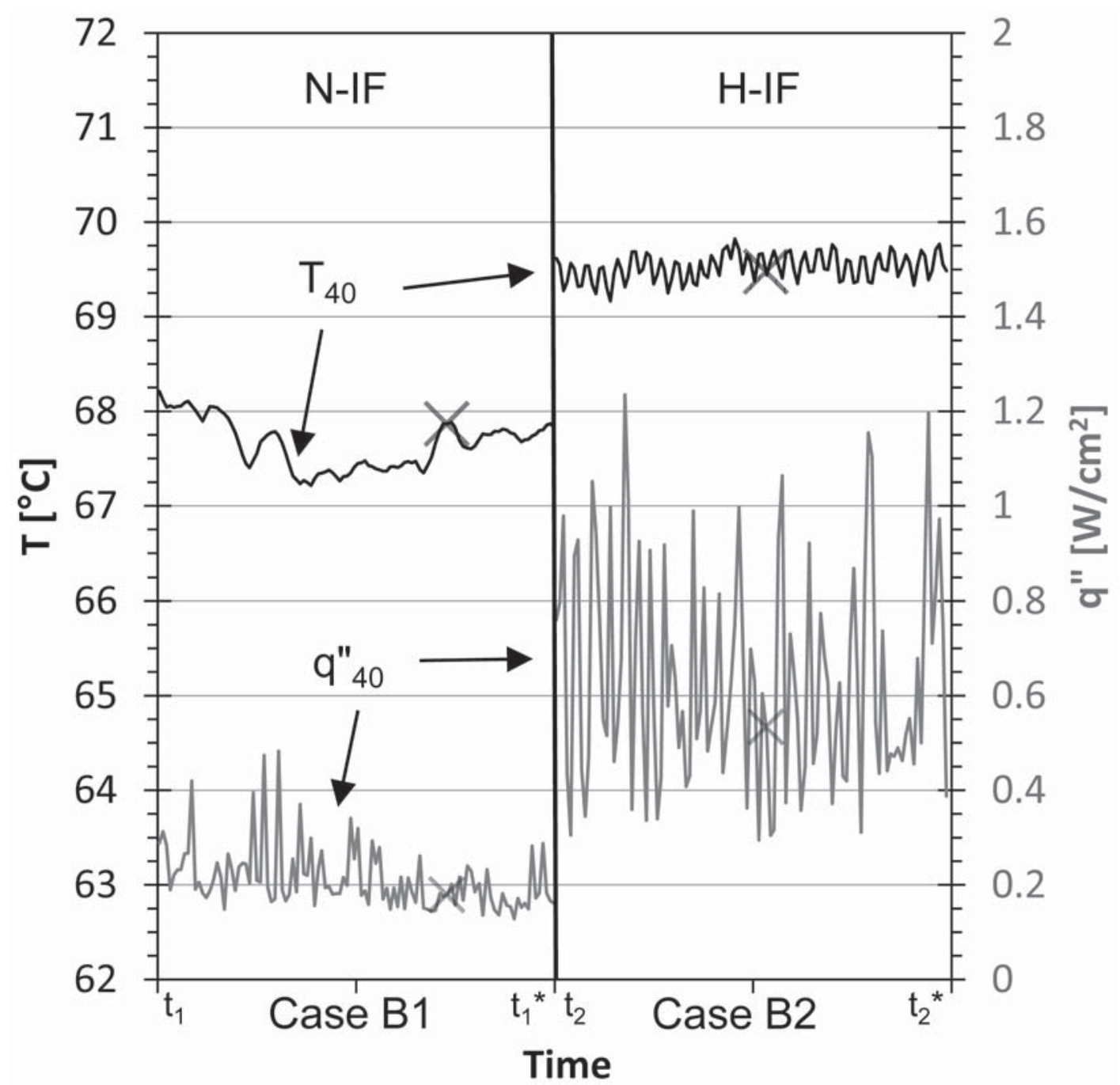

Figure 2.26. Representative local surface temperature and local heat-flux data acquired at low DAQ rate for representative flow boiling cases at the $40 \mathrm{~cm}$ location. The data shown for each case are 40 minute representative subsets of the entire case data.

Despite PID control of surface-temperatures, Fig. 2.27 shows that the spatially-varying temperatures or their mean could only be held fixed within $\pm 4{ }^{\circ} \mathrm{C}$ of the intended set points shown by dotted horizontal lines. These intended set points above the indicated saturation temperature in Fig. 2.27 were enforced by most or all of the ten TECs of Fig. 2.3c, which were operating, as described earlier, in a reversed mode. 


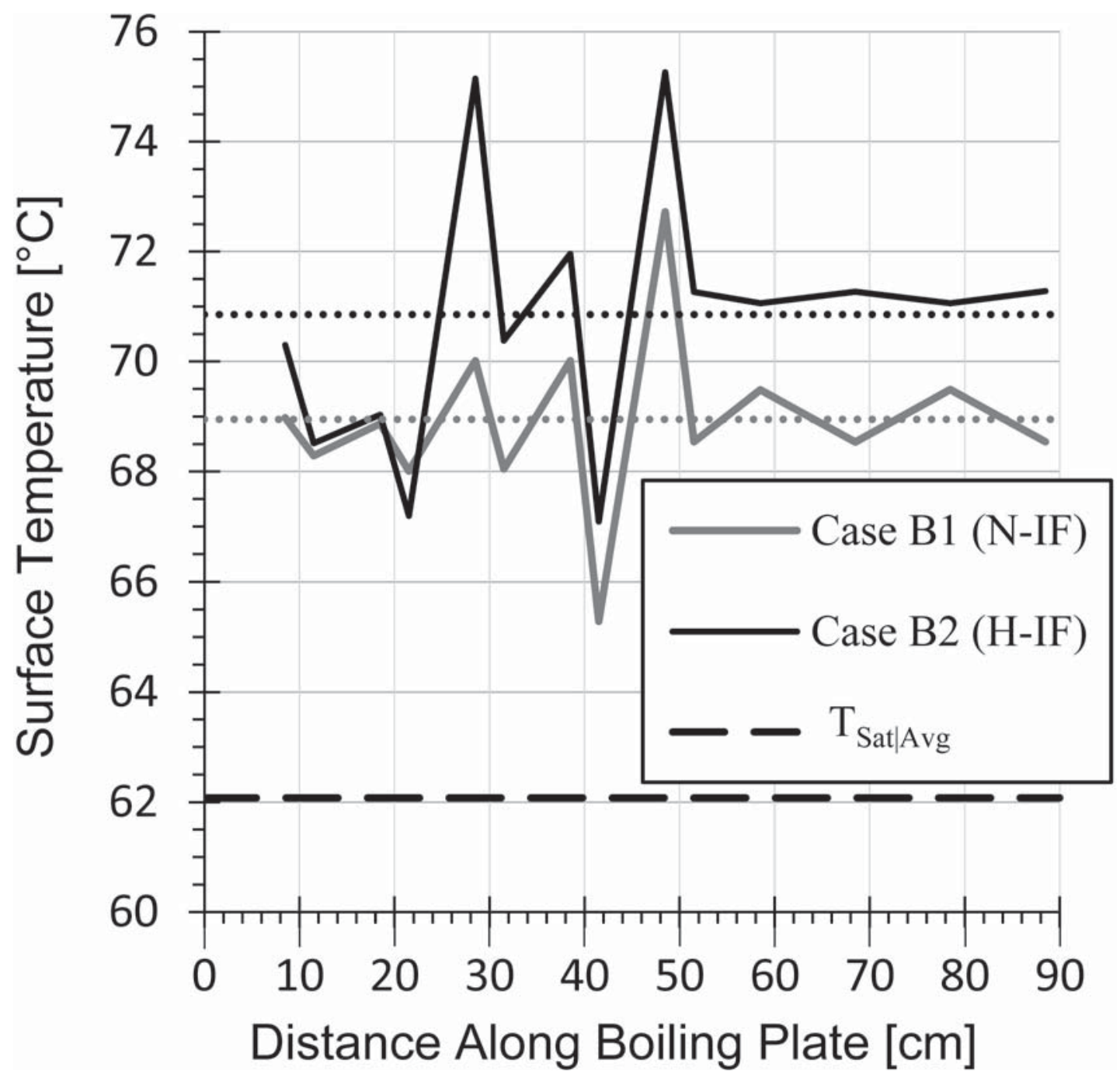

Figure 2.27. Variation of time-averaged surface temperature with distance from the test-section inlet for representative flow boiling cases. Spatially averaged surface temperatures representing the set points of the TEC controls are indicated by dotted lines.

Before going into detail, it is important to note that the first six of the ten TECs over the indicated length in Fig. 2.27 try to achieve the set point by using the mean of the two temperatures sensed through two thermocouples: one located immediately upstream and one located immediately downstream of each TEC. Because the TECs are supplying heat to the boiling fluid, these first 6 TECs tend to undershoot their set points on their upstream side and overshoot their set points on their downstream side — with the tendency being small or large. 
There are four factors which caused deviations in the wall temperatures from those specified in the TEC controls:

1. Saturation of the electrical current flowing through the thermoelectric module at its maximum value or zero - leading to long-term deviations between specific surface temperatures and their set points.

2. Local variation of temperatures within and around the region heated by each TEC — leading to the jagged shape of the temperature profiles of Fig. 2.27.

3. Time-delay between the thermal response of the heat-exchange surface and the response of the TECs - leading to sustained oscillations in time of some measured wall temperatures (not shown here for brevity).

4. The temperatures used for the feedback control of the TECs were taken from thermocouples which were improperly cold-junction compensated (corrected after the experiments) - causing all the actual wall temperatures to vary from their set points by amounts depending on the non-uniform laboratory air temperature.

The first few surface-temperatures for case B2 being significantly below their set point (approximately equal to their long-term means in Figure 2.27) is probably due to the TECs not being able to keep up with the high heat-flux caused by nucleate boiling in the film over the first $20 \mathrm{~cm}$ (of which $10-15 \mathrm{~cm}$ was visible to the naked eye in the form of bubbles on the order of a few millimeters in diameter, see Video 2-4) as the bulk liquid flow warmed to its saturation temperature.

Because the TECs used the average of their upstream and downstream surface temperatures for their feedback control, axial variations in heat-exchange surface temperature allowed the surface-temperatures to vary considerably, even when the TECs achieved their set point. For example, for case B2 the feedback-controlled temperature of the third TEC (using the average of the $4^{\text {th }}$ and $5^{\text {th }}$ data points for its temperature control) is quite close to the long-term average, while each of the individual temperatures are about $4^{\circ} \mathrm{C}$ away. The same can be seen in both case $\mathrm{B} 1$ and case $\mathrm{B} 2$ for the $5^{\text {th }}$ TEC (using the average of the $8^{\text {th }}$ and $9^{\text {th }}$ data points for its temperature control). 
The wall temperature correction (to rectify improper cold-junction compensation applied to the data during the experiments) applied to the surface-temperatures for case B1 was about $+9{ }^{\circ} \mathrm{C}$, and for case $\mathrm{B} 2$ it was about $+11^{\circ} \mathrm{C}$. This is the reason for the approximately $2{ }^{\circ} \mathrm{C}$ offset in the corrected long-term average surface-temperatures in Fig. 2.27, since the feedback controls of the TECs were relying on the uncorrected surface-temperatures.

Notice that, because the TECs are supplying heat to the boiling fluid, the downstream temperatures are almost always higher that the upstream temperatures for each TEC, and the temperatures drop in the gaps between the TECs. The flush HFM, at $x=40 \mathrm{~cm}$, is in the middle of the gap between the $4^{\text {th }}$ and $5^{\text {th }}$ TECs. After the $6^{\text {th }}$ TEC, the temperature measurements are more spread out, so the trends in surface-temperature are not as clear for $x>60 \mathrm{~cm}$.

For the N-IF and H-IF cases in Figs. 2.25 - 2.26, at points marked by the symbol "X", representative dynamic data (over a 1 second interval) obtained for $\Delta \mathrm{p}_{0-40}$ and heat-flux $\mathrm{q}_{40}$ are shown in Figs. $2.28-2.29$ below.

Figures $2.28-2.29$ again show that the causes underlying the changes in the values of $\Delta \mathrm{p}_{0-40}$ and mean values of the heat-flux $\mathrm{q}_{40}{ }_{40}$ for these annular boiling cases are also dynamic in nature and are associated with pulsations (in this case at the primary imposed frequency of $3 \mathrm{~Hz}$ ). The FFT magnitudes of these two variables, shown in Fig. 2.30, clearly show the impact of the dominant imposed frequency. 


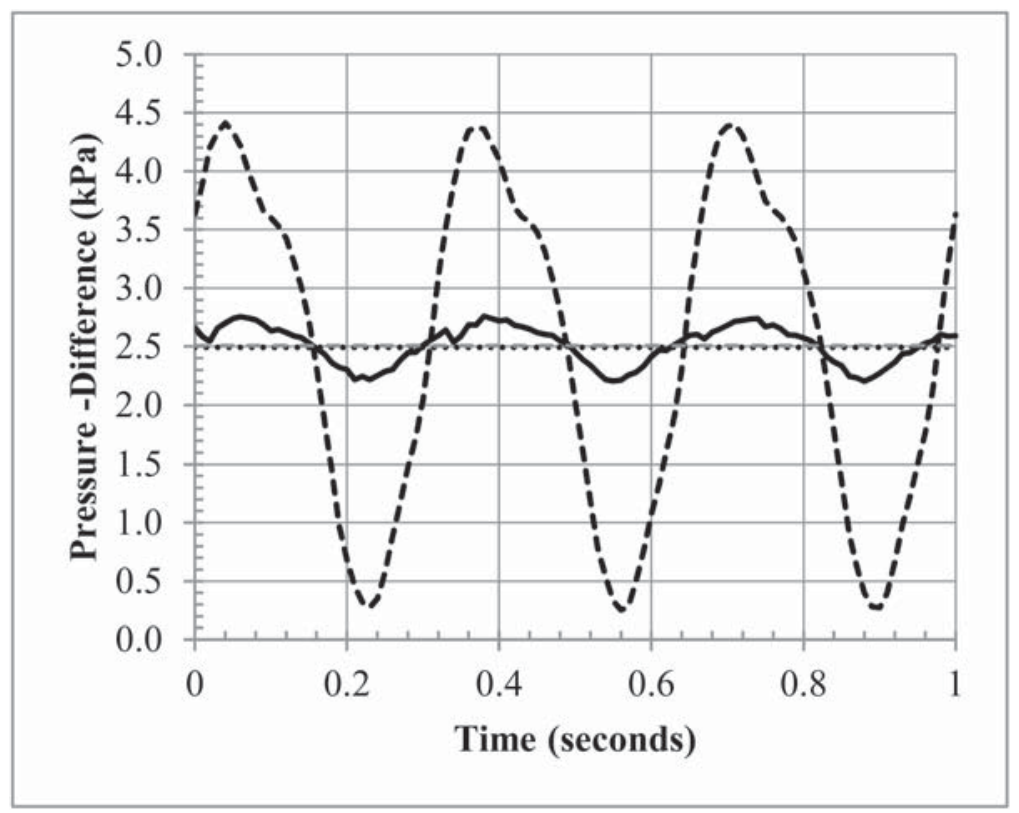

Figure 2.28. Time-varying pressure-difference $\Delta p_{0-40}$ without (solid line for case $\mathrm{B} 1$, along with dotted line indicating its average) and with (dashed line for case B2, along with light gray dashed line indicating its average) imposed inlet vapor pulsations, measured by the DPT-1 transducer between the $0 \mathrm{~cm}$ and $40 \mathrm{~cm}$ locations of Fig. 2.3b.

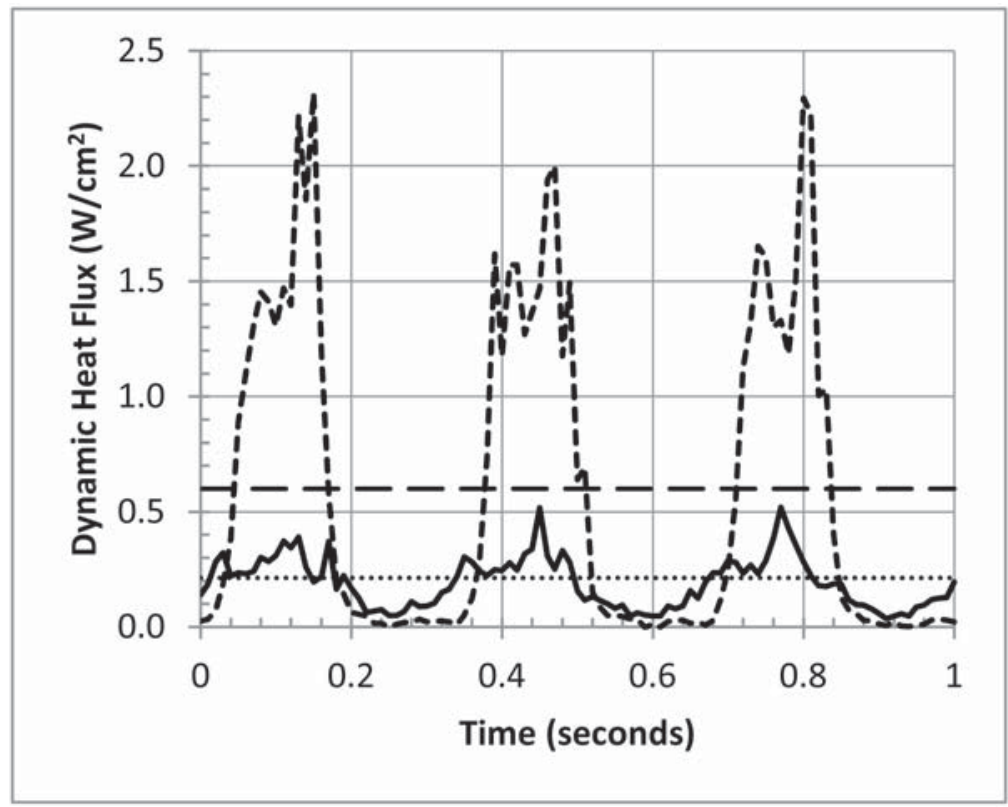

Figure 2.29. Time-varying heat-flux without (solid line for case B1, along with dotted line indicating its average) and with (dashed line for case B2, along with longer dashed line indicating its average) imposed inlet vapor pulsations, measured by a flush-type heat flux meter (HFM-40 in Figs. 2.3b-c) at the $x=40 \mathrm{~cm}$ location. 


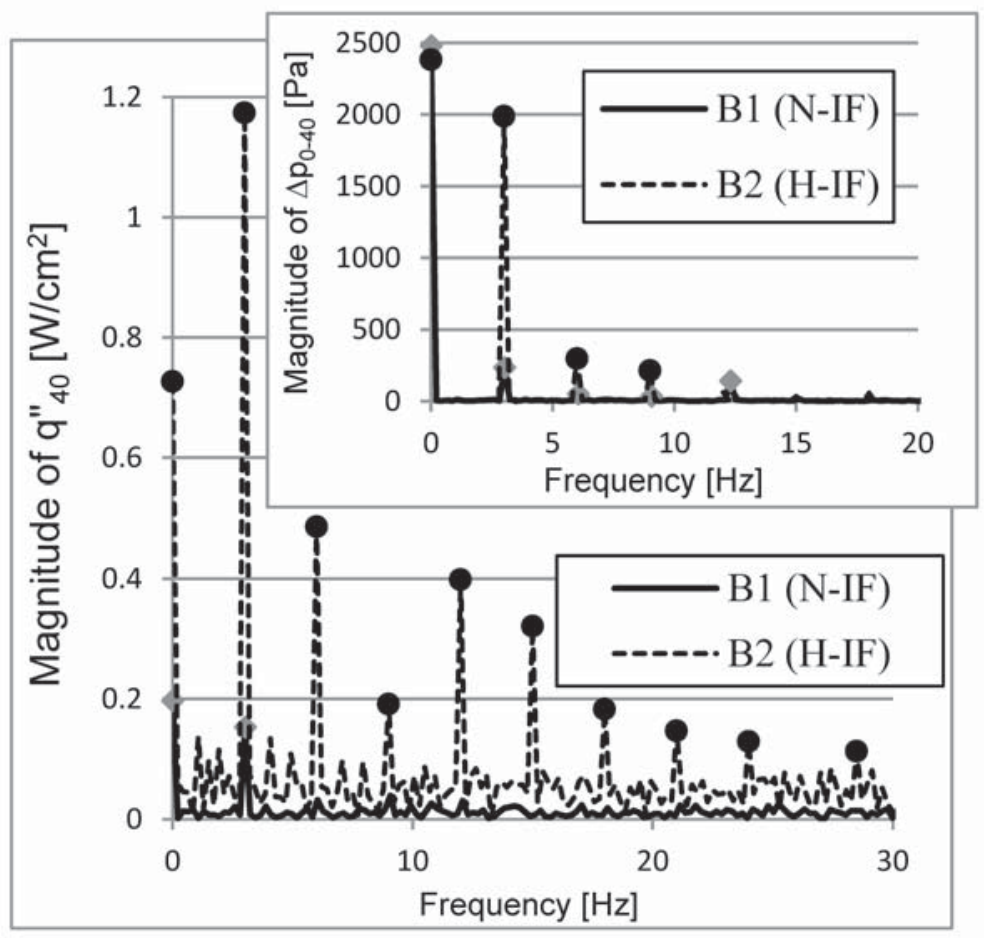

Figure 2.30. Fourier transform magnitude plots for $40 \mathrm{~cm}$ heat-flux and $0-40 \mathrm{~cm}$ pressure-difference in flow boiling. Significant peaks are marked with solid gray diamonds for case B1 and with solid black dots for case B2.

Once again the question arises as to why the interfacial waves generated by the pulsations cause the annular (as in the representative photograph in Fig. 2.2b) pulsatile film flows to change mean values of their flow variables (e.g. local heat-flux at $40 \mathrm{~cm}$ location) in a certain direction (e.g. mean local heat-flux increased by $204 \%$ in Fig. 2.29). To provide a rough understanding of the underlying reasons, and in the absence of dynamic film thickness measurements at $\mathrm{x}=40 \mathrm{~cm}$ location, we assume that when boiling film is sufficiently thin (below $200 \mu \mathrm{m}$ thicknesses) and the local film Reynolds numbers are small (say < 300), the heat flow through the dynamic liquid film is primarily by conduction. The processed dynamic film thickness values (from the knowledge that conduction heat-transfer mechanism dominates) associated with the dynamic heat-flux values in Fig. 2.29 are shown in Fig. 2.31. Again, as per discussions associated with the processing of data used to generate Figs. $2.18-2.19$ and use of molecular thermal-conductivity $\mathrm{k}_{\mathrm{L}}$ (in place of future modeling of convection effects through the 
proposed idea of an effective thermal conductivity $\mathrm{k}_{\text {eff-L }}$ ), the dynamic film thickness estimates indicated in Fig. 2.31 for the pulsatile cases at locations near wave-troughs (while thicknesses are less than $200 \mu \mathrm{m}$ ) may be smaller than their actual values.

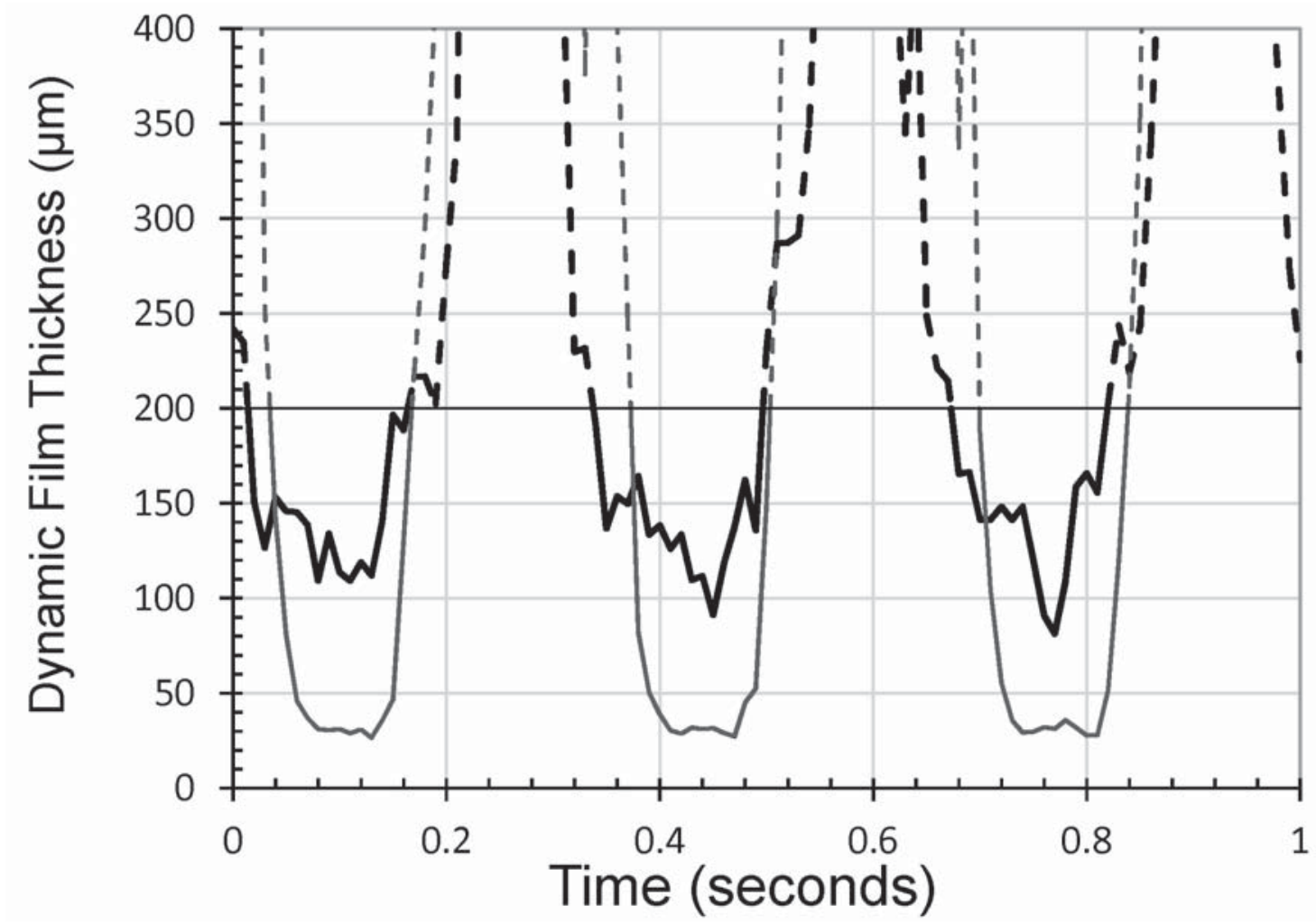

Figure 2.31. Time-varying film thickness (obtained from $\delta \equiv k \cdot\left(T_{s}-T_{\text {Sat }}\right) / q^{\prime \prime}$ under the theoretically verifiable assumption of conductive heat transfer mechanism across the film) at the $x=40 \mathrm{~cm}$ location, without (black, case B1) and with (gray, case B2) imposed inlet vapor pulsations.

The increases in time-averaged heat-flux with pulsation amplitude (as measured by the amplitude of $\mathrm{p}_{10}$ ) at various primary frequencies is again systematically observed in the experiments, and this fact is clearly seen in Fig. 2.32 for the cases in Table 2.3. 


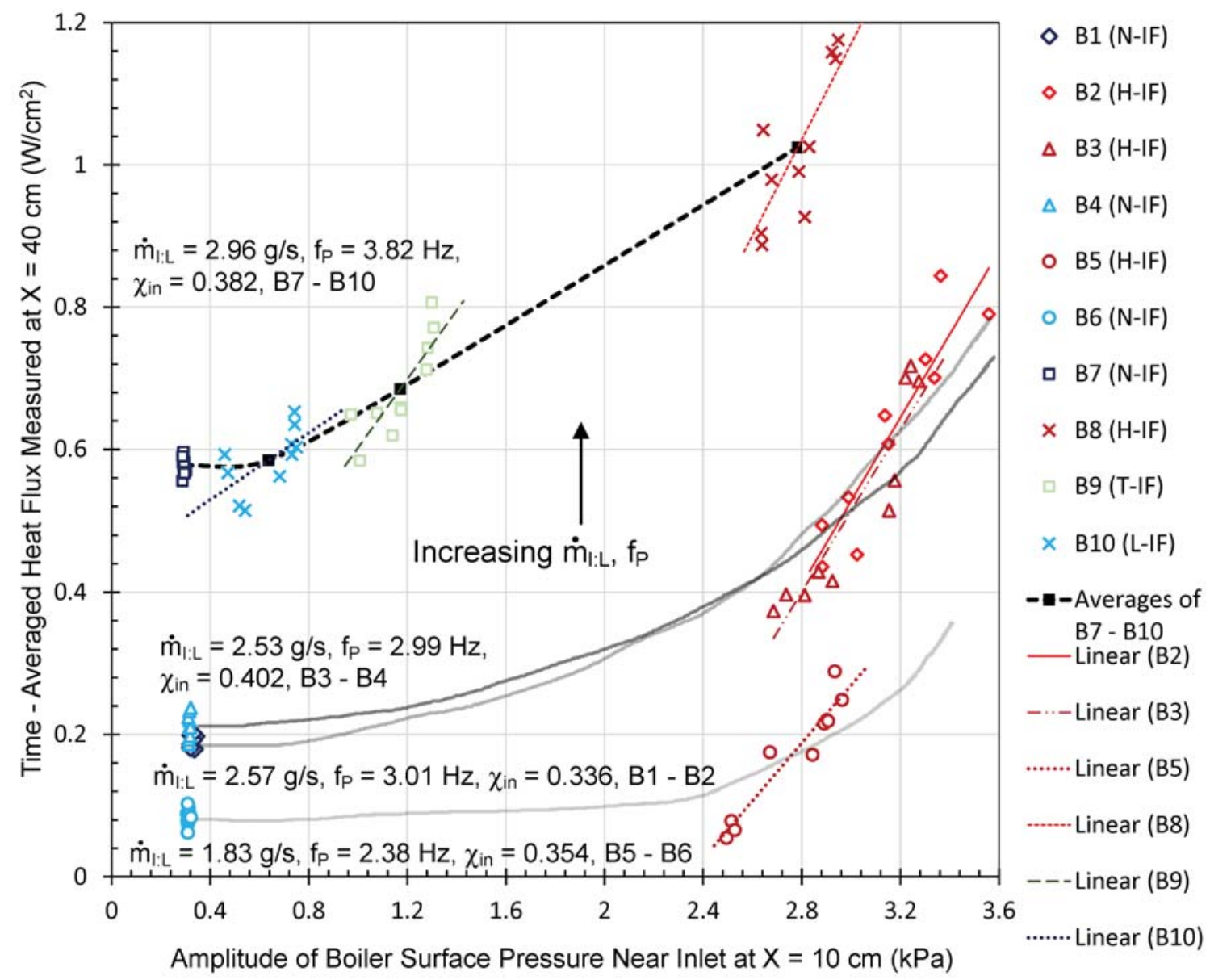

Figure 2.32. The experimentally obtained, primary dependence of time-averaged heat flux values on the amplitude of inlet pressure fluctuations $\left(a_{p / 10}\right.$ in Table 2.3) for three different frequencies (approximately 2.4, 3.0, and 3.8 Hz as indicated in Table 2.3). Alternatively, time-averaged heat flux values may be plotted with respect to amplitude of pressure-difference fluctuations $a_{\Delta p \mid 0-40}$, or local internal absolute pressure at the heat-flux measurement location $a_{p / 40^{*}}$. The groups of data points which are defined in the legend each correspond to a single time-averaged value in Table 2.3. The groups on the far left represent N-IF cases, and the groups on the right represent $\mathrm{H}$-IF cases. The straight lines fit to these groups of data may, perhaps, be interpreted in light of the fact that the absolute pressure fluctuations were measured at a different physical location $(x=10 \mathrm{~cm})$ than the time-averaged heat flux $(x=40 \mathrm{~cm})$, as discussed previously for condensing flows (see Figs. 2.21 and 2.23, and the surrounding text). 


\subsubsection{Integrated discussions}

As described earlier, the rationale for introducing inlet vapor flow rate pulsations in Fig. $2.1 \mathrm{~b}$ and Fig. $2.2 \mathrm{~b}$ (where inlet liquid flow rate pulsations are also introduced) is to generate large amplitude interfacial waves. The pulsators $\left(P_{V}\right.$ and $P_{L}$ in Fig. 2.5) allow control of the predominant amplitudes $a_{m \mid l: L}$ and $a_{m \mid l: v}$ at frequencies $f_{P: L}$ and $f_{P: v}$ that are respectively associated with the superposed pulsations on the steady-in-the-mean values of the liquid and vapor mass flow rates at the inlet of the boiler (see section 2.1 and Fig. 2.5). Based on earlier experimental experiences with pulsatile fully condensing flows (chapter 3, $[13-14,20])$ and innovative pulsatile flow boiling operations $([20,27])$, it is expected that the most energy-efficient ways of achieving the desired interfacial wavestructures throughout the length of the boiler are:

(i) using nearly resonant conditions with frequencies $\mathrm{f}_{P \cdot L} \approx \mathrm{f}_{\mathrm{P} \cdot \mathrm{V}}$ associated with the two pulsators,

(ii) ensuring incomplete boiling through the introduction of a recirculating liquid flow, and

(iii) using a passive vibrating end-plate (see Fig. 2.2b) - which is excited by the pulsatile flow - and whose principal natural fundamental frequency $f_{\text {EP } N}$ is close to the chosen value for the externally imposed frequencies $f_{P: L} \approx f_{P: V}$.

The re-circulating liquid flow approach is part of ongoing research. The vibrating end-plate features shown in the test-section of Fig. $2.2 \mathrm{~b}$ are also part of ongoing flow boiling investigations and are needed to achieve large amplitude interfacial waves throughout the length of the boiler (through interaction of forward-and-backward moving interfacial waves with each other and with the pressure/flow pulsations in the adjoining vapor phase). Presence of large amplitude interfacial waves at several locations is affected by exit condition arrangements that control reflection and/or retardation of vapor phase pressure/flow rate pulsations and interfacial waves. 


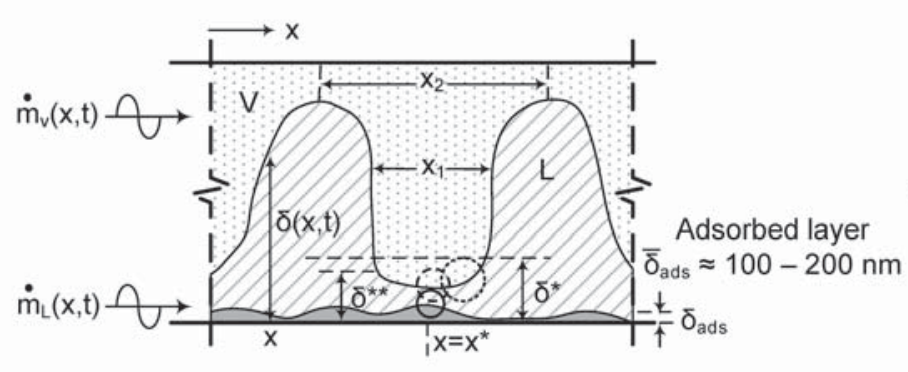

(a)

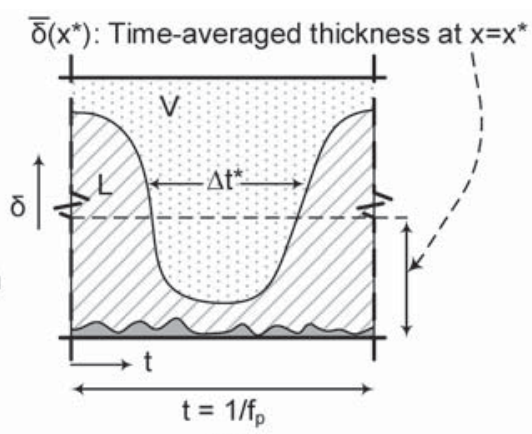

(b)

Figure 2.33. (a) Near-interface schematic of essential dynamics of the instantaneous spatial film thickness profile associated with micro-meter scale wavy film flows (over a wetting surface) encountered in pulsatile flow boiling (or flow condensation). The three lower (solid), central (dashed), and upper (dotted) intersecting circles in the figure respectively represent zones where $\mathrm{nm}$-scale, $\mu \mathrm{m}$-scale, and macro-scale phenomena interact with one another. (b) Time-varying film thickness profile at location $x=x^{\star}$ in Fig. 2.33a.

The physics underlying the significant heat-flux enhancements - for both the condensing flow results in Fig. 2.21 and the boiling flow results in Fig. 2.32 (as well as the more dramatic condensing flow enhancements reported in section 3 and [14]) - are governed by the same hypothesis that is proposed here (also see [27]) for the underlying phenomena. The hypothesis is described with the help of the schematic in Fig. 2.33, which shows large amplitude interfacial waves that can be present (and were experimentally observed, as in Videos 2-2 and 2-4) at most locations of the device. The forward or forward-and-backward moving interfacial waves that yield the instantaneous spatial profile in Fig. 2.33a are due to the pulsatile vapor and liquid flows at the inlet and are also aided by suitable exit condition arrangements. Inlet vapor flow rate pulsations provide sufficient acoustic or pulsatile energy into the vapor phase - which is in turn coupled to the rates of mechanical energy transfer across the wavy interface.

If the amplitudes are large enough relative to the local mean film thickness associated with the no-imposed pulsation case (which is in continuum scale values on the order of hundreds of $\mu \mathrm{m}$ ), the wave troughs in the schematic of Fig. 2.33a will go below a thickness $\delta^{* *}$ (on the order of tens of $\mu \mathrm{m}$ associated with micro-scale flows). The 
liquid film under these wave-troughs interacts with the liquid-vapor interface (at the intermediate dashed $\mu \mathrm{m}$-scale circle of Fig. 2.33a, where pressure - in the YoungLaplace surface tension equation - is low, and shear is low), while it concurrently interacts with the adsorbed layer (the lower solid nm-scale circle in Fig. 2.33a).

The actual thickness of this adsorbed layer is not important to the analysis here; what is important is that it forms a boundary condition of the very thin micro-layer, which must also respond to the liquid-vapor interface and the macro-scale eddy currents immediately upstream and downstream of the wave trough. The thinness of the micro-layer under the liquid-vapor interfacial wave troughs (order $10-100 \mu \mathrm{m}$ ) dictates that the physical response of this molecular thickness adsorbed layer be taken into account when modeling the boundary condition between the liquid and the heat exchange surface.

The wave-troughs may interact with and change (or destabilize) the very nature of the nano-meter scale, solid-like adsorbed layer (indicated by the dark grey shaded zone of characteristic thickness $\delta_{\text {ads }}$ - in Fig. 2.33a), which is capable of changing the sign of disjoining pressures (see p. 89 and p. 371 of [4], [28 - 30]) and reacts to shear-stress variations in a manner that is different from the fluid-like continuum response. This special solid-like behavior of the adsorbed layer arises from Van der Waal forces between the fluid and the wetting surface [31]. Note that at any representative location (like that shown in Fig. 2.33a), for the time durations over which $\delta(x, t)<\delta^{* *}$, disjoining pressure effects in the adsorbed layer over the wetting solid surface can change its nature (e.g. can make its normal-stress-state negative or tensile) relative to its neighboring (underneath the continuum layer) values towards accommodating the more complex velocity profiles needed in the micro-layer (see the lower and central intersecting circles in Fig. 2.33a).

As indicated by the central and upper intersecting circles in Fig. 2.33a, the micro-layer in turn responds to the demands made by the macro-layer. More complex time-varying axial and transverse velocity and temperature profiles at wave-trough locations are needed to meet the matching requirements at the interface (mass-flux, surface-tension coupled 
pressures, and temperature restrictions) and in the thicker continuum-layer that may be present in the adjacent portions experiencing wave-crests.

It is hypothesized that the above-described flow physics causes wave-troughs to "stick" or "dwell" near the surface while allowing for the low pressures and shear to allow the lateral liquid flow along the heat-exchange surface to effectively negotiate the constricted wave-trough thickness. This means that the time duration $\Delta t^{*}$ (see Fig. 2.33b) associated with the length $\mathrm{x}_{1}$ (in Fig. 2.33a) of the "sticking" wave-trough at $\mathrm{x}^{*}$ is a very significant fraction of the externally imposed pulsation's time-scale of $1 / f_{p}$ - which is associated with transit distance $\mathrm{x}_{2}$ around location $\mathrm{x}^{*}$.

This hypothesis is supported by the result in Figs. 2.21 and 2.32 and numerous related experimental results for thin film pulsatile condensing flows (chapter 3, [13-14, 27]). Because of this, the long-time averages (over several hundred pulsation time-scales $1 / f_{p}$ ) of film thickness values could be significantly smaller for these pulsatile flows than for the corresponding non-pulsatile cases (not shown in Fig. 2.33). As a result, as shown in Fig. 2.29, heat-flux (which is assumed to be inversely proportional to instantaneous film thickness - whose values may be larger than the lower threshold values shown in Fig. 2.31) significantly increases for pulsatile flow innovative boilers (as also with innovative condensers here (see Figs. 2.18 - 2.19) and in [27, $32-33]$ ).

Though rigorous understanding of the above-described mechanisms is not available at this time, it is conjectured that at least part of this mechanism (at the lower solid circle in Fig. 2.33a) is a variation of the well-documented nucleate boiling mechanism ([30]) where micro-layer liquid films interact with nm-scale adsorbed layers leading to a very significant heat-flux enhancement at the contact line of a growing and detaching vapor bubble (surrounded by its liquid-phase) from a heated, wetting surface.

The novelty of the proposed approach lies in making phenomena at three different length scales - continuum, micro-scale, and nano-scale - to interact with phenomena at two 
different time-scales $(0.05-0.2 \mathrm{~s}$ associated with $5-20 \mathrm{~Hz}$ imposed frequencies $\mathrm{f}_{\mathrm{L}} \approx \mathrm{f}_{\mathrm{V}}$ and the longer time scales of minutes associated with the time-averaged mean mass flow rates, film thicknesses, etc.) in a way that significantly and beneficially changes the lon-term mean values of the flow variables over the entire length of the phase-change device.

Since these changes are affected by the dynamic time-varying structure of interfacial waves, the mean flow behavior is affected by the presence or absence of standing interfacial waves (which locally move forward and backward) of sufficient amplitude. These factors depend on the nature of physical arrangements at the exit of the boiler/condenser. Therefore, though the vapor and liquid flows are primarily incompressible, the mean values of the flow fields affected by the superposed wavestructure exhibit "elliptic sensitivity" (i.e. exit condition dependency).

Associated with these phenomena, in going from non-pulsatile to pulsatile cases, there is a change in resistance to the vapor flow (and associated forward-and-downward push on the liquid flow). This is analogous to changing the interface from a smooth-soled shoe to one with a good tread (wave-induced texturing) which also develops, as necessary, suitable longer spikes (e.g. suction due to disjoining pressure effects) on the tread.

The introduction of the right amount of energy-efficient liquid and vapor phase recirculation rates - with superposed pulsations at a dominant amplitude and frequency - also desensitizes the otherwise sensitive annular flows to random and ever-present time-variations in absolute pressure that the boiler or condenser will otherwise see in any practical application. This was observed in the full dynamic time histories of $\mathrm{p}_{10}$, in which the N-IF cases showed significant excursions in $\mathrm{p}_{10}$ values over 3 seconds and longer time periods; the averaged and shorter time histories reported in Figs. $2.14-2.15$ for condensing flows do not show this phenomenon. These pressure variations were reduced for corresponding $\mathrm{H}-\mathrm{IF}$ cases. 
From the time-domain response of the film thickness estimates in Figs. $2.18-2.19$, it is clear that case C10 (see Fig. 2.19), which exhibited the highest heat-flux enhancement, was primarily influenced by the "sticking" phenomenon caused by solid-liquid-vapor interaction as described above. However, for case C2 (see Fig. 2.18), the shape of the film thickness profile suggests the significant influence of a physical phenomenon limiting the height of the wave peaks at thicknesses too large for the near-surface phenomenon discussed with regard to Fig. 2.33. The "sticking" phenomenon may still be occurring at the wave-troughs, which get down to approximately $40 \mu \mathrm{m}$ film thickness. However, the flattening of the interfacial wave peaks in time occurs at calculated liquid film thicknesses around $150 \mu \mathrm{m}$, so it is likely the result of a different physical phenomenon.

The answer may be found in the above-mentioned "interface texturing" effect, which involves the dynamics of the instantaneous velocity fields in the liquid and vapor phases near peaks in the interfacial waves. Details of this "interface texturing" phenomenon are discussed in chapter 3 and shown in slow motion in Video 3-3. Note the small film thickness fluctuations at the peaks of the film thickness profile for case C2 in Fig. 2.18, which may be indicative of "secondary waves" (see section 3.4.5) passing over the heat-flux meter.

\subsection{Conclusions}

The main conclusions are:

- The proposed innovative condenser (Fig. 2.1b) and boiler (Fig. 2.2b) operations can successfully use re-circulating vapor flows to ensure realization of thermally and hydrodynamically efficient annular flows over most of the devices' heat-exchange surfaces. These surfaces are therefore continuously irrigated by thin liquid films.

- $\quad$ By superposing relatively fast time-scale $(2.5-30 \mathrm{~Hz})$ flow rate pulsations of sufficient amplitude on annular boiling/condensing flow realizations, large amplitude interfacial waves can be generated, which influence the flow variables at much slower time scales $(<0.01 \mathrm{~Hz})$. This is due to significant reductions in instantaneous film thickness values over a significant portion of a typical time-period associated with 
externally imposed flow rate oscillations. As a result there are dramatic reductions in the mean film thickness values associated with long time (> $100 \mathrm{~s}$ ) averages and, also, significant enhancements in time-averaged heat-flux values (100 - 1000 \%).

- The above approach continues to keep annular regimes stable and, because of the dominance of imposed pulsations, the otherwise sensitive shear-driven flow realizations are desensitized to inadvertent, system-induced time-variations in absolute pressure.

- $\quad$ By suitable future design changes (with regard to choices of: device length for a specified range of inlet liquid/vapor flow rates that allow non-zero liquid flow rates at the exit of the innovative boiler, exit geometries that reflect interfacial waves and aid in enhancing interfacial wave amplitudes, inlet geometry and temperature conditions for controlling entrainment and nucleation phenomena, large latent heat working fluids such as water, etc.), it is expected that the reported local heat-flux enhancements will occur over most of the test-section towards realization of high heat-flux $\left(>1 \mathrm{~kW} / \mathrm{cm}^{2}\right)$ and functional $\mathrm{mm}$-scale shear-driven flow boilers.

Acknowledgment: This work was supported by NSF Grant CBET-1033591. 


\section{References: chapter 2}

[1] M.T. Kivisalu, P. Gorgitrattanagul, A. Narain, Results for high heat-flux flow realizations in innovative operations of milli-meter scale condensers and boilers, International Journal of Heat and Mass Transfer, 75 (2014) 381-398.

[2] S.G. Kandlikar, H. Narai, M. Shoji, V.K. Dhir, Handbook of Phase Change: Boiling and Condensation, Taylor and Francis, New York, 1999.

[3] S. Nukiyama, The maximum and minimum values of the heat $Q$ transmitted from metal to boiling water under atmospheric pressure, International Journal of Heat and Mass Transfer, 9(12) (1966) 1419-1433.

[4] V.P. Carey, Liquid-Vapor Phase-Change Phenomena, Series in Chemical and Mechanical Engineering, Taylor and Francis Group, $2^{\text {nd }}$ ed., 2008.

[5] J.H.I. Lienhard, J.H.V. Lienhard, A Heat Transfer Textbook, Phlogiston Press, Cambridge, MA, 2003.

[6] J.H. Lienhard, V.K. Dhir, D.M. Riherd, Peak Pool Boiling Heat-Flux Measurements on Finite Horizontal Flat Plates, Journal of Heat Transfer, 95(4) (1973) 477-482.

[7] C.J. Kuo, Y. Peles, Flow Boiling of Coolant (HFE-7000) Inside Structured and Plain Wall Microchannels, Journal of Heat Transfer, 131(12) (2009) 121011-121019.

[8] T. Harirchian, S.V. Garimella, Effects of channel dimension, heat flux, and mass flux on flow boiling regimes in microchannels, International Journal of Multiphase Flow, 35(4) (2009) 349-362.

[9] W. Qu, I. Mudawar, Measurement and correlation of critical heat flux in two-phase microchannel heat sinks, International Journal of Heat and Mass Transfer, 47(10-11) (2004) 2045-2059.

[10] A.E. Bergles, S.G. Kandlikar, On the Nature of Critical Heat Flux in Microchannels, Journal of Heat Transfer, 127(1) (2005) 101-107.

[11] S.R. Choi, J.W. Evangelista, C.T. Avedisian, W. Tsang, Experimental study of chemical conversion of methanol and ethylene glycol in a film boiling reactor, International Journal of Heat and Mass Transfer, 54(1-3) (2011) 500-511.

[12] M. Li, C. Dang, X. Fu, Y. Ma, Predictions for Saturated Flow Boiling Heat Transfer in MiniChannels, in: Proceedings of the ASME 2012 Summer Heat Transfer Conference, (2012). [13] M. Kivisalu, P. Gorgitrattanagul, S. Mitra, R. Naik, A. Narain, Prediction and Control of Internal Condensing Flows in the Experimental Context of their Inlet Condition Sensitivities, Microgravity Sci. Technol., 24(3) (2012) 147-155. 
[14] M.T. Kivisalu, P. Gorgitrattanagul, A. Narain, R. Naik, M. Hasan, Sensitivity of shear-driven internal condensing flows to pressure fluctuations and its utilization for heat flux enhancements, International Journal of Heat and Mass Transfer, 56(1-2) (2013) 758-774.

[15] J.H. Goodykoontz, R.G. Dorsch, Local heat-transfer coefficients for condensation of steam in vertical downflow within a 5/8-inch-diameter tube, NASA TN D-3326 (1966).

[16] A. Cavallini, R. Zecchin, High Velocity Condensation of R-11 Vapors Inside Vertical Tubes, in: Studies on Heat Transfer in Refrigeration, Proc. International Institute of Refrigeration, Trondheim, Norway, (1971) 385-396.

[17] M.M. Shah, A general correlation for heat transfer during film condensation inside pipes, International Journal of Heat and Mass Transfer, 22(4) (1979) 547-556.

[18] A. Cavallini, J.R. Smith, R. Zechchin, A Dimensionless Correlation for Heat Transfer in Forced Convection Condensation, in: $6^{\text {th }}$ International Heat Transfer Conference, Tokyo, Japan, (1974).

[19] S. Mitra, A. Narain, R. Naik, S.D. Kulkarni, A quasi one-dimensional method and results for steady annular/stratified shear and gravity driven condensing flows, International Journal of Heat and Mass Transfer, 54(15-16) (2011) 3761-3776.

[20] A. Narain, M. Kivisalu, R. Naik, N. Gorgitrattanagul, S. Mitra, M.M. Hasan, Comparative Experimental and Computational Studies for Annular Condensing and Boiling Flows in Milli-meter Scale Horizontal Ducts. Paper No. HT2012-58602, pp. 563-574; doi:10.1115/HT2012-58602, Proceedings of ASME 2012 Summer Heat Transfer Conference. Rio Grande, Puerto Rico, USA (2012).

[21] S. Mitra, Development of one-dimensional and two-dimensional computational tools that simulate steady annular internal condensing flows in terrestrial and zero-gravity environments, Ph.D. Dissertation, Michigan Technological University, Houghton, MI, 2012.

[22] R. Naik, Development of unsteady two-dimensional computational simulation tools that simulate steady annular internal condensing flows and characterize interfacial waves, flow stability and flow sensitivity, Ph.D. Dissertation, Michigan Technological University, Houghton, MI, 2014.

[23] A. Narain, Q. Liang, G. Yu, X. Wang, Direct Computational Simulations for Internal Condensing Flows and Results on Attainability/Stability of Steady Solutions, Their Intrinsic Waviness, and Their Noise Sensitivity, Journal of Applied Mechanics, 71(1) (2004) 69-88. [24] Naik R., Mitra S., Narain A., Shankar N., Steady and Unsteady Computational Results of Full Two Dimensional Governing Equations for Annular Internal Condensing Flows, Computational Fluid Dynamics (http://www.comsol.com/conference2013/usa/presentations/), COMSOL Conference, October 9 - 11, 2013, Boston (2013). 
[25] A. Narain, N. Ajotikar, M.T. Kivisalu, A. Rice, M. Zhao, N. Shankar, Obtaining Time-Varying Pulsatile Gas Flow Rates With the Help of Dynamic Pressure-Difference and Other Measurements for an Orifice-Plate Meter, Journal of Fluids Engineering, 135(041101) (2013) $1-19$.

[26] W. Kays, M. Crawford, B. Weigand, Convective Heat and Mass Transfer, McGraw-Hill, $3^{\text {rd }}$ edition, 1987.

[27] A. Narain, M.T. Kivisalu, P. Gorgitrattanagul, R. Naik, N. Shankar, Results for High Heat-flux Realizations in Innovative Operations of Milli-meter Scale Condensers and Boilers. Paper number: HMTC 1300183 , Proceedings of: $22^{\text {nd }}$ National and $11^{\text {th }}$ International ISHMTASME Heat and Mass Transfer Conference, IIT Kharagpur, India, December 28 - 31, (2013). [28] C. Kunkelmann, K. Ibrahem, N. Schweizer, S. Herbert, P. Stephan, T. Gambaryan-Roisman, The effect of three-phase contact line speed on local evaporative heat transfer: Experimental and numerical investigations, International Journal of Heat and Mass Transfer, 55(7-8) (2012) 1896-1904.

[29] P.C. Stephan, C.A. Busse, Analysis of the heat transfer coefficient of grooved heat pipe evaporator walls, International Journal of Heat and Mass Transfer, 35(2) (1992) 383-391.

[30] J.G. Truong, J.P.C. Wayner, Effects of capillary and van der Waals dispersion forces on the equilibrium profile of a wetting liquid: Theory and experiment, The Journal of Chemical Physics, 87(7) (1987) 4180-4188.

[31] B.V. Derjaguin, V.M. Muller, Y.P. Toporov, Effect of contact deformations on the adhesion of particles, Journal of Colloid and Interface Science, 53(2) (1975) 314-326.

[32] NSF CBET-1033591 research accomplishments - High Effectiveness Microscale Condensers and Boilers for Terrestrial and Space Applications, FY 2012 (http://www.nsf.gov/eng/cbet/achieve/1406/2012/narain.htm).

[33] Research.gov NSF Research Award Highlights - Awarded 2010, Adapting Boilers and Condensers for Earth and Space: Flow Prediction and Fluctuation Sensitivity Investigations for Quasi-Steady Shear Driven Condensing Flows in Milli-meter to Micro-meter Scale Two Phase Systems (2013) (https://www.research.gov, search for "Narain"). 


\section{Completely condensing flow experiments ${ }^{2}$}

The text of this chapter has been edited from its originally published version [1]. New figures have been added, and some existing figures have been edited for content and/or re-formatted to fit the structure of this dissertation. These changes reflect information that was not available at the time of the original publication and material that was available but was left out of the originally published version due to space constraints.

\section{Abstract: chapter 3}

The reported experimental results are for annular zones of fully condensing flows of pure FC-72 (perfluorohexane) vapor. The flow condenses on the bottom surface (316 stainless steel) of a horizontal, rectangular cross-section duct. The sides and top of the duct are made of clear plastic. The annular portion of the flow in the test-section is driven, under negligible to zero gravity effects along the flow direction, by pressure-difference and cooling conditions. Since the annular regime condensate motion is primarily driven by an effective interfacial shear stress, all such flows are termed shear-driven flows. The experimental system in which this condenser is used is able to control steady-in-the-mean (termed quasi-steady) values of inlet mass flow rate, inlet (or exit) pressure, and wall cooling conditions. For the experimental results reported here, the mean (time-averaged) inlet mass flow rate, mean inlet pressure, and condensing-surface cooling conditions were held fixed at their quasi-steady values.

Under these conditions, it was found that the imposition of volume flow rate pulsations upstream of the condenser inlet led to small condenser inlet pressure fluctuations (relative to the mean inlet pressure). However, these inlet pressure fluctuations were large when

\footnotetext{
2 Large parts of this chapter are taken from: M.T. Kivisalu, P. Gorgitrattanagul, A. Narain, R. Naik, M. Hasan, Sensitivity of shear-driven internal condensing flows to pressure fluctuations and its utilization for heat flux enhancements, International Journal of Heat and Mass Transfer, 56 (2013) 758-774. It is listed as [1] in the list of references. However, parts of the version reported here have been modified/improved since their original publication as described in the preface and summarized in the first paragraph of this chapter. These and any other changes are not endorsed by the publisher. Permission to reproduce [1] has been granted by the publisher, Elsevier Limited. The license agreement is available upon request from the author, who may be contacted via email at mtkivisa@mtu.edu.
} 
compared with the mean pressure-difference across the condenser, so they induced large pressure-difference fluctuations across the condenser, commensurate with significant mass flow rate pulsations within the condenser. When these pulsations are present, there is a change in the very nature of the quasi-steady annular condensing flow regime.

The resulting phenomena change the mean local heat-flux values with significant (> $200 \%$ ) enhancements. There are accompanying time-varying changes in the liquid-vapor configurations within the annular and the non-annular flow regimes. This changes the mean and fluctuation amplitude values (with induced harmonics) in the absolute pressure at any interior location within the annular flow regime. It is shown here that the heat-flux enhancement phenomenon is real and occurs regardless of the method of cooling for a suitable range of fluctuation frequencies and amplitudes.

This chapter experimentally investigates how the strength of this sensitivity varies with amplitude and frequency of pressure or mass flow rate fluctuations imposed at the inlet of the condenser. Associated theory and rudimentary experiments (not reported here, see chapter 2) suggest that similar enhancement may be observed in annular flows which do not completely condense before the exit, provided that suitable arrangements at the condenser exit allow similar or equivalent liquid-vapor interfacial wave structures with the help of similar acoustic wave reflections in the vapor phase.

Keywords: horizontal channel condensation, fluctuation sensitivity, internal condensing flows, flow pulsations, annular flows, heat flux enhancements

\subsection{I ntroduction and nomenclature}

This chapter (see also journal article [1]) presents an experimental investigation (with flow visualization) of the sensitivity of shear-driven internal condensing flows to pulsations in the inlet vapor flow rate and/or inlet pressure. Furthermore, the focus is primarily on the quasi-steady annular regime of these flows. Fully condensing flows with condensation on the bottom plate of a horizontal channel are considered. The 
quasi-steady flows with small pulsation amplitudes are also termed "nearly steady," whereas large amplitude cases are also termed "pulsatile."

These shear-driven internal condensing flows are of interest here because they occur in horizontal, micro-gravity, and micro-meter scale hydraulic diameter ducts. Such ducts have applications in next generation space-based and military aircraft thermal management systems, as well as in high power electronic cooling applications [2 - 3].

Although the mm-scale results reported here may also apply to micro-meter scale ducts, the influences of additional surface tension and transient compressibility (due to higher pressure drops) effects in micro-scale ducts are higher due to their small cross-sectional and large surface areas - perhaps contributing to frequently observed non-annular and transient condensing flows [4 -6]. Although drop-wise condensation [7] and associated surface-enhancement techniques are being developed for compact, non-traditional thermal management systems [8], this chapter does not deal with those situations or applications.

For condensing flows in mm-scale or larger hydraulic diameter ducts, there are many papers that deal with measurements of heat-transfer coefficients associated with condensation of pure vapors flowing inside vertical or horizontal ducts (of circular or rectangular cross-sections, as in [9-12]). The experiments, as well as related correlations in the literature [13 - 22], typically cover a very large set of flow regimes and associated flow physics categories (as defined in [23 - 24]). Although pulsatile single-phase flow investigations and experimental observations with regard to heat-transfer enhancements have been reported [25 - 27], analogous investigations for phase-change condensing flows have been limited [28] and have not included amplitude-frequency characterizations of the flows' response. Because of the importance of such investigations to the design of new thermal systems [29], this chapter, and its corresponding paper [1], contribute to quantitative, qualitative, and visual experimental characterization of the annular regime's heat-transfer enhancement as it relates to the amplitude-frequency 
response of shear-driven internal condensing flows.

In this chapter (and corresponding journal paper [1]), the sensitivity of these flows to the amplitude and frequency of imposed inlet pressure fluctuations (and associated inlet flow rate pulsations) are investigated.

Such inadvertently or deliberately imposed fluctuations commonly occur in closed flow loops in which the condensing flow is primarily shear-driven, and devices like turbines or reciprocating compressors introduce significant pressure pulsations to the vapor supplied to the condenser. Imposed pressure fluctuation cases reported here re-establish preliminary results reported earlier in $[30-31]$ by considering a larger data matrix that involves a range of amplitudes and frequencies under two different cooling conditions. Besides, part of the goal is to unambiguously establish the fact that, in comparison to low amplitude pulsations, high amplitude pulsations significantly change (sometimes $>200 \%$ ) the time-averaged (mean) heat-flux values associated with the annular regime of the resulting quasi-steady condensing flow realizations.

Pressure pulsation amplitudes on the order of 100 - $1500 \mathrm{~Pa}$ (for a mean inlet absolute pressure of $142 \mathrm{kPa}$, representative of a $50-200 \mathrm{kPa}$ range of interest) induce large pulsations in the inlet mass flow rate. Since the mean pressure-differences across the annular lengths of mm-scale (or larger) hydraulic diameter condensers are often only on the order of $100-1000 \mathrm{~Pa}$, such pressure fluctuation amplitudes imposed at the condenser inlet significantly affect the annular flow condensation.

These shear-driven flows, with zero to negligible gravitational assistance, are much more sensitive to pressure/flow fluctuations than gravity-driven condensing flows. Thus, they need very different specification and control of inlet/exit conditions in the presence of commonly induced pressure fluctuations/flow pulsations to ensure meaningful and repeatable experimental data. In contrast, gravity-driven condensate motion leads to long and robust annular flow regimes that do not show any significant sensitivity to levels of inlet pressure or flow rate fluctuations similar to the ones imposed here on shear-driven 
condensing flows [24].

Another objective of this paper is to report and advance prediction and control capabilities for shear-driven internal condensing flows in the context of their unique sensitivities to inadvertent or deliberate impositions of fluctuations/pulsations on the mean values of inlet pressure and/or flow rate. This is to be accomplished by experimentally and theoretically assessing the knowledge (or control) of boundary conditions necessary to ensure repeatability of pulsatile shear-driven flows. Such assessments also benefit from a systematic analysis of the sensitivity mechanisms underlying the physical causes of the annular regime heat-flux enhancement.

The fluctuation sensitivity results reported here with regard to the length of the annular regime (and loss of annularity), if understood with the help of suitable computational simulation capabilities, will also contribute to the development of an understanding of the variety of complex flow morphologies [4 - 5] and transient phenomena [32 - 34] that are possible for shear-driven condensing flows. Results similar to those reported here are expected to hold for annular flow boiling (with an innovative combination of liquid and vapor flows at the boiler inlet [35]), and are currently being experimentally established (see chapter 2 for preliminary flow boiling experimental results).

\section{Nomenclature}

$\mathbf{a}_{\mathrm{r} \mid \mathrm{s}}: \quad$ amplitude at principal frequency of fluctuations for a variable ' $r$ ' taken at location 's'

$\mathbf{f}_{\mathbf{p}}$ : $\quad$ principal frequency of inlet pressure fluctuations $(\mathrm{Hz})$

$\overline{\mathbf{T}}_{\mathbf{w} \mid \mathrm{Ref}}$ : time-averaged (mean) condensing surface temperatures that are also spatially averaged over a given length $\left({ }^{\circ} \mathrm{C}\right)$

$\overline{\mathbf{T}}_{\mathbf{w}}(\mathbf{x})$ : time-averaged (mean) condensing surface temperature at downstream location ' $\mathbf{x}$ ' $\left({ }^{\circ} \mathrm{C}\right)$

$\dot{\mathbf{M}}_{\text {in }}$ : mass flow rate of vapor supplied to condenser (g/s)

$\mathbf{h}_{\mathrm{fg}}$ : enthalpy of vaporization of the working fluid at mean inlet pressure $(\mathrm{kJ} / \mathrm{kg})$ 
$\Delta \mathbf{p}_{\mathbf{x}}:$ pressure-difference between condenser inlet and a downstream location indicated by ' $\mathrm{X}$ ' (Pa or $\mathrm{kPa})$

p: $\quad$ pressure $(\mathrm{kPa})$

$\mathbf{p}_{\text {in }}^{*}: \quad$ inlet pressure feedback control set point $(\mathrm{kPa})$

$\mathbf{V}_{\text {in }}: \quad$ cross-sectionally and temporally averaged vapor velocity at inlet $(\mathrm{m} / \mathrm{s})$

PID: proportional-integral-derivative approach of feedback control

t: $\quad$ time (seconds or minutes)

$\mathbf{T}_{\text {bath }}:$ temperature of water surrounding evaporator $\left({ }^{\circ} \mathrm{C}\right)$

TEC: thermoelectric cooler

X: distance from condenser inlet in direction of flow $(\mathrm{cm})$

$\mathbf{x}_{\mathrm{A}}: \quad$ instantaneous length of annular flow regime in condenser $(\mathrm{cm})$

$\mathbf{x}^{\star}$ : $\quad$ distance from the condenser inlet to a fixed location of interest within the annular flow regime $(\mathrm{cm})$

ठ: $\quad$ liquid film thickness $(\mu \mathrm{m})$

PC: passive condensing surface cooling condition

MC: mixed condensing surface cooling condition

N-IF: associated with a no-imposed fluctuation quasi-steady flow realization

IF: associated with a quasi-steady flow realization under externally imposed fluctuations

IF-LA: associated with low amplitude (subcritical) imposed fluctuation quasi-steady flows

IF-TA: associated with a transition flow between the low and high amplitude imposed-fluctuation responses

IF-HA: associated with high amplitude (supercritical) imposed fluctuation quasi-steady flows

\section{Additional Superscripts and Subscripts:}

in: at condenser inlet

exit: at condenser exit

cr: associated with a "critical" or threshold level of imposed fluctuation amplitude 


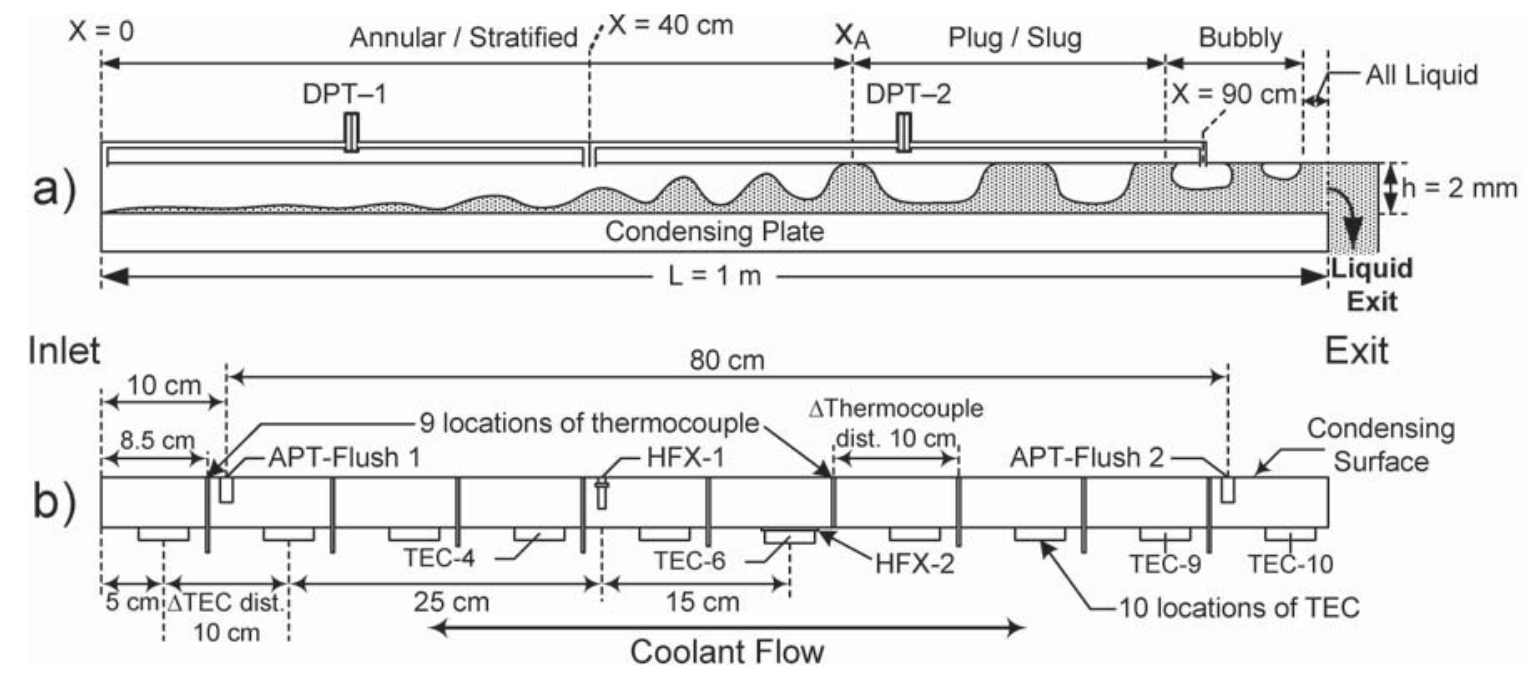

Figure 3.1. Side views of (a) test-section and (b) instrumented condensing plate.

\subsection{Terminologies used for the reported experimental investigation}

The horizontal channel flow in the test-section of Fig. 3.1a (channel height is ' $h$ ' and channel width is ' $w$ ') is the model problem for this experimental study of shear-driven flows. The flow-loop and experimental measurements described in the next section are designed to aid in these investigations. These experiments are first carried out for quasi-steady, shear-driven, and completely condensing flows under negligible imposed fluctuations (termed N-IF). These flows are effectively similar to steady flows.

The flow-variables are time-averaged over sufficiently long durations, and the resulting steady values are denoted with an over-bar. The incoming pure vapor (of FC-72 from $3 \mathrm{M}$ Corp., i.e. perfluorohexane) is at a mean inlet pressure of $\bar{p}_{\text {in }}$, mass flow rate $\overline{\mathrm{M}}_{\text {in }}$, and temperature $\bar{T}_{V \text {-in }}$. The saturation temperature associated with $\bar{p}_{\text {in }}\left(\right.$ i.e. $\left.T_{\text {sat }}\left(\bar{p}_{\text {in }}\right)\right)$ is denoted as $T_{\text {sat }}$. The incoming vapor is slightly super-heated (i.e. $\mathrm{T}_{\text {sat }} \leq \overline{\mathrm{T}}_{\mathrm{V} \text {-in }} \leq \mathrm{T}_{\text {sat }}+11^{\circ} \mathrm{C}$ ), but the effects of superheat are typically negligible over most of the condenser's length and pressure pulsations considered here. The condensing-surface's steady cooling condition is defined by the time-averaged values of the experimentally measured transient temperature profile (i.e. $\left.T_{w}(x, t)\right)$, which is denoted as $\bar{T}_{w}(x)$, where $x$ is the downstream distance from the inlet (see Fig. 3.1a). 
If the above conditions are properly chosen, the flow self-seeks an exit pressure $\bar{p}_{\text {exit }}$ and defines:

(i) a condensing-surface heat-flux variation $\overline{\mathrm{q}_{\mathrm{w}}^{\prime \prime}}(\mathrm{x})$ whose quasi-steady value at $x=40 \mathrm{~cm}$ is experimentally measured by the heat-flux meter HFX-1 in Fig. 3.1b and is denoted as $\overline{\mathrm{q}_{40 \mathrm{~cm}}^{\prime \prime}}$,

(ii) a time-averaged length of the annular zone $\bar{x}_{A}$ which is typically less than the condenser length ' $L$ ' $\left(0 \leq \bar{X}_{A} \leq L\right)$,

(iii) the steady film thickness $\bar{\delta}(\mathrm{x})$ in the annular zone (whose instantaneous values are denoted as $\delta(x, t))$, and

(iv) a quasi-steady pressure-difference $\overline{\Delta p}_{x}$ between the inlet and a downstream location at ' $x$ ' (see Fig. 3.1a) whose value at $x=40 \mathrm{~cm}$ is experimentally measured and denoted as $\overline{\Delta p}_{40 \mathrm{~cm}}$.

Another differential pressure transducer (DPT-2 in Fig. 3.1a) measures the pressure difference between $x=40 \mathrm{~cm}$ and $x=90 \mathrm{~cm}$ downstream of the condenser inlet. Together, the two differential pressure transducers yield the approximate mean and instantaneous pressure difference, $\overline{\Delta p}_{90 \mathrm{~cm}}$ and $\Delta \mathrm{p}_{90 \mathrm{~cm}}(\mathrm{t})$ respectively, between the condenser inlet and a location $90 \mathrm{~cm}$ downstream where the flow is mostly liquid. This approximates the overall pressure drop of the $100 \mathrm{~cm}$ long condenser.

The actual transient values of the above described flow variables (written as steady time-averaged values plus a time-varying part) for the negligible imposed fluctuation (N-IF) case above, as well as the imposed fluctuation (IF) cases to be described next, are denoted as: 


$$
\begin{aligned}
& \dot{M}_{i n}(t) \equiv \bar{M}_{i n}+\dot{M}_{i n}^{\prime}(t) \\
& p_{i n}(t) \equiv \bar{p}_{i n}+p_{i n}^{\prime}(t) \\
& T_{w}(x, t) \equiv \bar{T}_{w}(x)+T_{w}^{\prime}(x, t) \\
& p_{e x i t}(t) \equiv \bar{p}_{e x i t}+p_{e x i t}^{\prime}(t) \\
& \delta(x, t) \equiv \bar{\delta}(x)+\delta^{\prime}(x, t) \\
& \left.\delta(x, t)\right|_{x=40 \mathrm{~cm}} \equiv \bar{\delta}_{40 \mathrm{~cm}}+\delta_{40 \mathrm{~cm}}^{\prime}(t) \\
& x_{A}(t) \equiv \bar{x}_{A}+x_{A}^{\prime}(t) \\
& \Delta p(x, t) \equiv p(0, t)-p(x, t) \equiv \overline{\Delta p}(x)+\Delta p^{\prime}(x, t) \\
& \left.\Delta p(x, t)\right|_{x=40 \mathrm{~cm}} \equiv \Delta p_{40 \mathrm{~cm}}(t) \equiv \overline{\Delta p}_{40 \mathrm{~cm}}+\Delta p_{40 \mathrm{~cm}}^{\prime}(t) \\
& \left.\Delta p(x, t)\right|_{x=90 \mathrm{~cm}} \equiv \Delta p_{90 \mathrm{~cm}}(t) \equiv \overline{\Delta p}_{90 \mathrm{~cm}}+\Delta p_{90 \mathrm{~cm}}^{\prime}(\mathrm{t}) \\
& \mathrm{q}_{\mathrm{w}}^{\prime \prime}(\mathrm{x}, \mathrm{t}) \equiv \overline{\mathrm{q}}_{\mathrm{w}}^{\prime \prime}(\mathrm{x})+\mathrm{q}_{\mathrm{w}}^{\prime \prime}(\mathrm{x}, \mathrm{t}) \\
& \left.\mathrm{q}_{\mathrm{w}}^{\prime \prime}(\mathrm{x}, \mathrm{t})\right|_{\mathrm{x}=40 \mathrm{~cm}} \equiv \overline{\mathrm{q}}_{40 \mathrm{~cm}}^{\prime \prime \prime}+\mathrm{q}_{40 \mathrm{~cm}}^{\prime \prime}(\mathrm{t})
\end{aligned}
$$

The fluid density, $\rho$, thermal conductivity, $k$, specific heat, $C_{p}$, and viscosity, $\mu$, for either of the two phases are modeled as approximate constants. These constant values $\left\{\rho_{L}, k_{L}, C_{P \mid L}, \mu_{L}\right\}$ for the liquid phase are denoted by a subscript ' $L$ ' and the constant values $\left\{\rho_{v}, k_{v}, C_{P \mid V}, \mu_{v}\right\}$ for the vapor phase are denoted by a subscript ' $V$ '. The latent heat of vaporization at the inlet pressure $\bar{p}_{\text {in }}$ is denoted as $h_{\mathrm{fg}}$.

A reference wall temperature for $0 \leq x \leq x^{*}, x^{*}=40 \mathrm{~cm}$, is defined as $\bar{T}_{\mathrm{w} \mid \mathrm{Ref}}=\frac{1}{x^{*}} \cdot \int_{0}^{x^{*}} \bar{T}_{w}(x) \cdot d x$ and another reference temperature is defined (but not used in this paper) as $\bar{T}_{\text {w|Ref-2 }}=\frac{1}{x_{A}} \cdot \int_{0}^{x_{A}} \bar{T}_{w}(x) \cdot d x$. For results reported here, the controlling temperature difference is $\Delta \mathrm{T} \equiv \mathrm{T}_{\text {sat }}-\overline{\mathrm{T}}_{\text {w/Ref }}$. The constant liquid properties are evaluated at pressure $\bar{p}_{\text {in }}$ and temperature $\bar{T}_{\text {wiRef }}+0.5 \cdot \Delta \mathrm{T}$, and the constant vapor properties are evaluated at pressure $\bar{p}_{\text {in }}$ and temperature $T_{\text {sat }}$ (this is because effects of vapor superheat can be ignored within the condenser).

From the theoretical modeling of governing equations [36 - 38] for steady annular condensing flows, it is known that, on their non-dimensionalization, the non-dimensional 
values of all the flow variables (at non-dimensional distances $x / h, y / h$ ) are controlled and characterized by the following non-dimensional numbers:

Inlet Vapor Reynolds Number: $\quad R e_{\text {in }} \equiv \rho_{v} \cdot V_{\text {in }} \cdot h / \mu_{v}, \quad$ where $\quad V_{\text {in }} \equiv \bar{M}_{\text {in }} /\left(\rho_{v} \cdot h \cdot w\right)$

Condensate Jakob Number: $\quad$ Ja $\equiv\left(\mathrm{C}_{\mathrm{P} \mid \mathrm{L}} \cdot\left(\mathrm{T}_{\mathrm{sat}}-\mathrm{T}_{\mathrm{w} \mid \mathrm{Ref}}\right)\right) / \mathrm{h}_{\mathrm{fg}}$

Condensate Prandtl Number: $\quad \operatorname{Pr}_{\mathrm{L}} \equiv \mu_{\mathrm{L}} \cdot \mathrm{C}_{\mathrm{PIL}} / \mathrm{k}_{\mathrm{L}}$

Transverse Gravity Number: $\quad \mathrm{Fr}_{\mathrm{y}}^{-1} \equiv \mathrm{g}_{\mathrm{y}} \cdot \mathrm{h} / \mathrm{V}_{\text {in }}^{2}$

Fluid Property Ratios: $\quad \rho_{\mathrm{V}} / \rho_{\mathrm{L}}$ and $\mu_{\mathrm{v}} / \mu_{\mathrm{L}}$

Within the annular zone of the condenser, the experimental conditions are planned and conducted to have minimal impact of inlet vapor superheat (as measured by $\bar{T}_{V \text {-in }}-T_{\text {sat }}$ ) as well as the liquid's sub-cooling at the exit (which is important only over the non-annular flow regime and is measured by $\left.\mathrm{T}_{\text {sat }}-\overline{\mathrm{T}}_{\mathrm{L} \text {-exit }}\right)$. Despite this, non-dimensional values of these measures were obtained through the following non-dimensional numbers (see Table 3.2 and its caption):

$\begin{array}{ll}\text { Inlet Vapor Jakob Number: } & \mathrm{Ja}_{\mathrm{V} \text {-in }} \equiv\left(\mathrm{C}_{\mathrm{PIV}} \cdot\left(\overline{\mathrm{T}}_{\mathrm{V} \text {-in }}-\mathrm{T}_{\text {sat }}\right)\right) / \mathrm{h}_{\mathrm{fg}} \\ \text { Exit Liquid Jakob Number: } & \mathrm{Ja}_{\mathrm{L} \text {-exit }} \equiv\left(\mathrm{C}_{\mathrm{P} \mid \mathrm{L}} \cdot\left(\mathrm{T}_{\text {sat }}-\mathrm{T}_{\mathrm{L} \text {-exit }}\right)\right) / \mathrm{h}_{\mathrm{fg}}\end{array}$

The quasi-steady cooling conditions are specified by the spatially uniform or non-uniform values of the condensing-surface temperature $\bar{T}_{w}(x)$ or heat-flux $\overline{q_{w}^{\prime \prime}}(x)$. Since condensingsurface temperature $\bar{T}_{w}(x)$ equals the quasi-steady condensate temperature values $\bar{T}_{L}(x, y)$ at $y=0$, the non-dimensional condensing-surface temperature as obtained from the nondimensionalization of the governing equations is:

$\Theta(x / h) \equiv\left(\bar{T}_{w}(x)-T_{\text {sat }}\right) /\left(\bar{T}_{w \mid R e f}-T_{\text {sat }}\right)$

Because of the definition in Eq. (3.4), different condensing-surface temperature variation profiles $\overline{\mathrm{T}}_{\mathrm{w}}(\mathrm{x})$ (corresponding to different $\overline{\mathrm{T}}_{\mathrm{w} \mid \mathrm{Ref}}$ values) that lead to approximately the "same" non-uniform function $\Theta(x / h)$ are said to correspond to approximately the "same" 
cooling condition.

The investigation of the above quasi-steady flows' response to the imposition of commonly occurring large amplitude fluctuations is carried out by an experimental approach which has a "pulsator" in the flow loop that is placed upstream of the test-section in Fig. 3.2. This pulsator is connected to the flow loop through a valve $V_{P}$ (Fig. 3.2), and the level of opening of this valve controls the amplitude of the imposed pulsations. The variation of the driving motor speed controls the predominant frequency, $f_{p}$, associated with inlet pressure fluctuation $p_{i n}^{\prime}(t)$ in Eq. (3.1). The time-averaged values of the flow variables in Eq. (3.1) are obtained at a time-scale much longer than $\Delta t_{c} \equiv 1 / f_{p}$, the one associated with the imposed frequency $f_{p}$.

Furthermore, the imposed fluctuation (IF) cases are studied under conditions where the mean values $\overline{\mathrm{M}}_{\text {in }}, \overline{\mathrm{P}}_{\mathrm{in}}$, and $\mathrm{T}_{\mathrm{w}}(\mathrm{x})$ are held fixed through suitable experimental controls, but the flow is able to self-select quasi-steady values of the remaining flow variables (such as $\bar{p}_{\text {exit }}, \overline{\Delta p}_{40 \mathrm{~cm}}, \overline{\Delta \mathrm{p}}_{90 \mathrm{~cm}}, \overline{\mathrm{q}}_{40 \mathrm{~cm}}, \overline{\mathrm{x}}_{\mathrm{A}}$, and $\left.\overline{\mathrm{\delta}}_{40 \mathrm{~cm}}\right)$. These are non-dimensionalized as follows:

$$
\begin{aligned}
& \text { nd- } \bar{p}_{\text {in }} \equiv \bar{p}_{\text {in }} /\left(\rho_{\mathrm{v}} \cdot V_{\text {in }}^{2}\right) \\
& \text { nd- } \bar{p}_{\text {exit }} \equiv \bar{p}_{\text {exit }} /\left(\rho_{\mathrm{v}} \cdot v_{\text {in }}^{2}\right) \\
& \mathrm{nd}-\overline{\Delta \mathrm{p}}_{40 \mathrm{~cm}} \equiv \overline{\Delta \mathrm{p}}_{40 \mathrm{~cm}} /\left(\rho_{\mathrm{v}} \cdot \mathrm{V}_{\text {in }}^{2}\right) \\
& \mathrm{nd}-\overline{\mathrm{p}}_{90 \mathrm{~cm}} \equiv{\overline{\Delta \mathrm{p}_{90 \mathrm{~cm}}}} /\left(\rho_{\mathrm{v}} \cdot \mathrm{V}_{\mathrm{in}}^{2}\right) \\
& \mathrm{nd}-\overline{\mathrm{q}}_{40 \mathrm{~cm}} \equiv\left(\overline{\mathrm{q}_{40 \mathrm{~cm}}^{\prime \prime}} \cdot \mathrm{h}\right) /\left(\mathrm{k}_{\mathrm{L}} \cdot\left(\mathrm{T}_{\mathrm{sat}}-\overline{\mathrm{T}}_{40 \mathrm{~cm}}\right)\right) \\
& n d-\bar{x}_{A} \equiv \bar{x}_{A} / h \\
& \mathrm{nd}-\bar{\delta}_{40 \mathrm{~cm}} \equiv \bar{\delta}_{40 \mathrm{~cm}} / \mathrm{h}
\end{aligned}
$$

These quasi-steady flows are termed pulsatile when the dominant amplitude of mass flow rate fluctuations $\dot{M}_{\text {in }}^{\prime}(t)$ with respect to $\overline{\mathrm{M}}_{\text {in }}$ (or dominant amplitude of pressure-difference fluctuations $\Delta \mathrm{p}_{40 \mathrm{~cm}}^{\prime}(\mathrm{t})$ with respect to $\overline{\Delta \mathrm{p}}_{40 \mathrm{~cm}}$ ) is significant (say $>0.5$ ). These conditions occur even for low inlet-pressure fluctuation amplitudes (say $p_{i n}^{\prime}(t)$ in the range of $40-300 \mathrm{~Pa}$ ) relative to the mean inlet pressure $\bar{p}_{\text {in }}\left(\right.$ say $\bar{p}_{\text {in }}$ in the range of 
$100-200 \mathrm{kPa})$.

The impact of the imposed pressure fluctuation $p_{i n}^{\prime}(t)$ on shear-driven flows (because of the sensitive coupling between vapor and liquid motion) is such that both the quasi-steady values of the flow variables (those that are not actively fixed) as well as their superposed time-varying fluctuations' amplitudes and frequencies (e.g. $\Delta \mathrm{p}_{40 \mathrm{~cm}}^{\prime}(\mathrm{t}), \mathrm{q}_{40 \mathrm{~cm}}^{\prime \prime \prime}(\mathrm{t})$, etc.) are significantly affected under appropriate conditions.

To characterize the amplitudes of the measured fluctuations, the amplitude-frequency spectrums of the Fast Fourier Transform (FFT, see [39]) of the measured variables are analyzed to determine their predicted values at the pulsator's driving frequency, $f_{p}$. The time records of these dynamically measured variables were obtained in several $(16 \pm 2)$ separate records, each of 5 second duration. After appropriate data processing, the FFT magnitudes of each of these records were used to determine the representative average driving frequency and its corresponding averaged amplitudes of the measured variables. Some of these fluctuation amplitudes, as obtained from suitable dynamic measurements and data processing, are denoted as:

$a_{p-\text { in }} \equiv$ amplitude $\left(\right.$ at $\left.f=f_{p}\right)$ in the FFT of $\left.p_{\text {in }}^{\prime}(t)\right|_{t \rightarrow f}$

$a_{p \text {-exit }} \equiv$ amplitude $\left(\right.$ at $\left.f=f_{p}\right)$ in the FFT of $\left.p_{\text {exit }}^{\prime}(t)\right|_{t \rightarrow f}$

$a_{\Delta p-40 \mathrm{~cm}} \equiv$ amplitude $\left(\right.$ at $\left.f=f_{p}\right)$ in the FFT of $\left.\Delta p_{40 \mathrm{~cm}}^{\prime}(t)\right|_{t \rightarrow f}$

$a_{q^{\prime \prime}-40 \mathrm{~cm}} \equiv$ amplitude $\left(\right.$ at $\left.f=f_{p}\right)$ in the FFT of $\left.q_{40 \mathrm{~cm}}^{\prime \prime}(t)\right|_{t \rightarrow f}$

In the annular regime of the above-described pulsatile flows (imposed fluctuation IF cases) and over the short time-scales $\left(\Delta t_{c} \equiv 1 / f_{p}\right)$ of interest, the mean and fluctuating variables are governed by the unsteady annular flow equations (see [40]). A proper non-dimensionalization of these incompressible equations shows that, besides the non-dimensional numbers defined in Eq. (3.2) and the affected mean flow variables defined in Eq. (3.5), additional well-known non-dimensional numbers representing the amplitude $a_{\Delta p-x^{*}}$, distance $x^{*}$ (in this paper, $x^{*}=40 \mathrm{~cm}$ ), and frequency $f_{p}($ termed 
Strouhal number St) are also important. These additional numbers are:

$$
\begin{array}{ll}
\text { nd- } a_{\Delta p-x^{*}} & \equiv a_{\Delta p-x^{*}} /\left(\rho_{v} \cdot V_{\text {in }}^{2}\right) \\
\text { nd- } x^{*} & \equiv x^{*} / h \\
\text { St } & \equiv \mathrm{f}_{\mathrm{p}} \cdot \mathrm{h} / \mathrm{V}_{\text {in }}
\end{array}
$$

Since the fluctuating variables' representative amplitudes $\left(a_{p-i n}, a_{\Delta p-40 \mathrm{~cm}}, a_{q^{\prime \prime}-40 \mathrm{~cm}}, a_{\delta-40 \mathrm{~cm}}\right.$, etc.) are also affected, their non-dimensional values are defined as (according to the convention of Eq. (3.5)):

$$
\begin{aligned}
& \text { nd- } a_{p-\text { in }} \equiv a_{p-\text { in }} /\left(\rho_{v} \cdot V_{\text {in }}^{2}\right) \\
& \text { nd- } a_{\Delta p-40 \mathrm{~cm}} \equiv \mathrm{a}_{\Delta \mathrm{p}-40 \mathrm{~cm}} /\left(\rho_{\mathrm{v}} \cdot \mathrm{V}_{\text {in }}^{2}\right) \\
& n d-a_{q^{\prime \prime}-40 \mathrm{~cm}} \equiv \mathrm{a}_{\mathrm{q}^{\prime \prime}-40 \mathrm{~cm}} \cdot \mathrm{h} /\left(\mathrm{k}_{\mathrm{L}} \cdot\left(\mathrm{T}_{\mathrm{sat}}-\overline{\mathrm{T}}_{40 \mathrm{~cm}}\right)\right) \\
& n d-a_{\delta-40 \mathrm{~cm}} \equiv a_{\delta-40 \mathrm{~cm}} / \mathrm{h}
\end{aligned}
$$

The transient compressibility effects in the vapor phase could be important as they are known to be important in determining the time-varying vapor mass flow rate at locations upstream of the condenser (see [41]). An estimated non-dimensional measure of the importance of transient compressibility effects (which accounts for relevant effects of forward-and-backward moving acoustic waves) in a given volume and over small time-scales (of the order of $1 / f_{p}$ ) of interest here, as discussed in section 3.5, is shown in [41] to be adequately characterized by a transient compressibility related non-dimensional (TCND) parameter defined as $\operatorname{TCND}_{\text {Total }}=2 \cdot \pi \cdot \frac{x^{*}}{h} \cdot\left(\frac{a_{p-i n}+0.5 \cdot a_{\Delta p-40 m}}{\rho_{v^{V}} \cdot c_{0}^{2}}\right)$, where $c_{0}$ is speed of sound in the saturated vapor and $x^{*} / h=200$ for the vapor volume between $x=0 \mathrm{~cm}$ and $x=40 \mathrm{~cm}$ in the test-section.

For low amplitude $a_{p-i n}$ imposition, it is expected that TCND $_{\text {Total }}$ values will be small, and interfacial wave effects are adequately modeled by assuming the vapor is incompressible. However, for high amplitude $\mathrm{a}_{\mathrm{p} \text {-in }}$ impositions, the transient compressibility parameters TCND $_{\text {Total }}$ may be used (see [41]) to determine whether there are significant interactions 
between compressibility effects and interfacial waves. This is the reason why the values of $\mathrm{TCND}_{\text {Total }}$ are reported in Table 3.2. Since the non-dimensional frequency $F_{P}=\left(x^{*} / h\right) \cdot S t$ appears in the definition of $T C N D_{\text {Total }}$ it is considered a better measure (compared to $S t$ ) of non-dimensional frequency $f_{P}$ with regard to its relevance in modeling effects of compressibility. Therefore $F_{P}$ has been reported in Table 3.2 and the relevant Figures.

The experimental data-matrix in physical variables is listed in Table 3.1; and its values in non-dimensional terms, along with some key experimental results, are listed in Table 3.2. 
Table 3.1.

Representative flow conditions for the cases used in the data-matrix of Fig. 3.14. The saturation temperature of the The sound speed $c_{0}$ showed little variation and was between 89.82 and $89.83 \mathrm{~m} / \mathrm{s}$.

\begin{tabular}{|c|c|c|c|c|c|c|c|c|c|c|c|c|c|}
\hline \multirow[t]{2}{*}{ Case } & $\begin{array}{l}\text { Cooling } \\
\text { Condition }\end{array}$ & Type & $\begin{array}{l}\bar{M} \\
\mathrm{~g} / \mathrm{s}\end{array}$ & $\begin{array}{l}\bar{p}_{\text {in }} \\
\mathrm{kPa}\end{array}$ & $\begin{array}{l}V_{\text {in }} \\
\mathrm{m} / \mathrm{s}\end{array}$ & $\begin{array}{l}f_{p} \\
\mathrm{~Hz}\end{array}$ & $\begin{array}{l}a_{p-\text { in }} \\
\mathrm{kPa} \\
\end{array}$ & $\begin{array}{c}a_{p \text {-exit }} \\
\mathrm{kPa}\end{array}$ & $\begin{array}{c}\overline{\Delta p}_{40 \mathrm{~cm}} \\
\mathrm{~Pa}\end{array}$ & $\begin{array}{c}a_{\triangle p-40 \mathrm{~cm}} \\
\mathrm{~Pa}\end{array}$ & $\begin{array}{c}\overline{\Delta p}_{90 \mathrm{~cm}} \\
\mathrm{~Pa}\end{array}$ & $\begin{array}{c}a_{\Delta p-90 \mathrm{~cm}} \\
\mathrm{~Pa}\end{array}$ & $\begin{array}{l}\bar{p}_{\text {exit }} \\
\mathrm{kPa}\end{array}$ \\
\hline & Passive & N-IF & 1.1982 & 142.02 & 2.14 & - & -- & (-- & $\begin{array}{ll}131 \\
\end{array}$ & (-- & 188 & -- & 141.85 \\
\hline P09 & Passive & IF-LA & 1.1999 & 142.01 & 2.14 & 3.9 & 0.306 & 0.368 & 115 & 117 & 237 & 421 & 141.69 \\
\hline P10 & Passive & IF-TA & 1.2010 & 142.00 & 2.15 & 3.9 & 0.590 & 0.613 & 122 & 190 & - & - & 141.65 \\
\hline P11 & Passive & IF-HA & 1.2005 & 142.00 & 2.15 & 3.8 & 0.840 & 0.820 & 133 & 282 & - & - & 141.63 \\
\hline P12 & Passive & IF-HA & 1.1972 & 141.99 & 2.14 & 3.8 & 1.116 & 1.007 & 149 & 365 & - & - & 141.63 \\
\hline P13 & Passive & IF-HA & 1.1985 & 142.02 & 2.14 & 3.8 & 1.511 & 1.348 & 164 & 518 & - & - & 141.65 \\
\hline P14 & Passive & IF-HA & 1.1975 & 141.99 & 2.14 & 3.9 & 2.412 & 2.216 & 137 & 885 & - & - & 141.61 \\
\hline P07 & Passive & IF-LA & 1.1959 & 142.00 & 2.14 & 10.2 & 0.098 & 0.039 & 112 & 179 & 177 & 252 & 141.80 \\
\hline P05 & Passive & IF-TA & 1.1990 & 142.00 & 2.14 & 10.2 & 0.281 & 0.117 & 121 & 434 & 192 & 653 & 141.70 \\
\hline P04 & Passive & IF-HA & 1.2016 & 141.99 & 2.15 & 10.2 & 0.580 & 0.362 & 110 & 771 & - & - & 141.63 \\
\hline P03 & Passive & IF-HA & 1.2021 & 141.99 & 2.15 & 10.2 & 0.773 & 0.522 & 100 & 924 & -- & - & 141.59 \\
\hline P02 & Passive & IF-HA & 1.1997 & 141.99 & 2.14 & 10.1 & 1.145 & 0.936 & 129 & 1230 & - & - & 141.68 \\
\hline C15 & Mixed & IF-LA & 1.2022 & 141.99 & 2.15 & 10.5 & 0.280 & 0.125 & 198 & 427 & 291 & 611 & 141.88 \\
\hline C13 & Mixed & IF-TA & 1.2024 & 142.01 & 2.15 & 10.5 & 0.400 & 0.214 & 223 & 569 & 301 & 819 & 141.87 \\
\hline C11 & Mixed & IF-HA & 1.2019 & 142.01 & 2.15 & 10.4 & 0.564 & 0.368 & 231 & 744 & 360 & 1085 & 141.86 \\
\hline $\mathrm{CO9}$ & Mixed & IF-HA & 1.2002 & 141.99 & 2.15 & 10.4 & 0.863 & 0.680 & 180 & 944 & - & - & 141.86 \\
\hline $\mathrm{CO5}$ & Mixed & IF-HA & 1.1994 & 142.00 & 2.14 & 10.5 & 1.811 & 1.997 & 113 & 1335 & - & - & 141.91 \\
\hline C19 & Mixed & IF-LA & 1.2039 & 141.99 & 2.15 & 16.2 & 0.067 & 0.034 & 174 & 192 & 269 & 269 & 141.97 \\
\hline $\mathrm{C} 21$ & Mixed & IF-TA & 1.2021 & 141.99 & 2.15 & 16.2 & 0.472 & 0.281 & 245 & 678 & 271 & 1003 & 141.90 \\
\hline $\mathrm{C} 23$ & Mixed & IF-HA & 1.1819 & 141.99 & 2.11 & 16.3 & 1.137 & 1.067 & 231 & 1056 & - & - & 141.90 \\
\hline NF1 & Mixed & N-IF & 1.2023 & 142.00 & 2.15 & - & - & - & 187 & - & 283 & - & 141.97 \\
\hline
\end{tabular}

Table 3.2.

Non-dimensional representation of cases in Table 3.1. The density ratio $\rho_{V} / \rho_{L}$ and viscosity ratio $\mu_{V} / \mu_{L}$ are not rep variations with $0.01155<\rho_{V} / \rho_{L}<0.01157$ and $0.02579<\mu_{V} / \mu_{L}<0.02599$. Similarly, inlet vapor Jakob numbe range of $0.00-0.05$.

\begin{tabular}{|c|c|c|c|c|c|c|c|c|c|c|c|c|c|c|}
\hline Case & $\begin{array}{l}\text { Cooling } \\
\text { Condition }\end{array}$ & Type & $\mathrm{Re}_{\text {in }}$ & $n d-\bar{p}_{\text {in }}$ & $F_{p}$ & $\mathrm{Ja} / \mathrm{Pr}_{\mathrm{L}}$ & $n d-a_{p-i n}$ & $n d-a_{p-e x i t}$ & $\mathrm{nd}-\overline{\Delta p}_{40 \mathrm{~cm}}$ & $n d-a_{\Delta p-40 \mathrm{~cm}}$ & $\mathrm{nd}-\overline{\Delta p}_{90 \mathrm{~cm}}$ & $n d-a_{\Delta p-90 c m}$ & $\mathrm{Ja}_{\text {L-exit }}$ & $\mathrm{nd}-\overline{\mathrm{q}}^{\prime \prime}{ }_{40 \mathrm{~cm}} \mathrm{nc}$ \\
\hline NF3 & Passive & $\mathrm{N}-\mathrm{IF}$ & 7031 & 161660 & ב-.. & 0.02190 & (-... & $\ldots$ & 1.53 & $\ldots$ & 2.20 & $\ldots$ & 0.275 & 7.7 \\
\hline P09 & Passive & IF-LA & 7042 & 1655 & 0.72 & 0.02146 & 3.56 & 4.29 & 1.34 & 1.37 & 2.77 & 4.91 & 0.279 & 8.1 \\
\hline P10 & Passive & IF-TA & 7048 & 1652 & 0.72 & 0.02119 & 6.87 & 7.13 & 1.42 & 2.22 & -.. & ... & 0.280 & 9.0 \\
\hline $\mathrm{P} 11$ & Passive & IF-HA & 7045 & 1654 & 0.71 & 0.02106 & 9.79 & 9.55 & 1.55 & 3.28 & -.. & -.. & 0.283 & 13.3 \\
\hline $\mathrm{P} 12$ & Passive & IF-HA & 7025 & 1663 & 0.71 & 0.02085 & 13.07 & 11.79 & 1.74 & 4.27 & -.. & $\ldots$ & 0.284 & 20.0 \\
\hline $\mathrm{P} 13$ & Passive & $\mathrm{IF}-\mathrm{HA}$ & 7033 & 1660 & 0.71 & 0.02047 & 17.65 & 15.75 & 1.91 & 6.05 & -.. & $\ldots$ & 0.290 & 32.7 \\
\hline P14 & Passive & IF-HA & 7028 & 1662 & 0.72 & 0.01962 & 28.23 & 25.94 & 1.61 & 10.36 & -.. & $\ldots$ & 0.303 & 55.0 \\
\hline P07 & Passive & IF-LA & 7018 & 1666 & 1.91 & 0.02156 & 1.15 & 0.45 & 1.31 & 2.10 & 2.07 & 2.96 & 0.270 & 7.7 \\
\hline P05 & Passive & IF-TA & 7036 & 1658 & 1.91 & 0.02122 & 3.29 & 1.36 & 1.41 & 5.07 & 2.24 & 7.62 & 0.272 & 11.1 \\
\hline P04 & Passive & IF-HA & 7052 & 1650 & 1.90 & 0.02049 & 6.74 & 4.21 & 1.27 & 8.96 & ... & ... & 0.278 & 27.4 \\
\hline P03 & Passive & IF-HA & 7054 & 1649 & 1.90 & 0.02010 & 8.98 & 6.06 & 1.17 & 10.73 & ... & -.. & 0.185 & 36.7 \\
\hline P02 & Passive & IF-HA & 7040 & 1656 & 1.89 & 0.01919 & 13.36 & 10.91 & 1.50 & 14.34 & -.. & ... & 0.279 & 47.2 \\
\hline C15 & Mixed & IF-LA & 7055 & 1649 & 1.95 & 0.02011 & 3.25 & 1.45 & 2.30 & 4.95 & 3.38 & 7.09 & 0.298 & 9.3 \\
\hline $\mathrm{C} 13$ & Mixed & IF-TA & 7056 & 1649 & 1.95 & 0.01998 & 4.64 & 2.49 & 2.59 & 6.61 & 3.49 & 9.51 & 0.301 & 11.5 \\
\hline C11 & Mixed & IF-HA & 7053 & 1650 & 1.94 & 0.01986 & 6.56 & 4.27 & 2.68 & 8.64 & 4.18 & 12.60 & 0.305 & 18.8 \\
\hline $\mathrm{C} 09$ & Mixed & IF-HA & 7043 & 1654 & 1.94 & 0.01960 & 10.06 & 7.92 & 2.10 & 11.00 & ... & -.. & 0.321 & 35.0 \\
\hline $\mathrm{C} 05$ & Mixed & IF-HA & 7039 & 1657 & 1.95 & 0.01884 & 21.13 & 23.29 & 1.32 & 15.58 & ... & -.. & 0.339 & 52.2 \\
\hline $\mathrm{C} 19$ & Mixed & IF-LA & 7065 & 1644 & 3.01 & 0.02025 & 0.78 & 0.39 & 2.02 & 2.22 & 3.11 & 3.11 & 0.295 & 8.3 \\
\hline $\mathrm{C} 21$ & Mixed & IF-TA & 7054 & 1649 & 3.02 & 0.01993 & 5.49 & 3.27 & 2.85 & 7.88 & 3.14 & 11.65 & 0.299 & 11.8 \\
\hline $\mathrm{C} 23$ & Mixed & IF-HA & 6936 & 1706 & 3.08 & 0.01938 & 13.66 & 12.82 & 2.78 & 12.69 & $\ldots$ & ... & 0.336 & 38.5 \\
\hline NF1 & Mixed & $\mathrm{N}-\mathrm{IF}$ & 7056 & 1649 & ... & 0.02064 & $\ldots$ & $\ldots$ & 2.18 & $\ldots$ & 3.28 & $\ldots$ & 0.304 & 8.6 \\
\hline
\end{tabular}





\subsection{Experimental set-up}

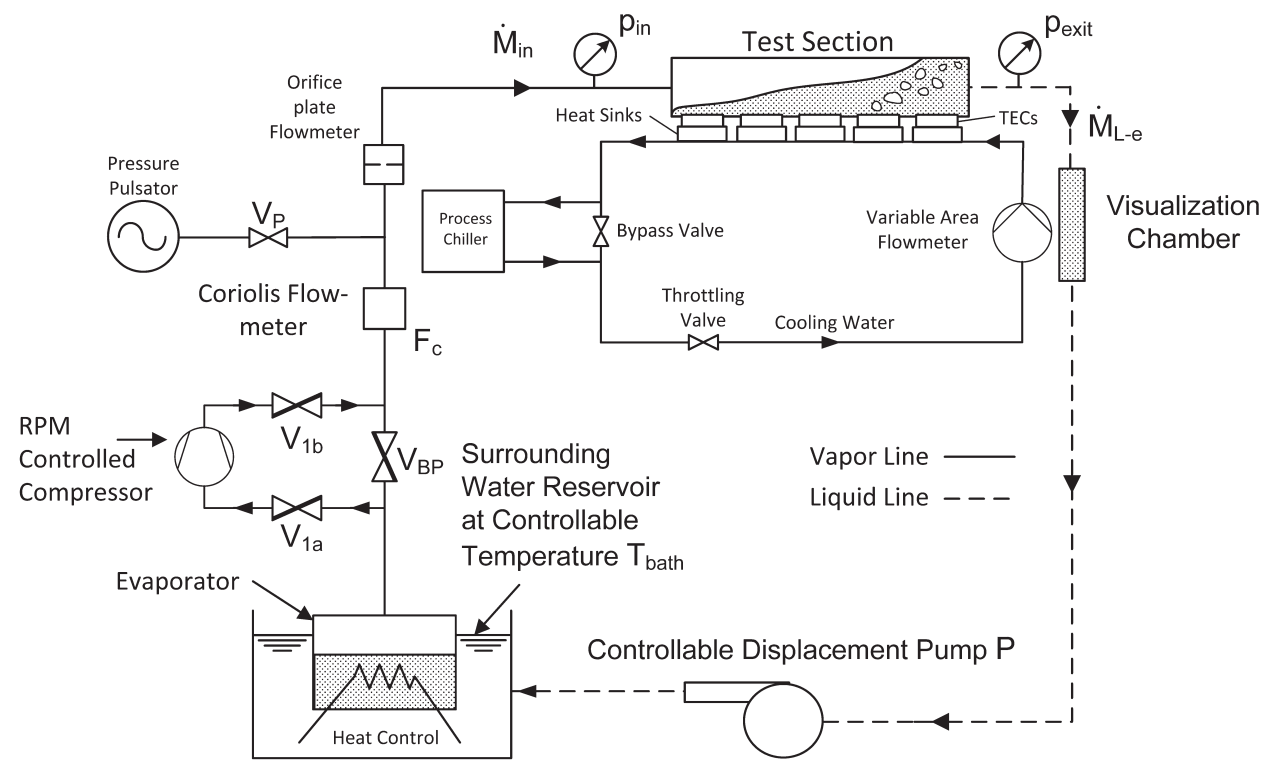

Figure 3.2. Schematic of the experimental flow loop used to investigate complete condensation.

\subsubsection{Description}

Fully condensing flows of FC-72 vapor are investigated in a horizontal, rectangular cross-section ( $2 \mathrm{~mm}$ gap height and $15 \mathrm{~mm}$ width) duct of 1 meter length (hereafter termed "test-section"). Its horizontal condensing-surface area $(15 \mathrm{~mm} \times 1 \mathrm{~m})$ is the top of a $12.7 \mathrm{~mm}$ thick stainless steel plate (which exhibits non-negligible thermal inertia under transient conditions). The channel's top and side surfaces are made of a thick transparent material (lexan), which is covered with insulation that can be removed to allow for flow visualization. Transport and thermodynamic fluid properties of FC-72 were obtained from 3M Corporation. This choice of fluid is for safety of operations (e.g. non-flammable and boiling temperature of $56.6^{\circ} \mathrm{C}$ ) under laboratory conditions at a university and for convenient pressure and temperature ranges for commercially available pressure transducers.

A 0 - 300 watt electric ring heater inside the evaporator/boiler ("heat control" of Fig. 3.2) causes the working fluid (FC-72) to evaporate and/or experience nucleate boiling near the heater surface. The vapor mass flow rate out of the evaporator, $\dot{M}_{i n}$, is fed 
into the test-section. Its mean value is measured by a Coriolis mass flow meter, $\mathrm{F}_{\mathrm{C}}$, and brought to a steady set-point value by a feedback-controlled heating of this ring heater through a PID control implemented in LabVIEW. A description of the speed-controlled compressor and its use may be found in [30 - 31]. It was not used (and the associated bypass valve, $\mathrm{V}_{\mathrm{BP}}$, was fully open) in the experiments reported here.

The flow-loop in Fig. 3.2 has three independent feedback control strategies that can fix quasi-steady values of the inlet mass flow rate $\overline{\mathrm{M}}_{\text {in }}$, condensing-surface cooling conditions, and inlet (or exit) pressure.

Mean inlet mass flow rate, $\overline{\mathrm{M}}_{\text {in }}$, is fixed through active feedback control of the power input to the electric heater inside the evaporator/boiler. Evaporator pressure is stabilized using the surrounding water reservoir temperature, $\mathrm{T}_{\text {bath }}$.

Condensing-surface temperature $\bar{T}_{w}(x)$ is obtained for fully specified quasi-steady cooling approaches that result from a specified steady temperature of the coolant water (where it first approaches the condensing plate's heat sinks) and its specified steady flow rate (0 - 17 liters/minute). For the experiments reported here, the water flow rate was between 7.3 and 8.1 liters/minute, and the temperature of the cooling water at the exit of the chiller was $15-16{ }^{\circ} \mathrm{C}$ for the "passive cooling" condition cases and around $24{ }^{\circ} \mathrm{C}$ for the mixed cooling condition cases.

The flow of coolant water in Fig. 3.2 (see also [24]) is supplied with the help of a commercially available process chiller of $5040 \mathrm{BTU} / \mathrm{hour}\left(\right.$ at $65^{\circ} \mathrm{F}$ ) cooling capacity $\left(1477 \mathrm{~W}\right.$ at $\left.18.3^{\circ} \mathrm{C}\right), 4$ gallons/minute (at 50 psi) water pump rating (15 liters/minute at $345 \mathrm{kPa}$ ), and thermostat control in increments of $1^{\circ} \mathrm{C}$. An adjustable bypass valve connecting the chiller discharge and suction ports, coupled with a throttling valve (see the simplified "cooling water" loop of Fig. 3.2), allowed the cooling water flow rate to be varied within the range of $0-17$ liters/minute over discharge pressures within the range of $0-275 \mathrm{kPa}$ gage. 
This cooling approach may further be specified depending on whether or not several separately controlled thermoelectric coolers (TECs) are used (see Fig. 3.1b). These TECs are on the bottom surface of the condensing-plate (Figs. 3.1b, 3.2) and reject heat into the flow of cooling water underneath. When the TECs are not used, heat flows through them by conduction into the heat sinks. For this paper, the cooling condition in which none of the TECs are used will be compared to the cooling condition in which four of the TECs are used. When TECs are used, two of the TECs are used to fix the wall temperatures at $38.5 \mathrm{~cm}$ and $58.5 \mathrm{~cm}$ downstream of the condenser inlet through PID feedback control and two TECs near the exit of the condenser are used at a fixed high power level to ensure that the fluid completely condenses before the exit of the test-section.

Either the mean exit pressure, $\bar{p}_{\text {exit }}$ or the mean inlet pressure, $\bar{p}_{\text {in }}$, can be held fixed by active feedback control of the controllable displacement pump P. For the experiments reported in this paper, $\bar{p}_{\text {in }}$ is held fixed, while $\bar{p}_{\text {exit }}$ is left free to seek its required value.

To test the hypothesis that the changes in the mean test-section pressure-difference and annular regime heat-flux are affected by the amplitude and frequency content of the superposed periodic pressure fluctuations at the test-section inlet, a pressure pulsator was introduced and located downstream of flow meter $F_{c}$ in Fig. 3.2. This pulsator (a frequency-controlled diaphragm compressor, which is used after removing the valves between its suction and compression chambers) cannot change the mean flow rate but is able to provide an independent control on pressure and mass flow rate fluctuations present at the test-section inlet. The frequency of the pulsator's imposed pressure fluctuations is controlled by the pulsator motor speed, and their representative amplitude is controlled by the throttling valve $V_{p}$. The fluctuational component of the mass flow rate is obtained (see [41]) by suitable dynamic pressure-difference measurements across an orifice-plate flow meter of our own design (see Fig. 3.2). 


\subsubsection{I nstrumentation}

Kulite flush-type absolute pressure transducers are used in the test-section at locations $10 \mathrm{~cm}$ and $90 \mathrm{~cm}$ downstream of the test-section inlet. Their accuracies, after calibration, are $\pm 0.7 \mathrm{kPa}$. A total of four high-accuracy pressure transducers from Omega Engineering are used. These measure upstream and downstream absolute pressures for the orifice meter and the test-section condenser. Their accuracies, after calibration, are $\pm 0.2 \mathrm{kPa}$ for the test-section transducers and $\pm 0.5 \mathrm{kPa}$ for the transducers at the orifice meter in Fig. 3.2. The accuracies of the other pressure transducers in the system are approximately within $\pm 0.6 \mathrm{kPa}$.

The differential pressure transducer DPT-1 (see Fig. 3.1a), used to measure the pressure-difference, $\Delta p_{40 \mathrm{~cm}}$, across the first $40 \mathrm{~cm}$ of the test-section, is from Validyne Inc. It is a variable-reluctance-type transducer, and its accuracy, after calibration, is $\pm 20 \mathrm{~Pa}$. The second differential pressure transducer DPT-2 (also from Validyne Inc., see Fig. 3.1a) measures pressure-difference across most of the remainder of the test-section length. When added, the readings from DPT-1 and DPT-2 yield the total pressure-difference, $\Delta p_{90 \mathrm{~cm}}$, between the test-section inlet and a location $90 \mathrm{~cm}$ downstream of the inlet, where the vapor has completely or very nearly completely condensed. For most of the high amplitude cases in Tables 3.1 and 3.2, the pressure peaks across DPT-2 were outside of its measureable range, so no values of $\overline{\Delta p}_{90 \mathrm{~cm}}$ or $\mathrm{a}_{\Delta \mathrm{p}-90 \mathrm{~cm}}$ are reported for these cases. The main difference between $\Delta \mathrm{p}_{40 \mathrm{~cm}}$ and $\Delta \mathrm{p}_{90 \mathrm{~cm}}$ is that $\Delta \mathrm{p}_{40 \mathrm{~cm}}$ is the pressure-difference where the flow regime is only annular, but $\Delta \mathrm{p}_{90 \mathrm{~cm}}$ is the pressure-difference inclusive of all the flow regimes in the test-section. The accuracy of $\Delta \mathrm{p}_{90 \mathrm{~cm}}$ is approximately $\pm 50 \mathrm{~Pa}$.

Temperatures are measured by T-type thermocouples with accuracies, after calibration, lying within $\pm 1^{\circ} \mathrm{C}$. The heat-flux meter HFX-1 (from Vatell Corporation) in Fig. 3.1b has an accuracy of approximately $\pm 7.2 \%$ of its reading, in $\mathrm{W} / \mathrm{cm}^{2}$, and an approximate range of $0-10 \mathrm{~W} / \mathrm{cm}^{2}$ when used with our existing amplifier and data acquisition system. The mean mass flow rate measured from the Coriolis Meter, $F_{c}$, in Fig. 3.2 is 
accurate up to $\pm 0.35 \%$ of the measured flow, or within $\pm 0.0042 \mathrm{~g} / \mathrm{s}$ for the flow rate $(1.2 \mathrm{~g} / \mathrm{s})$ investigated here. Only the quasi-steady mean mass flow rate can be obtained from the Coriolis meter $\left(F_{c}\right)$ readings as the device cannot resolve the frequencies of interest for dynamic data acquisition.

The orifice-plate flow meter indicated in Fig. 3.2 is our own design, and its dynamic pressure-difference signal, in conjunction with computational fluid dynamic analysis and suitable calibration experiments, can yield approximate estimates of the time-varying values of mass flow rates fluctuating around a steady mean (see Eq. 3.1) at the exit of the orifice meter. The implementation of the measurement principle developed by us [41] for this purpose needs additional work to relate the predicted time-varying mass flow rate at the exit of the orifice-meter to the time-varying mass flow rate at the inlet of the test-section. For this reason, only one rough estimate of the time-varying mass flow rate at the test-section inlet is presented here (see Fig. 3.3). Despite this, the result in Fig. 3.3 is sufficient to demonstrate the fact that (for the high amplitude imposed pulsation cases) the amplitude of mass flow rate fluctuations is significant compared to its mean value. Forthcoming experiments will use an improved method (to be reported elsewhere) which is expected to yield more accurate results for mass flow rate pulsations at both the exit of the orifice meter and the inlet of the test-section. The accuracy of the flow amplitude in Fig. 3.3 is approximately $\pm 40 \%$.

For typical reporting of mean quasi-steady data of all variables over minutes to hours, the National Instruments (NI) data acquisition system is used, and data are acquired at approximately 1.1 second intervals. Some of these variables' dynamic data are acquired every $0.5 \mathrm{~ms}$ over occasional $5 \mathrm{~s}$ intervals. Together, the two rates of data acquisition (DAQ rates) reliably yield the signals' frequency content over 0 - $1000 \mathrm{~Hz}$. The DAQ devices used to acquire data at $1.1 \mathrm{~s}$ intervals and run the feedback controls are NI's PCI-6251 card, used with their SCXI-1000 chassis, containing one SCXI-1102 module, one SCXI-1102B module, and two SCXI-1124 modules. The thermocouples are all run through the SCXI-1102 module. The pressure, flow, and heat-flux transducers are all run 
through the SCXI-1102B module. The TECs $(0-20 \mathrm{~mA})$, compressor $(0-10 \mathrm{~V})$, and pulsator $(0-10 \mathrm{~V})$ output signals are run through the SCXI-1124 modules. The dynamic data are all acquired using the NI USB-6211 DAQ platform. The electrical signal output for control of pumps ( $0-10 \mathrm{~V}$ analog) and relays ( $5 \mathrm{~V}$ variable width and frequency pulse train to vary the electrical power supplied to the Heat Control in Fig. 3.2) are also run through the USB-6211 system, updated at 1.1 second intervals. The still pictures and video (Videos 3-1 - 3-3 in the accompanying data disc) are taken with a Nikon Coolpix P500 camera that is mounted on a suitably located track.

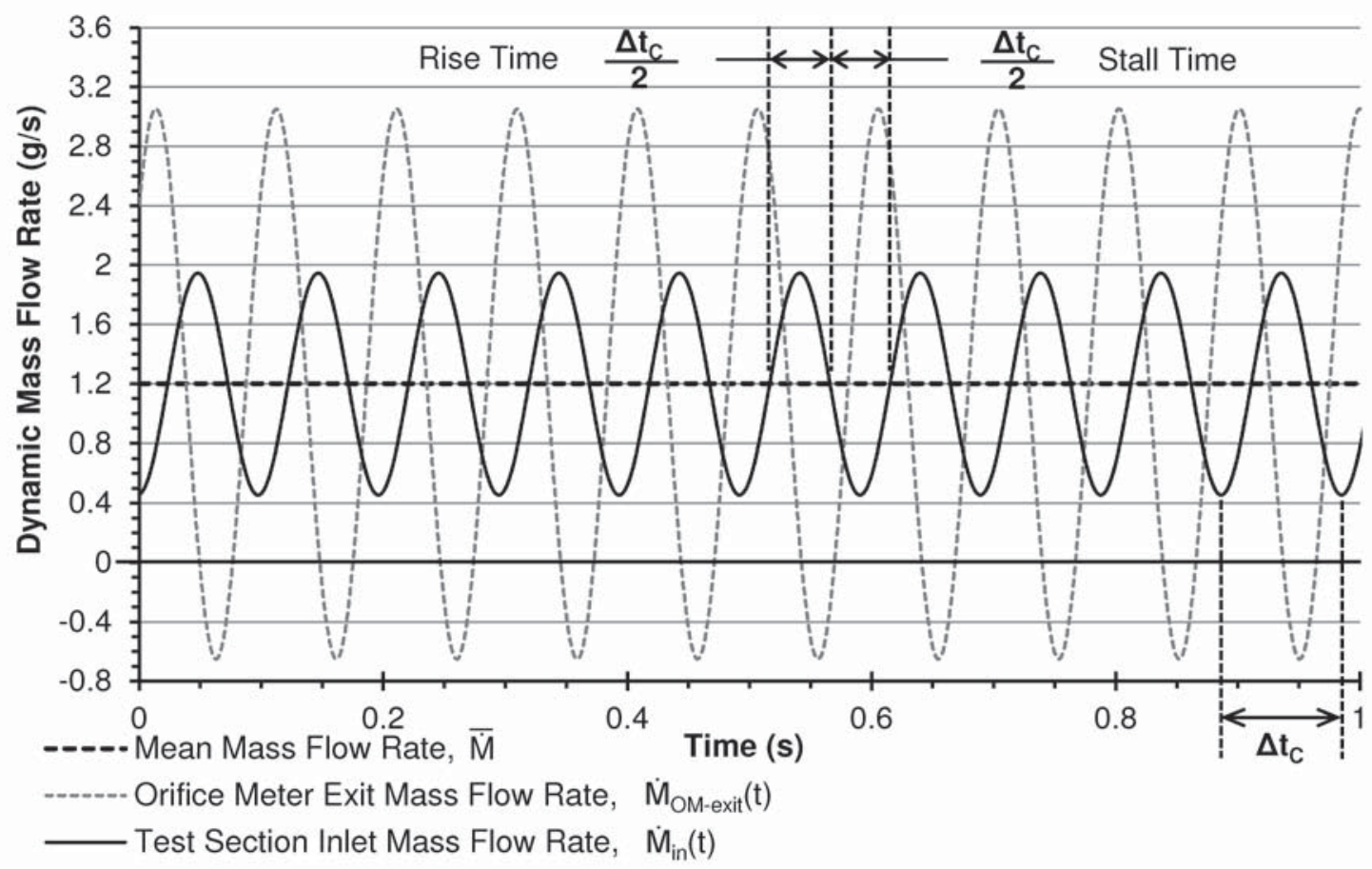

Figure 3.3. The predicted/estimated ([41]) values of the time-varying mass flow rates $\dot{M}_{O M-\text { exit }}(t)$ at the orifice meter exit and $\dot{M}_{i n}(t)$ at the test-section inlet. The data was taken from the case P02 $\left(f_{P}=10.1 \mathrm{~Hz}\right)$ in Table 3.1. 


\subsubsection{Cooling conditions and procedure}

The condensing surface's cooling approaches (which define its thermal boundary condition) investigated here consist of a combination of the following:

(i) Coolant water flows through heat sinks under the $12.7 \mathrm{~mm}$ thick condensing plate at a controlled steady flow rate $\left(0.46 \mathrm{~m}^{3} / \mathrm{hr}\right)$ and inlet temperature $\left(24^{\circ} \mathrm{C}\right)$.

(ii) Two thermoelectric coolers, namely TEC-4 and TEC-6 (see Fig. 3.1b), removing heat from approximate effective areas of $30 \mathrm{~cm} \leq x \leq 40 \mathrm{~cm}$ and $50 \mathrm{~cm} \leq \mathrm{x} \leq 60 \mathrm{~cm}$ respectively, are PID controlled at $45^{\circ} \mathrm{C}$ using the thermocouples at $x=38.5 \mathrm{~cm}$ and $x=48.5 \mathrm{~cm}$ respectively. For some of the cases in Table 3.1, TEC- 6 was unable to hold the wall temperature at $\mathrm{X}=48.5 \mathrm{~cm}$ to $45^{\circ} \mathrm{C}$, so it ran at full power instead.

(iii) Two thermoelectric coolers (TEC-9 and TEC-10 of Fig. 3.1b), removing heat from an effective area that lies between $x=80 \mathrm{~cm}$ and $x=100 \mathrm{~cm}$, are maintained at a sufficient constant voltage (approximately constant heat absorption rate) to ensure that the fluid completely condenses within the test-section.

The above-described cooling approaches are used to define the condensing-surface thermal boundary conditions. For the data presented in this paper, we have defined two different cooling conditions. "Cooling-condition-1," termed "passive" cooling (PC), consists of using method (i) for all locations under the condensing plate (see Fig. 3.1). "Cooling-condition-2," termed "mixed" cooling (MC), consists of using methods (ii) and (iii) at the indicated locations over and above the continued use of the method (i) at all the other locations. Earlier data (not reported here, but reported in [31]) only used the "mixed" cooling-condition-2 approach. Also, similar steady cooling approaches were used in [30-31]. 


\subsubsection{Procedures}

\subsubsection{No-imposed fluctuation steady/quasi-steady flows}

The procedure is for achieving nearly steady (quasi-steady, with no deliberately imposed fluctuations) - also called "natural" - fully condensing flows, whose effective point of full condensation is within the test-section. In this context, "natural" is taken to mean without pulsator imposed fluctuations at the inlet, although ubiquitous miniscule perturbations on the flow caused by wall vibrations, vapor turbulence upstream of the condenser, etc. may still be present. Downstream of the exit (including the "visualization chamber" in Fig. 3.2), the flow loop is all-liquid up to the evaporator. This procedure involves:

(i) keeping the compressor and the pulsator off with the bypass valve $\left(V_{B P}\right.$ in Fig. 3.2) fully open,

(ii) fixing the evaporator bath temperature $T_{\text {bath }}$,

(iii) holding fixed the Coriolis mass flow meter $F_{c}$ (in Fig. 3.2) reading of the mass flow rate $\overline{\mathrm{M}}_{\text {in }}$ by a PID control of the evaporator internal heater,

(iv) steadying the condensing-surface temperature to a profile $\bar{T}_{w}(x)$ with the help of one of the cooling approaches described in section 3.3.3, and

(v) using the controllable displacement pump P, through a PID control, to hold the mean inlet pressure fixed at $\bar{p}_{\text {in }}=p_{\text {in }}^{*}$. This procedure allows the mean exit pressure $\bar{p}_{\text {exit }}$ to freely seek its no-imposed-fluctuation (abbreviated "N-IF" hereafter) steady value $\bar{p}_{\text {exit|N-IF }}$ and defines the no-imposed-fluctuation (N-IF) quasi-steady flow with its self-sought pressure difference $\overline{\Delta p}_{\mid \mathrm{N}-\mathrm{IF}}=\mathrm{p}_{\mathrm{in}}^{*}-\overline{\mathrm{p}}_{\mathrm{exit|NF}} \equiv \overline{\Delta \mathrm{p}}_{\mid \mathrm{Na} a}$. A more comprehensive experimental characterization of these natural quasi-steady flows has been reported in [40].

\subsubsection{Quasi-steady response to imposed fluctuations}

In this procedure, the mean mass flow rate $\overline{\mathrm{M}}_{\text {in }}$, mean inlet pressure $\overline{\mathrm{P}}_{\text {in }}$, and the steady cooling conditions remain the same as the ones obtained for the original no-fluctuation flow in section 3.3.4.1. The compressor continues to remain off with the bypass valve, 
$\mathrm{V}_{\mathrm{BP}}$, fully open. In addition, the bath temperature, $\mathrm{T}_{\text {bath }}$, surrounding the evaporator is also kept constant. The pulsator-supplied frequencies for the pressure fluctuations at the test-section inlet are predominantly the frequency (or the rpm) of the motor driving the pulsator and its harmonics. For Tables 3.1 and 3.2 in this chapter (also Tables $1-2$ in [1]), the harmonics are not separately characterized, and the fluctuational components of the reported pressures and heat-flux are assumed to be adequately characterized by their amplitudes at the pulsator driving frequency, $f_{P}$ (in the $0-29 \mathrm{~Hz}$ range), corresponding to the pulsator motor speed (RPM). This is the frequency corresponding to the highest physically significant peak in the FFT of $\mathrm{p}_{\text {in }}^{\prime}(\mathrm{t})$ (see Fig. 3.20). The amplitude $\mathrm{a}_{\mathrm{p} \text {-in }}$ of the pressure fluctuations associated with this frequency $f_{P}$ is increased by increasing the level of opening of the valve $V_{P}$ in Fig. 3.2. When valve $V_{P}$ is closed, this amplitude $a_{p-i n}$ is effectively zero.

The above approaches allow for impositions of different test-section inlet pressure fluctuation amplitudes, $a_{p-i n}$, and fluctuation frequencies, $f_{p}$. These impositions (for the same quasi-steady inlet pressure) are shown here to lead to different quasi-steady flows with different values of quasi-steady local heat-flux (in the annular regime) and different mean lengths, $\bar{X}_{A}$, of the annular regime (see Fig. 3.4). For these new quasi-steady imposed-fluctuation (abbreviated "IF" hereafter) cases, mean values and amplitudes of pressure-differences $\left(\overline{\Delta \mathrm{p}}_{40 \mathrm{~cm}}\right.$ and $\left.\overline{\Delta \mathrm{p}}_{90 \mathrm{~cm}}\right)$, local heat-flux $\left(\overline{\mathrm{q}}_{40 \mathrm{~cm}}\right.$ at HFX-1 in Fig. 3.1b), test-section thermocouple readings (reported as $\bar{T}_{40 \mathrm{~cm}}$ and $\bar{T}_{w \mid R e f}$ ), length $x_{A}$ of the annular regime (see Figs. 3.1a and 3.4), etc., are recorded (see Table 3.1). 



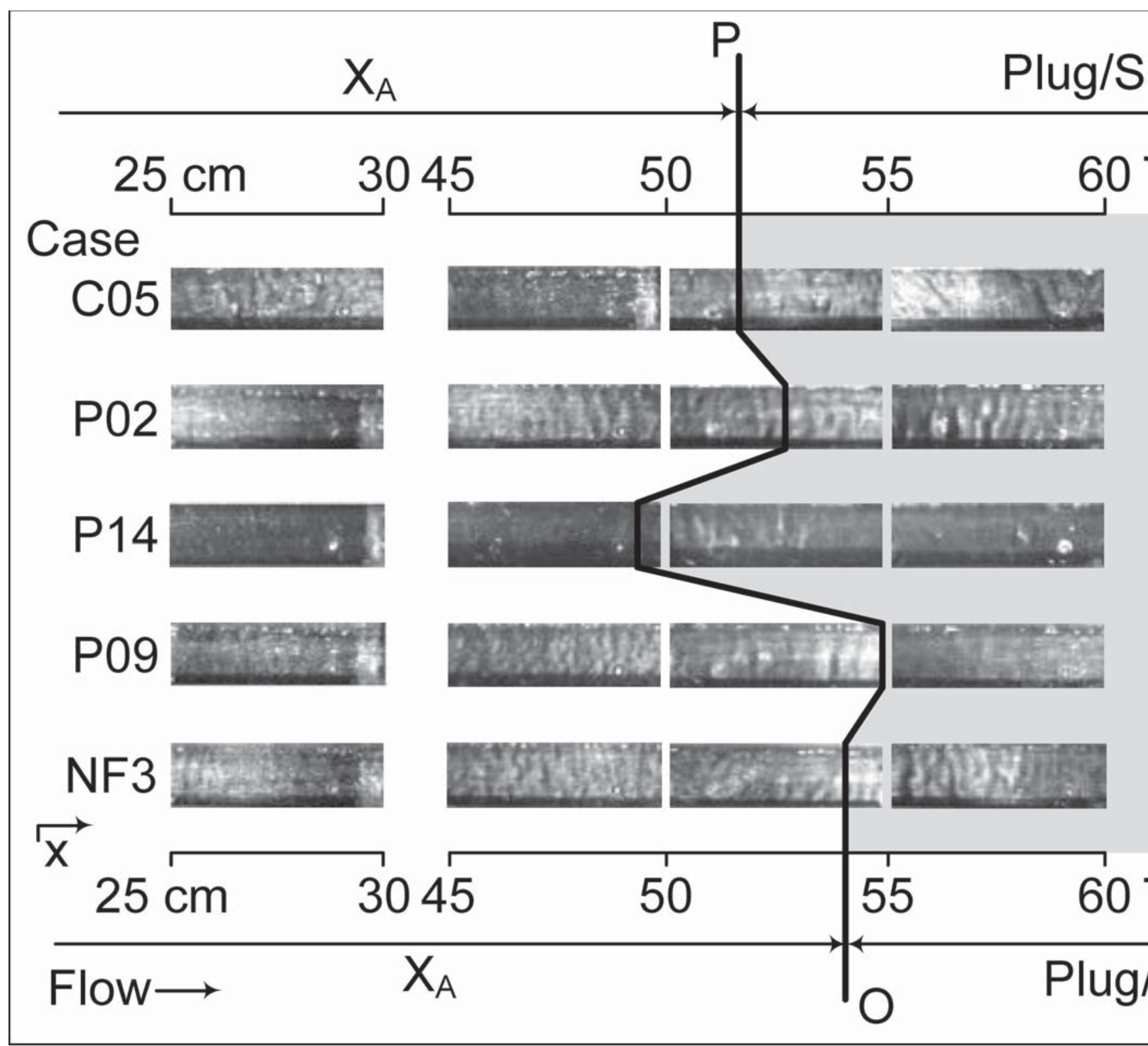

Figure 3.4. Sample flow visualization photographs for select cases in Table 3.1. The length of the annular flow reg corresponding strip picture. The non-annular flow regime sections also have background shading in between the $p$ 



\subsection{Results}

This section represents a significant expansion on the results presented in the original publication of this paper [1]. New figures have been added, and discussions have been expanded and updated to reflect new understanding presented in chapter 2. Some of the content has also been edited for clarification and readability.

\subsubsection{No-imposed fluctuation condensing flow results}

As depicted in Figs. $3.5-3.6$, over the time intervals $t_{1} \leq t \leq t_{1}^{*}$, the procedure described in section 3.3.4.1 is effective in achieving a unique no-imposed fluctuation (N-IF) quasi-steady natural flow for $\overline{\mathrm{M}}_{\text {in }} \approx 1.20 \mathrm{~g} / \mathrm{s}$, quasi-steady cooling-condition-1 as specified in section 3.3.3 (which corresponds to nearly steady N-IF wall temperature distributions $\bar{T}_{w}(x)$ shown in Figs. 3.7 and 3.9), and approximately quasi-steady inlet pressure set at $\mathrm{p}_{\mathrm{in}}^{*}=142 \mathrm{kPa}$. For achieving these flows, the compressor and pulsator were off. The resulting flow has an effective point of full condensation near the test-section exit and has a flow morphology that changes (with distance from the test-section inlet) from wavy annular to slug/plug to bubbly to all-liquid regimes (see Figs. 3.1a and 3.4).

For case NF3 in Table 3.1, we see that the annular regime differential pressure transducer (DPT-1 in Fig. 3.1a) records the pressure drop (denoted as $\overline{\Delta p}_{40 \mathrm{~cm}}$ ) with the value of approximately $131 \mathrm{~Pa}$, which is quite small relative to the mean inlet pressure of $142 \mathrm{kPa}$. Also, the heat-flux in the annular region at the HFX-1 location (see Fig. 3.1b) is $\overline{\mathrm{q}_{40 \mathrm{~cm}}^{\prime \prime}} \approx 0.51 \mathrm{~W} / \mathrm{cm}^{2}$ as shown in Fig. 3.6 and Table 3.1. The uniqueness and repeatability of these realizations have been shown elsewhere (see [30] and [40]) by a slightly different procedure. 


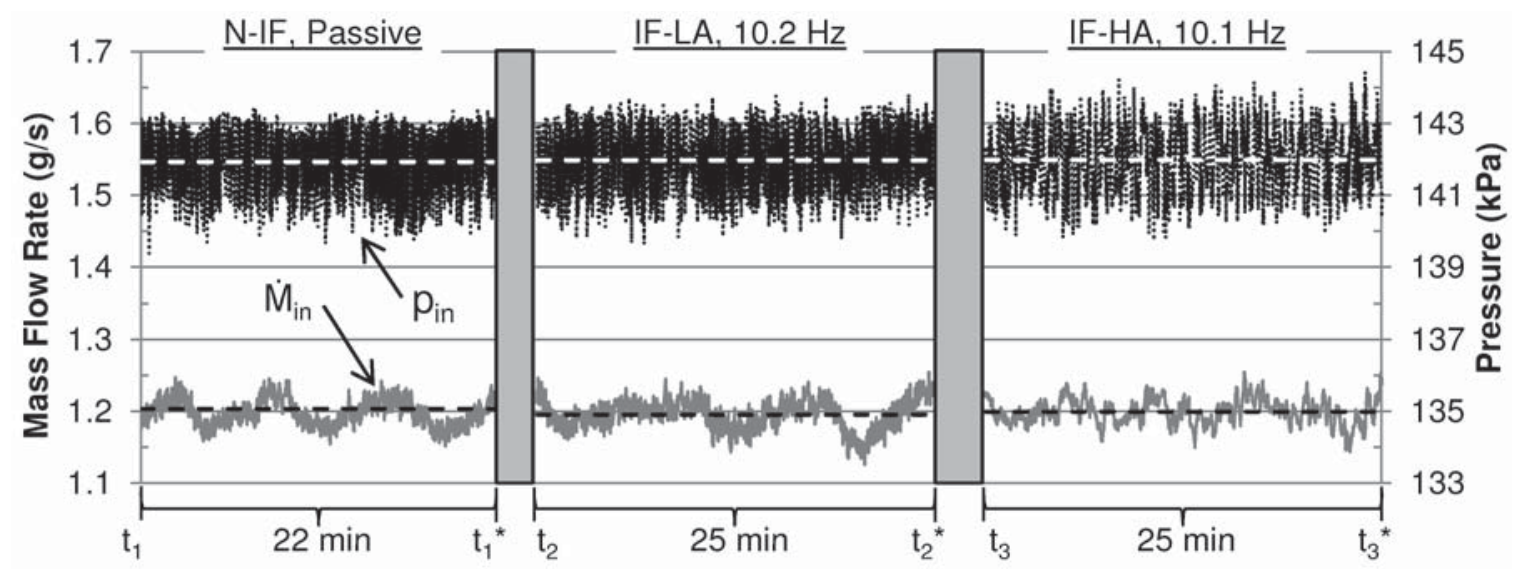

Figure 3.5. Inlet mass flow rate and pressure measured at low DAQ rate for "passive" cooling condition cases NF3, P07, and P02 in Table 3.1. Dashed lines represent long-term time-averages of the data. These cases are termed no-imposed fluctuations (N-IF), low amplitude imposed fluctuations (IF-LA), and high amplitude imposed fluctuations (IF-HA) respectively.

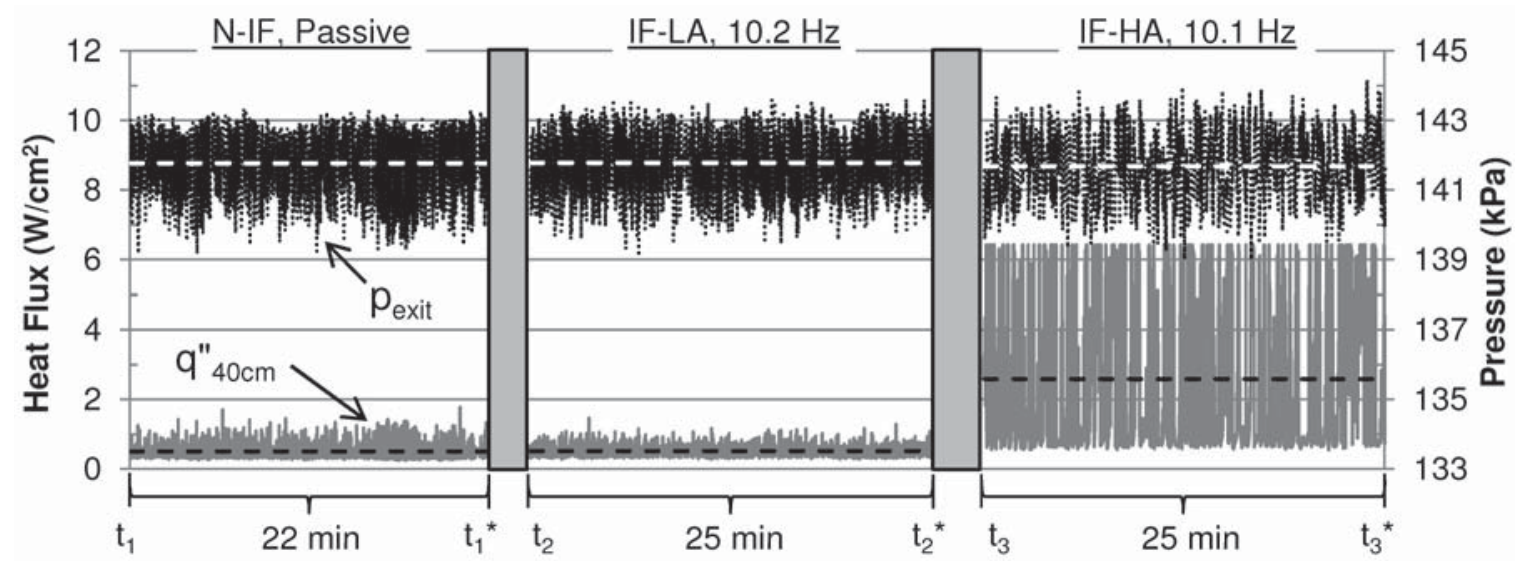

Figure 3.6. Heat-flux (at $x=40 \mathrm{~cm}$ ) and exit pressure measured at low DAQ rate for "passive" cooling condition cases NF3 (N-IF), P07 (IF-LA), and P02 (IF-HA) in Table 3.1. Dashed lines represent long-term time-averages of the data. Note the enhancement in the mean heat-flux (black dashed line) for the high amplitude imposed fluctuation (IF-HA) case. The mean inlet and exit pressure values are not precise enough to yield an accurate differential pressure (when exit pressure values are subtracted from inlet pressure values) due to calibration uncertainty. Note that the heat-flux $q_{40 \mathrm{~cm}}^{\prime \prime}$ for IF-HA case P02 was outside of the measurement range for the low data acquisition rate data reported here; however, it was within the measurement range for the dynamic data reported in Fig. 3.13. Average values of $q_{40 \mathrm{~cm}}^{\prime \prime}\left(\overline{q_{40 \mathrm{~cm}}^{\prime \prime}}\right)$ used to generate the black dashed line and reported in Table 3.1 for case P02 were obtained using the dynamic data (which had a higher measurement range), so their values are trustworthy. 


\subsubsection{Quasi-steady condensing flows with imposed inlet-pressure fluctuations}

In the presence of a steady pulsator driving frequency, following the procedure described in section 3.3.4.2, as the compressor remained off and the pulsator diaphragm frequency was brought from zero to different steady values $(3.9 \mathrm{~Hz}, 10.2 \mathrm{~Hz}, 10.5 \mathrm{~Hz}$, and 16.1 Hz), different quasi-steady imposed-fluctuation cases were achieved. For these quasi-steady cases, the impositions of different time-varying inlet pressures are represented as:

$\mathrm{p}_{\text {in }}(\mathrm{t}) \equiv \overline{\mathrm{p}}_{\mathrm{in}} \mathrm{l}_{\mathrm{IF}}+\mathrm{p}_{\mathrm{in}}^{\prime}(\mathrm{t})$,

where

$p_{i n}^{\prime}(t) \cong\left[a_{p-i n} \cdot \sin \left(2 \cdot \pi \cdot f_{p} \cdot t\right)\right]+$ other terms.

The value of the amplitude $a_{p-i n}$ in Eq. (3.10) is the amplitude associated with the predominant pulsator frequency ' $f_{p}$ ' in the Fast Fourier Transform (FFT) spectrum obtained for the dynamic inlet pressure transducer signal $p_{i n}(t)$. Analogous to Eqs. (3.9) - (3.10), for any other dynamic variable $X(t)$ associated with these quasi-steady flows, the notations used are:

$\left.X(t) \equiv X_{i n}\right|_{I F}+X^{\prime}(t)$,

where

$X^{\prime}(t) \cong\left[a_{X} \cdot \sin \left(2 \cdot \pi \cdot f_{P} \cdot t+\varphi_{X}\right)\right]+$ other terms.

The experimentally measured values of $\dot{M}_{i n}(t)$ and $p_{i n}(t)$ obtained at the lower DAQ rate of $0.91 \mathrm{~Hz}$ are shown in Fig. 3.5, the corresponding measured values of the $q_{40 \mathrm{~cm}}^{\prime \prime}(t)$ and $p_{\text {exit }}(t)$ are shown in Fig. 3.6, and the corresponding measured temperature values of $\bar{T}_{w}(x)$ are shown in Fig. 3.7. In Figs. 3.5 - 3.6, results for a representative lower amplitude imposed fluctuation case (termed IF-LA) are shown over $t_{2} \leq t \leq t_{2}^{*}$, and a representative higher amplitude fluctuation case (termed IF-HA) is shown over $t_{3} \leq t \leq t_{3}^{*}$. Passive cooling condition is used, and the pulsation frequency is approximately $10.2 \mathrm{~Hz}$. 


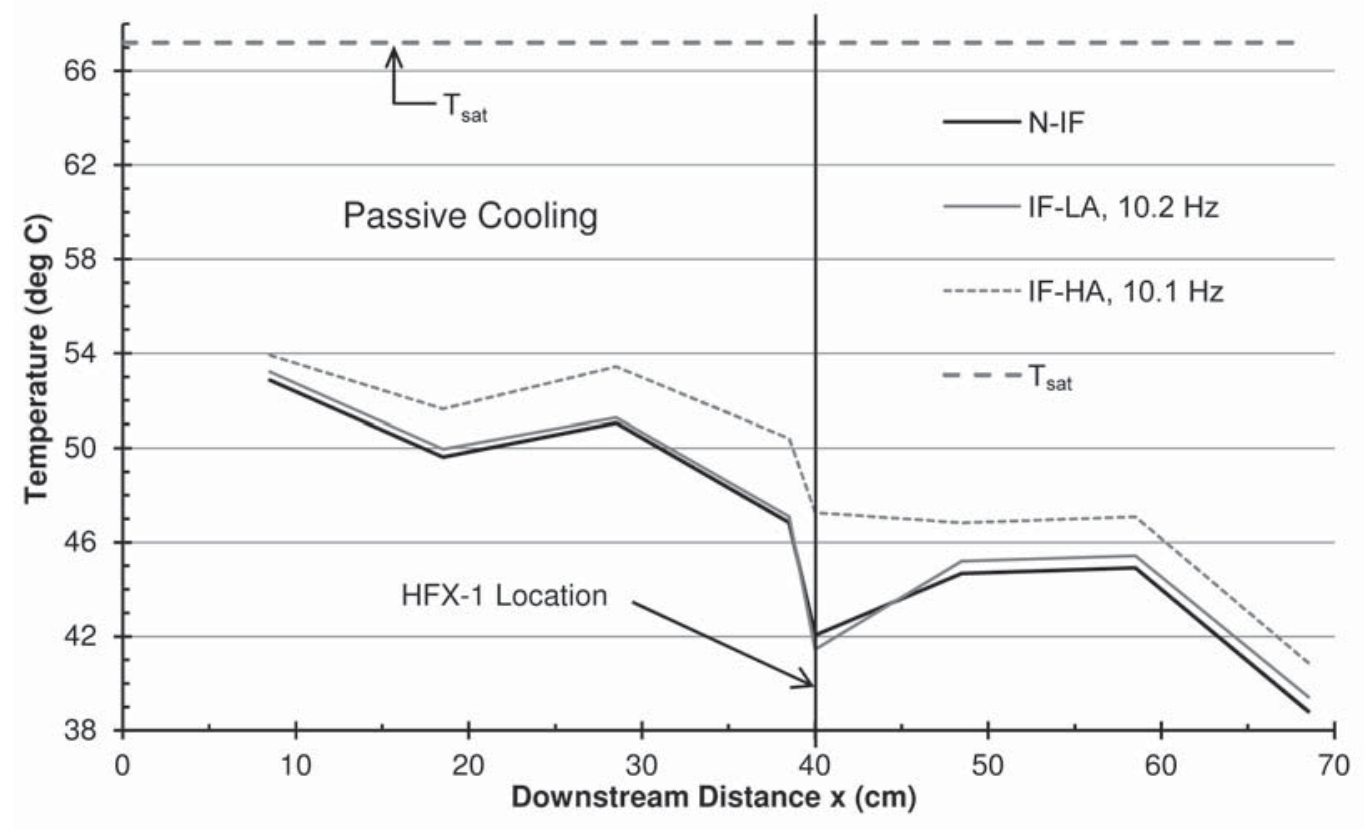

Figure 3.7. Quasi-steady wall temperature profiles under "passive" cooling conditions, with coolant (water) inlet temperature and flow rate maintained near $15^{\circ} \mathrm{C}$ and $7.9 \mathrm{lpm}$, respectively. The N-IF, IF-LA, and IF-HA cases correspond to cases NF3, P07, and P02 from Table 3.1.

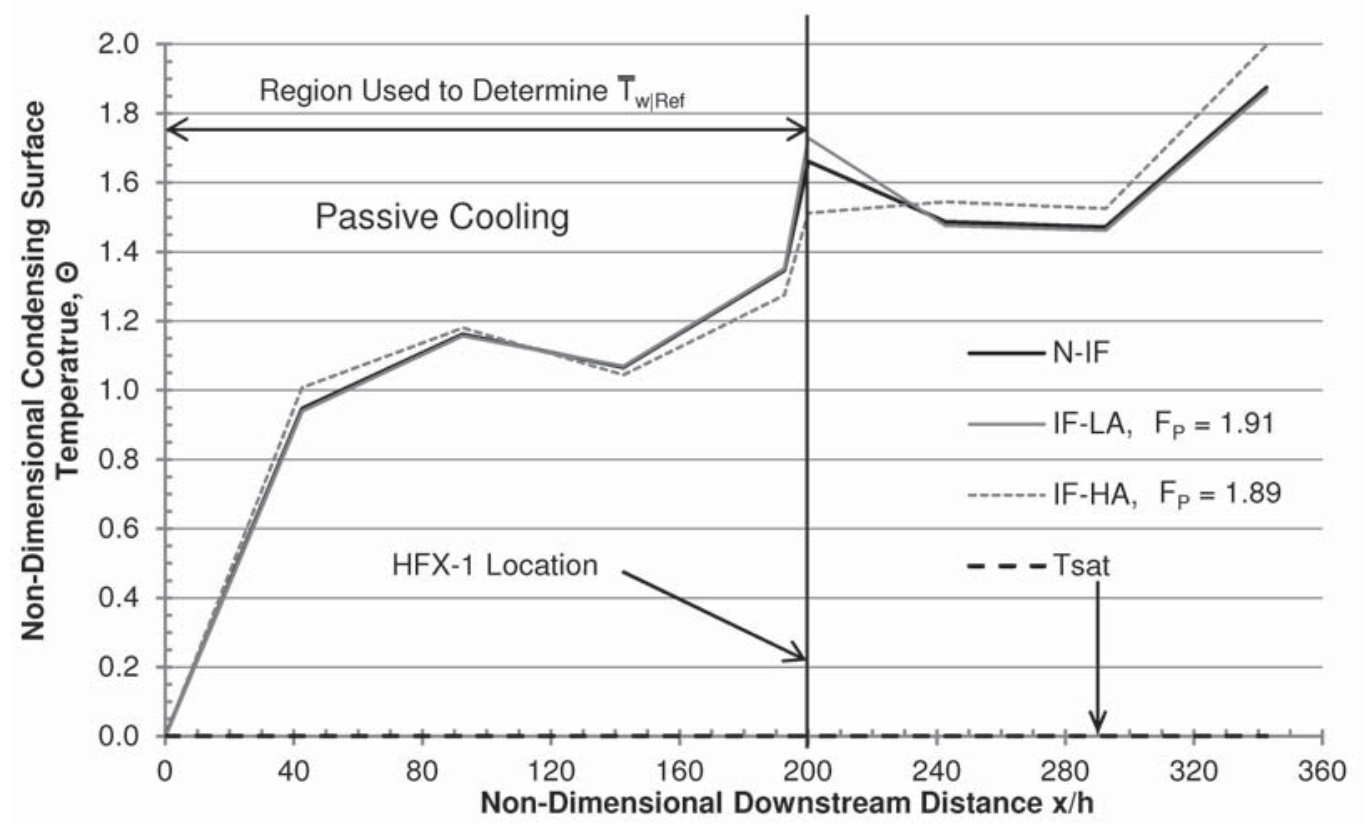

Figure 3.8. Non-dimensional wall temperature distribution chart under "passive" cooling conditions. This is the non-dimensional representation of Fig. 3.7 for cases NF3, P07, and P02 from Tables $3.1-3.2$. The average wall temperature $\bar{T}_{w / R e f}$ is represented by $\Theta=1$. 
The non-dimensional condensing-surface temperature profile in Fig. 3.8 approximately satisfies the Eq. (3.4) requirement that all temperature profiles of Fig. 3.7 lead to an approximately "single" curve (representing the mean of the depicted curves). This curve in Fig. 3.8 defines the "cooling-condition-1."

Due to the control strategy described in section 3.3, the mean inlet mass flow rate and inlet pressure were maintained at their N-IF quasi-steady values throughout the imposition of pulsations of low and high amplitude. This fact is also evident in Fig. 3.5. As a result of the higher heat-flux values associated with IF-HA cases and the nature of the "passive" steady cooling condition in section 3.3.3 (which allows surface temperature variations), following thermal transients associated with the thick condensing plate, different quasi-steady wall temperature distributions $\left.\bar{T}_{w}(x)\right|_{I F-H A} \neq\left.\left.\bar{T}_{w}(x)\right|_{I F-L A} \approx \bar{T}_{w}(x)\right|_{N-I F}$ are achieved, and this is shown in Fig. 3.7.

In contrast, for the "mixed" cooling condition (see section 3.3.3) which fixes condensing surface temperatures at two locations, the variations in the wall temperature are significantly reduced as shown in Fig. 3.9. Also, analogous to Fig. 3.8, an approximate single "mixed" cooling condition (defined as Cooling Condition 2 in section 3.3.3) is again defined by an approximate "single" curve by the non-dimensional wall temperature profiles (see Eq. 3.4) corresponding to these cases, and it is represented by the temperature profile in Fig. 3.10. 


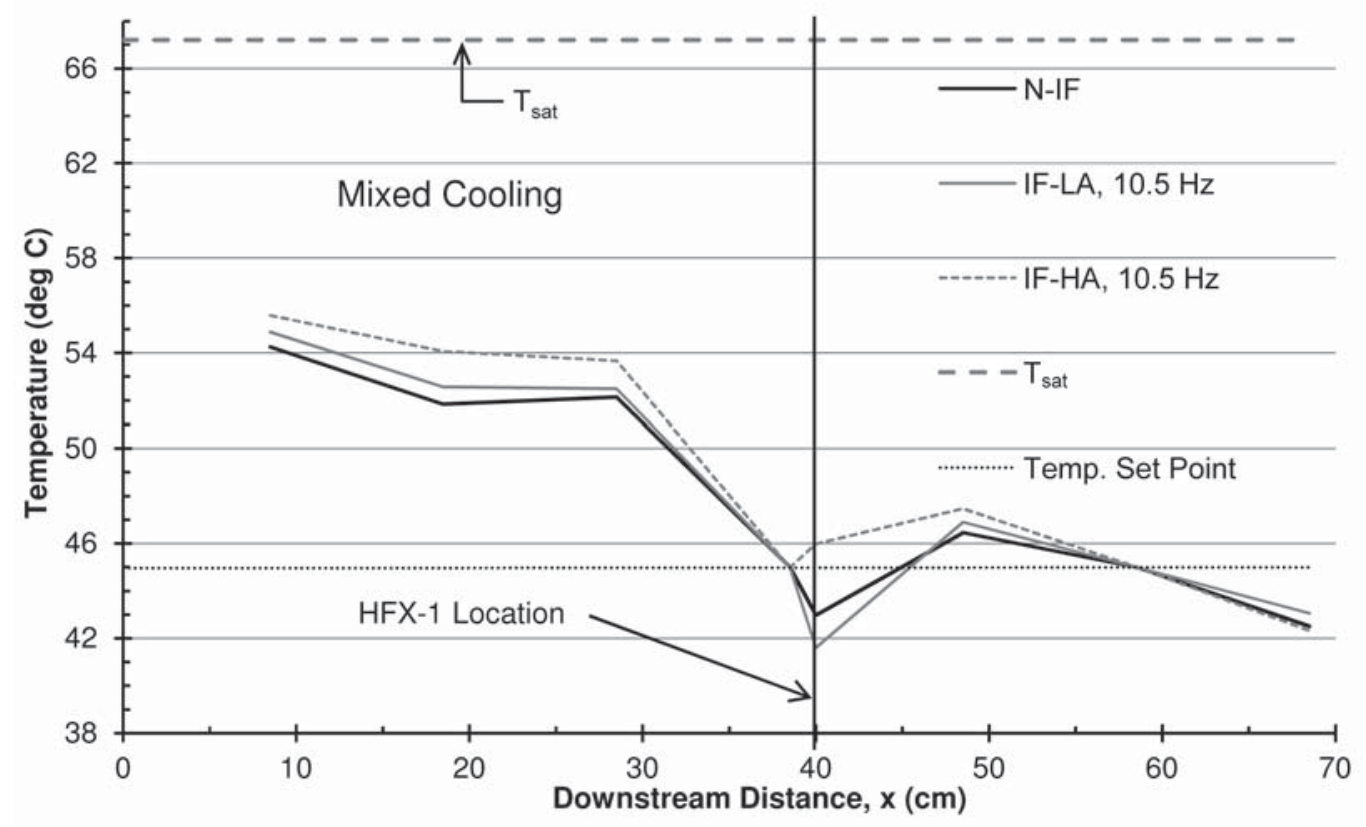

Figure 3.9. Quasi-steady wall temperature profiles under the "mixed" cooling conditions, with temperatures approximately held fixed at $38.5 \mathrm{~cm}$ and $58.5 \mathrm{~cm}$ downstream of the inlet. The N-IF, IF-LA, and IF-HA cases correspond to cases NF1, C15, and C05 from Table 3.1.

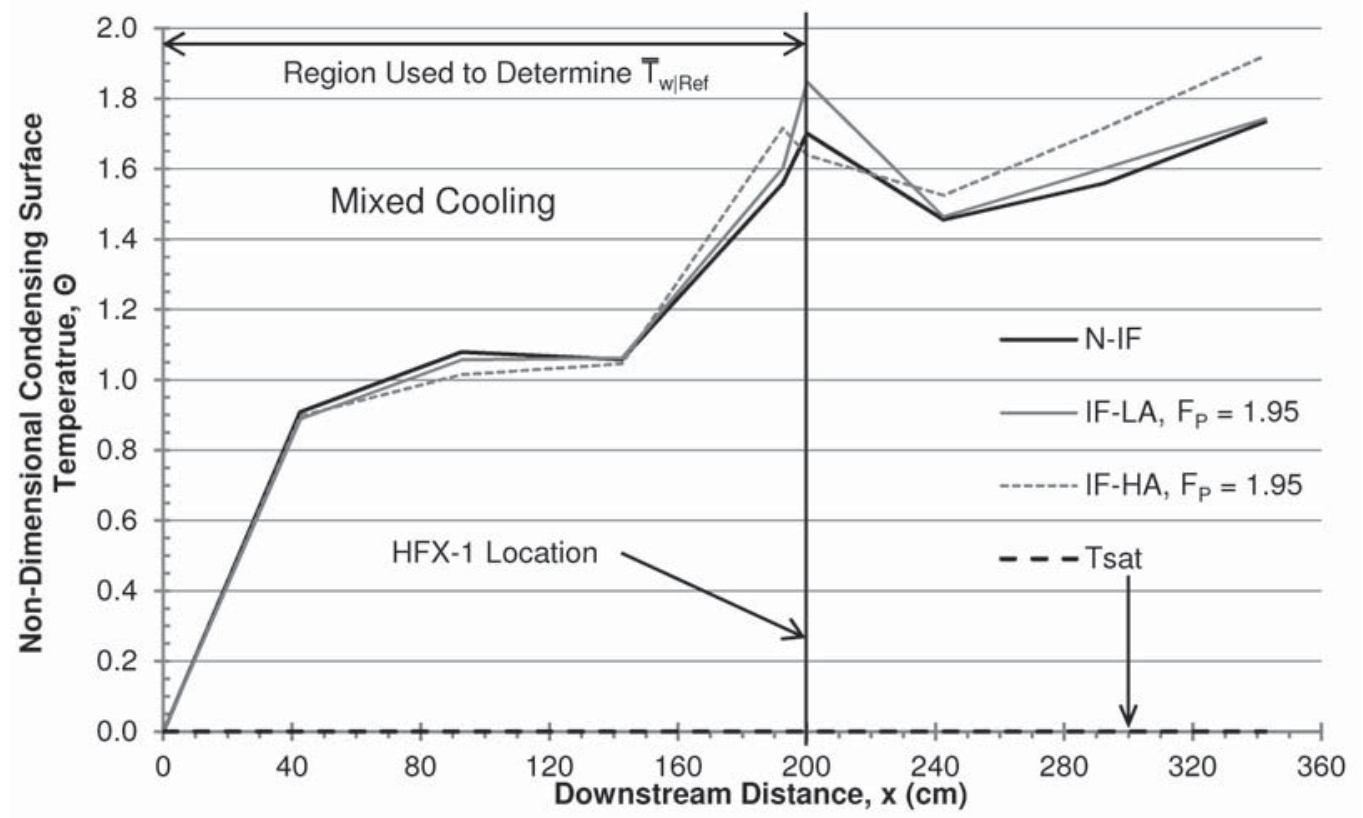

Figure 3.10. Non-dimensional wall temperature distribution chart under the "mixed" cooling conditions. This is the non-dimensional representation of Fig. 3.9 for cases NF1, C15, and C05 from Tables $3.1-3.2$. The average wall temperature $\bar{T}_{w / R e f}$ is represented by $\Theta=1$. 
The mean exit liquid pressure shifted downward slightly for the passive cooling (PC) condition IF-HA case in Fig. 3.6, but no such shift was significant for any of the mixed cooling (MC) condition cases of Table 3.1. However, for the cases considered in Figs. 3.5 - 3.6, the main effect of the high amplitude pressure fluctuations on the annular flow was an approximately $380 \%$ increase in the mean heat-flux at the $40 \mathrm{~cm}$ location, $\overline{\mathrm{q}_{40 \mathrm{~cm}}^{\prime \prime}}$, compared with the corresponding N-IF case NF3. Similar enhancement $(320-460 \%)$ in $\overline{q_{40 \mathrm{~cm}}^{\prime \prime}}$ was observed for IF-HA cases at all of the frequencies investigated under both cooling conditions (see $\overline{\mathrm{q}_{40 \mathrm{~cm}}}$ increase in Table 3.2). The underlying reasons for this enhancement, to be discussed later, are dynamic, and this is clear from the dynamic data (acquired at $2000 \mathrm{~Hz}$ ) for inlet pressure $\mathrm{p}_{\mathrm{in}}(\mathrm{t})$ shown in Fig. 3.11, pressure-difference $\Delta p_{40 \mathrm{~cm}}(t)$ shown in Fig. 3.12 and heat-flux $q_{40 \mathrm{~cm}}^{\prime \prime}(t)$ shown in Fig. 3.13 for the passive cooling condition. Similar dynamic behavior for these measured parameters occurred under the mixed cooling condition, but their time-histories are not reported here for brevity. Sample visualization pictures for select cases in Fig. 3.4 show the effects of imposed fluctuations on the flow morphology and on the length of the annular flow regime.

Over $t_{2} \leq t \leq t_{2}^{*}$ (case P07 in Tables $3.1-3.2$ ) and $t_{3} \leq t \leq t_{3}^{*}$ (case P02 in the Tables), the time characteristics of the inlet pressure, inlet mass flow rate, exit pressure, and heat-flux in Figs. 3.5 - 3.6 do not represent their actual fluctuations (see Figs. $3.11-3.13$ ) at the dominant frequency $f_{P} \approx 10 \mathrm{~Hz}$ present in these signals because of the significantly lower DAQ rate used (approximately $0.91 \mathrm{~Hz}$ ). Only variations over longer periods of time (on the order of minutes) are captured in Figs. 3.5 - 3.6.

Note that the values of $\overline{\mathrm{q}_{40 \mathrm{~cm}}}$ for the high amplitude cases as reported in Table 3.1 and elsewhere were obtained by averaging dynamic data of the type shown in Fig. 3.13 for individual dynamic data sets and then averaging these averages for multiple dynamic data sets taken over sufficiently long periods of time. In other words, the clipped low DAQ rate data of the type shown in Fig. 3.6 for $t_{3} \leq t \leq t_{3}^{*}$ were not used in obtaining any reported $\overline{\mathrm{q}_{40 \mathrm{~cm}}^{\prime \prime}}$ values. 
A representative data-matrix, in terms of the amplitude $\mathrm{a}_{\text {p-in }}$ (defined in Eqs. 3.9 - 3.10) and frequency $f_{p}$ of the inlet pressure fluctuation impositions, is shown in Fig. 3.14. Figure 3.15 reveals the fact that, for a given frequency $f_{p}$, the heat-flux enhancement increases significantly with amplitude for cases whose amplitudes are above the Transition Region- 1 - with diminishing returns eventually setting in at amplitudes above the Transition Region-2.

The dynamic pressure-difference data from the specially designed orifice plate meter in Fig. 3.2 is processed, with the help of the new method described in [41], to yield the dynamic mass flow rate signal $\dot{\mathrm{M}}_{\mathrm{OM}}(\mathrm{t})$ at the exit of the orifice meter. Applying ad-hoc corrections accounting for transient compressibility effects as a first order estimate ([41]), and knowing that the principal harmonic effect dominates as far as inlet mass flow $\dot{M}_{\text {in }}(t)$ is concerned, it suffices to show a representative (for case P02 in Table 3.1) $\dot{M}_{\text {in }}(t) \cong \dot{M}_{\mathrm{OM}}(t) \cdot C$ in Fig. 3.3, where 'C' accounts for compressibility effects ([41]) between the orifice-meter exit and the test-section inlet.

As discussed later, the basic result, that the principal (fundamental) amplitude $\mathrm{a}_{\mathrm{M}-\text { in }}$ of inlet mass flow rate fluctuations $\dot{\mathrm{M}}_{\text {in }}(\mathrm{t})$ is large compared to its mean value $\overline{\mathrm{M}}_{\text {in }}$ and is greater than all harmonics, remains true for all high amplitude imposed fluctuation cases. 


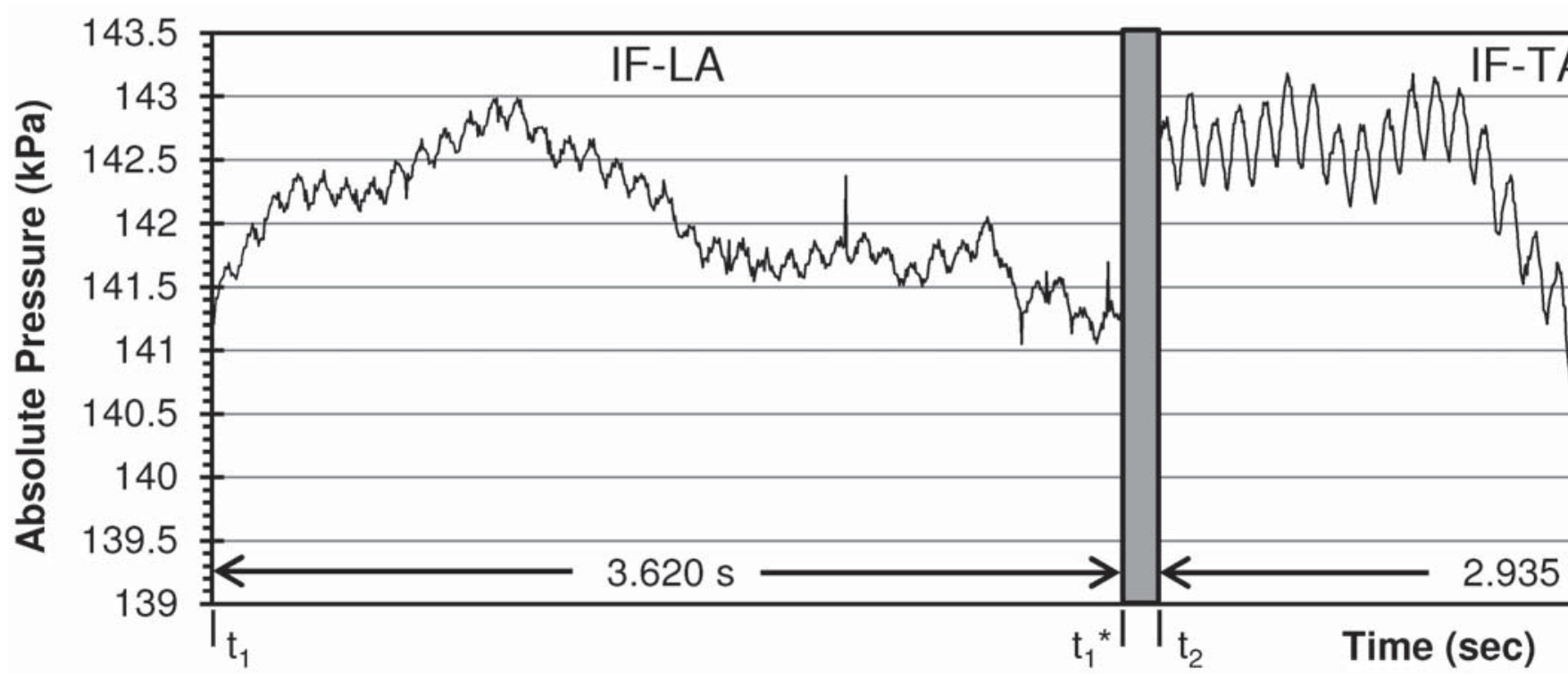

Figure 3.11. Dynamic data of the inlet vapor pressure $p_{i n}(t)$ for low amplitude (IF-LA), transition amplitude (IF-TA), cases P07, P05, and P02, respectively, in Table 3.1.

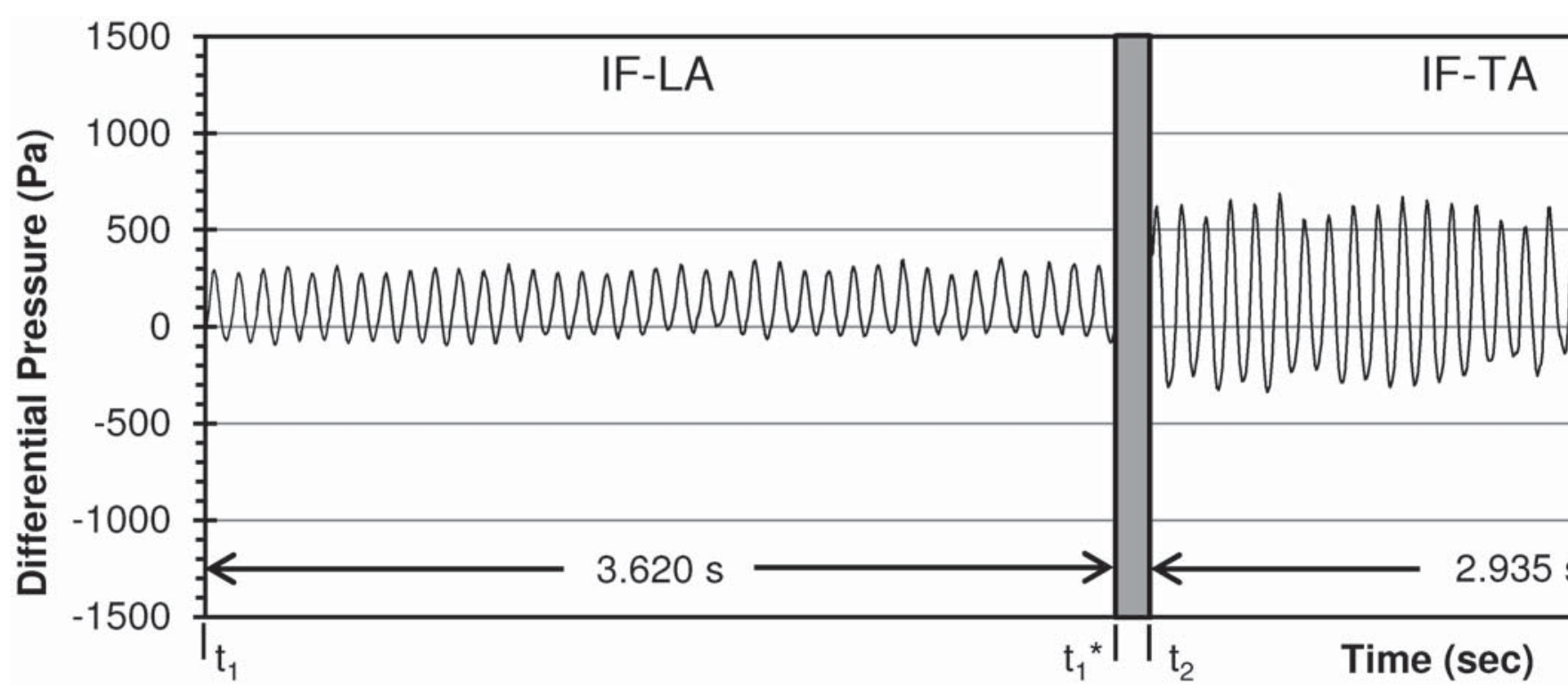

Figure 3.12. Dynamic data of the pressure-drop $\Delta p_{40 \mathrm{~cm}}(t)$ for low amplitude (IF-LA), transition amplitude (IF-TA), a cases P07, P05, and P02, respectively, in Table 3.1. 



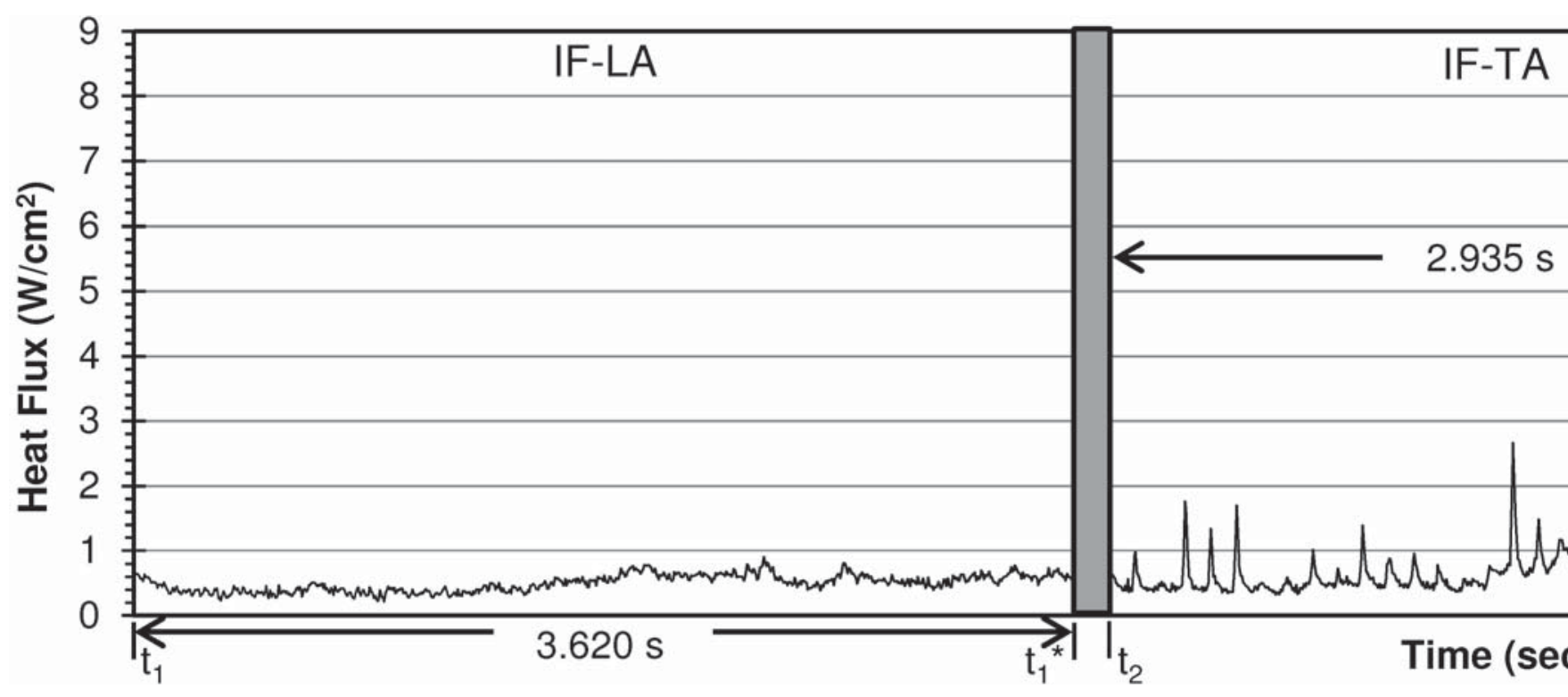

Figure 3.13. Dynamic data of annular flow regime heat-flux $q_{40 \mathrm{~cm}}^{\prime \prime}(t)$ for low amplitude (IF-LA), transition amplitude condition cases P07, P05, and P02, respectively, in Table 3.1. 



\subsubsection{Experimental observation of heat-flux enhancement and changes to the flow due to imposed fluctuations}

\subsubsection{Annular regime}

Figures $3.5-3.13$ show that the effects of fluctuations in the vapor flow does (for IF-HA cases) or does not (for IF-LA cases) significantly change the quasi-steady flow variables associated with changes in time-averaged heat-flux (or mean condensate thickness), flow morphology, or condensate motion relative to the natural (N-IF) case over $\mathrm{t}_{1} \leq \mathrm{t} \leq \mathrm{t}_{1}^{*}$. The variables affected include mean heat-flux $\overline{\mathrm{q}_{40 \mathrm{~cm}}}$ in Figs. 3.6 and 3.13, time-averaged condensing-surface temperatures $\bar{T}_{w}(x)$ in Figs. 3.7 and 3.9, flow morphology in Fig. 3.4, etc.

For the $f_{P}=10.2 \mathrm{~Hz}$ case, as the valve $V_{P}$ in Fig. 3.2 is gradually opened to increase the pulsation amplitude associated with point $P_{1}$ to that associated with point $P_{2}$ in Fig. 3.14, a transition point $T_{1}$ or a Transition Region-1 (see Figs. 3.14-3.15) is crossed. A similar transition point is crossed for any other frequency (e.g., $T_{2}$ is a representative transition point in Fig. 3.14 for $\left.f_{p}=16.2 \mathrm{~Hz}\right)$, and these transition cases $\left(T_{1}, T_{2}\right.$, etc. $)$ are denoted "IF-TA" in Fig. 3.14 and Tables 3.1 - 3.2. 


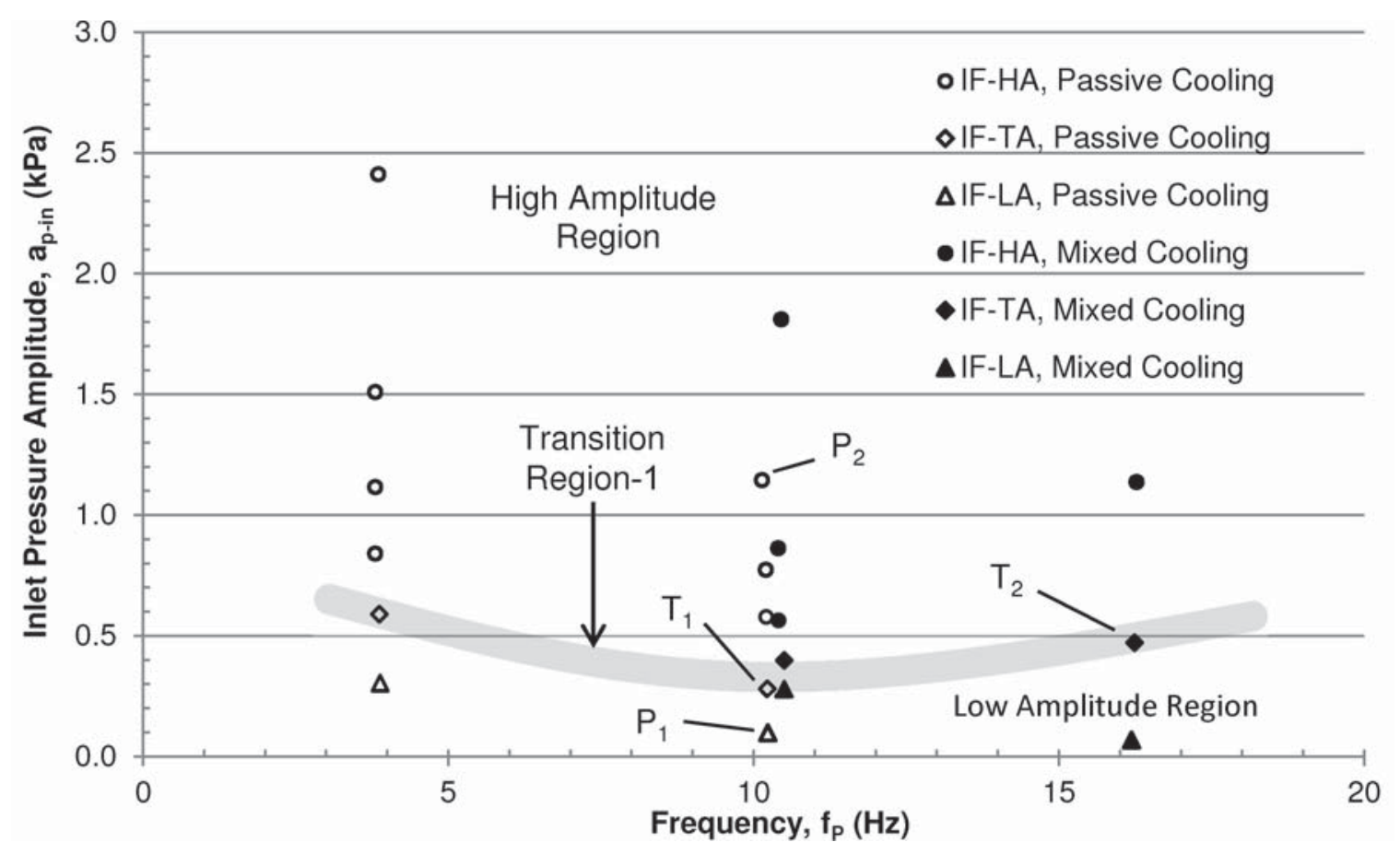

Figure 3.14. The amplitude-frequency data-matrix map for the imposed inlet pressure fluctuation cases in Table 3.1.

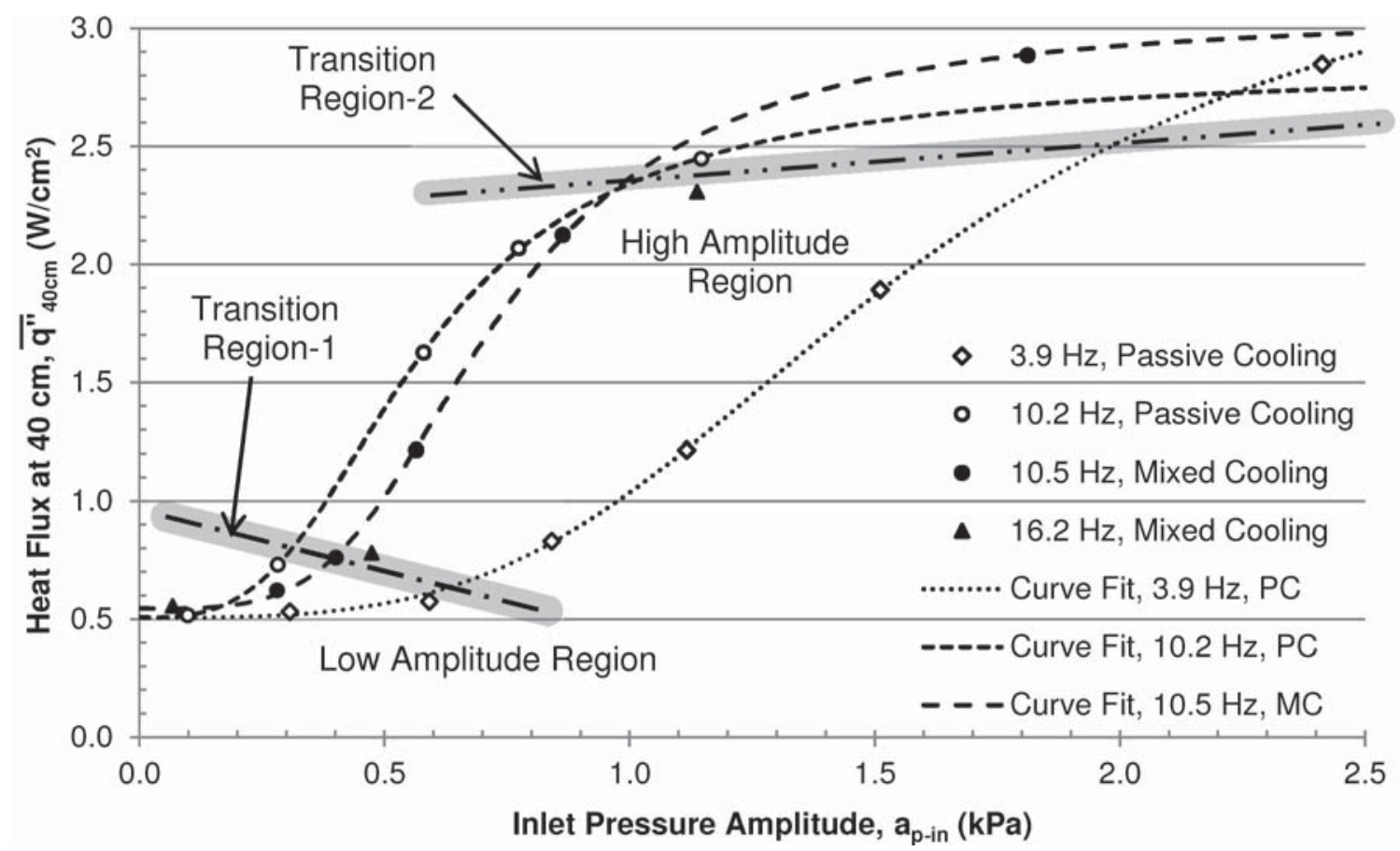

Figure 3.15. Heat-flux enhancement vs. $p_{i n}$ amplitude chart for different pulsator frequencies. The behavior is similar for two different condensing surface cooling conditions (Passive Cooling, PC, and Mixed Cooling, MC). 
The slope of the heat flux $\overline{q_{40 \mathrm{~cm}}^{\prime \prime}}$ versus inlet pressure fluctuation amplitude curve in Fig. 3.15 significantly increases as Transition Region-1 is crossed from low-amplitude to high-amplitude. From Fig. 3.13 it is also clear that the transition value of inlet pressure pulsations' amplitude corresponds to a case where the time-varying nature of heat-flux shows a pattern that is intermediate between the low amplitude and high amplitude pulsation cases. This intermediate feature, over $t_{2} \leq t \leq t_{2}^{*}$ in Fig. 3.13, exhibits occasional appearances and disappearances of the $10.2 \mathrm{~Hz}$ frequency peaks.

The representative mean value, $\overline{\Delta \mathrm{p}}_{40 \mathrm{~cm}}$, and real-time variations, $\Delta \mathrm{p}_{40 \mathrm{~cm}}(\mathrm{t})$, of the annular regime pressure drop are respectively shown in Table 3.1 and Fig. 3.12 for IF-LA, IF-TA, and IF-HA cases (P07, P05, and P02 respectively). The slight increase in mean values of $\overline{\Delta \mathrm{p}}_{40 \mathrm{~cm}}$ as one goes from IF-LA to IF-HA cases in Table 3.1 may provide an increase in the mean values of effective interfacial shear over $0 \leq x \leq 40 \mathrm{~cm}$, but it is not significant enough to cause the dramatic decrease in the mean value $\bar{\delta}(x)$ of the instantaneous condensate thickness profile $\delta(x, t)$, observed at the $x=40 \mathrm{~cm}$ location (determined after the publication of [1]). Instead, other factors (e.g. solid-liquid-vapor interaction in the interfacial wave troughs and wave-induced interface texturing) described in detail in sections 2.3.5 and 3.4.5 are now thought to be the primary causes of the pulsation-induced reduction in the time-averaged film thickness profile $\bar{\delta}(\mathrm{x})$. The time-varying nature of the instantaneous film thickness profiles $\delta_{40 \mathrm{~cm}}(t)$ for these three cases can be inferred (see Fig. 3.16) because the heat transfer in the thin annular liquid film is heavily dominated by conduction across the film (see [36 - 38]). Therefore, heat-flux may be related to film thickness by the following relationship:

$$
\mathrm{q}_{40 \mathrm{~cm}}^{\prime \prime}(\mathrm{t}) \cong \mathrm{k}_{\mathrm{L}} \cdot\left(\mathrm{T}_{\text {sat }}-\mathrm{T}_{40 \mathrm{~cm}}(\mathrm{t})\right) / \delta_{40 \mathrm{~cm}}(\mathrm{t})
$$

In Eq. (3.13), both $\mathrm{q}_{40 \mathrm{~cm}}^{\prime \prime}(\mathrm{t})$ (see Fig. 3.13) and $\mathrm{T}_{40 \mathrm{~cm}}(\mathrm{t}) \cong \bar{T}_{40 \mathrm{~cm}}$ (see Table 3.1) are directly measured by the heat-flux meter and its embedded thermocouple. The value of liquid thermal conductivity, $k_{L}$, is taken at the average of $T_{\text {sat }}$ and the measured $\bar{T}_{40 \mathrm{~cm}}$. 


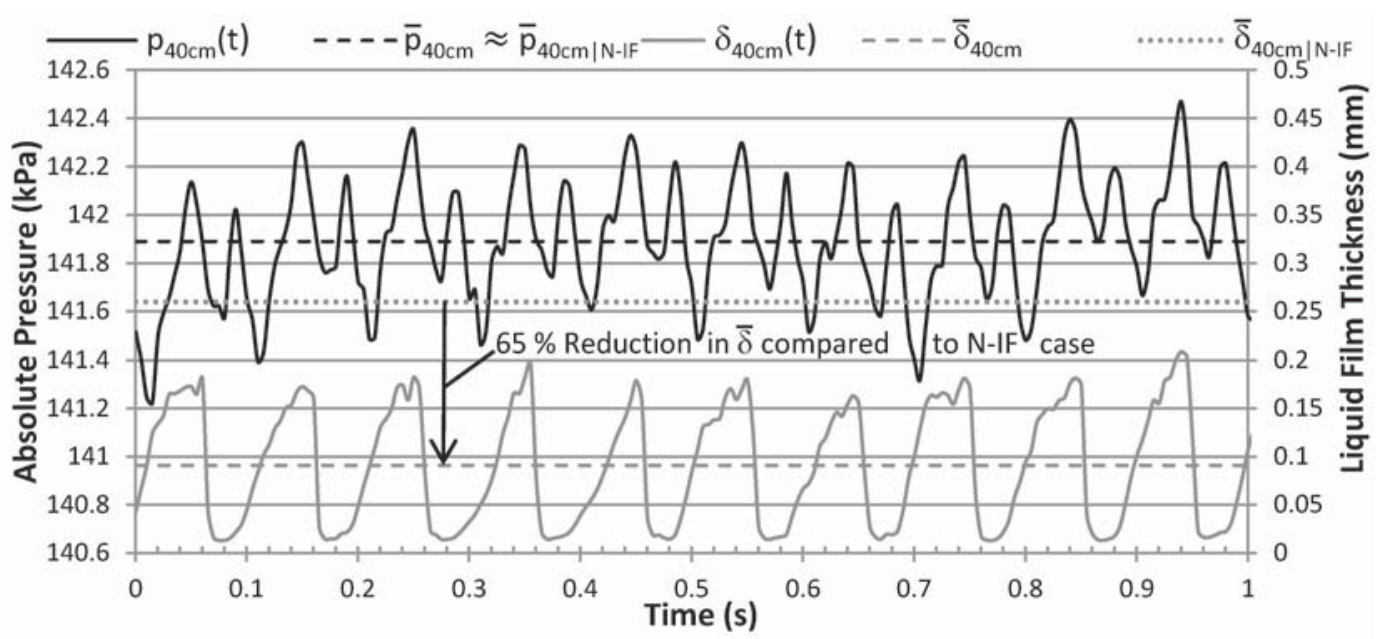

Figure 3.16. Dynamic calculated data for film thickness (see Eqs. $3.6-3.13$ ) and pressure $p_{40 \mathrm{~cm}}(t) \cong p_{\text {in }}(t)-\Delta p_{40 \mathrm{~cm}}(t)$. These are representative responses for the IF-HA case P02 in Table 3.1.

Although the transition points define a narrow band within the grey curve in Fig. 3.14, for present purposes it suffices to model it as a single transition amplitude curve $a_{p-i n}^{c r}(f)$. The Transition Region-1 in Fig. 3.14 partitions the data-matrix for the imposed inlet pressure pulsations into a subcritical zone below this transition curve and a supercritical zone above this transition curve. As an example, for the low amplitude IF-LA case associated with point $\mathrm{P}_{1}$ in the subcritical zone of Fig. 3.14, one has $\overline{\mathrm{q}_{40 \mathrm{~cm}}^{\prime \prime}} \approx 0.52 \mathrm{~W} / \mathrm{cm}^{2}$, and for the high amplitude IF-HA case associated with point $P_{2}$ in the supercritical zone of Fig. 3.14, one has $\overline{\mathrm{q}_{40 \mathrm{~cm}}^{\prime \prime}} \approx 2.45 \mathrm{~W} / \mathrm{cm}^{2}$ (> $370 \%$ increase). Utilizing the notations introduced in Eqs. (3.9) - (3.12) and the notation $\mathrm{a}_{\mathrm{p} \text {-in }}^{\mathrm{cr}}(\mathrm{f})$ used for the transition amplitude curve in Fig. 3.14, one can say that an inlet pressure pulsation for a fixed $\bar{p}_{\text {in }}$ is subcritical if

$$
\frac{a_{p-\text { in }}}{\bar{p}_{\text {in }}}<\frac{a_{p-\text { in }}^{\text {cr }}}{\bar{p}_{\text {in }}},
$$

and it is supercritical if

$$
\frac{a_{p-i n}}{\bar{p}_{\text {in }}}>\frac{a_{p-\text { in }}^{c r}}{\bar{p}_{\text {in }}} \text {. }
$$

An estimate of $\frac{a_{p-i n}^{c r}}{\bar{p}_{\text {in }}}$ at the frequencies investigated here may be obtained from the values reported in Table 3.1. In a fully condensing flow, where the exiting liquid is sufficiently 
sub-cooled and no vapor pockets exist immediately downstream of the condenser, the time-varying inlet mass flow rate, condensing-surface cooling conditions, and time varying pressure at a single point in the flow field (which, in this case, is a point on the inlet cross-section) are sufficient to fully specify the flow. This is because, if the condenser exit is sufficiently downstream of the all liquid zone, the pressure oscillations at the test-section exit are not associated with acoustic waves that reflect (see section 3.4.5), so they do not affect upstream liquid morphology in the flow condensation region. Therefore, the convention of Eqs. (3.14) - (3.15) is used in Figs. 3.14 - 3.15.

Alternatively, for better characterization of the effects of pulsations over the annular zone region of $0 \leq x \leq 40 \mathrm{~cm}$, one could use amplitude-frequency content characterizations of the fluctuations present in the representative pressure-difference signal $\Delta p_{40 \mathrm{~cm}}(t)$. For example, by using $X=\Delta p_{40 \mathrm{~cm}}(\mathrm{t})$ in Eqs. (3.11) - (3.12) for characterizing fluctuations, and defining the critical transition curve as $\mathrm{a}_{\Delta \mathrm{p}-40 \mathrm{~cm}}^{\mathrm{cr}}(\mathrm{f})$ in an alternate version of Fig. 3.14, one could re-state the conditions in Eqs. (3.14) - (3.15). In this alternative definition, one could say a pulsation is subcritical or supercritical if, respectively, the following is satisfied:

$$
\frac{a_{\Delta p-40 \mathrm{~cm}}}{\overline{\Delta p}_{40 \mathrm{~cm}}}<\frac{a_{\Delta \mathrm{p}-40 \mathrm{~cm}}^{\mathrm{cr}}}{\overline{\Delta p_{40 \mathrm{~cm}}}} \text { or } \quad \frac{a_{\Delta \mathrm{p}-40 \mathrm{~cm}}}{\overline{\Delta p_{40 \mathrm{~cm}}}}>\frac{a_{\Delta \mathrm{p}-40 \mathrm{~cm}}^{\mathrm{cr}}}{\overline{\Delta p}_{40 \mathrm{~cm}}}
$$

An estimate of $\frac{a_{\Delta p-40 \mathrm{~cm}}^{c \mathrm{c}}}{\Delta \mathrm{p}_{40 \mathrm{~cm}}}$ at the frequencies investigated is easily obtained from the values reported in Tables $3.1-3.2$.

Note that the amplitude of the inlet pressure fluctuations relative to the mean inlet pressure remains small for both subcritical and supercritical fluctuations and is not a true measure of its impact. It is the fluctuation amplitude's value relative to the small annular zone condenser pressure-difference (as in Eq. 3.16) that is indicative. This is why the alternative definition in Eq. (3.16) is preferable from the point of view of the flow physics in the annular zone. 
Considering the important role of the pressure-difference from the inlet to the heat-flux measurement location in the response of the flow, it is also possible to express the heat-flux enhancement shown in Fig. 3.15 in terms of $a_{\Delta p-40 c m}$ instead of in terms of $a_{p-i n}$ (see Fig. 3.17). Indeed, consideration of the flow dynamics according to classical flow physics suggests a qualitative correlation between the fluctuations in $\Delta \mathrm{p}_{40 \mathrm{~cm}}(\mathrm{t})$ and the dynamic bulk vapor volume flow rate (and by extension the dynamic vapor velocity fields) in the first $40 \mathrm{~cm}$ of the condenser. The vapor velocity flow fields which correlate with bulk vapor volume flow rate drive dynamic interfacial shear stress variations along the liquid-vapor interface in this region.

The relationship between $\mathrm{p}_{\text {in }}^{\prime}(\mathrm{t})$ and the dynamic volume flow rates which drive $\Delta \mathrm{p}_{40 \mathrm{~cm}}^{\prime}(\mathrm{t})$ is very complex and is governed by viscous, acoustic and other inertial effects which depend on the geometry of the test-section and the flow geometry immediately upstream of the test-section, along with the ability of the condensing film to absorb mechanical energy from the acoustic waves. Describing these effects in detail is beyond the scope of this dissertation, although some qualitative explanations are given later in section 3.4.5. The complexities are compounded by the fact that changes in flow direction and area at the test-section inlet immediately upstream of the $\mathrm{x}=0 \mathrm{~cm}$ location introduce their own acoustic waves and preclude the turbulent vapor flow entering the condenser from being considered "developed" until around $x=7.5 \mathrm{~cm}$.

The analogue of Fig. 3.15 for $\Delta p_{40 \mathrm{~cm}}(t)$ is shown in Fig. 3.17, where it may be seen that the frequency dependence of the heat flux enhancement has shifted from the relationship shown in Fig. 3.15. Notice particularly that the curve for $f_{P}=3.9 \mathrm{~Hz}$ is to the left of the other curves in Fig. 3.17, whereas it is to the right of the other curves in Fig. 3.15. This is because at this lower frequency, the amplitude of the inlet pressure fluctuations at $f_{P}$ is significantly more than the amplitude of the pressure-difference between the $x=0 \mathrm{~cm}$ and the $x=40 \mathrm{~cm}$ locations in the condenser. For the higher values of $f_{p}$, the values of $\mathrm{a}_{\mathrm{p}-\mathrm{in}}$ and $\mathrm{a}_{\Delta \mathrm{p}-40 \mathrm{~cm}}$ are more similar, indicating that the hydrodynamics of the flow are significantly frequency-dependent, although the shape of the curves in Fig. 3.15 and 
Fig. 3.17 and the levels of enhancement in $\overline{q_{40 \mathrm{~cm}}^{\prime \prime}}$ achieved are similar for all frequencies $f_{P}$ investigated.

A similar frequency effect was observed for all-annular condensing flows in Figs. $2.21-2.22$ of chapter 2. It is expected that, as reported in Fig. 2.23 of chapter 2 for all-annular condensing flows, for these fully condensing flows $\mathrm{a}_{\mathrm{p}-40 \mathrm{~cm}}$ also directly correlates with $\overline{\Delta \mathrm{q}_{40 \mathrm{~cm}}^{\prime \prime}} \equiv \overline{\mathrm{q}_{40 \mathrm{~cm}}^{\prime \prime}}-\left.\overline{\mathrm{q}_{40 \mathrm{~cm}}^{\prime \prime}}\right|_{\mathrm{N}-\mathrm{F}}$.

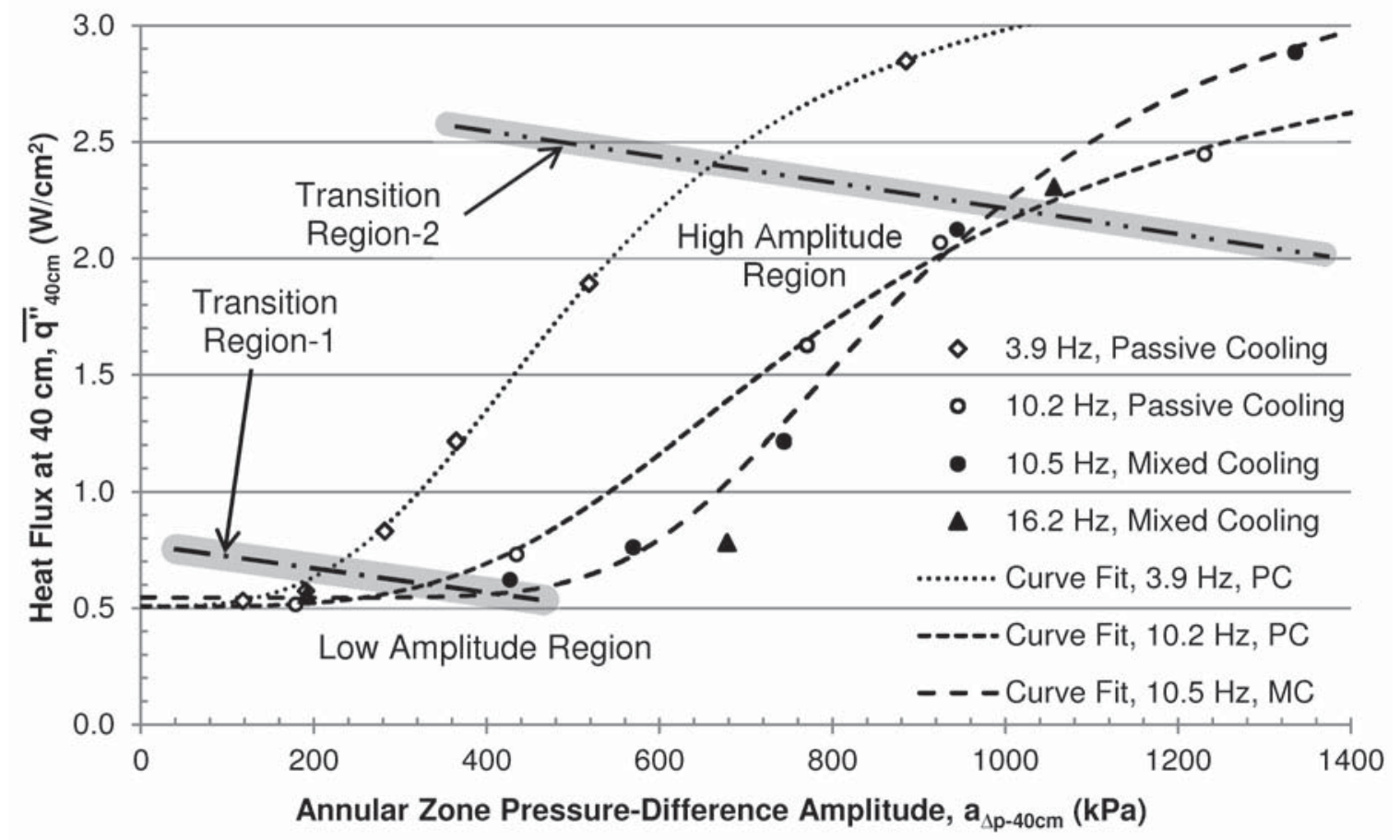

Figure 3.17. Heat-flux enhancement vs. $\Delta p_{40 \mathrm{~cm}}$ amplitude chart for different pulsator frequencies. The behavior is similar for two different condensing surface cooling conditions (Passive Cooling, PC, and Mixed Cooling, MC).

The mechanical energy carried by the inlet pressure fluctuations increases with their dominant frequency $f_{p}$, the amplitude of absolute pressure fluctuations at this value of $f_{p}$, and the amplitude of bulk volume flow rate fluctuations at this value of $f_{p}$. While the 3.9 Hz IF-HA cases in Figs. 3.15 and 3.17 have a lower value of $f_{p}$ and $a_{\Delta p-40 \mathrm{~cm}}$, they have a significantly higher value of $a_{p-i n}$, so the energy-efficiency of the heat-flux enhancement may depend on competing phenomena. A concurrent measurement of inlet 
pressure $p_{i n}^{\prime}(t)$ and volume flow rate $Q_{i n}^{\prime}(t)$ fluctuations at $f_{p}$ at or slightly upstream of the $\mathrm{x}=0 \mathrm{~cm}$ location of Fig. 3.1 is necessary to evaluate the physical energy entering the test-section as a result of the imposed fluctuations ( or $Q_{\text {in }}^{\prime}(t)$ may be calculated from mass flow rate fluctuations $\dot{M}_{\text {in }}^{\prime}(\mathrm{t})$ ). The mass flow rates in Fig. 3.3 are not sufficiently accurate, and their measurement is too far away from the test-section, to allow computation of the mechanical energy entering the test-section from the imposed pressure fluctuations, so no such computations have been included here.

Figures 3.14 - 3.15 and 3.17 represent the entire data-matrix of relevant data from the experiments being reported. Other cases which do not appear in Tables 3.1 - 3.2 had instrumentation or flow control problems which made them less useful to the purposes of this chapter. Some of the highest amplitude imposed fluctuation cases were omitted because of limitations in the measurement range of DPT-1 in Fig. 3.1. Some low amplitude imposed fluctuation cases were omitted because they would have been redundant. Other cases that were affected by system start-up or transients have also been omitted.

Of the data matrix in Tables 3.1 - 3.2, only a few representative flow realizations from Tables 3.1 - 3.2, namely cases P07, P05, and P02, which had the passive cooling condition, have been graphically discussed in detail. These cases were chosen because they represent the three types of imposed fluctuations (IF-LA, IF-TA, and IF-HA), are near the middle of the frequency range investigated, and represent the simpler of the cooling conditions.

\subsubsection{Non-annular regimes of fully condensing flows}

For most fully condensing flows discussed above, the overall heat removal rate between

the inlet and the exit of the test-section is, approximately, the latent heat released $\left(\overline{\mathrm{M}}_{\mathrm{in}} \cdot \mathrm{h}_{\mathrm{fg}}\right)$ plus significant liquid sub-cooling needed to fully condense the flow because of the high thermal resistance associated with the non-annular portion of the flow where the effective liquid layer is thick. It is hypothesized that inlet pressure work associated with 
fluctuations is significantly smaller and affects mainly the inlet vapor superheat, which is a small fraction of the total heat removed from the fluid during condensation (see caption of Table 3.2).

By significant sub-cooling of the liquid below saturation temperature in the non-annular zone, it is meant that the amount of liquid that is below saturation temperature and the amount by which the adjacent condensing-surface temperature is below saturation temperature are significantly higher relative to the annular zone. Therefore, any increase in the heat flow rate over the annular portion (up to $x_{A}$ ) of the flow associated with a decrease in thermal resistance may require a significant increase in the liquid sub-cooling heat removal rate (see parameter $\mathrm{Ja}_{\mathrm{L} \text {-exit }}$ in Table 3.2) associated with increased thermal resistance for the remaining non-annular portion (particularly if this zone is significant in length). In Fig. 3.4, for $f_{P}=3.9 \mathrm{~Hz}$ excitation (case P14), the non-annular zone's thermal resistance was significantly increased relative to case NF3, and the annular length was significantly reduced, resulting in a significantly higher $\mathrm{Ja}_{\mathrm{L} \text {-exit }}$. However, for case P02, the length of the annular regime relative to case NF3 did not change as much, resulting in a significantly smaller increase in $\mathrm{Ja}_{\mathrm{L}-\text { exit }}$.

Because of the varying contributions arising from the non-annular zone's sub-cooling needs and its complex oscillatory morphology, one cannot easily develop a generally valid comparison between imposed fluctuation cases and the corresponding no-imposed fluctuation (N-IF) natural case with regard to the total heat removed over the entire test-section (consisting of annular and non-annular zones).

Additionally, the vapor quality at the end of the annular regime may vary such that even if the heat-flux is higher in the annular regime (such as in the IF-HA cases), the value of $x_{A}$ may not decrease by much. The value of $x_{A}$ may even increase with increasing fluctuation amplitude for IF-LA and IF-TA cases as shown in Fig. 3.18. It is possible that low amplitude fluctuations help in suppressing high amplitude interfacial waves in the downstream regions of the condenser which would otherwise break or reach the top of 
the flow channel, thus allowing the annular flow regime to persist into regions of lower vapor quality than are possible with the N-IF flows. Since the heat-flux enhancement is also lower for these cases (see Figs. 3.15 and 3.17), the vapor quality is not reducing much more rapidly with distance from the condenser inlet than for the corresponding N-IF flows. However, when the amplitude of imposed pressure fluctuations at $f_{P}$ becomes high enough, the reduction in vapor quality at downstream locations due to increased heat-flux in the annular regime wins over any stabilization effect that the pressure fluctuations would otherwise have had on the flow - leading to a general decrease in the values of $x_{A}$ with increasing values of $a_{\Delta p-40 c m}$ for the IF-HA cases in Fig. 3.18.

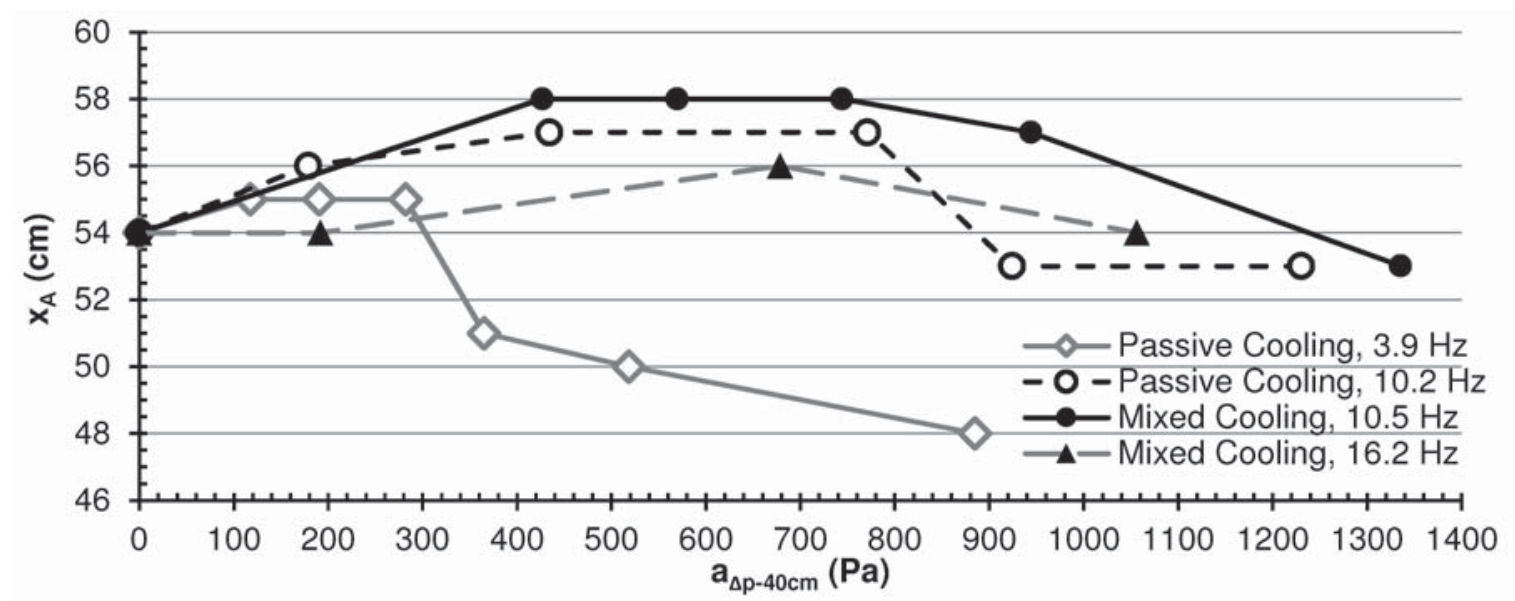

Figure 3.18. Approximate length of the annular flow regime for the cases in Table 3.1, organized by the imposed pulsation frequency $f_{P}$ and the cooling conditions, as the amplitude of imposed pressure-difference fluctuations increases.

It is important to note that, although analysis of the general trends of $x_{A}$ in Fig. 3.18 is instructive, the uncertainties in these $x_{A}$ values are high because they were estimated by eye. Therefore, it is not useful to use details of individual curves in Fig. 3.18 to draw conclusions regarding the nature of the flow. The passive cooling curves appear to have an inflection point after the values of $\mathrm{x}_{\mathrm{A}}$ start decreasing. This might be due to the increasing surface temperatures of the IF-HA cases as shown in Fig. 3.7, or it could be a result of another phenomenon or of inconsistency in the estimation of the $\mathrm{x}_{\mathrm{A}}$ values. 
The heat loads in the vicinity of $x_{A}$ do increase with increasing values of $a_{\Delta p-40 c m}$, but at a much lower rate than they do in the annular flow regime. This is shown in Fig. 3.19.

The heat-flux values $\overline{q_{55 \mathrm{~cm}}^{\prime \prime}}$ are from heat-flux meter HFX-2 in between the bottom of the condensing plate of Fig. 3.1a and the thermoelectric cooler TEC-6 of Fig. 3.1b. The sensor cross-sectional area through which heat flow is measured is $3.81 \mathrm{~cm}$ by $3.81 \mathrm{~cm}$; this sensor was sized to match the cross-sectional area of TEC-6, which is about $4 \mathrm{~cm}$ by $4 \mathrm{~cm}$. It is a solid-state thermopile type sensor, like HFX-1 in Fig. 3.1b.

Since the 10 TECs are evenly distributed over the $100 \mathrm{~cm}$ length of the active region of the condensing plate, each one removes heat from approximately $10 \mathrm{~cm}$ of the length of the condensing surface in the direction of flow. Thus $\overline{q_{55 \mathrm{~cm}}^{\prime \prime}}$ represents heat removal from approximately $x=50 \mathrm{~cm}$ to $x=60 \mathrm{~cm}$ at the top surface of the condensing plate. The values of $\overline{q_{55 \mathrm{~cm}}^{\prime \prime}}$ are only useful for qualitative purposes because axial and lateral heat conduction are significant in the $12.7 \mathrm{~mm}$ thick by $50.8 \mathrm{~mm}$ wide condensing plate (which extends to the sides underneath the plastic cover beyond the flow channel width).

The reason that the mixed cooling cases in Fig. 3.19 show such a marked increase in $\overline{q_{55 \mathrm{~cm}}^{\prime \prime}}$ for the high amplitude cases is because of the use of TEC- 6 to hold surface temperature constant at $x=58.5 \mathrm{~cm}$. Because of the thermal resistance of the condensing plate, the flows that exhibit higher heat-flux from the fluid into the top surface of the condensing plate require more heat to be removed by the TEC to maintain the desired condensing surface temperature.

Conversely, one might conclude that for the passive cooling condition cases, the increase in surface temperatures in the region around $x=55 \mathrm{~cm}$ with increased heat-flux into the top of the condensing plate (caused by the thermal resistance of the condensing plate coupled with the passive cooling condition) acts to oppose the flow of additional heat into the condensing plate by reducing the fluid-to-surface temperature difference, thus moderating the amount of heat-flux enhancement that can be realized at this location. 


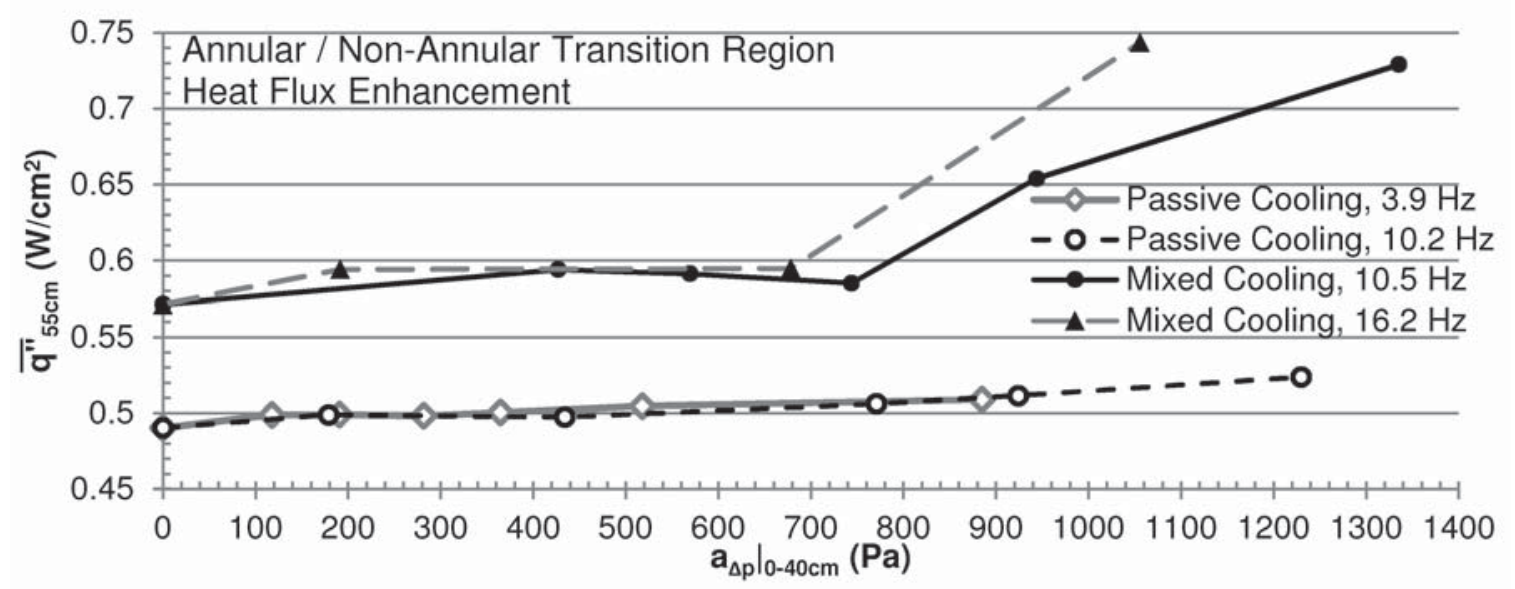

Figure 3.19. Heat-flux removed from the region of the test-section where the flow is transitioning to non-annular regimes. This is not heat-flux removed from the fluid at the condensing surface. It provides a rough qualitative estimate of the heat removed from the condensing surface over $50 \mathrm{~cm} \leq x \leq 60 \mathrm{~cm}$. Note the contrast between the "passive" and "mixed" cooling conditions.

\subsubsection{All-annular regimes in innovative condensers employing partially condensing flows}

The above-reported benefits of the annular zone's supercritical sensitivity to inlet pressure fluctuations can be better exploited in new system designs of the type presented in chapter 2 . These systems incorporate condensers which only involve partially condensing annular flows within the condenser. For such condensers, preliminary testing suggests that suitable designs of their exit geometry and appropriate operating procedures will make their annular zone's responses to imposed fluctuations retain the mechanisms (see section 3.4.5) which lead to the heat-flux enhancements discussed above for fully condensing flows. This would allow these innovative condensers to operate at much higher heat loads for the same condenser length, width, and height.

Chapter 2 demonstrates high enhancement in local heat-flux attained with an all-annular flow condenser at $\mathrm{f}_{\mathrm{P}} \approx 28.5 \mathrm{~Hz}$ and moderate increase in the overall rate of condensation due to the imposed pulsations at $\mathrm{f}_{\mathrm{P}}=10.6 \mathrm{~Hz}$ and $\mathrm{f}_{\mathrm{P}} \approx 28.5 \mathrm{~Hz}$, but more work is needed to fully explore the potential of this type of flow condenser. 


\subsubsection{The role of amplitude-frequency content of fluctuations in attaining different quasi-steady flows}

To better understand the role of amplitude and frequency content of fluctuations on attaining different steady-in-the mean flows, we look at 5 second long dynamic signals (acquired at DAQ rate of $2000 \mathrm{~Hz}$ ) for: inlet pressure $\mathrm{p}_{\mathrm{in}}(\mathrm{t})$, heat-flux $\mathrm{q}_{40 \mathrm{~cm}}^{\prime \prime}(\mathrm{t})$, and the test-section's annular regime pressure-difference $\Delta \mathrm{p}_{40 \mathrm{~cm}}(\mathrm{t})$. These dynamic signals are obtained at certain discrete time locations in the longer duration lower DAQ rate time-histories (e.g. as shown in Figs. 3.5 - 3.6) of these variables. The frequency domain plots (obtained by Fast Fourier Transforms, FFT implementation) of $\left[p_{i n}^{\prime}(t) \equiv p_{i n}(t)-\bar{p}_{i n}\right]$, $\left[\Delta \mathrm{p}_{40 \mathrm{~cm}}(\mathrm{t})\right]$, and $\left[\mathrm{q}_{40 \mathrm{~cm}}^{\prime \prime}(\mathrm{t})\right]$ are respectively shown in Figs. $3.20-3.22$.

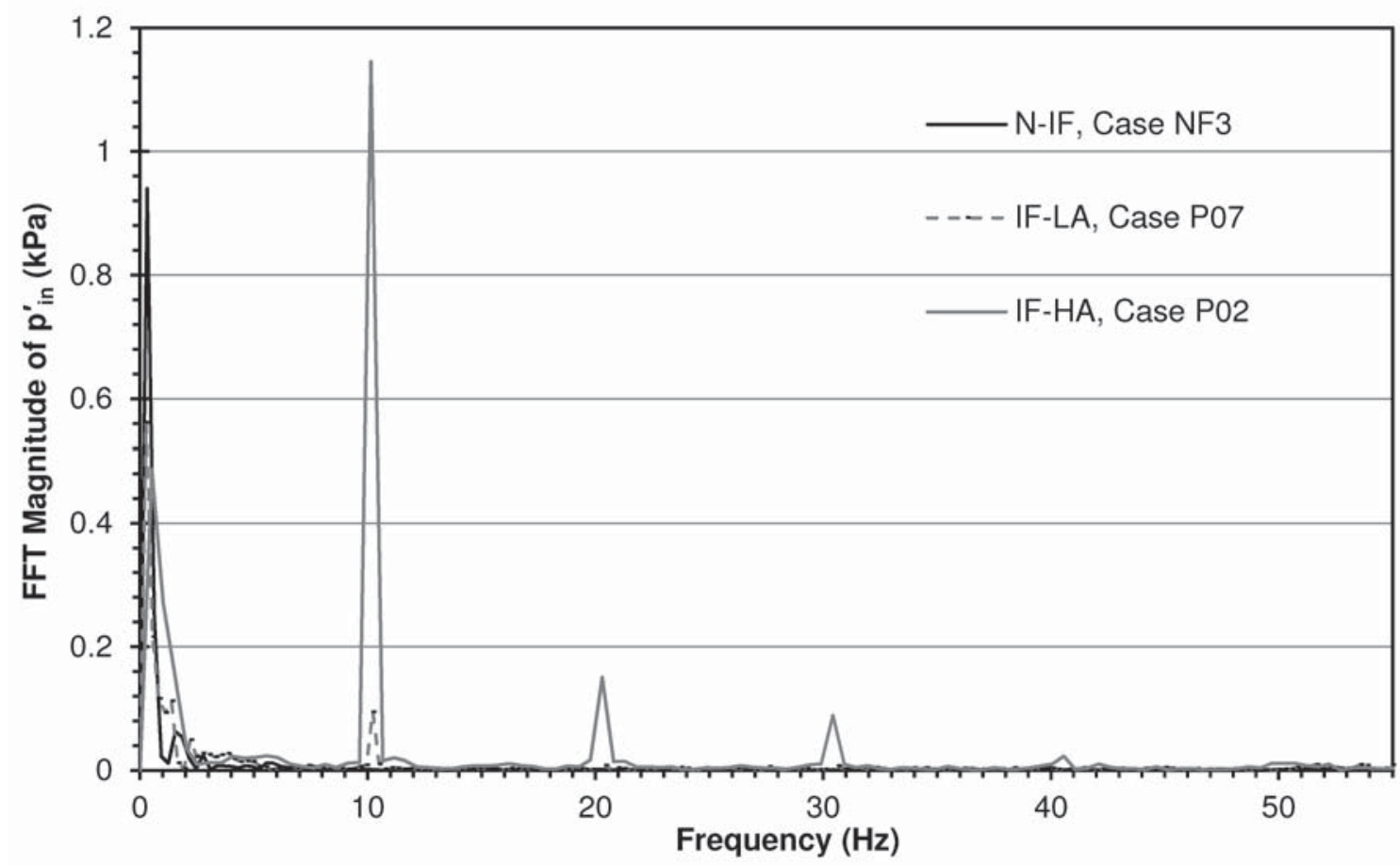

Figure 3.20. FFT magnitude plots of inlet pressure fluctuations $p_{i n}^{\prime}(t)$. These are representative of the cases from Table 3.1 indicated in the legend, and they were taken under the "passive" cooling condition.

It is clear from Fig. 3.20 that the pulsator induced frequencies $(10.2 \mathrm{~Hz}$ and its harmonics) have a significant presence for the imposed fluctuation cases but not for the no-imposed fluctuation case. As observed from the time-varying heat-flux curves (shown 
in Fig. 3.13) and film thickness curve from Eq. (3.13) (shown in Fig. 3.16), both the heat-flux and instantaneous condensate film thickness for the IF-HA cases oscillate around their new means with the principal driving frequency of $10.2 \mathrm{~Hz}$, but they do so in the presence of amplitudes associated with the first $(20.4 \mathrm{~Hz})$ and second $(30.6 \mathrm{~Hz})$ harmonics. Note that, because the harmonics in the heat-flux FFT (Fig. 3.22) are significant compared with the fundamental fluctuation amplitude, $a_{q^{\prime \prime}-40 \mathrm{~cm}}$, they enable the non-sinusoidal nature (with respect to the nearly steady N-IF values) of the IF-HA cases' heat-flux vs. time relationship in Fig. 3.13. The aforementioned dynamics are such that, as the amplitudes of the imposed pulsations increase, they always lead to a significant percentage increase in the mean heat-flux values of ${\overline{q_{40 \mathrm{~cm}}}}$ (see Table 3.2).

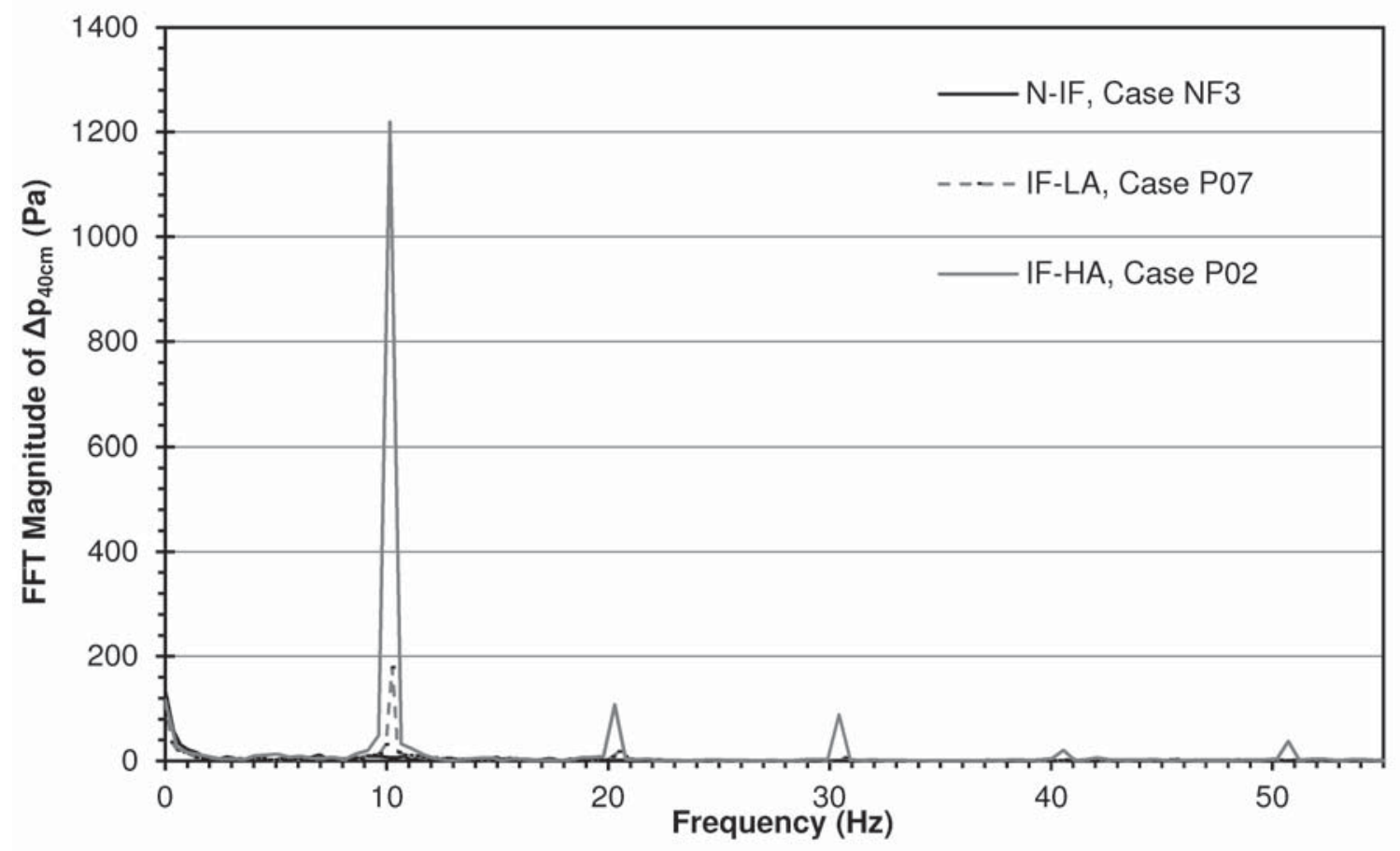

Figure 3.21. FFT magnitude plots of differential pressure across the first $40 \mathrm{~cm}$ of the test-section. These are representative of the cases from Table 3.1 indicated in the legend, and they were taken under the "passive" cooling condition.

The underlying hydrodynamics is better illustrated by the presence of first $(20.4 \mathrm{~Hz})$ and second $(30.6 \mathrm{~Hz})$ harmonics in the FFT of $\left[p_{i n}^{\prime}(t) \equiv p_{i n}(t)-\bar{p}_{i n}\right]$ in Fig. 3.20 and FFT of $\left[\Delta p_{40 \mathrm{~cm}}(\mathrm{t})\right]$ in Fig. 3.21. Since the first and second harmonics in the inlet pressure 
fluctuation magnitudes are small compared to their mean $($ at $f=0)$ and their values at the principal (fundamental) frequency $\left(f=f_{p}\right)$, a similar principal frequency dominance is expected for the pressure-difference that drives the single-phase vapor flow between some point upstream of the test-section (but downstream of the pulsator in Fig. 3.2) and the test-section inlet. Therefore, as confirmed by Fig. 3.3, the resulting inlet mass flow rate $\dot{M}_{\text {in }}(t)$ also has an insignificant presence of harmonics. What is significant is that, for high amplitude cases, the first and second harmonics in Fig. 3.21 (the FFT of $\Delta \mathrm{p}_{40 \mathrm{~cm}}(\mathrm{t})$ ) are comparable in magnitude to the mean pressure-difference $\overline{\Delta p}_{40 \mathrm{~cm}}$ (magnitude of $\overline{\Delta \mathrm{p}}_{40 \mathrm{~cm}}$ indicated by the FFT amplitude at $\mathrm{f}=0$ ).

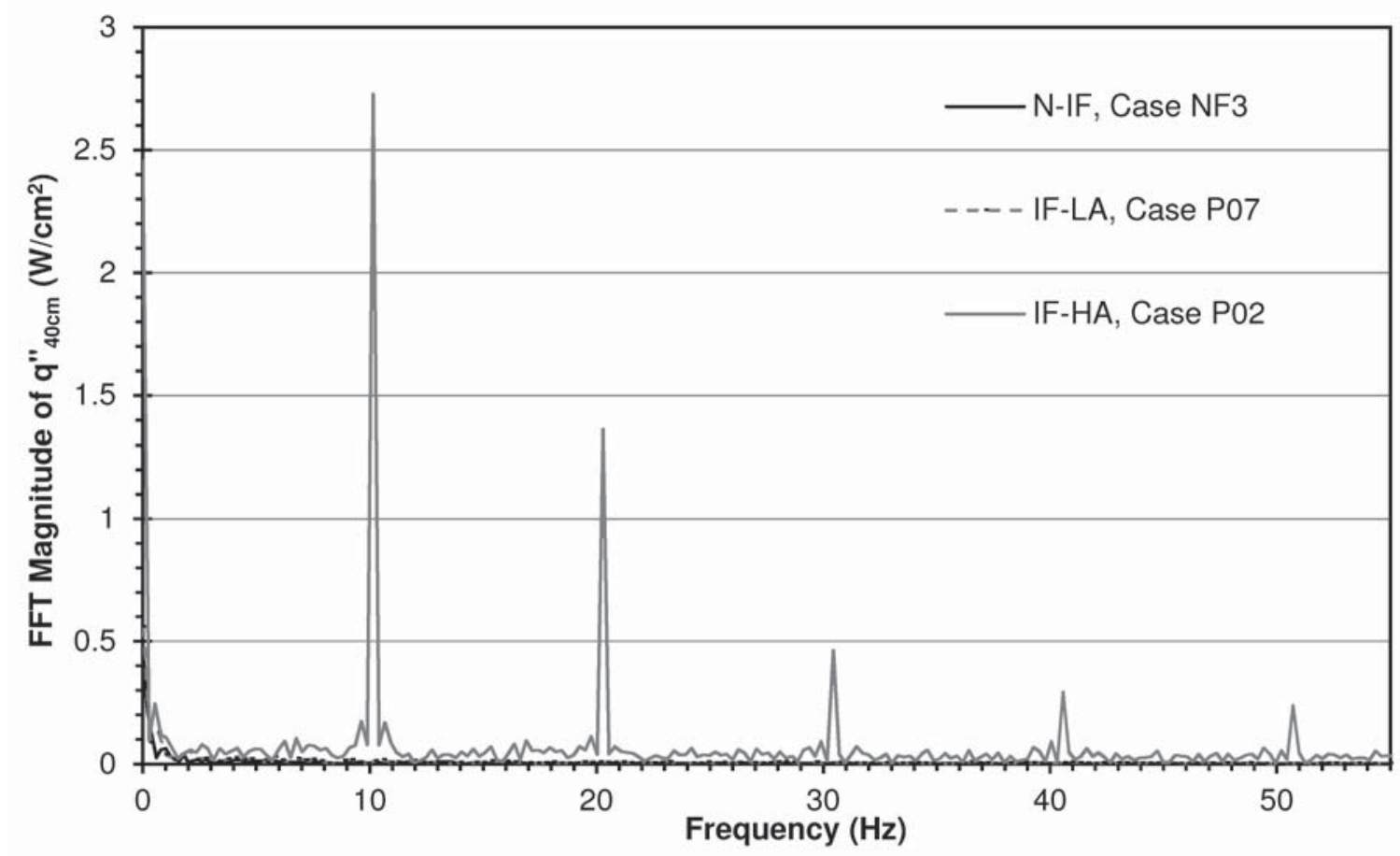

Figure 3.22. FFT magnitude plots of heat-flux at the $x=40 \mathrm{~cm}$ location in the test-section. These are representative of the cases from Table 3.1 indicated in the legend, and they were taken under the "passive" cooling condition.

Next, dynamic time-varying annular zone outlet pressure data $p_{40 \mathrm{~cm}}(t) \equiv p_{L}(x=40 \mathrm{~cm}, t) \cong p_{v}(x=40 \mathrm{~cm}, t)$ is obtained (and shown in Fig. 3.16) from the relationship $\mathrm{p}_{40 \mathrm{~cm}}(\mathrm{t}) \cong \mathrm{p}_{\mathrm{in}}(\mathrm{t})-\Delta \mathrm{p}_{40 \mathrm{~cm}}(\mathrm{t})$ and the measured values of time-varying inlet pressure and pressure-difference. From Fig. 3.23, below, it is clear that amplitudes 
of $\mathrm{p}_{40 \mathrm{~cm}}^{\prime}(\mathrm{t})$ at the first and second harmonics of $\mathrm{f}_{\mathrm{p}}$ are significant compared with the amplitude of $\mathrm{p}_{40 \mathrm{~cm}}^{\prime}(t)$ at $f_{\mathrm{p}}$. This means that, along with nearly sinusoidal mass flow rate fluctuations at the inlet, the presence of first and second harmonics (over and above the fundamental frequency, $f_{p}$ ) in the annular zone's pressure fluctuations at $x=40 \mathrm{~cm}$ plays a critical role in the determination of the changed structure of the wavy annular flow (as seen in representative Videos 3-1 - 3-3 provided on the accompanying data disc) between $0 \leq x \leq 40 \mathrm{~cm}$. This wave-structure induced heat-flux enhancement mechanism and the source of the aforementioned harmonics are discussed below in section 3.4.5.

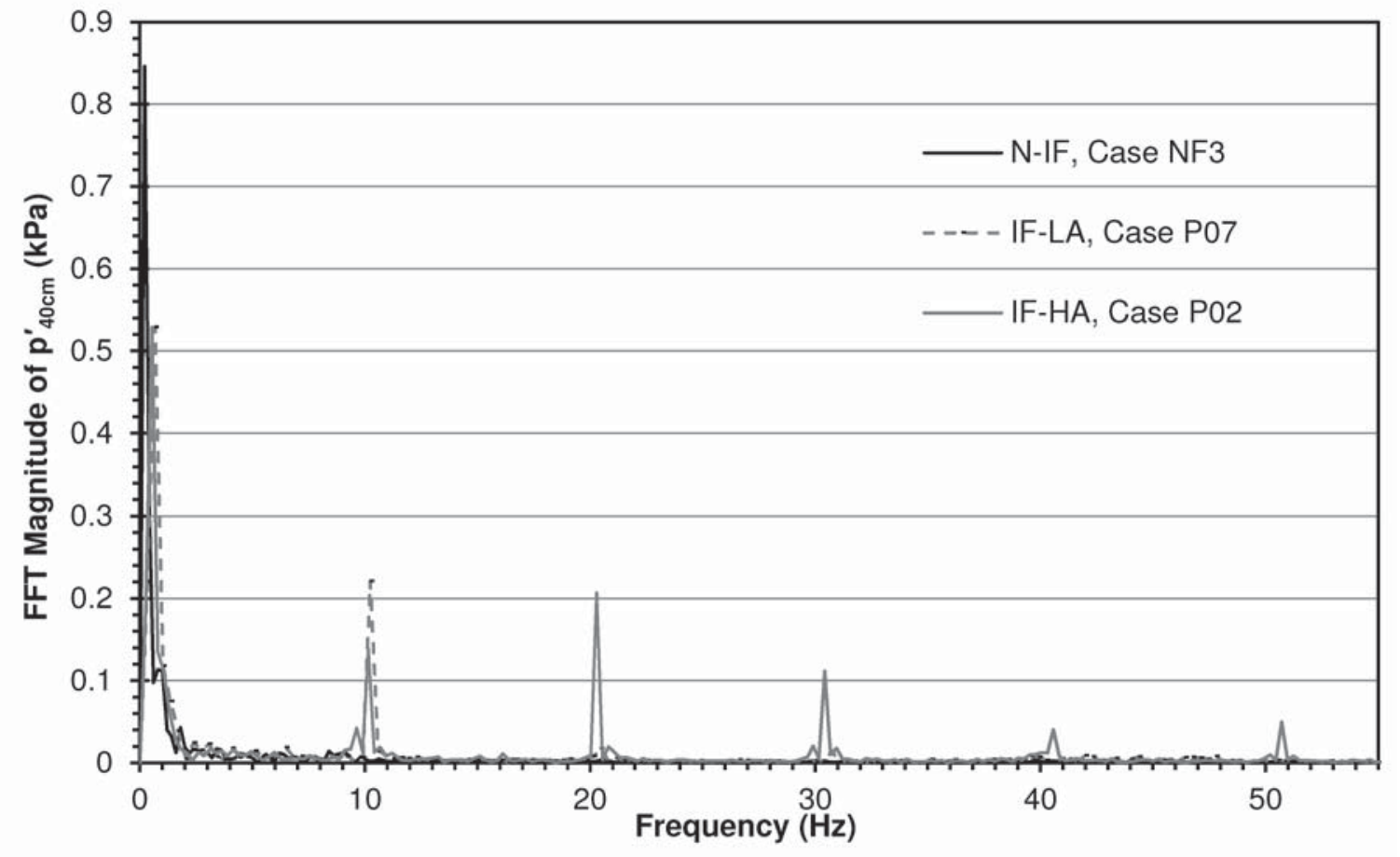

Figure 3.23. FFT magnitude plots of estimated fluctuations in absolute pressure at the $x=40 \mathrm{~cm}$ location in the test-section. These are representative of the cases from Table 3.1 indicated in the legend, and they were taken under the "passive" cooling condition. 


\subsubsection{Annular zone heat-flux enhancement mechanisms}

Noise-sources at locations upstream of the condenser inlet (such as the pulsator in Fig. 3.2) create pressure and mass flow rate pulsations at the condenser inlet. However, the quasi-steady nature of the flow rate and pressure pulsations that are eventually attained at the inlet and over a well-defined annular region $0 \leq \mathrm{x} \leq \mathrm{x}^{*}$ of the condenser (where $\mathrm{x}^{*}<\mathrm{x}_{\mathrm{A}}(\mathrm{t})$ for all times and for a range of experimental conditions) depends on whether or not there is a significant presence of standing acoustic waves in the time-varying vapor flow and how these acoustic waves interact with the slower moving interfacial waves on the liquid condensate.

For the data matrix in Fig. 3.14, $x^{*}=40 \mathrm{~cm}$ defines the outlet of an annular zone $0 \leq \mathrm{x} \leq \mathrm{x}^{*}$. Standing acoustic waves are a manifestation of transient compressibility effects (manifested over short durations, $\Delta \mathrm{t}_{\mathrm{C}} \approx 1 / \mathrm{f}_{\mathrm{P}}$ ) in the low Mach number vapor flow. A relevant measure of this for the time-varying vapor control volume " $\mathrm{CV}(\mathrm{t})$ " within the annular region $0 \leq x \leq x^{*}$ is the ratio of the rate of change of stored mass in the

control-volume $\left(\int_{C V(t)} \frac{\partial \rho}{\partial t} \cdot d v o l\right)$ to the mean inlet mass flow rate $\overline{\mathbf{M}}_{\text {in }}$. A non-dimensional measure of this ratio was defined as $\mathrm{TCND}_{\text {Total }}$ in section 3.2 , following the approach in [41].

There is sufficient experimental evidence to indicate that compressibility effects are negligible for IF-LA cases, and they start becoming significant as inlet pressure pulsations are increased beyond Transition Region-1 in Fig. 3.15 to reach IF-HA cases. There are several experimental observations that support the hypothesis that it is the standing acoustic waves in the vapor phase, resulting from superposition of forwardand-backward moving acoustic waves over the annular region (over $0 \leq \mathrm{x} \leq \mathrm{x}_{\mathrm{A}}(\mathrm{t})$ ), that contribute to the compressibility effect. These observations also indicate that this effect is small for the low Mach number flow if the acoustic waves just pass through in the forward direction. First it should be noted that IF-LA cases in Figs. 3.14 - 3.15 are close to the behavior of the N-IF (or steady) case for which compressibility effects are negligible, and whose annular zone liquid-vapor interface in Fig. 3.1a is stable. 
As a result, when acoustic wave amplitudes start becoming significant in the vicinity of Transition Region-1 (Fig. 3.14), the forward moving acoustic waves in the annular zone over $x \leq x_{A}(t)$ start reflecting from the liquid-vapor interface which separates the vapor from the higher acoustic impedance of the non-annular region, which is made up of a higher acoustic impedance (significantly smaller changes in the liquid density) and predominantly liquid zone, over $x_{A}(t) \leq x \leq L$ in Fig. 3.1a.

As discussed in [42], standing waves (say in longitudinal pressure pulsations) in the vapor phase resulting from the interference of the forward-and-backward moving acoustic waves can manifest several harmonics (frequencies $2 \cdot f_{p}, 3 \cdot f_{p}$, etc.) that are multiples of the principal (fundamental) frequency $f_{p}$. The resulting oscillatory energy spatially distributes itself in patterns that will contain approximate nodes and anti-nodes within the annular vapor zone. Although acoustic wave propagation in the present context is somewhat dependent on the nature of the underlying mean steady flow in the annular vapor region, the aforementioned knowledge [42] of acoustic waves in a medium where its propagation is independent of the base flow is not sufficiently applicable to the present case for elucidating its underlying physics. This is because the interfacial waves can interact with acoustic waves to significantly alter or choose the location of interior nodes and anti-nodes. However, one can still assume that acoustic waves are approximately governed by a wave equation [43] that allows wave propagation along any directed unit vector, in both forward and backward directions.

For a representative IF-HA fully condensing flow case, Fig. 3.22 shows the FFT magnitude of the time-varying heat-flux $q_{40 \mathrm{~cm}}^{\prime \prime}(\mathrm{t})$ from Fig. 3.13. It clearly establishes that the amplitudes at the harmonics (frequencies $2 \cdot f_{p}, 3 \cdot f_{p}$, etc.) are significant relative to the amplitude at the principal frequency $f_{p}$. Similarly the FFT magnitude of the time-varying annular zone pressure fluctuations $\left(\mathrm{p}_{40 \mathrm{~cm}}^{\prime}(\mathrm{t})\right.$ in Fig. 3.16) is shown in Fig. 3.23, and it shows a significant presence of harmonics relative to its amplitude at frequency $f_{P}$, which is $10.1-10.2 \mathrm{~Hz}$. The presence of these harmonics is consistent with the hypothesis that 
standing waves are present and is suggestive of the critical role standing waves may play in interacting with the interfacial waves that activate the mechanisms that lead to heat-flux enhancement.

Furthermore the FFT magnitude (Fig. 3.20) of the experimentally measured time-varying inlet pressure at $x=0$ and, at least for the IF-HA cases near Transition Region- 1 of Fig. 3.14, the higher acoustic impedance liquid in the downstream side of the interface near $\mathrm{X}=\mathrm{x}_{\mathrm{A}}(\mathrm{t})$ suggest that longitudinal standing waves will form an approximate anti-node in pressure at the inlet as well as near $x=x_{A}(t)$. Note that acoustics dictates that anti-nodes in pressure occur where there are nodes in velocity. However, depending on the IF-HA case being considered, the absolute pressure $p_{40 \mathrm{~cm}}(t)$ variations at the measurement point $x^{*}=40 \mathrm{~cm}$ may indicate that the point is either closer to a node or an anti-node in pressure. For the absolute pressure variation $\mathrm{p}_{40 \mathrm{~cm}}(\mathrm{t})$ shown in Fig. 3.16, the FFT magnitudes in Fig. 3.23 suggest that $x^{*}=40 \mathrm{~cm}$ is closer to a node. How these nodes and anti-nodes are formed while interacting with interfacial waves is not known at this time. Therefore it is hard to use simple acoustics analogies (such as an open-closed organ in [42]) to suggest the reasons for why mainly the first and second harmonics get selected in the interfacial wave structure.

It has also been experimentally verified by us (but not reported here for brevity) that the downstream resistance to the passage of acoustic waves is significantly reduced for certain partially condensing flows with arrangements that allow relatively unrestricted flows of the vapor and the liquid from $x=x^{*}$ to the test-section exit, and then to a partially filled liquid plenum downstream of the condenser where the vapor and the liquid flows are separated, and vapor pressure pulsations are significantly damped. For these special partially condensing flow experiments, acoustic waves primarily pass only in the forward direction over $0 \leq x \leq x^{*}$; they do not return from the exit at $x=L$. Inlet mass flow rate, cooling conditions, and inlet pressure as obtained for one of the cases reported here were first used to attain a quasi-steady N-IF case over $0 \leq x \leq x^{*}$ for this partially condensing flow, and then pulsations were imposed at the same pulsator frequency and 
similar valve opening $\mathrm{V}_{\mathrm{P}}$ (see Fig. 3.2) associated with one of the corresponding IF-HA cases.

It was found that the dynamic response of the heat-flux $\mathrm{q}_{40 \mathrm{~cm}}^{\prime \prime}(\mathrm{t})$ for the above-described partially condensing IF-HA case remained similar to the N-IF case (i.e., heat-flux enhancements, as shown in Fig. 3.6, did not occur for this IF-HA case relative to its N-IF case. This supports the hypothesis that the compressibility effects are important only for cases involving significant vapor phase pressure pulsations which form standing acoustic waves.

More importantly, for the flow in the annular regime $0 \leq x \leq x^{*}$, several important conclusions can now be made to assist in the development of proper computational simulation capabilities for this zone.

For the IF-HA cases, the nature of the passage of acoustic waves through the downstream regions at $\mathrm{x} \geq \mathrm{x}^{*}$ has an impact on the flow in the upstream annular region $\left(0 \leq \mathrm{x} \leq \mathrm{X}^{*}\right)$ of interest. It should also be noted that interfacial waves in the annular condensing flow regime can, at any time and at any one location, move only in one direction, as they are governed by a first order quasi-linear wave equation with single characteristics (see governing equation and wave results in [41]).

Since the cases considered here involve mean forward mass flow of the liquid condensate, interfacial waves typically retain a forward positive velocity and they do not reflect at the $\mathrm{x}=\mathrm{x}^{*}$ boundary of the annular zone $\left(0 \leq \mathrm{x} \leq \mathrm{x}^{*}\right)$ of interest. Hence, as far as the experimentally indicated presence of harmonics in the pressure field over the annular zone $0 \leq \mathrm{x} \leq \mathrm{x}^{*}$ is concerned, transient compressibility wave effects arising from standing waves (forward and backward moving acoustic waves) are causative in bringing the downstream information upstream.

Despite this, as expected and discussed below, the immediate cause of condensate 
thinning and heat-flux enhancements is related to a more complex interaction between the interfacial waves, the aforementioned acoustic waves, and the "sticking" phenomenon described in section 2.3.5. Because of this immediate cause, for a range of amplitudes between Transition Region-1 and Transition Region-2 marked in Fig. 3.15, it is expected that unsteady low Mach number vapor and liquid flows in a region within the annular zone $\left(0 \leq x \leq x^{*}\right)$ can also be approximately modeled as incompressible (with respective constant densities of $\rho_{V}$ and $\rho_{L}$ ) provided the effects arising from acoustic waves in the vapor phase are accounted for by appropriate additional information coming from prescribed boundary conditions.

For example, experimentally measured values of time-varying (with harmonics) inlet $(x=0)$ and outlet $\left(x=x^{*}\right)$ cross-sectional pressure prescriptions along with time-variations (if any) in condensing-surface temperatures may be one set of boundary condition information that will work with the incompressible vapor assumption.

Time-varying values of inlet mass flow rate may be substituted for the time-varying inlet pressure prescription (with due care given to the measurement of harmonics) at $x=0$ in this set of boundary conditions.

In the language of computational physics (to be reported in a forthcoming paper that corrects some implementation errors in [36] and [38]), for the IF-HA cases, the vapor-phase acoustic and interfacial waves impart "elliptic sensitivity" — or sensitivity to downstream conditions - to the unsteady and incompressible problem (or governing equations) for the annular regime. However, "elliptic sensitivity" for the IF-LA cases can be ignored. Therefore, low amplitude pulsations behave closer to the approximately wave-free and steady flow problem which only exhibits a "parabolic" behavior (i.e. the annular zone behavior is not dependent on downstream conditions) for which one only needs parabolic boundary conditions (time-varying values of inlet mass flow rate, condensing-surface temperature, and pressure at any "one point" in the flow field) for simulation purposes. 
As the imposed inlet pressure fluctuation amplitude increases from Transition Region-1 to Transition Region-2 in Fig. 3.15, the flow visualization videos (see accompanying data disc for sample IF-HA Videos 3-2 - 3-3) indicate that the liquid-vapor interface starts showing vigorous oscillations and preferential shifts in its mean location. At this point, the downstream non-annular regime (predominantly liquid with vapor pockets) of $\mathrm{X}>\mathrm{X}_{\mathrm{A}}(\mathrm{t})$ is no longer stagnant but exhibits significant flow oscillations - presumably due to spring-like compressibility of the vapor pockets leading to oscillatory liquid motion and interface oscillations at $\mathrm{x}=\mathrm{x}_{\mathrm{A}}(\mathrm{t})$, shown in Videos 3-2 - 3-3 (see accompanying data disc).

The time-varying liquid mass that directly feeds into the non-annular zone through its connectivity to the annular zone liquid condensate motion (see Fig. 3.1a) also contributes to the motion in the non-annular regime. These oscillations are expected to change the percentage of reflected and transmitted energy associated with the acoustic waves that are incident on the liquid-vapor interface at $\mathrm{X}=\mathrm{x}_{\mathrm{A}}(\mathrm{t})$. Due to the complexity of the flows in the annular and the non-annular zones and their increasing connectivity to each other, it is only possible to suggest the following "tendency" of the vapor flow in the annular zone.

Under conditions where the mean mass flow rate at the inlet is fixed, the accumulating pulsatile flow kinetic energy being built up (with increasing amplitude of imposed inlet pressure pulsations) in the annular zone vapor control-volume needs to be depleted. This energy build up, for the IF-HA cases, is due to the standing acoustic wave energy that increases with increasing amplitude of inlet pressure pulsations.

At lower amplitudes, this depletion can be carried out by small oscillations of the liquid-vapor interface without any significant change in its mean location relative to the stable annular zone boundary of the vapor control-volume. At higher amplitudes, the asymmetric time-variations of the standing-wave-induced pressure field attempt to deplete the kinetic energy of fluctuations by larger amplitude oscillations of the liquid-vapor interface. 
As discussed in section 2.3.5, when the interfacial wave-troughs come to within tens of micro-meters of the condensing surface, physical phenomena related to even smaller nano-meter length scales become involved. For reasons discussed in section 2.3.5, these phenomena cause the wave-troughs to "stick" near the heat-exchange surface and create rigorous convective eddies adjacent to the interfacial wave-troughs which help keep the interfacial wave peaks from going back toward the N-IF film thickness values. Thus the time-averaged location of the liquid-vapor interface now changes significantly with respect to the steady location associated with the N-IF case such that the time-averaged film thickness is significantly reduced for the IF-HA case relative to the N-IF case.

This change in the time-averaged interface location has to take into account the stability of the interface, greater acoustic wave impedance (at least for the IF-HA cases near Transition Region-1) of the predominantly-liquid region at $x \geq x_{A}(t)$, and relative ease of significantly increasing the kinetic energy efflux out of the liquid-vapor interface.

The above described "tendency" of the annular zone vapor flow with significant standing waves (i.e. IF-HA fully condensing flow cases) will be termed, in this chapter, its fundamental "tendency." For the quasi-steady annular regime IF-HA cases, it appears that the flow mechanisms associated with interfacial waves (and associated heat-flux enhancements) that develop at the annular regime liquid-vapor interface are self-selected to support the above stated fundamental "tendency" of the vapor to deplete its kinetic energy.

Neither the "tendency" nor the detailed mechanisms for the annular regime can be completely established, at the current time. This requires a proper synthesis of more fundamental simulations and/or additional experimental data, which is outside the scope of this chapter.

Such fundamental considerations as they apply to complete condensation will necessarily 
involve a complete computational simulation of a fully condensing IF-HA case (which would include both the annular and the non-annular regimes) which models the vapor compressibility effects while accounting for the following "parabolic" boundary conditions, namely: knowledge of the time-varying inlet mass flow rate, knowledge of steady or time-varying condensing-surface temperatures, and knowledge of the time-varying pressure at a single point in the flow field (e.g. at a point on the inlet cross-section). Knowledge of the time-varying pressure at the test-section exit will not be necessary if such fundamental simulations involve flow situations for which the test-section exit is sufficiently downstream in the all-liquid region. However, simplified simulations may be run for just the annular flow regime if either the time-varying pressure at a location on the exit cross-section of the control-volume or the time-varying flow rate leaving the control-volume is known.

Despite the above limitations, we are able to describe the conjectured detailed mechanisms with regard to the development of the annular zone interfacial waves and its effects. This description is partially supported by the observed experimental data, and parts of the suggested mechanisms are likely to get established or improved with the help of direct computational simulations and additional experimental details.

Consider an inlet mass flow rate oscillation period (of the characteristic time duration $\Delta t_{C} \equiv 1 / f_{P}$ associated with the principal frequency $f_{p}$ ) associated with an IF-HA case and its two constituent time durations (marked as " $\Delta t_{C} / 2$ " in Fig. 3.3). The duration for which the inlet mass flow rates are larger than the mean mass flow rate is termed surge duration. The other duration, for which mass flow rates are smaller than the mean, is termed stall duration. During the surge duration of an IF-HA case, at any fixed location within the annular zone, large-crested waves appear with surging wave-speeds (see Video 3-3 in the accompanying data disc) that are larger than the corresponding N-IF case's wave-speed.

Careful observation shows that the larger wave form during this duration accompanies secondary waves which superpose on the crest of the larger wave form. These secondary 
wave crests rise up toward the end of the stall durations and disappear toward the end of the surge durations. Thus the forward-surging vapor flow experiences the interfacial traction provided by these secondary waves, whereas the slower stalling vapor flow does not.

This behavior is likely related to phase-delay experienced by the instantaneous liquid $\mathrm{X}$-direction velocity vectors relative to the instantaneous vapor $\mathrm{X}$-direction velocity vectors caused by the higher inertia of the liquid phase. If the minimum vapor velocity in the $\mathrm{X}$-direction is lower than the maximum liquid velocity in the $\mathrm{X}$-direction, such a phase shift can cause the direction of the tangential interfacial shear stress component near an interfacial wave peak to switch direction by 180 degrees and back again around the time that the instantaneous vapor velocity value in the $\mathrm{X}$-direction is at a minimum.

This change in direction of the tangential interfacial shear stress could trigger an instability at the interface, causing the secondary waves to rise up just before the vapor velocity increases in the forward direction for the next surge duration of the vapor flow. As the vapor velocity keeps increasing in the forward direction, these secondary waves die out due to the stabilizing effect of the tangential shear stress at the interface, which is now acting in the same direction as the vapor and liquid velocities tangent to the liquidvapor interface. Thus the subsequent stall duration experiences a smoother liquid-vapor interface than the preceding surge duration, which helps the forward acting interfacial shear stresses to reduce less than they increase for the surge duration. Both the primary and secondary waves can clearly be seen in Video 3-3, and the timing of the appearance and disappearance of the secondary waves appears to be consistent with the preceding explanation. This phenomenon (which acts near the wave peaks) acts concurrently with the "sticking" phenomenon (which acts near the wave troughs) discussed in section 2.3.5.

During the stall durations, the wave speeds are closer to zero, but the magnitudes of their differences relative to the N-IF case's wave-speed is smaller than the difference during the surge duration - resulting in an increase in the net forward velocity of the waves. 
Computations are expected to establish that, relative to the N-IF case, the resulting IF-HA wave-structure has a disproportionately higher effective interfacial shear (made up of the $\mathrm{X}$-component of interfacial stress vector which includes pressure and viscous stress contributions at the taller crests) during the surge duration than the amount by which the effective interfacial shear stress is lower during the stall duration.

The contributions of both the primary and secondary wave-structures are expected to be important in the determination of the time-averaged value of the effective shear stress. These wave related asymmetric time-variations with respect to the N-IF case are believed to be facilitated by the presence of harmonics in the pressure field.

As a result of this, as shown in Fig. 3.16, during the surge flow duration the decrease in film thickness $\delta_{40 \mathrm{~cm}}(t)$ with respect to $\left.\bar{\delta}_{40 \mathrm{~cm}}\right|_{\mathrm{N}-\mathrm{IF}}$, is significantly larger than its recovery during the stall duration. This film thickness pattern, obtained from Eq. (3.13), is automatically consistent with the measured time-varying heat-flux pattern $q_{40 \mathrm{~cm}}^{\prime \prime}(t)$ in Fig. 3.13 and the significant enhancements in the mean heat-flux $\overline{\mathrm{q}_{40 \mathrm{~cm}}}$ shown in Fig. 3.6. These trends are also associated with increased annular zone mean interfacial mass-flux values (which are, at $x=40 \mathrm{~cm}$, approximately equal to $\left[\overline{q_{40 \mathrm{~cm}}^{\prime \prime}} / \mathrm{h}_{\mathrm{fg}}\right]$ ) that assist in the aforementioned fundamental "tendency" of the vapor flow to lose its kinetic energy of fluctuations while imparting it to the annular zone liquid condensate.

The existence of Transition Region-2 in Fig. 3.15 marks conditions where heat-flux enhancement slows down with respect to the increasing amplitude of the imposed pulsations. Reasons for this transition region's existence can be several.

First, a representative IF-HA case video (see Video 3-2 in the accompanying data disc) clearly shows that the plug/slug non-annular regimes at $x>x_{A}(t)>x^{*}$ locations oscillate forward and backward with greater amplitude as the principal amplitude of the fluctuating inlet pressure increases. For pressure fluctuation amplitudes at and above 
Transition Region-2, this may be associated with a reduced percentage of acoustic wave energy getting reflected at the highly oscillatory $x=x_{A}(t)$ interface because more of the acoustic pulsation energy is being dissipated in the non-annular flow regimes.

The second reason is conjectured here on the basis of flow observations taken from experimental videos for the IF-HA cases. As the amplitude increases to cross the Transition Region-2 in Fig. 3.15, it is seen that sub-cooled liquid slugs break from the non-annular regime and spray backward (see the end of Video 3-2 and from $3.5-4$ seconds into Video 3-3 around $54-56.5 \mathrm{~cm}$ ), crossing into the annular zone and possibly passing the $\mathrm{X}=\mathrm{X}^{*}$ boundary of interest where the heat-flux is being measured. These cooler liquid droplets may counter the enhancement trend by providing additional liquid-vapor interfaces (which may absorb thermal energy in the vapor domain or acoustic energy associated with vapor pressure/flow pulsations). Based on the limited amount and regions over which this liquid flow reversal and dispersion were observed in the experiments, and the limited temperature-differences involved, this mechanism is expected to be insignificant in influencing the existence and location of Transition Region-2 compared with the first and third potential reasons.

A third potential reason is that there may be a limitation on the rate at which liquid can be transferred through the wave-troughs based on the "sticking" phenomenon described in section 2.3.5, since the troughs themselves are so close to the heat-exchange surface and the interfacial wave speeds are limited by their frequencies and wavelengths. The higher the heat-flux enhancement, the higher the condensation rates, and the more rapidly the condensate must be removed in the downstream direction. This sets a limitation on the further reduction of film thickness for a given set of flow conditions (beyond which even the rigorous convection effects discussed in section 2.3.5 are limited in their ability to support greater liquid velocities in the wave-troughs). This limitation would occur at lower enhancement levels with increased distance along the condensing surface, where more liquid has condensed and must be carried by the liquid film. It is probably the greatest contributor to the existence and location of Transition Region-2 in Fig. 3.15. 
Regardless of the actual mechanisms responsible for limiting the attainable levels of heat-flux enhancement for pulsatile fully condensing flows, it is obvious that beyond a certain level of imposed vapor pressure/flow pulsations the flow finds ways to dissipate the pulsatile kinetic energy to other locations or in other ways than through the annular flow regime liquid-vapor interface and into the heat-exchange surface underneath it.

\subsection{Conclusions}

The significant conclusions from this investigation of the shear-driven completely condensing flows are:

- The reported results are consistent with the fact that, for negligible imposed fluctuation cases involving a steady mean inlet vapor flow rate and steady cooling conditions, the condenser exhibits only one unique "natural" quasi-steady flow and associated pressure-difference. Though comparisons with simulation results are not reported here, these N-IF experimental results are close (within experimental error) to results obtained from the strictly steady simulations for these shear-driven flows.

- It is found that if the amplitudes of inlet pressure fluctuations are above a critical value, the resulting quasi-steady flow's characteristics (in heat-flux and flow morphology) are significantly different from those associated with negligible or subcritical fluctuation amplitudes. Therefore, for various frequencies of interest, there are typically two classes of responses to inlet pressure fluctuations. These are termed supercritical (for the larger amplitudes for which a strong sensitivity to changes in pressure fluctuation amplitude exists) and subcritical (for the smaller amplitudes for which a weak sensitivity to changes in pressure fluctuation amplitudes exists).

- The results reported in sections 3.4.1 - 3.4.5 establish that multiple quasi-steady shear-driven annular flows can be realized for different high pressure-fluctuation amplitudes (supercritical) that are associated with a given quasi-steady mass flow rate and cooling conditions. This leads to multiple and increased levels of mean local heat-flux (heat-flux enhancement). This fact was shown here for two different cooling conditions and over a range of pulsation frequencies. 
- Experimentally, the above-described enhanced heat-flux phenomenon is found to be associated with the presence of harmonics (associated with the dominant frequency of the forward-and-backward moving acoustic pressure pulsations in the vapor phase) in the interior annular zone pressures. The sensitive coupling of these pressure pulsations with the liquid-vapor interface creates a new interfacial wave dynamic behavior that was absent or negligible for low amplitude imposed pulsations. In the annular regime, the resulting interface wave dynamics enhance mean effective interfacial shear stress, reduce mean liquid thickness, and enhance heat-flux.

- The main mechanism hypothesized to be responsible for the reduced mean liquid thickness associated with the above described pulsation-induced interfacial wave dynamics involves interactions of the liquid-vapor interfacial wave troughs with the heat exchange surface where they approach within sufficiently close proximity to enable their interaction with the nm-thick adsorbed layer of liquid on the heat exchange surface and micro-layer convective eddies caused by disjoining pressure effects at each end of a wave trough. This mechanism is explained in more detail in section 2.3.5.

- A sensitivity of pulsatile annular condensing flows to certain types of exit conditions was observed and briefly discussed here. For other conditions, and where the flow becomes all-liquid significantly before the exit of the condenser, the overall behavior of the condenser is governed entirely by time-averaged inlet pressure, timeaveraged inlet flow rate, cooling conditions, and amplitude of imposed pressure or flow fluctuations at the inlet of the condenser with its relevant harmonics.

- Experimental results for pulsatile partially condensing flows are reported in chapter 2. Proper exit condition considerations in the physical design of condensers experiencing partially condensing annular flows, coupled with appropriate operating procedures, can ensure that the heat-flux enhancement reported here can be experimentally realized for these more desirable flows.

Acknowledgment: This work was supported by NASA Grant NNX10AJ59G and NSF Grant CBET-1033591. 


\section{References: chapter 3}

[1] M.T. Kivisalu, P. Gorgitrattanagul, A. Narain, R. Naik, M. Hasan, Sensitivity of shear-driven internal condensing flows to pressure fluctuations and its utilization for heat flux enhancements, International Journal of Heat and Mass Transfer, 56(1-2) (2013) 758-774.

[2] C.J.M. Lasance, R.E. Simons, Advances in high-performance cooling for electronics, Electronics Cooling, 11(4) (2005).

[3] P.E. Phelan, V.A. Chiriac, T.Y.T. Lee, Current and future miniature refrigeration cooling technologies for high power microelectronics, Components and Packaging Technologies, IEEE Transactions on, 25(3) (2002) 356-365.

[4] J.W. Coleman, S. Garimella, Two-phase flow regimes in round, square and rectangular tubes during condensation of refrigerant R134a, International Journal of Refrigeration, 26(1) (2003) 117-128.

[5] H.Y. Wu, P. Cheng, Condensation flow patterns in microchannels, International Journal of Heat and Mass Transfer, 48 (2005) 286-297.

[6] X. Quan, P. Cheng, H. Wu, Transition from annular flow to plug/slug flow in condensation of steam in microchannels, International Journal of Heat and Mass Transfer, 51(3-4) (2008) 707-716.

[7] J.B. Boreyko, C.-H. Chen, Self-propelled jumping drops on superhydrophobic surfaces, Physics of Fluids, 22(9), 091110 (2010).

[8] S. Narayanan, A.G. Fedorov, Y.K. Joshi, On-chip thermal management of hotspots using a perspiration nanopatch, Journal of Micromechanics and Microengineering, 20(7), 075010 (2010). [9] J.H. Goodykoontz, R.G. Dorsch, Local heat-transfer coefficients for condensation of steam in vertical downflow within a 5/8-inch-diameter tube, NASA TN D-3326 (1966).

[10] J.H. Goodykoontz, R.G. Dorsch, Local heat-transfer coefficients and static pressures for condensation of high-velocity steam within a tube, NASA TN D-3953 (1967).

[11] F.G. Carpenter, Heat transfer and pressure drop for condensing pure vapors inside vertical tubes at high vapor velocities, Ph.D. Thesis University of Delaware, 1948.

[12] A. Cavallini, R. Zechchin, High velocity condensation of R-11 vapors inside vertical tubes, Heat Transfer in Refrigeration, (1971) 385-396.

[13] V.P. Carey, Liquid-vapor phase-change phenomena, Hemisphere Publishing Corp., New York, NY 1992.

[14] W. Nusselt, oberflächenkondesation des wasserdampfes, Zeitschrift des Vereines Deutscher Ing. 60 (27), (1916) 541-546.

[15] M. Soliman, J.R. Schuster, P.J. Berenson, A general heat transfer correlation for annular flow condensation, Journal of Heat Transfer, 90(2) (1968) 267-274. 
[16] A. Cavallini, J.R. Smith, R. Zechchin, A dimensionless correlation for heat transfer in forced convection condensation, in: 6th International Heat Transfer Conference, Tokyo, Japan, (1974). [17] M.M. Shah, A general correlation for heat transfer during film condensation inside pipes, International Journal of Heat and Mass Transfer, 22(4) (1979) 547-556.

[18] M.K. Dobson, J.C. Chato, Condensation in smooth horizontal tubes, Journal of Heat Transfer, 120(1) (1998) 193-213.

[19] N.Z. Azer, L.V. Abis, T.B. Swearinger, Local heat transfer coefficients during forced convection condensation inside horizontal tubes, ASHRAE Transactions, (1977) 182-201.

[20] Q. Lu, N.V. Suryanarayana, Condensation of a vapor flowing inside a horizontal rectangular duct, Journal of Heat Transfer, 117(2) (1995) 418-424.

[21] A. Cavallini, G. Censi, D. Del Col, L. Doretti, G.A. Longo, L. Rossetto, C. Zilio, Condensation inside and outside smooth and enhanced tubes - a review of recent research, International Journal of Refrigeration, 26(4) (2003) 373-392.

[22] J.H. Kurita, Experimental investigation of fully condensing downward vapor flows in a vertical tube - unspecified (free) exit condition cases, M.S. Thesis, Michigan Technological University, Houghton, 2007.

[23] S. Mitra, A. Narain, R. Naik, S.D. Kulkarni, A quasi one-dimensional method and results for steady annular/stratified shear and gravity driven condensing flows, International Journal of Heat and Mass Transfer, 54(15-16) (2011) 3761-3776.

[24] J. Kurita, M. Kivisalu, S. Mitra, R. Naik, A. Narain, Experimental results on gravity driven fully condensing flows in vertical tubes, their agreement with theory, and their differences with shear driven flows' boundary-condition sensitivities, International Journal of Heat and Mass Transfer, 54(13-14) (2011) 2932-2951.

[25] K. Seo Young, K. Byung Ha, H. Jae Min, Heat transfer in the thermally developing region of a pulsating channel flow, International Journal of Heat and Mass Transfer, 36(17) (1993) 4257-4266.

[26] A.A. Al-Haddad, N. Al-Binally, Prediction of heat transfer coefficient in pulsating flow, International Journal of Heat and Fluid Flow, 10(2) (1989) 131-133.

[27] J.C. Ludlow, D.J. Kirwan, J.L. Gainer, Heat transfer with pulsating flow, Chemical Engineering Communications, 7(4-5) (1980).

[28] S.E. Hommema, K.A. Temple, J.D. Jones, V.W. Goldschmidt, Heat transfer in condensing, pulsating flows, International Journal of Heat and Mass Transfer, 45(1) (2002) 57-65.

[29] C.W. Roh, M.S. Kim, Enhancement of heat pump performance by pulsation of refrigerant flow using a solenoid-driven control valve, International Journal of Refrigeration, 35(6) (2012) 1547-1557. 
[30] M. Kivisalu, P. Gorgitrattanagul, S. Mitra, R. Naik, A. Narain, Prediction and control of internal condensing flows in the experimental context of their inlet condition sensitivities, Microgravity Science and Technology, 24(3) (2012) 147-155.

[31] M. Kivisalu, N. Gorgitrattanagul, R. Naik, S. Mitra, A. Narain, Shear/Pressure driven internal condensing flows and their sensitivity to inlet pressure fluctuations, in: ASME International Mechanical Engineering Congress \& Expo., IMECE2011, Denver, CO, USA, (2011).

[32] G.L. Wedekind, B.L. Bhatt, An experimental and theoretical investigation into thermally governed transient flow surges in two-phase condensing flow, Journal of Heat Transfer, 99(4) (1977) 561-567.

[33] B.L. Bhatt, G.L. Wedekind, Transient and Frequency Response Characteristics of Two-Phase Condensing Flows: With and without Compressibility, Journal of Heat Transfer, 102(3) (1980) 495-500.

[34] B.L. Bhatt, G.L. Wedekind, A self-sustained oscillatory flow phenomenon in two-phase condensing flow systems, ASME Journal of Heat Transfer, 102(4) (1980) 695-700.

[35] A. Narain, M. Kivisalu, R. Naik, S. Mitra, M. Hasan, Comparative experimental and computational studies for annular condensing and boiling flows in millimeter scale horizontal ducts, in: ASME 2012 Summer Heat Transfer Conference, Rio Grande, Puerto Rico, (2012).

[36] A. Narain, Q. Liang, G. Yu, X. Wang, Direct computational simulations for internal condensing flows and results on attainability/stability of steady solutions, their intrinsic waviness, and their noise sensitivity, Journal of Applied Mechanics, 71(1) (2004) 69-88.

[37] Q. Liang, X. Wang, A. Narain, Effects of gravity, shear and surface tension in internal condensing flows: results from direct computational simulations, Journal of Heat Transfer, 126(5) (2004) 676-686.

[38] S.D. Kulkarni, A. Narain, M.T. Kivisalu, S. Mitra, M.M. Hasan, Condenser performance, Control, and Heat Transfer Enhancement Issues Resulting from Elliptic-Sensitivity of Shear Internal Condensing Flows, International Journal of Transport Phenomena, 13(1) (2012) 15-57.

[39] K.R. Rao, D.N. Kim, J.J. Hwang, Fast fourier transform - algorithms and applications, Springer, 2010.

[40] P. Gorgitrattanagul, The length of the annular regime for condesing flows inisde a horizontal channel - the experimental determination of its values and its trends, M.S. Thesis, Michigan Technological University, Houghton, 2011.

[41] A. Narain, N. Ajotikar, M.T. Kivisalu, A. Rice, M. Zhao, N. Shankar, Obtaining TimeVarying Pulsatile Gas Flow Rates With the Help of Dynamic Pressure-Difference and Other Measurements for an Orifice-Plate Meter, Journal of Fluids Engineering, 135(4), 041101 (2013) $1-19$. 
[42] R.D. Knight, Physics for scientists and engineers: a strategic approach with modern physics and mastering physics Second ed., Addison Wesley, 2007.

[43] R. Feynman, Lectures in Physics, Addison-Wesley Pub. Co., Addison, 1969. 



\section{Summary and suggestions for future work}

\subsection{Summary}

Chapter 2 reported the response of condensing and boiling flows, in which the flow was annular throughout the length of the test-section, to imposed pressure / flow rate pulsations of various amplitudes at a few different frequencies. Chapter 3 reported the response of completely condensing flows to imposed pressure / flow rate pulsations of various amplitudes at a few different frequencies for two distinct condensing-surface cooling conditions.

For all experiments reported in chapters $2-3$, moderate to extreme enhancement in heat-flux at a location within the condenser or boiler (test-section) was observed for sufficiently large amplitudes of the imposed pressure / flow rate pulsations. Increases in the rate of fluid condensed or boiled as a result of the imposed pressure / flow rate pulsations were also observed, but they were minor compared with the directly measured time-averaged heat-flux increases (enhancement) at the selected measurement location within the test-section due to the imposed pressure / flow rate pulsations.

For experimental boiling cases reported in chapter 2, liquid flow rate pulsations significantly affected time-varying values of absolute pressures and pressure-differences within the boiler, but they did not significantly influence their time-averaged values or the heat-flux measured at a location $40 \mathrm{~cm}$ downstream of the test-section inlet unless vapor pressure / flow rate pulsations were added at the same frequency. These liquid pulsations were inadvertent, as they were caused by the rollers of the peristaltic pump $P_{1}$ (see Fig. 2.5) used to supply liquid to the test-section. Since their frequency was dependent on the pumping rate, this severely limited the range of frequencies which could be investigated in which the frequency of the imposed vapor pulsations was matched to the frequency of the liquid pulsations. It was found through experiments not reported here that if these frequencies are not compatible, there is much less, if any, heat-flux enhancement measured at the $x=40 \mathrm{~cm}$ location in the test-section. 


\subsection{Open issues and future work}

\subsubsection{Opportunities for new experimental work}

Open issues related to the condensing and boiling flows presented in chapters $2-3$ are:

- More work needs to be done to understand the interactions between acoustics, vapor core turbulence (for very high flow rates), interfacial shear stress, and surface effects (involving the interaction of nano-, micro-, and macro-scale phenomena within the interfacial wave-troughs) for pulsation-enhanced shear/pressure-driven innovative flow condensation and flow boiling.

- More work needs to be done to characterize desirable combinations of pulsation frequency, exit condition arrangement, and flow control strategies, which will lead to effective and reliable performance of innovative annular flow condensers and boilers throughout their length by promoting beneficial interaction of the underlying physical mechanisms.

- More work needs to be done to characterize the interaction between liquid and vapor flow rate / pressure pulsations at the inlet of a flow boiler with separate liquid and vapor injection, as in experimental boiling cases reported in chapter 2.

- More work needs to be done to understand the role of non-annular flow regimes in the attainment of heat-flux enhancement in the annular region of completely condensing flows. Some suggestions on the role of the non-annular flow regimes have been provided in sections 3.4.3.2 and 3.4.5 based on limited experimental observation, but these hypotheses need to be tested experimentally.

- Investigation of the re-circulating vapor flow rates and their time-variations as feedback control loops on all-annular condensing and boiling flows, and analysis of their frequency response characteristics, would enable the development of more sophisticated flow control strategies for condensing and boiling flows in the innovative configuration of Figs. 2.1b and 2.2b. Such work is recommended for future optimization of, and development of diagnostic tools for, system designs which use re-circulating vapor flows in their boilers and condensers. 


\subsubsection{Equipment issues affecting data reported here}

Experimental equipment issues affecting the experimental results in chapters $2-3$ which should be rectified for future experiments are:

- $\quad$ Surface-temperature controls using the TECs of Fig. 2.3c (reversed TECs of Fig. 2.5) need to be improved when used with boiling flows. Limitations of the existing temperature measurements and surface-temperature controls with regard to boiling flows are mentioned in section 2.3.4.

- Better heat-exchange surface temperature measurements are needed to allow a proper characterization of the condensing or boiling surface cooling/heating condition. For both boiling and condensing flows investigated in chapters 2 and 3 , there are no measurements of surface temperature near the center of the heat removal/addition locations (above the TECs and heat sinks of Figs. $2.3 \mathrm{c}-2.5$ and Figs. $3.1 b-3.2$ ). Instead, all the surface-temperature measurements reported in chapters $2-3$ were taken in between heat removal/addition locations, as shown by the thermocouple locations in Figs. 2.3c and 3.1b. This means that reported spatially-averaged condensing surface temperatures are higher than their actual values, and reported spatially-averaged boiling surface temperatures are lower than their actual values. Accordingly, values of spatially-averaged $\Delta \mathrm{T}_{0-40}$ and $\Delta \mathrm{T}_{0-88.5}$ reported in Table 2.1 and $\Delta \mathrm{T}$ reported in Table 2.3 (and in Figs. 2.8 and 2.11) are lower than their actual values for both condensing and boiling flows. This also applies to $\bar{T}_{\mathrm{w} \mid \text { Ref }}$ values reported in Table 3.1 - they are higher than their actual values, closer to the value of $T_{\text {sat }}$. The magnitude of this discrepancy in spatially-averaged heat-exchange-surface temperatures needs to be characterized, and new experiments should be performed with appropriately distributed temperature measurements.

- Correct cold junction compensation should be used for future experiments. All the thermocouple measurements reported in chapter 2 needed to be corrected after data collection because of errors in applying cold junction compensation. 


\subsubsection{Relevant suggested, planned, and ongoing research}

The works reported in chapters $2-3$ facilitate and guide the ongoing research on:

- Pulsatile flow experiments with a shorter length test-section. The pulsations and exit conditions are to be arranged to have large amplitude standing waves on a thin liquid boiling or condensing film over the entire test-section length. The goal is to attain high levels of overall heat load enhancement well above the mechanical energy supplied by the pulsations. This will be measured by an increased mass flow rate of liquid boiled or condensed for the same saturation temperature and equivalent heatexchange surface-temperature profiles.

- Pulsatile flow boiling experiments with controlled imposition of liquid flow rate pulsations using a liquid pulsator $\left(P_{L}\right.$ in Fig. 2.5), coupled with suitable damping of pulsations arising from the peristaltic liquid pump ( $P_{1}$ in Fig. 2.5), to ensure that a large range of vapor and liquid pulsation frequencies can be investigated.

- Pulsatile flow experiments with additional temperature measurements to properly characterize the spatially-averaged heat-exchange-surface temperatures and allow estimation of the order of magnitude of the discrepancy - in $\Delta T_{0-40}$ and $\Delta T_{0-88.5}$ values reported in Tables 2.1, $\Delta \mathrm{T}$ values reported in Table 2.3, and $\overline{\mathrm{T}}_{\mathrm{w} \mid \mathrm{Ref}}$ values reported in Table 3.1 - due to the use of non-characteristic temperature measurement locations.

- Pulsatile flow simulations to support the experimental findings in chapters $2-3$ and ongoing experiments. This will extend the current simulation ability which has been demonstrated for "non-pulsatile" flow cases.

- Pulsatile flow simulations to capture some of the physical mechanisms hypothesized in chapters $2-3$ (such as the effects of surface tension and the "adsorbed layer" on the wave-troughs, development of standing acoustic waves in the test-section and their influences on the liquid-vapor interface, etc.).

- Pulsatile flow simulations to help answer the open questions regarding the relative importance of various secondary effects (such as transient vapor compressibility, vapor core turbulence, etc.) on the heat-flux enhancement phenomenon. 


\section{Conclusions}

The results in chapters $2-3$ lead to the following conclusions.

\subsection{All-annular flow in condensers and boilers}

- Vapor re-circulation at controlled flow rates is shown in chapter 2 to be effective at maintaining stable annular flow over the entire length of a horizontal condenser or boiler. Selection of the vapor re-circulation flow rate was found to enable precise control of the range of vapor quality experienced in the condenser or boiler without excessive increase in the pressure-difference across the device.

- The use of liquid re-circulation in addition to the vapor re-circulation is recommended in section 2.1 (see also sections 2.2.1 and 2.2.2.2) for boilers operating through non-temperature controlled heating methods to avoid critical heat-flux and dry-out conditions.

- If the vapor and, if used, liquid re-circulation flow loops are considered to be a part of the condenser or boiler, that device may be configured to interact with the rest of its flow system in a manner equivalent to a device utilizing complete condensation or complete boiling. This is demonstrated in Fig. 2.2b for a flow boiler with recirculating vapor and liquid loops, in which the net fluid flow into the device is pure liquid, and the net fluid flow out of the device is pure vapor.

- For a flow boiler operating in the all-annular configuration of Fig. 2.2b, a separator plate (Fig. 2.3b) was successfully used to direct the injected liquid over the heat-exchange surface. This is apparent in Videos 2-3 - 2-4.

\subsection{Pulsation-induced heat-flux enhancement}

Externally imposed fluctuations in vapor pressure/flow rate are effective in reducing fluid to heat-exchange surface thermal resistance for condensers and boilers operating in the all-annular innovative flow configuration of Figs. $2.1 \mathrm{~b}$ and $2.2 \mathrm{~b}$, and for traditional fully condensing flows as in Figs. 3.1a and 3.2. 


\subsubsection{Heat-flux enhancement measured experimentally}

- Heat-flux enhancements were observed to approach $900 \%$ relative to comparable flows without imposed fluctuations for all-annular condensing flows at a representative measurement location under certain operating conditions.

- Heat-flux enhancements were observed to approach $200 \%$ relative to comparable flows without imposed fluctuations for all-annular boiling flows at a representative measurement location under certain operating conditions.

- Heat-flux enhancements were observed to approach $500 \%$ relative to comparable flows without imposed fluctuations for completely condensing flows at a representative measurement location under certain operating conditions.

- Condensing flows in the innovative (all-annular) configuration were observed to show a marked frequency-dependence which was not significant for traditional completely condensing flows.

\subsubsection{Mechanisms behind observed heat-flux enhancement}

- The physical mechanism behind the observed enhanced heat-flux phenomenon is hypothesized in section 2.3.5 to arise from solid-liquid-vapor effects on the liquid-vapor interfacial wave-troughs when they approach to within tens of $\mu \mathrm{m}$ of the heat exchange surface under the influence of suitable local vapor pressure/flow pulsations. As a result, through the interaction of physical phenomena at 3 length scales and 2 time scales described in section 2.3.5, there is an asymmetrical tendency of the liquid-vapor interface to remain near the heat-exchange surface.

- The thermal resistance of the heat flow through the liquid film is approximately proportional to the film thickness when conduction heat transfer dominates, as is known to be the case for these annular flows when the film is sufficiently thin. Therefore, reduction in the time-averaged distance of the liquid-vapor interface from the heat-exchange surface (film thickness) at a particular location within the condenser or boiler results in increased time-averaged heat-flux at that location.

- Observations described in sections 3.4.4 - 3.4.5 suggest that the observed heat-flux phenomenon is influenced by the presence of harmonics (fluctuations at 
multiples of the imposed pulsation frequency) in the interior annular zone pressures. These harmonics are likely caused by approximately "standing" forward-and-backward moving acoustic pressure pulsations in the vapor phase.

- The sensitive coupling of the pressure pulsations from the vapor phase (with their harmonics) with the liquid-vapor interface in the annular flow regime provides the driving force for the high amplitude waves of the liquid-vapor interface needed to allow the previously mentioned physical mechanism to take effect. It may also enable secondary physical phenomena (such as wave-texturing, mentioned in sections 2.3.5 and 3.4.5) which contribute to heat-flux enhancement.

- The reduced frequency-dependence of the pulsation-induced heat flux enhancement phenomenon observed for traditional fully condensing flows is hypothesized to be caused by the "active" nature (due to compressibility of confined vapor slugs/bubbles) of the plug/slug and bubbly flow regimes. This spring-like effect provides conditions at the exit of the annular flow regime suitable for encouraging large amplitude forward-and-backward moving acoustic waves within the vapor domain of the condenser over a wide range of frequencies.

\subsubsection{Heat-flux enhancement limitations and recommendations}

- For condensing and boiling flows in the innovative configuration of Figs. $2.1 b-2.2 b$, it is suggested that a vibrating plate or similar device near the exit of the condenser or boiler (see Fig. 2.2b) be used to create "active" exit conditions which would improve the heat-flux enhancement and extend its usefulness over a wider range of frequencies.

- For boiling flows in the innovative configuration of Fig. 2.2b, it is found that inadvertent pulsations in the liquid supplied to the device have a profound influence on the vapor pressure / flow rate pulsation frequencies which will lead to significant heat-flux enhancement - specifically, the vapor pulsation frequency should match the liquid pulsation frequency to achieve maximum enhancement.

- For all-annular condensing flows in the innovative configuration of Fig. 2.1b, consideration of condensing-surface temperatures in Figs. 2.12 - 2.13 and 
heat-exchange surface thermal resistance suggests that heat-flux enhancement occurred over certain regions along the length of the condenser, separated by regions of little or no enhancement. This is consistent with the suggestion from section 5.2.2 that "standing" acoustic waves (with pressure nodes and antinodes at defined locations within the flow condenser) are a contributing factor to the observed heat-flux enhancement phenomenon.

- For completely condensing flows in the configuration of Fig. 3.1a under the "passive" cooling condition defined in section 3.3.3, consideration of condensing-surface temperatures in Fig. 3.7 and heat-exchange surface thermal resistance suggests that heat-flux enhancement occurred over the entire length of the condenser. This may be a result of the increased significance of harmonics and other pressure pulsations in the vapor phase enabled by the "active" non-annular flow regimes as previously mentioned.

- Based on chapters 2-4 and the preceding conclusions, it is expected that substantial heat-flux enhancement will be observed over most or all of the heat-exchange surface for condensers and boilers operating in the innovative configuration of Figs. 2.1b and 2.2b through effective selection of one or more imposed pulsation frequencies, implementation of an active flow element (tuned to the desired pulsation frequency or frequencies) near the device exit, and use of appropriate flow control strategies. 